\title{
Inferência e diagnóstico em modelos assimétricos
}

\author{
Clécio da Silva Ferreira
}

TESE APRESENTADA

$\mathrm{AO}$

Instituto de Matemática e Estatística

DA

Universidade DE SÃo PAUlo

PARA

OBTENÇÃO DO TÍTULO

$\mathrm{DE}$

Doutor EM CIÊNCIAS

\author{
Área de Concentração: Estatística \\ Orientador: Prof. Dr. Heleno Bolfarine
}

São Paulo, março de 2008 


\section{Inferência e diagnóstico em modelos assimétricos}

Este exemplar corresponde à redação final da tese devidamente corrigida e defendida por Clécio da Silva Ferreira e aprovada pela Comissão Julgadora.

Banca Examinadora:

- Prof. Dr. Heleno Bolfarine (orientador) - IME-USP.

- Prof. Dr. Reinaldo Boris Arellano-Valle - PUC-Chile

- Prof. Dr. Gilberto Alvarenga Paula - IME-USP.

- Prof. Dr. Mário de Castro Andrade Filho - ICMC-USP

- Prof. Dr. Francisco Louzada-Neto - UFSCar. 
"All models are wrong, but some models are useful." George Box

"Plante seu jardim e decore sua alma, ao invés de esperar que alguém lhe traga flores."

William Shakespeare

"O valor das coisas não está no tempo que elas duram, mas na intensidade com que acontecem. Por isso, existem momentos inesquecíveis, coisas inexplicáveis e pessoas incomparáveis." 
Dedico este trabalho:

A toda minha família, em especial à minha filha Letícia, que trouxe muito amor, luz e paz na minha vida. 


\section{Agradecimentos}

À minha família, esposa, filha, mãe, pai (in memorian), irmãos(ãs) e sobrinhos(as), pelo apoio e incentivo durante todos os anos de estudos.

A meu orientador, Prof. Dr. Heleno Bolfarine, pela confiança, apoio e direcionamento durante a elaboração deste trabalho.

Ao Prof. Dr. Víctor Hugo Lachos Dávila que, mesmo não sendo oficialmente meu coorientador, foi de fundamental importância para o sucesso deste trabalho. Sua experiência em pesquisa na área de modelos lineares e computação estatística contribuiu significativamente em cada capítulo desta tese.

Aos membros da banca examinadora, Prof. Dr. Reinaldo Boris Arellano-Valle (Puc-Chile), Prof. Dr. Gilberto Alvarenga Paula (IME-USP), Prof. Dr. Mário de Castro (ICMC-USP) e Prof. Dr. Francisco Louzada-Neto (UFSCar), pela leitura, correções e sugestões da tese.

Aos professores do Departamento de Estatística do IME-USP, pelos ensinamentos concedidos.

Aos funcionários do IME-USP, pela presteza e dedicação no atendimento.

Ao Centro de Estudos de Opinião Pública (CESOP-UNICAMP), onde trabalho desde 2002, inicialmente como bolsista e membro da carreira de Pesquisador (a partir de 2004), pelo apoio e tempo concedidos durante as atividades do curso.

A todos os meus amigos e amigas com quem tive a honra de conviver: na minha querida cidade de UBÁ-MG, onde nasci e cresci; na Universidade Federal de Viçosa, onde fiz a graduação em Matemática; na Universidade Estadual de Campinas, onde fiz meu mestrado em Estatística; na Universidade de São Paulo (doutorado) e no CESOP-UNICAMP, local de trabalho.

A todos, o meu muito obrigado.

Clécio da Silva Ferreira 


\section{Resumo}

Este trabalho apresenta um estudo de inferência e diagnósticos em modelos assimétricos. A análise de influência é baseada na metodologia para modelos com dados incompletos, que é relacionada ao algoritmo EM (Zhu e Lee, 2001). Além dos modelos de regressão Normal Assimétrico (Azzalini, 1999) e t-Normal Assimétrico (Gómez, Venegas e Bolfarine, 2007) existentes, são desenvolvidas duas novas classes de modelos, denominados modelos de misturas de escalas normais assimétricos (englobando as distribuições Normal, t-Normal, Slash, Normal-Contaminada e Exponencial-potência Assimétricas) e modelos lineares mistos robustos assimétricos, utilizando distribuições de misturas de escalas normais assimétricas para o efeito aleatório e distribuições de misturas de escalas para o erro aleatório. Para o modelo misto, a matriz de informação de Fisher observada é calculada utilizando a aproximação de Louis (1982) para dados incompletos. Para todos os modelos, algoritmos tipo EM são desenvolvidos de forma a fornecer uma solução numérica para os parâmetros dos modelos de regressão. Para cada modelo de regressão, medidas de bondade de ajuste são realizadas via inspeção visual do gráfico de envelope simulado. Para os modelos de misturas de escalas normais assimétricos, um estudo de robustez do algoritmo EM proposto é desenvolvido, determinando a eficácia dos estimadores apresentados. Aplicações dos modelos estudados são realizadas para os conjuntos de dados do Australian Institute of Sports (AIS), para o conjunto de dados sobre qualidade de vida de pacientes (mulheres) com câncer de mama, em um estudo realizado pelo Centro de Atenção Integral à Saúde da Mulher (CAISM) em conjunto com a Faculdade de Ciências Médicas, da Universidade Estadual de Campinas e para o conjunto de dados de colesterol de Framingham.

Palavras-chave: Distribuições Normais Assimétricas, Distribuições de Misturas de Escala Normal, Modelos Mistos, Algoritmo EM. 


\section{Abstract}

This work presents a study of inference and diagnostic in asymmetric models. The influence analysis is based in the methodology for models with incomplete data, that is related to the algorithm EM (Zhu and Lee, 2001). Beyond of the existing asymmetric normal (Azzalini, 1999) and t-Normal asymmetric (Gómez, Venegas and Bolfarine, 2007) regression models, are developed two new classes of models, namely asymmetric normal scale mixture models (embodying the asymmetric Normal, t-Normal, Slash, ContaminatedNormal and Power-Exponential distributions) and asymmetric robust linear mixed models, utilizing asymmetric normal scale mixture distributions for the random effect and normal scale mixture distributions for the random error. For the mixed model, the observed Fisher information matrix is calculated using the Louis' (1982) approach for incomplete data. For all models, EM algorithms are developed, that provide a numeric solution for the parameters of the regression models. For each regression model, measures of goodness of fit are realized through visual inspection of the graphic of simulated envelope. For the asymmetric normal scale mixture models, a study of robustness of the proposed EM algorithm is developed to determine the efficacy of the presented estimators. Applications of the studied models are made for the data set of the Australian Institute of Sports (AIS), for the data set about quality of life of patients (women) with breast cancer, in a study made by Centro de Atenção Integral à Saúde da Mulher (CAISM) in conjoint with the Medical Sciences Faculty, of the Campinas State's University and for the data set of Framingham's cholesterol study .

Keywords: Asymmetric Normal Distributions, Scale Mixtures of Normal Distributions, Mixed Models, EM Algorithm. 


\section{Sumário}

Lista de Figuras

p. vii

Lista de Tabelas

p. xii

1 Introducão

p. 1

2 Modelo Normal Assimétrico

p. 16

2.1 Distribuição Normal Assimétrica Padrão . . . . . . . . . . . . . . . . p. 16

2.2 Distribuição Normal Assimétrica de Locação-Escala . . . . . . . . . . . . . p. 19

2.3 Estimação de Máxima Verossimilhança via Algoritmo EM . . . . . . . . p. 21

2.4 Matriz de Informação de Fisher Observada . . . . . . . . . . . . . . . . p. 23

2.5 Diagnóstico no Modelo Normal Assimétrico . . . . . . . . . . . . . . p. 25

2.5.1 A matriz hessiana . . . . . . . . . . . . . p. 26

2.5.2 Perturbação de ponderação de casos . . . . . . . . . . . . . . . p. 26

2.5.3 Perturbação do parâmetro de escala . . . . . . . . . . . . . . p. 27

2.5.4 Perturbação do parâmetro de assimetria $\ldots \ldots \ldots$. . . . . . p. 27

2.5.5 Perturbação em uma variável explicativa . . . . . . . . . . . . . p. 28

2.5.6 Perturbação na variável resposta . . . . . . . . . . . . . . . p. 28

2.5.7 Alavancagem generalizada . . . . . . . . . . . . . . . p. 29

2.6 Estudo de Dados Artificiais . . . . . . . . . . . . . . . . . . . . p. 29

2.7 Aplicação . . . . . . . . . . . . . . . . . . . . . . . . . . . . . . p. 32 
2.8 Falta de Ajuste no Modelo Normal Assimétrico $\ldots \ldots$. . . . . . . . . p.37

3 Modelo t-Normal Assimétrico p. 38

3.1 Introdução . . . . . . . . . . . . . . . . . . . . . . . . . p. 38

3.2 Modelo de Regressão t-Normal Assimétrico e Algoritmo EM . . . . . . . p. 40

3.3 Matriz de Informação Observada . . . . . . . . . . . . . . . . p. 43

3.4 Diagnóstico no Modelo t-Normal Assimétrico . . . . . . . . . . . . . . . p. 44

3.4 .1 A matriz hessiana . . . . . . . . . . . . . . . p. 44

3.4 .2 Perturbação de ponderação de casos . . . . . . . . . . . . . . p. 45

3.4.3 Perturbação do parâmetro de escala . . . . . . . . . . . . . . . p. 46

3.4.4 Perturbação do parâmetro de assimetria . . . . . . . . . . . p. 46

3.4.5 Perturbação na variável explicativa . . . . . . . . . . . . p. 47

3.4.6 Perturbação na variável resposta . . . . . . . . . . . . . p. p. 47

3.5 Estudo de Dados Artificiais . . . . . . . . . . . . . . p. 48

3.6 Aplicação . . . . . . . . . . . . . . . . . . . p. 54

4 Modelo de Misturas de Escala Normal Assimétrico p. 60

4.1 Introdução . . . . . . . . . . . . . . . . . . . . . . p. 60

4.2 Distribuições de Misturas de Escala Normal . . . . . . . . . . . . . . p. 61

4.2.1 Exemplos de distribuições MEN . . . . . . . . . . . . . . . p. 62

4.3 Distribuições de Misturas de Escala Normal Assimétricas . . . . . . . . . . p. 64

4.3.1 Exemplos de distribuições MENA . . . . . . . . . . . . . . p. 68

4.4 Modelos de Regressão MENA e o Algoritmo EM . . . . . . . . . . . . . . p. 71

4.4.1 Distribuições condicionais para o algoritmo EM . . . . . . . . p. 73

4.5 Matriz de Informação de Fisher Observada . . . . . . . . . . . . . . p. 75 
4.6 Estudo de Convergência do Algoritmo EM . . . . . . . . . . . . . p. p.

4.7 Análise de Diagnóstico . . . . . . . . . . . . . . . . . . . p. 81

4.7 .1 A matriz hessiana . . . . . . . . . . . . . . . p. 82

4.7.2 Perturbação de ponderação de casos . . . . . . . . . . . . . p. p 82

4.7.3 Perturbação do parâmetro de escala . . . . . . . . . . . . . p. 83

4.7.4 Perturbação do parâmetro de assimetria . . . . . . . . . . . p. 83

4.7.5 Perturbação em uma variável explicativa . . . . . . . . . . . . . p. 84

4.7.6 Perturbação na variável resposta . . . . . . . . . . . . . . p. 85

4.7 .7 Alavancagem generalizada . . . . . . . . . . . . p. 85

4.8 Estudo de Simulação . . . . . . . . . . . . . . . . . . . . . p. 86

4.9 Aplicação . . . . . . . . . . . . . . . . . . . . . p. 88

5 Modelo Linear Misto Assimétrico p. 94

5.1 Introdução . . . . . . . . . . . . . . . . . p. . . . . . . . .

5.2 Modelo Linear Misto de Misturas de Escala Normal Assimétrico . . . . . . p. 95

5.3 Algoritmo EM . . . . . . . . . . . . . . . . . p. 100

5.3.1 Distribuições condicionais para o algoritmo EM . . . . . . . . . p. 101

5.3.2 O modelo misto normal assimétrico . . . . . . . . . . . . p. 104

5.4 Matriz de Informação Observada . . . . . . . . . . . . . p. 106

5.5 Diagnóstico no Modelo Linear Misto Robusto . . . . . . . . . . . . . p. 111

5.5.1 A matriz hessiana . . . . . . . . . . . . . . p. 111

5.5.2 Perturbação de ponderação de casos . . . . . . . . . . . . . . . . . . p. 112

5.5.3 Perturbação do parâmetro de escala do erro . . . . . . . . . . . . p.112

5.5.4 Perturbação do parâmetro de escala do efeito aleatório . . . . . . . p.113

5.5.5 Perturbação do parâmetro de assimetria . . . . . . . . . . . . p. 114 
5.5.6 Perturbação em uma variável explicativa . . . . . . . . . . . . . p. 114

5.5.7 Perturbação na variável resposta . . . . . . . . . . . . . p. 115

5.6 Estudo de Dados Artificiais . . . . . . . . . . . . . . p. 116

5.7 Aplicação . . . . . . . . . . . . . . . . . . . . . . . p. 118

6 Considerações Finais $\quad$ p. 122

6.1 Conclusões . . . . . . . . . . . . . . . . . . . . . . p. 122

6.2 Perspectivas para Pesquisas Futuras . . . . . . . . . . . . . . p. 123

Apêndice A - Modelo Normal Assimétrico $\quad$ p. 126

A.1 Matriz de Informação de Fisher Esperada . . . . . . . . . . . . p. 126

Apêndice B - Modelo t-Normal Assimétrico $\quad$ p. 129

B.1 Gráficos de Diagnóstico para os Dados Simulados . . . . . . . . . . . p. 130

Apêndice C - Modelo de Misturas de Escala Normal Assimétrico p. 133

C.1 Função Densidade de Probabilidade de Distribuições de Misturas de Escala Normal Assimétricas . . . . . . . . . . . . . . . . . . . . p. 133

C.2 Gráficos de Diagnósticos . . . . . . . . . . . . . . . . p. 134

$\begin{array}{ll}\text { Referências } & \text { p. } 139\end{array}$

Ferreira, C. S. 


\section{Lista de Figuras}

2.1 Função densidade $N A(\lambda)$, para diferentes valores de $\lambda . \ldots \ldots$. . . . . . 17

2.2 Modelo de regressão com dados simulados. (a) Gráfico de dispersão, juntamente com as retas do modelo normal assimétrico simulado e ajustado e pontos influentes e (b) gráfico de envelope simulado. . . . . . . . . . . p. p.30

2.3 Diagnóstico para o modelo de regressão com dados simulados. Linhas horizontais são os pontos de corte $\left(c^{*}=3\right.$ ). (a) Perturbação de ponderação de casos, (b) perturbação na escala, (c) perturbação na variável resposta, (d) perturbação na variável explicativa, (e) perturbação em $\lambda$ e (f) alavanca. p.31

2.4 Influência global para o modelo de regressão com dados simulados. (a)

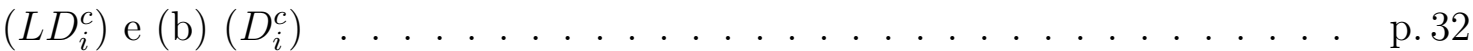

2.5 Ajuste do modelo normal assimétrico para o conjunto de dados sobre qualidade de vida. (a) histograma do resíduo do modelo e (b) gráfico de envelope simulado.

2.6 Ajuste do modelo de regressão múltipla (normal) para o conjunto de dados sobre qualidade de vida. (a) histograma do resíduo do modelo e (b) gráfico de envelope simulado. . . . . . . . . . . . . . . . . . . . p. 34

2.7 Diagnóstico para o conjunto de dados de qualidade de vida. (a) influência global $\left(L D_{i}^{c}\right)$, (b) influência global $\left(D_{i}^{c}\right)$ e (c) gráfico de dispersão $i m c \times p c s$, por tontura, com pontos influentes. . . . . . . . . . . . . . p. 35

2.8 Diagnóstico para o conjunto de dados de qualidade de vida. Linhas horizontais são os pontos de corte $\left(c^{*}=3\right)$. (a) Perturbação de ponderação de casos, (b) perturbação na escala, (c) perturbação na variável resposta, (d) perturbação na variável explicativa, (e) perturbação em $\lambda$ e (f) alavanca. p.36 
2.9 Dados simulados da $N A(10,1,5), n=200$, com pontos perturbados na cauda pesada da amostra. (a) Histograma e (b) envelope simulado. . . . . p. 37

3.1 Densidades $T N A_{3}(\lambda) \ldots \ldots \ldots \ldots \ldots \ldots \ldots$

3.2 Envelope simulado para os dados simulados de uma população $N A(10,1,5)$, $n=200$, com pontos perturbados na cauda pesada da distribuição. . . . . p. 43

3.3 Gráficos de envelope simulado para o conjunto de dados simulados. (a) Modelo normal simétrico e (b) modelo normal assimétrico. . . . . . . . . . p. 49

3.4 Modelo de regressão t-normal assimétrico com dados simulados. (a) Gráfico de dispersão, juntamente com as retas do modelo t-normal assimétrico simulado e ajustado e pontos influentes e (b) gráfico de envelope simulado. . . p.50

3.5 Gráficos de envelopes: (a) Perturbação $y(57)=10$ e (b) perturbação $y(33)=$

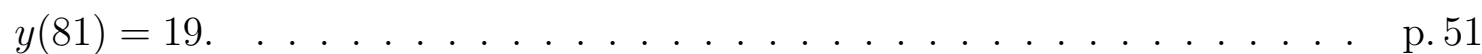

3.6 Gráficos de diagnóstico para o modelo de regressão TNA simulado, sem perturbações. Linhas horizontais são os pontos de corte $\left(c^{*}=3\right)$ : (a) Perturbação de ponderação de casos, (b) perturbação no parâmetro de escala, (c) perturbação na variável resposta, (d) perturbação na variável explicativa e (e) pertubação no parâmetro de assimetria. . . . . . . . . . . . . . p.52

3.7 Influência global para o modelo de regressão TNA simulado, sem perturbações: (a) $L D_{i}^{c}$ e (b) $D_{i}^{c} \ldots \ldots \ldots \ldots \ldots \ldots$. . . . . . . . . . . . . . . . . . . . . .

3.8 Gráficos de envelope simulado para o conjunto de dados AIS: (a) modelo normal simétrico e (b) modelo normal assimétrico. . . . . . . . . . . p.56

3.9 Ajuste de modelo de regressão t-normal assimétrico para os dados AIS. (a) Gráfico de dispersão, juntamente com a reta ajustada e pontos influentes e (b) gráfico de envelope simulado. . . . . . . . . . . . . . . . . p.56

3.10 Conjunto de dados AIS. (a) Afastamento pela verossimilhança $L D_{i}^{c}$ e (b)

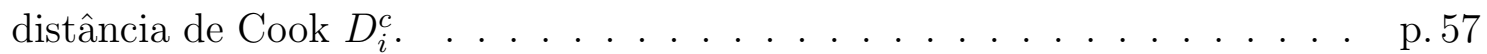


3.11 Gráficos de diagnóstico para o conjunto de dados AIS: (a) Perturbação de ponderação de casos, (b) perturbação no parâmetro de escala, (c) perturbação na variável explicativa e (d) perturbação na variável resposta. Linhas horizontais são os pontos de corte $\left(c^{*}=3\right) \ldots \ldots \ldots$. . . . . . . . .

4.1 Funções de densidade das distribuições normal $N A(3)$, t-normal generalizada $G t A(3 ; 2,2)$, slash $S L A(3 ; 0.5)$, normal contaminada $N C A(3 ; 0.9,0.1)$ e exponencial potência $E P A(3 ; 0.5)$ assimétricas. . . . . . . . . . . p.70

4.2 Boxplots das médias amostrais (painel à esquerda) e medianas amostrais (painel à direita) baseadas em 500 amostras de tamanho $\mathrm{n}=100$ das cinco distribuições padrão $\left(\mu=0\right.$ e $\left.\sigma^{2}=1\right): N A(3) ; G t A(3 ; 3,3) ; N C A(3 ; 0.5,0.5)$; $S L A(3 ; 1)$, e $\operatorname{EPA}(3 ; 0.5)$. As respectivas médias foram ajustadas para zero. p. 87

4.3 Conjunto de dados AIS. (a) Histograma do resíduo do modelo normal contaminado ajustado e (b) gráfico de dispersão bfat $\times s s f$, juntamente com reta ajustada e pontos influentes. . . . . . . . . . . . . . p. 89

4.4 Conjunto de dados AIS. Gráficos Q-Q e envelope simulado: (a) modelo normal assimétrico, (b) modelo t-normal assimétrico, (c) modelo normal contaminado assimétrico, (d) modelo slash assimétrico, (e) modelo exponencial potência assimétrico e (f) função log-verossimilhança perfilada para o modelo normal contaminado assimétrico. . . . . . . . . . . . . p.91

4.5 Diagnóstico de influência para os dados AIS, sob o modelo normal contaminado assimétrico. (a) ponderação de casos, (b) perturbação na escala, (c) perturbação na variável resposta, (d) perturbação na variável explicativa, (e) perturbação no parâmetro de assimetria e (f) alavanca. Linhas horizontais são os pontos de corte $\left(c^{*}=3\right) \ldots \ldots \ldots$. . . . . . . . . . 92

4.6 Influência global para o conjunto de dados AIS, sob o modelo normal contaminado. (a) $L D i_{c}$ e (b) $D i_{c} \ldots \ldots \ldots \ldots \ldots$. . . . . . . . . . . . . . . . . . . . 
5.1 Diagnóstico no modelo de regressão misto t-normal assimétrico com dados simulados. (a) Ponderação de casos, (b) perturbação no parâmetro de assimetria, (c) perturbação no parâmetro de escala do erro, (d) perturbação no parâmetro de escala do efeito aleatório, (e) perturbação na variável resposta e (f) perturbação na variável explicativa. . . . . . . . . . . . p. 117

5.2 Histograma da variável colesterol (dados de Framingham). . . . . . . . . p. 118

5.3 Análise de diagnóstico no modelo de regressão misto normal contaminado assimétrico para os dados de colesterol (pontos representam indivíduos). (a) Ponderação de casos, (b) perturbação no parâmetro de assimetria, (c) perturbação no parâmetro de escala do erro, (d) perturbação no parâmetro de escala do efeito aleatório, (e) perturbação na variável resposta e (f) perturbação na variável explicativa. . . . . . . . . . . . . . . . p. 121

B.1 Ponderação de Casos (a) perturbação $y(57)=10$ e (b) perturbação $y(33)=$ $y(81)=19 \ldots \ldots \ldots \ldots \ldots \ldots \ldots \ldots \ldots \ldots$

B.2 Perturbação no parâmetro de escala (a) perturbação $y(57)=10$ e (b) per-

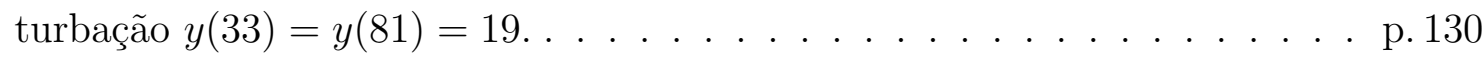

B.3 Perturbação no parâmetro de assimetria (a) perturbação $y(57)=10$ e (b) perturbação $y(33)=y(81)=19 \ldots \ldots \ldots \ldots \ldots$. . . . . . . . . . . . . . . .

B.4 Perturbação na variável resposta (a) perturbação $y(57)=10$ e (b) perturbação $y(33)=y(81)=19 \ldots \ldots \ldots \ldots \ldots$. . . . . . . . . . . . . . . .

B.5 Perturbação na variável explicativa (a) perturbação $y(57)=10$ e (b) perturbação $y(33)=y(81)=19 \ldots \ldots \ldots \ldots \ldots$. . . . . . . . . . . . . . . . .

B.6 Influência global $\left(L D_{i}^{c}\right)$ (a) perturbação $y(57)=10$ e (b) perturbação

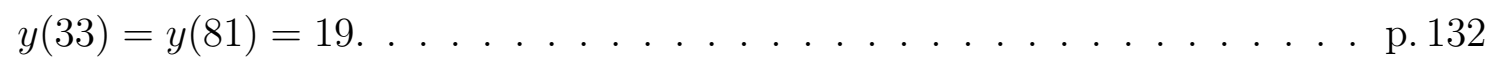

B.7 Influência global $\left(D_{i}^{c}\right)$ (a) perturbação $y(57)=10$ e (b) perturbação $y(33)=$

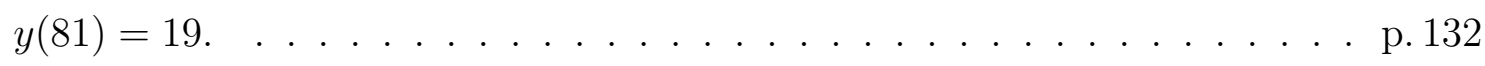

C.1 Dados AIS. Perturbação de ponderação de casos nos modelos assimétricos:

(a) normal (b) t de Student (c) exponencial potência e (d) slash. . . . . . . p. 134 
C.2 Dados AIS. Perturbação no parâmetro de escala nos modelos assimétricos:

(a) normal (b) t de Student (c) exponencial potência e (d) slash. . . . . . . p. 135

C.3 Dados AIS. Perturbação na variável resposta nos modelos assimétricos: (a) normal (b) t de Student (c) exponencial potência e (d) slash. . . . . . . . . p. 135

C.4 Dados AIS. Perturbação em uma variável explicativa nos modelos assimétricos:

(a) normal (b) t de Student (c) exponencial potência e (d) slash. . . . . . . p. 136

C.5 Dados AIS. Perturbação no parâmetro de assimetria nos modelos assimétricos:

(a) normal (b) t de Student (c) exponencial potência e (d) slash. . . . . . . p. 136

C.6 Dados AIS. Alavanca nos modelos assimétricos: (a) normal (b) t de Student

(c) exponencial potência e (d) slash. . . . . . . . . . . . . . . . . . p. 137

C.7 Dados AIS. Influência global ( $L D i)$ nos modelos assimétricos: (a) normal (b) t de Student (c) exponencial potência e (d) slash. . . . . . . . . . . . . p. 137

C.8 Dados AIS. Influência global ( $D i$ ) nos modelos assimétricos: (a) normal (b) t de Student (c) exponencial potência e (d) slash. . . . . . . . . . . . p. 138 


\section{Lista de Tabelas}

2.1 Modelo de regressão NA com dados simulados: Estimativas dos parâmetros do modelo, com seus respectivos erros padrão assintóticos entre parênteses. p. 30

2.2 Conjunto de dados sobre qualidade de vida: Estatísticas descritivas para o índice de massa corpórea (imc) e componente física do índice de qualidade de vida (pcs) dos indivíduos (DP é o desvio-padrão) . . . . . . . . . . . p. 33

2.3 EMVs do modelo normal e modelo normal assimétrico (NA) para o conjunto de dados sobre qualidade de vida (erro padrão estimado assintótico entre parênteses $\ldots \ldots \ldots \ldots \ldots \ldots$. . . . . . . . . . . . . . . . . . . . . . . . . . . . . .

3.1 Modelos de regressão normal simétrico, normal assimétrico (NA) e t-normal assimétrico (TNA) com dados simulados: Estimativas dos parâmetros dos modelos, com seus respectivos erros padrão assintóticos entre parênteses. p.49

3.2 Dados simulados. Mudança nas estimativas dos parâmetros (em \%), excluindo os pontos $(57),(33$ e 81$)$ e $(33,57$ e 81$) \ldots \ldots$. . . . . . 50

3.3 Modelo de regressão TNA simulado: Estimativas do modelo, com e sem perturbações, com seus respectivos erros padrão assintóticos entre parênteses. p. 51

3.4 Pontos influentes sob influência global e local, para o modelo de regressão TNA simulado, com e sem perturbações . . . . . . . . . . . p. 53

3.5 Conjunto de dados AIS: Estatísticas descritivas para as variáveis "soma de manchas na pele"(ssf) e "porcentagem de gordura corporal"(bfat) dos indivíduos (EP é o erro padrão) . . . . . . . . . . . . . . . . . . . p.54

3.6 EMVs do modelo normal simétrico, modelo normal assimétrico (NA) e modelo t-normal assimétrico (TNA) para o conjunto de dados AIS (erro padrão estimado assintótico entre parênteses $) \ldots \ldots \ldots$. . . . . . . . . . 55 
4.1 Modelo normal assimétrico: média das $M$ estimativas $\left(\overline{\widehat{\boldsymbol{\theta}}}=\sum_{k=1}^{M} \widehat{\boldsymbol{\theta}}_{k} / M\right)$, erro padrão assintótico obtido através da MI $\left(E P(\widehat{\boldsymbol{\theta}})=\left[\operatorname{Diag}\left(\mathbf{I}_{-1}(\widehat{\boldsymbol{\theta}})\right)\right]^{1 / 2}\right)$ e erro padrão empírico das $M$ estimativas $\left(E P_{e m p}=\left[\sum_{k=1}^{M}\left(\widehat{\boldsymbol{\theta}}_{k}-\overline{\widehat{\boldsymbol{\theta}}}\right)^{2} / M\right]^{1 / 2}\right)$. p. 79

4.2 Modelo t de Student generalizado normal assimétrico: média das $M$ estimativas $\left(\overline{\boldsymbol{\theta}}=\sum_{k=1}^{M} \widehat{\boldsymbol{\theta}}_{k} / M\right)$, erro padrão assintótico obtido através da MI $\left(E P(\widehat{\boldsymbol{\theta}})=\left[\operatorname{Diag}\left(\mathbf{I}_{-1}(\widehat{\boldsymbol{\theta}})\right)\right]^{1 / 2}\right)$ e erro padrão empírico das $M$ estimativas $\left(E P_{e m p}=\left[\sum_{k=1}^{M}\left(\widehat{\boldsymbol{\theta}}_{k}-\overline{\widehat{\boldsymbol{\theta}}}\right)^{2} / M\right]^{1 / 2}\right) \ldots \ldots \ldots \ldots \ldots \ldots$ p. 80

4.3 Modelo slash assimétrico: média das $M$ estimativas $\left(\overline{\boldsymbol{\theta}}=\sum_{k=1}^{M} \widehat{\boldsymbol{\theta}}_{k} / M\right)$, erro padrão assintótico obtido através da MI $\left(E P(\widehat{\boldsymbol{\theta}})=\left[\operatorname{Diag}\left(\mathbf{I}_{-1}(\widehat{\boldsymbol{\theta}})\right)\right]^{1 / 2}\right) \mathrm{e}$ erro padrão empírico das $M$ estimativas $\left(E P_{e m p}=\left[\sum_{k=1}^{M}\left(\widehat{\boldsymbol{\theta}}_{k}-\overline{\widehat{\boldsymbol{\theta}}}\right)^{2} / M\right]^{1 / 2}\right)$. p. 80

4.4 Modelo normal contaminado assimétrico: média das $M$ estimativas $(\overline{\boldsymbol{\theta}}=$ $\left.\sum_{k=1}^{M} \widehat{\boldsymbol{\theta}}_{k} / M\right)$, erro padrão assintótico obtido através da $\operatorname{MI}\left(\operatorname{EP}(\widehat{\boldsymbol{\theta}})=\left[\operatorname{Diag}\left(\mathbf{I}_{-1}(\widehat{\boldsymbol{\theta}})\right)\right]^{1 / 2}\right)$ e erro padrão empírico das $M$ estimativas $\left(E P_{e m p}=\left[\sum_{k=1}^{M}\left(\widehat{\boldsymbol{\theta}}_{k}-\overline{\widehat{\boldsymbol{\theta}}}\right)^{2} / M\right]^{1 / 2}\right)$. p. 80

4.5 Modelo exponencial potência assimétrico: média das $M$ estimativas $(\overline{\boldsymbol{\theta}}=$ $\left.\sum_{k=1}^{M} \widehat{\boldsymbol{\theta}}_{k} / M\right)$, erro padrão assintótico obtido através da $\operatorname{MI}\left(\operatorname{EP}(\widehat{\boldsymbol{\theta}})=\left[\operatorname{Diag}\left(\mathbf{I}_{-1}(\widehat{\boldsymbol{\theta}})\right)\right]^{1 / 2}\right)$ e erro padrão empírico das $M$ estimativas $\left(E P_{e m p}=\left[\sum_{k=1}^{M}\left(\widehat{\boldsymbol{\theta}}_{k}-\overline{\widehat{\boldsymbol{\theta}}}\right)^{2} / M\right]^{1 / 2}\right)$. p. 81

4.6 Médias de tempo e do número de iterações para cada modelo assimétrico (normal, slash, exponencial potência, t de Student generalizado e normal contaminado) nas M replicações. . . . . . . . . . . . . . . . . . . . p. 81

4.7 EMV, AIC e BIC dos 5 modelos ajustados ao conjunto de dados AIS. Os valores entre parênteses são os erros padrão estimados assintóticos (obtidos através da matriz de informação de Fisher observada) . . . . . . . . . . . . p.90

5.1 Dados de colesterol de Framingham: Estatísticas descritivas para os níveis de colesterol e de idade dos indivíduos. . . . . . . . . . . . . . . . p. 119 
5.2 Estimativas dos parâmetros do modelos de regressão mistos de misturas de escala normal assimétricos para os dados de colesterol. Estimativas dos erros padrão assintóticos, através da aproximação da MI de Louis (1982), entre parênteses. . . . . . . . . . . . . . . . . . . . . . p. 119 


\section{Introducão}

\section{Motivação}

A distribuição normal e os modelos de regressão normais têm sido de grande utilidade em estatística. Entretanto, existem indicações de que a hipótese de normalidade não se aplica em certas situações, por exemplo, quando a distribuição é sensível à presença de observações extremas. Distribuições alternativas têm sido consideradas, como por exemplo, t de Student, logística, exponencial potência e normal contaminada, que são membros particulares da classe de distribuições simétricas. Veja, por exemplo, Fang et al. (1990) e Fang e Zhang (1990). Essa classe de distribuições simétricas contém muitas distribuições com caudas mais pesadas que as da distribuição normal.

Modelos simétricos lineares têm sido investigados por vários autores. Por exemplo, Lange, Little e Taylor (1989) apresentam um modelo de regressão sob erros t de Student. Galea et al. (1997) e Liu (2000) discutem métodos de diagnóstico para modelos simétricos de regressão linear multivariados, enquanto Galea et al.(2003) apresentam alguns estudos de diagnóstico para modelos univariados simétricos.

Embora a classe de distribuições simétricas represente a melhor proposta alternativa em relação à distribuição normal, a mesma não é apropriada em situações onde a distribuição amostral do dados observados é assimétrica. Por exemplo, Hill e Dixon (1982) discutem e apresentam exemplos com estruturas assimétricas.

De um ponto de vista prático, muitos autores têm usado transformações de variáveis para alcançar normalidade, e em muitas situações seus trabalhos são satisfatórios. Entretanto, Azzalini e Capitanio (1999) têm apontado alguns problemas. Por exemplo, as variáveis transformadas são mais difíceis de lidar com sua interpretação, especialmente quando cada variável é transformada usando uma função diferente. 
Em situações práticas, onde a usual suposição de normalidade não é satisfeita devido à falta de simetria dos dados, propõe-se como alternativa a utilização de uma família mais geral de distribuições, de forma que se consiga modelar a assimetria dos dados e além disso, incluir a distribuição normal como um caso particular. Esta família de distribuições é denominada normal assimétrica.

Assim, desenvolver estudos de validação e de diagnóstico em modelos assimétricos é de vital importância para situações onde a suposição usual de normalidade não é satisfeita.

A distribuição normal assimétrica univariada surgiu independentemente em vários artigos estatísticos, entre os principais trabalhos pode-se destacar Roberts (1966), O'Hagan e Leonard (1976) e Aigner et al. (1977). Entretanto, foi Azzalini (1985) que apresentou formalmente esta distribuição, estudou suas propriedades e mostrou que a distribuição tem problemas na estimação do parâmetro que controla a assimetria, pelos métodos de estimação usuais (métodos dos momentos e de máxima verossimilhança). Métodos alternativos têm sido estudados por Sartori (2003) na abordagem clássica, e na abordagem bayesiana, por Liseo e Loperfido (2006). Estes últimos propõem a utilização da distribuição a priori de Jeffreys, entretanto esta distribuição tem uma expressão difícil de trabalhar.

Recentemente, Azzalini (2005) apresentou uma discussão em distribuições normais assimétricas com aplicações em modelos de regressão. Bauwens e Laurent (2004) consideram um estudo em modelos GARCH. Vilca-Labra e Leiva-Sánchez (2005) consideram uma extensão da distribuição de Birnbaum-Saunders sob estruturas assimétricas. No contexto de modelos lineares, Lachos et al. (2007a) consideram uma aplicação de técnicas de diagnóstico em modelos mistos lineares.

Generalizações para o caso multivariado dessas idéias têm sido propostas por vários autores, por exemplo, Azzalini e Dalla-Valle (1996), Azzalini e Capitanio (1999), Genton et al. (2001), Sahu et al. (2003) e Gupta e Gupta (2004). Estes últimos autores obtiveram os momentos de um vetor aleatório com distribuição normal assimétrica multivariada e de suas formas quadráticas.

No contexto teórico, estudos sobre distribuições assimétricas estão sendo desenvolvidos por vários autores. Arnold e Beaver (2000) estudaram a distribuição Cauchy assimétrica, enquanto Jones e Faddy (2003) propõem uma extensão de modelos assimétricos para a distribuição $t$ de Student. Arellano-Valle et al. (2004, 2005c) estudam inferência estatística 
em distribuições assimétricas e propõem uma classe mais geral dessas distribuições. ArellanoValle e Azzalini (2006) unificam as famílias de distribuições normais assimétricas.

Aplicações de modelos específicos têm sido desenvolvidas, por exemplo, em modelos mistos. Arellano-Valle et al. (2005a) estudaram modelos lineares mistos com efeitos aleatórios e modelos com erros seguindo uma distribuição normal assimétrica e Arellano-Valle et al. (2007) desenvolveram inferência bayesiana nos modelos assimétricos normais multivariados, obtendo estimativas robustas em modelos mistos.

No contexto de aplicações dos modelos teóricos desenvolvidos, é importante estudar a robustez dos resultados obtidos no processo de estimação dos parâmetros. Surge então a importância da metodologia de análise de diagnóstico desses modelos ajustados. Assim, ajustado um modelo qualquer, utiliza-se a análise de resíduos para estudar a presença de observações "extremas", verificar a hipótese de homogeneidade de variâncias, entre outros fatores.

Ainda no contexto de análise de diagnóstico, investiga-se em um modelo ajustado, observações cujo resíduo é de grande magnitude comparado com as outras observações extremas, observações fora da massa de dados do conjunto das variáveis preditoras (contexto de regressão) (pontos de alavanca) e também investiga-se a ocorrência de observações que influenciam fortemente nas estimativas dos parâmetros do modelo ajustado. A distância de Cook (Cook, 1977) avalia a mudança na estimativa dos coeficientes do modelo quando a i-ésima observação é retirada do conjunto.

A metodologia de influência local, inicialmente proposta por Cook (1986), tem se tornado uma ferramenta popular de diagnóstico para identificação conjunta de observações influentes em regressão linear e não-linear, com a grande vantagem de poder ser aplicada a qualquer modelo paramétrico. Em particular, para modelos lineares com efeitos mistos, Beckman et al. (1987) aplicam este método para detectar observações influentes em um modelo linear misto com erros normais e ênfase na influência de observações individuais. Por outro lado, Lesaffre e Verbeke (1998) consideram a influência local em modelos lineares mistos normais sob o esquema de ponderação de casos, e mais recentemente, Lee e Xu (2004) estudam a influência local em modelos com efeitos mistos não-lineares sob normalidade. Exemplos da aplicação do método de influência local em modelos lineares mistos não-normais são descritos em Ouwens et al. (2001) e Zhu e Lee (2003), que estudam avaliação de influência em modelos lineares 
generalizados mistos. Recentemente, Lachos et al. (2006) aplicam o método de influência local para modelos de Grubbs. Lachos et al. (2008) apresentam um estudo de inferência e influência local em modelos com erros de medição normais assimétricos.

A metodologia de influência local é baseada na medida do afastamento pela verossimilhança, que avalia a influência local das observações nas estimativas dos parâmetros. As matrizes apropriadas para construir os gráficos de influência são dadas em expressões de forma fechada. Essa metodologia consiste em fazer perturbações no modelo, por exemplo, na estrutura de variância, em determinadas variáveis explicativas, na variável resposta e ainda como ponderação de casos, de forma a avaliar que observações são mais influentes em relação ao tipo de perturbação considerado. Outros métodos de influência local surgiram, como o método de Billor e Loynes (1993), Wu e Luo (1993), Poon e Poon (1999) e Farrel e Cadigan (2000).

Na literatura estatística existem poucos trabalhos aplicados sobre análise de influência local e global em modelos lineares assimétricos, onde se baseia nosso objetivo desse estudo.

\section{Diagnóstico de Influência}

Em estudos de modelagem estatística, uma etapa importante corresponde à validação das pressuposições do modelo mediante estudos de sensibilidade. A análise de diagnósticos tem o intuito de verificar possíveis afastamentos das suposições feitas para o modelo (parte sistemática e aleatória), verificar a existência de observações extremas com interferência desproporcional no ajuste e detectar observações influentes nas estimativas do modelo.

A análise de resíduos busca detectar pontos extremos influentes na estimação dos modelos e também adequação do modelo. Técnicas gráficas (como construção de envelope simulado) auxiliam na busca em detectar pontos extremos à distribuição dos dados. Por último, análise de influência busca localizar observações influentes nas estimativas do modelo, feita através do método de alavanca, influência global e local.

A análise de influência global, via análise de eliminação, exclusão de casos, em que o impacto de deletar uma (ou um grupo) observação na estimativa do parâmetro é diretamente avaliada por métricas como a distância entre as verossimilhanças e a distância de Cook (Cook, 1977). Eliminação de casos é a ferramenta mais popular para avaliar o impacto individual de

Ferreira, C. S. 
casos no processo de estimação, sendo considerada como uma medida global de influência.

A análise de influência local, é baseada em geometria diferencial, comparando estimativas de parâmetros antes e depois de perturbar os dados ou as hipóteses do modelo (Cook, 1986). A metodologia de influência local é útil para verificar as suposições do modelo, assim como a identificação de dados aberrantes e/ou influentes, por meio de estudar o efeito de introduzir pequenas perturbações no modelo (ou dados) usando uma medida de influência apropriada. Esta etapa permite estudar suposições de modelos (como homogeneidade de variâncias).

Recentemente, inspirados pela idéia básica do algoritmo EM, Zhu e Lee (2001) propuseram um método unificado para análise de influência local em modelos estatísticos com dados faltantes, através da função de afastamento da verossimilhança completa.

\section{Influência global}

Para avaliar a influência de observações na estimativa do parâmetro $\boldsymbol{\theta}$ de dimensão $p \times 1$ de modelo, algumas estatísticas são de uso comum. Uma, denominada $L D_{i}$, utiliza o afastamento pela log-verossimilhança, dado por

$$
L D_{i}(\boldsymbol{\theta})=2\left[l(\widehat{\boldsymbol{\theta}})-l\left(\widehat{\boldsymbol{\theta}}_{i}\right)\right]
$$

sendo $l(\boldsymbol{\theta})$ a função log-verossimilhança, $\widehat{\boldsymbol{\theta}}$ o estimador de máxima verossimilhança (EMV) de $\boldsymbol{\theta}$ com todos os dados da amostra e $\widehat{\boldsymbol{\theta}}_{i}$ o EMV de $\boldsymbol{\theta}$ com a eliminação da i-ésima observação. Note que $L D_{i}(\boldsymbol{\theta}) \geq 0$.

A segunda medida de influência global, obtida através da eliminação de observações, é a medida de Cook (Cook e Weisberg, 1982) definida como

$$
D_{I}=\frac{\left(\widehat{\boldsymbol{\theta}}-\widehat{\boldsymbol{\theta}}_{I}\right)^{\top} \mathbf{V}\left(\widehat{\boldsymbol{\theta}}-\widehat{\boldsymbol{\theta}}_{I}\right)}{c},
$$

$\operatorname{com} \widehat{\boldsymbol{\theta}}$ e $\widehat{\boldsymbol{\theta}}_{i}$ representando, respectivamente, o EMV de $\boldsymbol{\theta}$ com todos os dados da amostra e com a eliminação do conjunto de observações $I$. A medida $D_{I}$ mede a influência das observações do conjunto $I$ na estimação de $\boldsymbol{\theta}$, segundo a métrica definida por $\mathbf{V}$ e $c$. Neste trabalho, utilizamos $\mathbf{V}$ como sendo a matriz de informação de Fisher observada e $c$ sendo o número de parâmetros do modelo.

Ferreira, C. S. 


\section{Enfoque de influência local de Cook (1986)}

Dado um conjunto de dados observados, introduzimos perturbações no modelo através de um vetor $\boldsymbol{\omega}$ de dimensão $q \times 1$ em algum subconjunto aberto de $\mathbb{R}^{p}$. Geralmente $\boldsymbol{\omega}$ pode refletir qualquer esquema de perturbação bem definido. Por exemplo, $\boldsymbol{\omega}$ pode ser usado para introduzir uma pequena modificação nas variáveis explicativas ou para perturbar a matriz de covariâncias nos erros no modelo de regressão linear (veja Galea et al., 1997).

Supondo que o esquema de perturbação esteja bem definido, denota-se por $l(\boldsymbol{\theta} \mid \boldsymbol{\omega})$ a função log-verossimilhança correspondente ao modelo perturbado. Assume-se que existe um $\boldsymbol{\omega}_{0}$ tal que $l\left(\boldsymbol{\theta} \mid \boldsymbol{\omega}_{0}\right)=l(\boldsymbol{\theta})$, para todo $\boldsymbol{\theta}$. Para avaliar a influência da perturbação $\boldsymbol{\omega}$ sobre o estimador de máxima verossimilhança $\widehat{\boldsymbol{\theta}}$, Cook (1986) sugere estudar o comportamento do afastamento pela log-verossimilhança, definido por

$$
L D(\boldsymbol{\omega})=2\left[l(\widehat{\boldsymbol{\theta}})-l\left(\widehat{\boldsymbol{\theta}}_{\omega}\right)\right]
$$

em torno de $\boldsymbol{\omega}=\boldsymbol{\omega}_{0}$, onde $\widehat{\boldsymbol{\theta}}_{\omega}$ denota o estimador de máxima verossimilhança sob $l(\boldsymbol{\theta} \mid \boldsymbol{\omega})$. Sob esta perspectiva, o gráfico de $L D(\boldsymbol{\omega})$ contém informação essencial sobre a influência do esquema de perturbação. A idéia consiste em analisar como a superfície $\alpha(\boldsymbol{\omega})=\left(\boldsymbol{\omega}^{\top}, L D(\boldsymbol{\omega})\right)^{\top}$ desvia-se de seu plano tangente em $\boldsymbol{\omega}_{0}$. Essa análise pode ser feita estudando as curvaturas das seções normais à superfície $\alpha(\boldsymbol{\omega})$ em $\boldsymbol{\omega}_{0}$, que são interseções de $\alpha(\boldsymbol{\omega})$ com planos contendo o vetor normal ao seu plano tangente em $\boldsymbol{\omega}_{0}$. Na literatura de geometria diferencial, as curvaturas dessas seções normais são denominadas curvaturas normais.

Cook (1986) mostra que a curvatura normal na direção d (com $\|\mathbf{d}\|=1$ ) é dada por

$$
C_{d}(\boldsymbol{\theta})=2\left|\mathbf{d}^{\top} \boldsymbol{\Delta}^{\top} \ddot{\boldsymbol{L}}^{-1} \boldsymbol{\Delta} \mathbf{d}\right|
$$

onde $-\ddot{\boldsymbol{L}}=\frac{\partial^{2} l(\boldsymbol{\theta})}{\partial \boldsymbol{\theta} \partial \boldsymbol{\theta}^{\top}}$ é a matriz de informação observada sob o modelo postulado e $\boldsymbol{\Delta}$ é a matriz de segundas derivadas parciais com respeito a $\boldsymbol{\theta}$ e $\boldsymbol{\omega}$,

$$
\boldsymbol{\Delta}=\frac{\partial^{2} l(\boldsymbol{\theta} \mid \boldsymbol{\omega})}{\partial \boldsymbol{\theta} \partial \boldsymbol{\omega}^{\top}}
$$

avaliadas em $\boldsymbol{\theta}=\widehat{\boldsymbol{\theta}}$ e $\boldsymbol{\omega}=\boldsymbol{\omega}_{0}$.

O resultado na equação acima pode ser utilizado para avaliar a influência que o esquema de perturbações considerado exerce sobre as estimativas dos parâmetros do modelo. Segundo

Ferreira, C. S. 
Cook (1986), a direção que produz a maior mudança local na estimativa dos parâmetros é dada por $\boldsymbol{d}_{\max }$, que corresponde ao autovetor associado ao maior autovalor (em módulo) de $\boldsymbol{\Delta}^{\top} \ddot{\boldsymbol{L}}^{-1} \boldsymbol{\Delta}$. O vetor $\mathbf{d}_{\max }$ é utilizado para identificar observações que podem estar controlando propriedades importantes na análise dos dados.

A metodologia de influência local de Cook (1986) apresenta alguns problemas, como a falta de invariância sob reparametrizações e a falta de uma medida (ponto de corte) para a tomada de decisão sobre pontos influentes.

\section{Enfoque de influência Local de Zhu e Lee (2001)}

Para introduzir a metodologia e algumas notações, segue uma revisão do algoritmo EM. Seja $\mathbf{Y}_{c}=\left(\mathbf{Y}_{o}, \mathbf{Y}_{n}\right)$ um conjunto de dados completos com função densidade $p\left(\mathbf{Y}_{c} \mid \boldsymbol{\theta}\right)$, parametrizado por um parâmetro $\boldsymbol{\theta} \in \boldsymbol{\Theta} \subseteq \mathbb{R}^{p}$, onde $\mathbf{Y}_{o}$ é o vetor de dados faltantes e $\mathbf{Y}_{n}$ é vetor de dados observados, de um amostra de tamanho $n$. De acordo com Zhu e Lee (2001), na maioria das aplicações estatísticas, a função log-verossimilhança dos dados completos

$$
\ell_{c}\left(\boldsymbol{\theta} \mid \mathbf{Y}_{c}\right)=\log \left\{p\left(\mathbf{Y}_{c} \mid \boldsymbol{\theta}\right)\right\}
$$

geralmente tem forma mais simples que a log-verossimilhança dos dados observados

$$
\ell_{o}\left(\boldsymbol{\theta} \mid \mathbf{Y}_{o}\right)=\log \left\{p\left(\mathbf{Y}_{o} \mid \boldsymbol{\theta}\right)\right\}
$$

O algoritmo EM consiste de dois passos: o passo E (Esperança) e o passo M (Maximização). No passo E é calculada

$$
Q\left(\boldsymbol{\theta} \mid \boldsymbol{\theta}^{(r)}\right)=E\left\{\ell_{c}\left(\boldsymbol{\theta} \mid \mathbf{Y}_{c}\right) \mid \mathbf{Y}_{o}, \boldsymbol{\theta}^{(r)}\right\}
$$

onde a esperança é tomada com respeito à distribuição condicional $p\left(\mathbf{Y}_{n} \mid \mathbf{Y}_{o}, \boldsymbol{\theta}^{(r)}\right)$. No passo M, determina-se o valor $\boldsymbol{\theta}^{(r+1)}$ que maximiza $Q\left(\boldsymbol{\theta} \mid \boldsymbol{\theta}^{(r)}\right)$. Sob determinadas condições, a seqüência $\boldsymbol{\theta}^{(r)}$ obtida das iterações do algoritmo EM converge para o estimador de máxima verossimilhança $\widehat{\boldsymbol{\theta}}$.

Considere um vetor de perturbação $\boldsymbol{\omega}=\left(\omega_{1}, \ldots, \omega_{q}\right)^{\top}$ variando em uma região aberta $\boldsymbol{\omega} \in \mathbb{R}^{p}$. Sejam $l_{o}\left(\boldsymbol{\theta}, \boldsymbol{\omega} \mid \mathbf{Y}_{o}\right)$ e $l_{c}\left(\boldsymbol{\theta}, \boldsymbol{\omega} \mid \mathbf{Y}_{c}\right)$ as funções log-verossimilhanças para os dados observados e para os dados completos, respectivamente, para o modelo perturbado. Assume- 
se que existe um $\boldsymbol{\omega}_{0}$ tal que $l_{o}\left(\boldsymbol{\theta}, \boldsymbol{\omega}_{0} \mid \mathbf{Y}_{o}\right)=l_{o}\left(\boldsymbol{\theta} \mid \mathbf{Y}_{o}\right)$ e $l_{c}\left(\boldsymbol{\theta}, \boldsymbol{\omega}_{0} \mid \mathbf{Y}_{c}\right)=l_{c}\left(\boldsymbol{\theta} \mid \mathbf{Y}_{c}\right)$, para todo $\boldsymbol{\theta}$. Cook (1986) considera a função de afastamento da verossimilhança

$$
L D(\boldsymbol{\omega})=2\left[\ell_{o}\left(\widehat{\boldsymbol{\theta}} \mid \mathbf{Y}_{o}\right)-\ell_{o}\left(\widehat{\boldsymbol{\theta}}_{o}(\boldsymbol{\omega}) \mid \mathbf{Y}_{o}\right)\right]
$$

Em modelos mais complicados, onde a função log-verossimilhança é intratável (por exemplo, na presença de integrais), a metodologia acima se torna inviável. Motivados por essa deficiência, Zhu e Lee (2001) propõem a função Q-afastamento como uma alternativa à função $L D(\boldsymbol{\omega})$ :

$$
f_{Q}(\boldsymbol{\omega})=2[Q(\widehat{\boldsymbol{\theta}} \mid \widehat{\boldsymbol{\theta}})-Q(\widehat{\boldsymbol{\theta}}(\boldsymbol{\omega}) \mid \widehat{\boldsymbol{\theta}})]
$$

onde $\widehat{\boldsymbol{\theta}}(\boldsymbol{\omega})$ maximiza a função $Q(\boldsymbol{\theta}, \boldsymbol{\omega} \mid \widehat{\boldsymbol{\theta}})=E\left[\ell_{c}\left(\boldsymbol{\theta}, \boldsymbol{\omega} \mid \mathbf{Y}_{c}\right) \mid \mathbf{Y}_{o}, \widehat{\boldsymbol{\theta}}\right]$. O gráfico de influência é definido como $\boldsymbol{\alpha}(\boldsymbol{\omega})=\left(\boldsymbol{\omega}^{\top}, f_{Q}(\boldsymbol{\omega})\right)^{\top}$. Sendo $\boldsymbol{\theta}_{0}$ o verdadeiro valor do parâmetro e se o maior autovalor da matriz semidefinida positiva $I_{o b s}^{-1 / 2} I_{f} I_{o b s}^{-1 / 2}$ converge para 0 em probabilidade, Zhu e Lee (2001) demonstram que

$$
2\left[Q(\widehat{\boldsymbol{\theta}} \mid \widehat{\boldsymbol{\theta}})-Q\left(\boldsymbol{\theta}_{0} \mid \widehat{\boldsymbol{\theta}}\right)\right] \stackrel{L}{\rightarrow} \chi_{p}^{2}
$$

onde $\stackrel{L}{\rightarrow}$ denota convergência em distribuição, $I_{o b s}$ é a matriz de informação observada e $I_{f}$ é a matriz de informação dos dados faltantes.

Seguindo a aproximação desenvolvida em Zhu e Lee (2001), a curvatura normal $C_{f_{Q}, \mathbf{d}}$ de $\boldsymbol{\alpha}(\boldsymbol{\omega})$ próximo a $\boldsymbol{\omega}_{0}$ na direção de -um vetor unitário $\mathbf{d}$ pode ser usada para resumir o comportamento local da função Q-afastamento. Pode ser mostrado que (veja Zhu e Lee, 2001)

$$
C_{f_{Q}, \mathbf{d}}=-2 \mathbf{d}^{\top} \ddot{Q} \boldsymbol{\omega}_{o} \mathbf{d}, \quad-\ddot{Q}_{\boldsymbol{\omega}_{0}}=\boldsymbol{\Delta}_{\boldsymbol{\omega}_{o}}^{\top}\left[-\ddot{Q}_{\boldsymbol{\theta}}(\widehat{\boldsymbol{\theta}})\right]^{-1} \boldsymbol{\Delta}_{\boldsymbol{\omega}_{0}}
$$

onde $\ddot{Q}_{\boldsymbol{\theta}}(\widehat{\boldsymbol{\theta}})=\left.\frac{\partial^{2} Q(\boldsymbol{\theta} \mid \widehat{\boldsymbol{\theta}})}{\partial \boldsymbol{\theta} \partial \boldsymbol{\theta}^{\top}}\right|_{\boldsymbol{\theta}=\widehat{\boldsymbol{\theta}}}$ e $\Delta_{\boldsymbol{\omega}}=\left.\frac{\partial^{2} Q(\boldsymbol{\theta}, \boldsymbol{\omega} \mid \widehat{\boldsymbol{\theta}})}{\partial \boldsymbol{\theta} \partial \boldsymbol{\omega}^{\top}}\right|_{\boldsymbol{\theta}=\widehat{\boldsymbol{\theta}}(\boldsymbol{\omega})}$.

A curvatura normal conformal $B_{f_{Q}, \mathbf{d}}$ para $\boldsymbol{\omega}_{0}$ em uma direção unitária $\mathbf{d}$ é definida por

$$
B_{f_{Q}, \mathbf{d}}=\frac{-2 \mathbf{d}^{\top} \ddot{Q} \boldsymbol{\omega}_{0} \mathbf{d}}{\operatorname{tr}\left(-2 \ddot{Q} \boldsymbol{\omega}_{0}\right)}
$$

Sob condições de regularidade, $\ddot{Q}_{\boldsymbol{\omega}_{0}}$ é semidefinida positiva. Ainda, Zhu e Lee (2001) 
provam que $0 \leq B_{f_{Q}, \mathbf{d}} \leq 1$. Como em Cook (1986), a expressão $-\ddot{Q} \boldsymbol{\omega}_{0}$ é a matriz fundamental para detectar observações influentes. Para tanto, considera-se a decomposição espectral de $-\ddot{Q} \omega_{0}$ dada por

$$
-2 \ddot{Q} \boldsymbol{\omega}_{o}=\sum_{k=1}^{q} \lambda_{k} \mathbf{e}_{k} \mathbf{e}_{k}^{\prime},
$$

onde $\left(\lambda_{1}, \mathbf{e}_{1}\right), \ldots,\left(\lambda_{q}, \mathbf{e}_{q}\right)$ são os pares (autovalores-autovetores) da matriz $-2 \ddot{Q} \boldsymbol{\omega}_{o} \operatorname{com} \lambda_{1} \geq$ $\ldots \geq \lambda_{r}, \lambda_{r+1}=\ldots=\lambda_{q}=0 \mathrm{e} \mathbf{e}_{1}, \ldots, \mathbf{e}_{q}$ são os vetores da base ortonormal associada. Lesaffre e Verbeke (1998), Poon e Poon (1999) propuseram inspecionar todas $C_{f_{Q}, \mathbf{u}_{j}}$, onde $\mathbf{u}_{j}$ é um vetor de perturbação básica, com $j$-ésima entrada igual a 1 e restantes iguais a 0 . Seja $\widehat{\lambda}_{k}=\lambda_{k} /\left(\lambda_{1}+\ldots+\lambda_{q}\right)$. Desde que $\operatorname{tr}\left(-2 \ddot{Q}_{\boldsymbol{\omega}_{0}}\right)=\sum_{i=1}^{r} \lambda_{i}$, pode ser visto que

$$
C_{f_{Q}, \mathbf{u}_{j}}=\sum_{i=1}^{r} \lambda_{i} e_{i j}^{2}, \quad B_{f_{Q}, \mathbf{u}_{j}}=\sum_{i=1}^{r} \widetilde{\lambda}_{i} e_{i j}^{2} .
$$

Assim, tem-se que $B_{f_{Q}, \mathbf{e}_{k}}=\widetilde{\lambda}_{k}$. Segundo Zhu e Lee (2001), um autovetor $\mathbf{e}_{i}$ de $-2 \ddot{Q} \boldsymbol{\omega}_{o}$ é chamado $m_{0}$-influente se $B_{f_{Q}, \mathbf{e}_{i}} \geq m_{0} / r$. Considerando $\mathbf{e}_{k}^{2}=\left(e_{k 1}^{2}, \ldots, e_{k q}^{2}\right)$, define-se como vetor de contribuição agregada de todos os autovetores $m_{0}$-influentes a soma ponderada

$$
M\left(m_{0}\right)=\sum_{i: \widetilde{\lambda}_{i} \geq m_{0} / r} \widehat{\lambda}_{i} \mathbf{e}_{i}^{2}
$$

Em particular, quando $m_{0}=0, M(0)=\sum_{i=1}^{r} \widetilde{\lambda}_{i} \mathbf{e}_{i}^{2}$ e $M(0)_{j}=B_{f_{Q}, \mathbf{u}_{j}}$, para todo $j$ e sua média é $\overline{M(0)}=1 / q$. Ver Zhu e Lee (2001) para outras propriedades teóricas de $B_{f_{Q}, \mathbf{u}_{l}}$, tal como a invariância sob reparametrização de $\boldsymbol{\theta}$.

Assim, a avaliação de casos influentes é baseada em $\left\{M(0)_{l}, l=1, \ldots, q\right\}$. Zhu e Lee (2001) propõem como ponto de corte o valor $\overline{M\left(m_{0}\right)}+c^{*} D P\left(M\left(m_{0}\right)\right)$, onde $D P\left(m_{0}\right)$ é o desvio padrão de $\left\{M\left(m_{0}\right)_{l}, l=1, \ldots, q\right\}$ e $c^{*}$ é uma constante arbitrária. Adicionalmente, Lee e $\mathrm{Xu}(2004)$ propõem usar $1 / n+c^{*} D P(0)$ como um ponto de corte para considerar as observações influentes.

Por último, diagnósticos via exclusão de casos são também considerados neste trabalho. Medidas de diagnóstico são desenvolvidas onde o vetor $\left(\mathbf{y}_{i}^{\top}, \mathbf{X}_{i}^{\top}\right)$ é excluído. Na literatura, as medidas clássicas são a distância de Cook e o afastamento pela verossimilhança. Baseado nessas idéias, Lee e Xu (2004) propõem as medidas análogas $D_{i}^{c}$ e $\left(L D_{i}^{c}\right)$ para a função Q. 
Essas medidas são, respectivamente, dadas por

$$
\begin{gathered}
D_{i}^{c}=\left(\widehat{\boldsymbol{\theta}}_{(i)}-\widehat{\boldsymbol{\theta}}\right)^{\top}[-\ddot{Q}(\widehat{\boldsymbol{\theta}} \mid \boldsymbol{\theta})]\left(\widehat{\boldsymbol{\theta}}_{(i)}-\widehat{\boldsymbol{\theta}}\right), \\
L D_{i}^{c}=2\left[Q(\widehat{\boldsymbol{\theta}} \mid \widehat{\boldsymbol{\theta}})-Q\left(\widehat{\boldsymbol{\theta}}_{(i)} \mid \widehat{\boldsymbol{\theta}}\right)\right],
\end{gathered}
$$

onde $\widehat{\boldsymbol{\theta}}_{(i)}$ é o maximizador da função $Q_{(i)}(\boldsymbol{\theta} \mid \widehat{\boldsymbol{\theta}}), i=1, \ldots, n$. Neste trabalho, utiliza-se $M(0)$ como diagnóstico para influência local e $D_{i}^{c}$ e $L D_{i}^{c}$ como medidas de influência global.

\section{Método de Alavanca Generalizado}

Vários autores têm destacado a importância do conceito de ponto de alavanca para diagnóstico em modelos de regressão (Hoaglin e Welsh, 1978; Cook e Weisberg, 1982; Chatterjee e Hadi, 1986 e Andrade, 2004, dentre outros). A idéia principal está na avaliação da influência de $y_{i}$ em seu valor predito (veja, Emerson et al. 1989; Wei et al. 1998). Essa influência pode ser representada pela derivada $\partial \widehat{y}_{i} / \partial y_{i}$. Sob o modelo normal linear, $\partial \widehat{y}_{i} / \partial y_{i}=h_{i i}$, sendo que $h_{i i}$ é o $i$-ésimo elemento da diagonal principal da matriz de projeção $\boldsymbol{H}=\boldsymbol{X}\left(\boldsymbol{X}^{\top} \boldsymbol{X}\right)^{-1} \boldsymbol{X}^{\top}$, onde $\boldsymbol{X}$ é a matriz de planejamento do modelo de regressão.

A idéia de ponto de alavanca foi generalizada para modelos mais complexos. Por exemplo, Ross (1987), St. Laurent e Cook (1992) e Wei et al. (1998) estenderam o método de alavanca para modelos não-lineares; Banerjee e Frees (1997) definem matrizes de alavanca para cada indivíduo no contexto de medidas repetidas; Christensen et al. (1992) sugerem uma medida de alavanca para modelos lineares mistos sob normalidade; Paula (1999) considera o método de alavanca em modelos de regressão linear quando o vetor de parâmetros $\mu$ é restrito por desigualdades lineares e, mais recentemente, Nobre e Singer (2006) propõem incorporar a informação dos efeitos aleatórios ajustados e consideram a matriz de alavancas generalizada em modelos lineares com efeitos mistos.

O objetivo principal do método de alavanca é medir a influência da resposta observada no seu próprio valor ajustado. Tipicamente esta medida tem sido definida como uma taxa de mudança instantânea no valor predito relativo ao valor da variável resposta (veja, por exemplo, St. Laurent e Cook, 1992 e Wei et al., 1998).

Considere $\mathbf{Y}$ o vetor de respostas observadas com densidade $f(\mathbf{Y} ; \boldsymbol{\theta})$, em que $\boldsymbol{\theta}$ representa 
um vetor de parâmetros $p$-dimensional. Considerando $\boldsymbol{\mu}=E[\mathbf{Y}]$ expresso como $\boldsymbol{\mu}=\boldsymbol{\mu}(\boldsymbol{\theta})$ e $\widehat{\boldsymbol{\theta}}=\widehat{\boldsymbol{\theta}}(\mathbf{Y})$ o EMV de $\boldsymbol{\theta}, \widehat{\mathbf{Y}}$ representa o vetor de respostas preditas. A matriz de alavancas generalizadas é definida como $\mathbf{G L}(\widehat{\boldsymbol{\theta}})=\partial \widehat{\mathbf{Y}} / \partial \mathbf{Y}^{\top}$. Segue de Wei et al. (1998) que a matriz de alavancas generalizada assume a forma

$$
\mathbf{G L}(\widehat{\boldsymbol{\theta}})=\left.D_{\boldsymbol{\theta}} \mathbf{I}^{-1}(\boldsymbol{\theta}) \ddot{\mathbf{L}}_{\boldsymbol{\theta} \mathbf{Y}}\right|_{\boldsymbol{\theta}=\widehat{\boldsymbol{\theta}}}
$$

onde $\mathbf{I}(\boldsymbol{\theta})$ representa a matriz de informação observada, $D_{\boldsymbol{\theta}}=\frac{\partial \boldsymbol{\mu}}{\partial \boldsymbol{\theta}^{\top}}$ e $\ddot{\mathbf{L}}_{\boldsymbol{\theta} \mathbf{Y}}=\frac{\partial^{2} l(\boldsymbol{\theta})}{\partial \boldsymbol{\theta} \partial \mathbf{Y}^{\top}}$. Aplicações utilizando a matriz de alavancas ocorrem em vários trabalhos ( Zhu et al., 2001; Zhu e Lee, 2001; Fei e Pan, 2003, Zhu e Lee, 2003; Lee e Xu, 2004).

Baseado na proposta de Wei et al. (1998), assim como nos trabalhos de Zhu e Lee (2001) e Zhu et al. (2001), Salgado (2006) define a matriz de alavancas generalizada para modelos com dados incompletos como

$$
\mathbf{G L}(\widehat{\boldsymbol{\theta}})=D_{\boldsymbol{\theta}}\left[-\ddot{Q}_{\boldsymbol{\theta}}(\widehat{\boldsymbol{\theta}})\right]^{-1} \ddot{Q}_{\boldsymbol{\theta}, \mathbf{y}}(\widehat{\boldsymbol{\theta}})
$$

onde $\ddot{Q}_{\boldsymbol{\theta}, \mathbf{y}}(\widehat{\boldsymbol{\theta}})=\left.\frac{\partial^{2} Q_{\boldsymbol{\theta}}(\boldsymbol{\theta} \mid \widehat{\boldsymbol{\theta}})}{\partial \boldsymbol{\theta} \partial \mathbf{y}^{\top}}\right|_{\boldsymbol{\theta}_{\bar{\approx}} \widehat{\boldsymbol{\theta}}}, \ddot{Q}_{\boldsymbol{\theta}}(\widehat{\boldsymbol{\theta}})=\left.\frac{\partial^{2} Q(\boldsymbol{\theta} \mid \widehat{\boldsymbol{\theta}})}{\partial \boldsymbol{\theta} \partial \boldsymbol{\theta}^{\top}}\right|_{\boldsymbol{\theta}=\widehat{\boldsymbol{\theta}}}$, sendo a função $Q$ proveniente da esperança condicional da funçã̃o log-verossimilhança de dados completos no algoritmo EM. Na ausência de dados faltantes, (1.7) reduz-se a $\mathbf{G L}(\widehat{\boldsymbol{\theta}})$ como em (1.6).

Seja $p_{0}=\sum_{j=1}^{K} \mathbf{G L}_{j j}(\widehat{\boldsymbol{\theta}})=\operatorname{tr}(\mathbf{G L}(\widehat{\boldsymbol{\theta}}))$, K denota a dimensão do vetor de respostas observadas Y. Salgado (2006) propõe a medida $c_{0} p_{0} / K$ como um instrumento para comparar os elementos diagonais de $\mathbf{G L}(\widehat{\boldsymbol{\theta}})$, em que a constante positiva $c_{0}$ pode ser escolhida, por exemplo, como $c_{0}=2$ ou 3 (critérios mais objetivos para julgar observações com alta alavancagem ainda são objeto de investigação). Logo, observações com valores $\mathbf{G L}_{j j}(\widehat{\boldsymbol{\theta}})>c_{0} p_{0} / K$ são consideradas como pontos de alavanca.

\section{Técnicas Gráficas}

A construção de envelope simulado é realizada basicamente através da distribuição de probabilidade de alguma função (resíduo ordinário, resíduo Studentizado, formas quadráticas) conhecida do modelo proposto. Para cada observação do conjunto de dados, reamostra-se uma quantidade K fixa (por exemplo, 200, 300, 500) baseado na distribuição conhecida e toma-se a média e dois percentis (neste trabalho, adota-se sempre o quinto e o nonagésimo

Ferreira, C. S.

IME/USP 
quinto) dessas reamostras, afim de traçar linhas para cada uma das estatísticas mencionadas. Um bom ajuste dos dados à distribuição de referência ocorre quando todos os pontos da amostra estão dentro das duas bandas de confiança (percentis 5 e 95).

\section{Objetivos do trabalho}

O objetivo deste trabalho é apresentar um estudo de inferência e diagnóstico em modelos assimétricos. O estudo será baseado em técnicas gráficas (construção de envelope simulado) e análises de influência global, local e alavancagem, através da metodologia de Zhu e Lee (2001) para dados incompletos. São estudados os modelos de regressão normais, t-normais, de mistura de escalas normais e modelos mistos de misturas de escalas normais assimétricos. São utilizados os softwares R (R Development Core Team, 2005) e Matlab (MATLAB for Windows User's Guide, 1991) para as programações dos processos de estimação e diagnóstico dos modelos estudados. Na parte de estimação, embora possam ser usadas técnicas de estimação direta, a obtenção das estimativas de máxima verossimilhança será via algoritmo EM (Dempster et al., 1977), devido à facilidade de escrever os modelos assimétricos em estruturas condicionais, utilizando variáveis latentes. São então relacionados os seguintes objetivos:

1. Apresentar um estudo de diagnóstico, segundo a metodologia de Zhu e Lee (2001), em modelos normais e t-normais assimétricos, juntamente com uma solução numérica via algoritmo EM dos EMV dos parâmetros dos modelos.

2. Apresentar uma nova distribuição de mistura de escalas normais assimétricas, englobando as distribuições simétricas normal, t de Student, normal-contaminada, slash, exponencial potência, bem como uma solução numérica via algoritmo EM dos EMV dos parâmetros dos modelos e ainda um estudo de diagnóstico, via metodologia de Zhu e Lee (2001) nesses modelos.

3. Apresentar uma nova classe de modelos mistos, através de misturas de escalas normais assimétricas, bem como uma solução numérica através do algoritmo EM dos EMV dos parâmetros dos modelos e análise de diagnóstico via metodologia de Zhu e Lee (2001). 


\section{Organização do trabalho}

O Capítulo 1 descreve a distribuição normal assimétrica introduzida por Azzalini (1985, 1986), nas formas padrão e de locação-escala, destacando suas propriedades, momentos, função geradora de momentos, representação estocástica e distribuições de formas quadráticas. Em seguida, um processo de estimação usando algoritmo EM dos parâmetros do modelo de regressão é abordado, através da função de verossimilhança completa do modelo, alcançando uma solução numérica para os estimadores dos parâmetros. Logo após é feito um estudo de diagnóstico através de análises de influência global e local, usando a metodologia desenvolvida por Zhu e Lee (2001), utilizando a função de verossimilhança completa. A matriz hessiana é calculada e são analisadas as perturbações de ponderação de casos, perturbação no parâmetro de escala $\sigma^{2}$, perturbação no parâmetro de assimetria $\lambda$, perturbação nas variáveis resposta e explicativa, e por último, as matrizes para análise de alavancagem são calculadas. Um estudo de simulação, abordando um modelo de regressão com uma variável regressora é realizado, sendo traçados o histograma da variável resposta e o gráfico de envelope simulado para visualização da qualidade do ajuste. Ainda, os gráficos de diagnóstico das perturbações consideradas são traçados, determinando características de pontos influentes no modelo de regressão normal assimétrico. Por último, uma aplicação do modelo é realizada, considerando um conjunto de dados da área de medicina, sendo estudada a análise de diagnóstico para o modelo. Ainda, uma situação de uma modelo normal assimétrico, porém com pontos extremos na cauda pesada da amostra, é simulado e detectando a necessidade de um modelo mais robusto. No Apêndice A são descritas algumas aproximações de funções necessárias para o cálculo da matriz de informação de Fisher esperada para o modelo normal assimétrico.

O Capítulo 2 descreve o modelo t-normal assimétrico, introduzido por Gómez et al. (2007), seus momentos e distribuições de formas quadráticas. Em seguida, um processo de estimação através do algoritmo EM dos parâmetros do modelo de regressão é abordado, usando a função log-verossimilhança completa do modelo, considerando a distribuição $t$ como um produto de uma normal por uma gama invertida e alcançando uma solução numérica para os estimadores dos parâmetros. Logo após é feito um estudo de análise de diagnóstico via influência global e local, através da metodologia desenvolvida por Zhu e Lee (2001), utilizando a função de verossimilhança completa. A matriz hessiana é calculada, e consideradas as perturbações de ponderação de casos, perturbação no parâmetro de escala $\sigma^{2}$, perturbação

Ferreira, C. S.

IME/USP 
no parâmetro de assimetria $\lambda$, perturbação nas variáveis resposta e explicativa, e por último, as matrizes para análise de alavancagem são calculadas. Um estudo de simulação, abordando um modelo de regressão com uma variável regressora é realizado, fazendo exercícios de exclusão e modificação de alguns pontos. Ainda, os gráficos de diagnóstico das perturbações consideradas são traçados, determinando características de pontos influentes no modelo de regressão t-normal assimétrico. Por último, uma aplicação do modelo é realizada, considerando o conjunto de dados dos atletas australianos, freqüentemente estudado nos modelos assimétricos por vários autores, sendo estudada a análise de diagnóstico para o modelo. No Apêndice B é descrita a matriz de informação de Fisher esperada para o modelo t-normal assimétrico. Ainda, gráficos adicionais de diagnóstico para os modelos simulados na Seção 3.5 são apresentados.

O Capítulo 3 apresenta uma nova família de distribuições assimétricas univariadas gerada pelo núcleo da distribuição normal (como função assimétrica) usando, por outro lado, distribuições simétricas da classe das distribuições com mistura de escalas normais (Andrews e Mallows, 1974; Lange e Sinsheimer, 1993), englobando as distribuições normal, t de Student, slash, normal-contaminada e exponencial-potência assimétricas. Propriedades das distribuições são estudadas, como representação estocástica, função geradora de momentos, média e variância, formas quadráticas e linearidade. Um aspecto interessante e simplificador da família é que a implementação do algoritmo EM é facilitada pelo fato de os passos E serem exatamente como na família de modelos normal/ independente (NI), proposta em Lange e Sinsheimer (1993), obtendo uma solução numérica para o estimador dos parâmetros do modelo de regressão. Distribuições condicionais para o algoritmo EM são desenvolvidas. As matrizes de informação observadas são calculadas para cada distribuição. Um estudo de convergência do algoritmo EM proposto é desenvolvido, determinando a eficácia dos estimadores calculados neste trabalho. Logo após é feito um estudo de diagnóstico via influência global e local, através da metodologia desenvolvida por Zhu e Lee (2001), utilizando a função de verossimilhança completa. A matriz hessiana é calculada e analisadas as perturbações de ponderação de casos, perturbação no parâmetro de escala $\sigma^{2}$, perturbação no parâmetro de assimetria $\lambda$, perturbação nas variáveis resposta e explicativa, e por último, as matrizes para análise de alavancagem são calculadas. Um estudo de simulação é realizado, analisando o comportamento de dois estimadores, a média e a mediana amostrais, sob as cinco distribuições consideradas, através da construção dos gráfico de caixas (boxplots). Por último,

Ferreira, C. S. 
uma aplicação do modelo é realizada, considerando o conjunto de dados dos atletas australianos, verificando a distribuição que melhor se ajusta aos dados, através de inspeção visual dos gráficos de envelopes e da melhor qualidade de ajuste, determinada pela função log-verossimilhança. No Apêndice C é dada uma transformação da fdp de uma distribuição de misturas de escala normal assimétrica. Também são apresentados os gráficos de diagnósticos para as distribuições normal, t-normal, slash e exponencial-potência assimétricas.

O Capítulo 4 apresenta uma nova classe de modelos lineares mistos, através da classe de distribuições de misturas de escalas normais simétricas e assimétricas abordadas no Capítulo 3. A representação estocástica do modelo é apresentada, bem como o valor esperado da variável resposta. O algoritmo EM é apresentado, obtendo uma solução numérica para os estimadores e também as distribuições condicionais que auxiliam na implementação do algoritmo EM. Logo após, é feito um estudo de diagnóstico considerando análises de influência global e local, através da metodologia desenvolvida por Zhu e Lee (2001), utilizando a função de verossimilhança completa. A matriz hessiana é calculada, e consideradas as perturbações de ponderação de casos, perturbação no parâmetro de escalas $\sigma_{e}^{2}$ e $\sigma_{b}^{2}$, perturbação no parâmetro de assimetria $\lambda$, perturbação nas variáveis resposta e explicativa. Um estudo de simulação utilizando a distribuição t-normal assimétrica é conduzido, sob perturbação de alguns pontos e verificadas a capacidade do modelo de detectar pontos extremos. Ainda, uma aplicação utilizando os dados de colesterol de Framingham é desenvolvida, ajustando cada distribuição de mistura e aplicando técnicas de diagnóstico àquela que melhor se ajuste aos dados.

Finalmente, no Capítulo 5 são apresentadas algumas considerações finais e perspectivas de trabalhos futuros.

Ferreira, C. S. 


\section{Modelo Normal Assimétrico}

Em muitas situações práticas, onde a suposição usual de normalidade não é satisfeita devido à falta de simetria dos dados, propõe-se como alternativa a utilização de uma família mais geral de distribuições, de forma que se consiga modelar a assimetria dos dados e além disso, incluir a distribuição normal como um caso particular. Esta família de distribuições é denominada normal assimétrica.

\subsection{Distribuição Normal Assimétrica Padrão}

Esta seção apresenta as propriedades da distribuição normal assimétrica uniparamétrica, a qual será denominada distribuição normal assimétrica padrão. Esta distribuição depende apenas de um parâmetro, o qual caracteriza a assimetria da sua função densidade. Ainda nesta seção expomos uma extensão do modelo padrão, acrescentando parâmetros de posição e de escala nos modelos assimétricos normais.

Definição 2.1. Uma variável aleatória $Z$ tem distribuição normal assimétrica padrão se sua função densidade de probabilidade é dada por

$$
f_{Z}(z)=2 \phi(z) \Phi(\lambda z), \quad z \in \mathbb{R}
$$

onde $\phi(\cdot)$ e $\Phi(\cdot)$ são as funções densidade de probabilidade e distribuição de uma normal padrão, respectivamente.

O parâmetro $\lambda$ caracteriza a forma da distribuição e também é denominado parâmetro de assimetria, pois valores negativos de $\lambda$ indicam assimetria negativa e valores positivos de $\lambda$ indicam assimetria positiva. Se $\lambda=0$, a densidade acima coincide com a densidade da distribuição normal padrão e portanto é simétrica. Será utilizada a seguinte notação: 
$Z \sim N A(\lambda)$

A função distribuição associada à densidade (2.1) é denotada por $F_{Z}(z ; \lambda)$ e dada por

$$
F_{Z}(z ; \lambda)=2 \Phi_{2}(z, 0 \mid \Omega), \operatorname{com} \Omega=\left[\begin{array}{cc}
1 & -\rho \\
-\rho & 1
\end{array}\right], \quad \rho=\frac{\lambda}{\sqrt{1+\lambda^{2}}}, \quad z \in \mathbb{R}
$$

sendo $\Phi_{2}(\cdot \mid \Omega)$ a função distribuição de uma normal bivariada com média zero e matriz de covariâncias $\Omega$ (Ver Bazán, 2005).

Através de (2.1) vemos que a função de distribuição da normal assimétrica pode ser obtida facilmente se tivermos acesso a um programa que calcule a distribuição acumulada de uma normal bivariada.

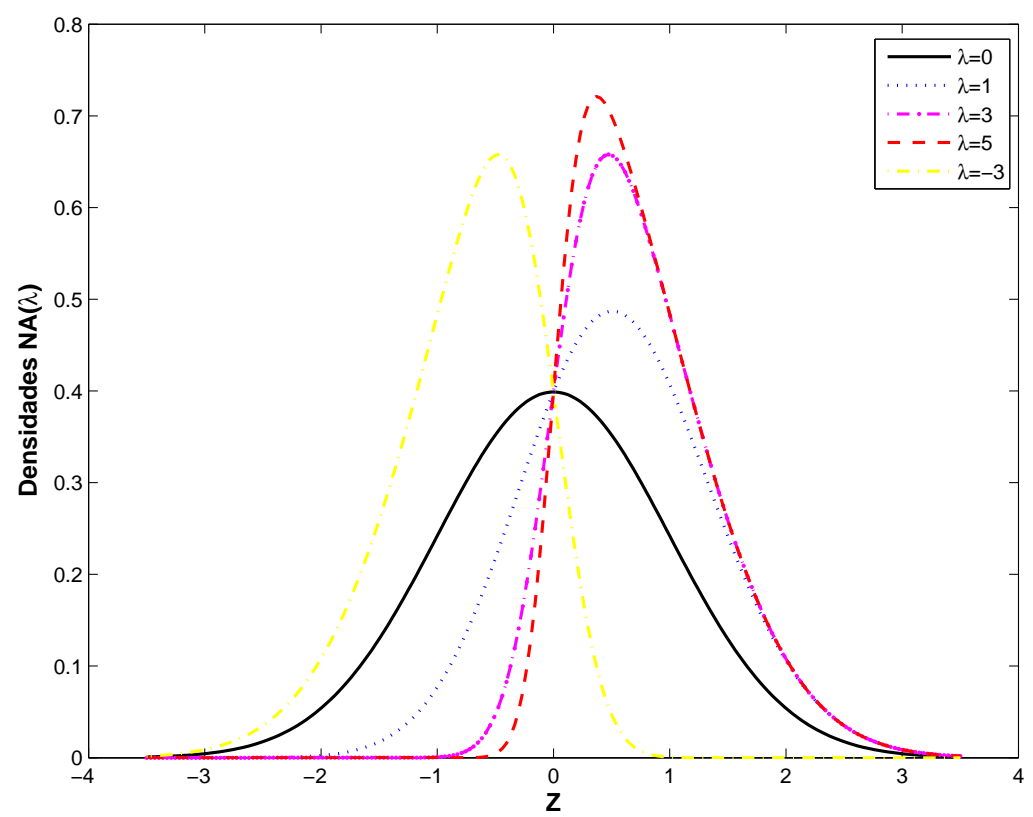

Figura 2.1: Função densidade $N A(\lambda)$, para diferentes valores de $\lambda$.

A densidade em (2.1) possui algumas propriedades interessantes e cujas provas podem ser obtidas em Azzalini (1985) e Azzalini (2004). Entretanto, optamos por destacar algumas propriedades devido a sua importância.

Denotando por $N T\left(\mu, \sigma^{2}\right)$ a distribuição normal truncada à esquerda de zero (Johnson et al., 1994), com parâmetro de locação $\mu$ e de escala $\sigma^{2}$, com densidade dada por $2 \phi(Z) \mathbf{I}_{[0, \infty)}(Z)$, onde $\mathbf{I}_{[A, B]}$ denota função indicadora, tem-se as seguintes propriedades: 


\section{Propriedades}

1. Se $Z \sim N A(\lambda)$, então $|Z| \sim N T(0,1)$;

2. Quando $\lambda \rightarrow \infty$, a densidade (2.1) converge para a densidade de uma $N T(0,1)$;

3. Se $Z \sim N A(\lambda)$, então $-Z \sim N A(-\lambda)$;

4. A densidade (2.1) é log-côncava;

5. $1-F_{Z}(-z ; \lambda)=F_{Z}(z ;-\lambda)$;

6. $F_{Z}(z ; 1)=\{\Phi(z)\}^{2}$;

7. $\sup \left|\Phi(z)-F_{Z}(z)\right|=\pi^{-1} \arctan |\lambda| ;$

8. Se $Z \sim N A(\lambda)$, então $Z^{2} \sim \mathcal{X}_{1}^{2}$;

9. (Representação estocástica de Henze, 1986) Se $U, V \sim N(0,1)$, independentes, então

$$
\frac{\lambda}{\sqrt{1+\lambda^{2}}}|U|+\frac{1}{\sqrt{1+\lambda^{2}}} V \sim N A(\lambda)
$$

Esta última propriedade é útil para gerar amostras da distribuição normal assimétrica a partir da normal padrão.

A seguir obteremos a função geradora de momentos (f.g.m) da distribuição normal assimétrica, da qual derivaremos medidas importantes para a caracterização desta distribuição, tais como, média, variância e coeficiente de assimetria.

A função geradora de momentos da normal assimétrica é dada por

$$
M_{Z}(t)=2 \exp \left(t^{2} / 2\right) \Phi(\rho t)
$$

Os momentos pares de uma distribuição $N A(\lambda)$ são dados por

$$
E\left[Z^{2 k}\right]=2^{-k} \frac{(2 k) !}{k !}
$$

sendo $k$ um inteiro positivo. Os momentos ímpares são dados por

$$
E\left[Z^{2 k+1}\right]=\sqrt{\frac{2}{\pi}} \lambda\left(1+\lambda^{2}\right)^{-\left(k+\frac{1}{2}\right)} 2^{-k}[(2 k+1) !] \sum_{j=0}^{k} \frac{j !(2 \lambda)^{2 j}}{(2 j+1) !(k-j) !} .
$$

Ferreira, C. S. 
Assim, a média e a variância de uma distribuição $N A(\lambda)$ são dadas por

$$
E[Z]=c \rho \quad \text { e } \quad \operatorname{Var}(Z)=1-c^{2} \rho^{2}
$$

com $c=\sqrt{\frac{2}{\pi}}$. O coeficiente de assimetria da distribuição normal assimétrica é dado por

$$
\gamma_{1}=c \rho^{3}\left(1-c^{2} \rho^{2}\right)^{-\frac{3}{2}}\left(\frac{4}{\pi}-1\right)=\frac{\sqrt{2}(4-\pi) \lambda^{3}}{\left[\pi+(\pi-2) \lambda^{2}\right]^{3 / 2}}
$$

O coeficiente $\gamma_{1}$ caracteriza como e quanto a distribuição se afasta da suposição de simetria da normal, sendo uma função crescente em $|\lambda|$. Ainda, temos que $\gamma_{1} \in[-0.99527,0.99527]$, o que limita o estudo de modelagem de dados com um índice de assimetria alto.

O coeficiente de excesso de curtose, expresso por

$$
\gamma_{2}=\frac{8}{\pi^{2}}(\pi-3) \rho^{4}\left(1-c^{2} \rho^{2}\right)^{-2}=\frac{8(\pi-3) \lambda^{4}}{\left[\pi+(\pi-2) \lambda^{2}\right]^{2}}
$$

procura caracterizar o formato da distribuição quanto ao seu achatamento, variando no intervalo [0, 0.86869].

\subsection{Distribuição Normal Assimétrica de Locação-Escala}

O modelo (2.1) é estendido introduzindo parâmetros de locação $\mu \in \mathbb{R}$ e de escala $\sigma>0$. Neste caso, será utilizada a notação $Y \sim N A\left(\mu, \sigma^{2}, \lambda\right)$.

Uma variável aleatória $Y$ tem distribuição normal assimétrica com parâmetros de posição $\mu$ e de escala $\sigma$ se sua função densidade de probabilidade é da forma

$$
f_{Y}(y)=\frac{2}{\sigma} \phi\left(\frac{y-\mu}{\sigma}\right) \Phi\left(\lambda \frac{y-\mu}{\sigma}\right), \quad y \in \mathbb{R} .
$$

É fácil verificar que se $Z \sim N A(\lambda)$ e $Y=\mu+\sigma Z$, então $Y \sim N A\left(\mu, \sigma^{2}, \lambda\right)$.

A função de distribuição correspondente a (2.9), denotada por $F_{Y}(y ; \mu, \sigma, \lambda)$, é dada por

$$
F_{Y}(y ; \mu, \sigma, \lambda)=2 \Phi_{2}\left(\frac{y-\mu}{\sigma}, 0 \mid \Sigma\right), \operatorname{com} \Sigma=\left[\begin{array}{cc}
1 & -\rho \\
-\rho & 1
\end{array}\right], \quad y \in \mathbb{R} .
$$

Seja $Y \sim N A\left(\mu, \sigma^{2}, \lambda\right)$. A função geradora de momentos da normal assimétrica é dada 
por

$$
M_{Y}(t)=2 \exp \left(t \mu+\frac{t^{2} \sigma^{2}}{2}\right) \Phi(\rho \sigma t)
$$

e sua forma quadrática

$$
D=\frac{(Y-\mu)^{2}}{\sigma^{2}} \sim \chi_{1}^{2}
$$

Logo, $E\left[D^{m}\right]=\frac{2^{m} \Gamma(m+1 / 2)}{\sqrt{\pi}}$.

O seguinte lema (dado em Lin et al., 2007) fornece uma forma simples de obter momentos superiores da normal assimétrica sem usar sua função geradora de momentos.

Lema 2.1. Se $Y \sim N A\left(\mu, \sigma^{2}, \lambda\right)$ e $X \sim N\left(\mu, \frac{\sigma^{2}}{1+\lambda^{2}}\right)$ então

(i) $E\left[X^{n+1}\right]=\mu E\left(X^{n}\right)+\frac{\sigma^{2}}{1+\lambda^{2}} \frac{d}{d \mu} E\left(X^{n}\right)$.

(ii) $E\left[Y^{n+1}\right]=\mu E\left[Y^{n}\right]+\sigma^{2} \frac{d}{d \mu} E\left[Y^{n}\right]+c \rho \sigma E\left[X^{n}\right]$.

$$
\text { (iii) } \begin{aligned}
E[Y-E(Y)]^{n+1}= & \sigma^{2} \frac{d}{d \mu} E[Y-E(Y)]^{n}+n \sigma^{2} E[Y-E(Y)]^{n-1} \\
& -[E[Y]-\mu] E[Y-E[Y]]^{n}+c \rho \sigma E[X-E[Y]]^{n}
\end{aligned}
$$

A média e a variância de uma variável aleatória $Z \sim N A\left(\mu, \sigma^{2}, \lambda\right)$ são dadas por

$$
E[Y]=\mu+c \sigma \rho \quad \text { e } \operatorname{Var}[Y]=\sigma^{2}\left(1-c^{2} \rho^{2}\right) .
$$

Proposição 2.1. Sejam $X$ e $Y$ duas variáveis aleatórias tais que $X \sim N A\left(\mu, \sigma^{2}, \lambda\right) e$ $Y=a+b X, a$ e $b \in \mathbb{R}$. Então

$$
Y \sim N A\left(a+b \mu, b^{2} \sigma^{2}, \operatorname{sinal}(b) \lambda\right)
$$

onde $\operatorname{sinal}(x)=1$ se $x \geq 0$ e $\operatorname{sinal}(x)=-1$ se $x<0$.

A prova da Proposição 2.1 pode ser encontrada em Rodríguez (2005). Mais informações sobre propriedades e inferência estatística em modelos normais assimétricos podem ser encontradas em Rodríguez (2005), Gómez (2005) e Lin et al. (2007). 


\subsection{Estimação de Máxima Verossimilhança via Algo- ritmo EM}

Suponha que se tenha um conjunto de $n$ observações independentes, denotadas por $Y_{1}, \ldots, Y_{n}$, onde $Y_{i} \sim N A\left(\mu_{i}, \sigma^{2}, \lambda\right), i=1, \ldots, n$. Associado com a observação $i$, considere um vetor $p \times 1$ de covariáveis $\mathbf{x}_{i}$, através do qual especifica-se o preditor linear $\mu_{i}=\mathbf{x}_{i}^{\top} \boldsymbol{\beta}$, onde $\boldsymbol{\beta}$ é um vetor $p$-dimensional de coeficientes de regressão desconhecidos. Assim, relacionando os dois conjuntos de variáveis, tem-se o modelo

$$
\begin{aligned}
y_{i} & =\beta_{0}+\sum_{k=1}^{p} x_{i k} \beta_{k}+\varepsilon_{i}, \quad i=1, \ldots, n, \\
& =\mathbf{x}_{i}^{\top} \boldsymbol{\beta}+\varepsilon_{i}, \quad \varepsilon_{i} \sim N A\left(0, \sigma^{2}, \lambda\right) .
\end{aligned}
$$

Note que $E\left[\varepsilon_{i}\right]=\sqrt{\frac{2}{\pi}} \frac{\sigma \lambda}{1+\lambda^{2}} \neq 0$, para $\lambda \neq 0$. Em termos de previsão para $Y$, geralmente considera-se $\widehat{Y}_{i}=\widehat{\beta}_{0}+\sum_{k=1}^{p} x_{i k} \widehat{\beta}_{k}$ como preditor de $Y_{i} \mid \mathbf{x}_{i}$. Uma forma de corrigir esta distorção é considerar $\widehat{Y}_{i}=\widehat{\beta}_{0}+\sum_{k=1}^{p} x_{i k} \widehat{\beta}_{k}+\sqrt{\frac{2}{\pi}} \frac{\widehat{\sigma} \widehat{\lambda}}{1+\widehat{\lambda}^{2}}$ como preditor de $Y_{i} \mid \mathbf{x}_{i}$. Outra possibilidade é considerar o modelo centrado (ver Freitas, 2005).

Pode-se verificar que a função log-verossimilhança para $\boldsymbol{\theta}=\left(\boldsymbol{\beta}^{\top}, \sigma^{2}, \lambda\right)^{\top}$ para uma amostra de $n$ observações $\left(y_{1}, \ldots, y_{n}\right)$ é dada por

$$
\begin{aligned}
\ell(\boldsymbol{\theta}) & =\sum_{i=1}^{m} \log \left[2 \phi\left(y_{i} \mid \mathbf{x}_{i}^{\top} \boldsymbol{\beta}, \sigma^{2}\right) \Phi\left(\frac{\lambda\left(y_{i}-\mathbf{x}_{i}^{\top} \boldsymbol{\beta}\right)}{\sigma^{2}}\right)\right] \\
& =\sum_{i=1}^{m} \log \left[2 \int_{0}^{+\infty} \phi\left(y_{i} \mid \mathbf{x}_{i}^{\top} \boldsymbol{\beta}, \sigma^{2}\right) \phi\left(t_{i} \mid \lambda\left(y_{i}-\mathbf{x}_{i}^{\top} \boldsymbol{\beta}\right), \sigma^{2}\right) d t_{i}\right] .
\end{aligned}
$$

Maximizar diretamente a função acima para encontrar as estimativas de máxima verossimilhança dos parâmetros pode ser complicado, devido à presença de integrais na expressão 2.14. Uma saída para este problema é utilizar um procedimento de estimação usando algoritmo EM, uma ferramenta popular para estimação de máxima verossimilhança para modelos com dados incompletos. Mais especificamente, seja y o conjunto de dados observados e $\mathbf{s}$ denotando o conjunto de dados faltantes. O dado completo $\mathbf{y}_{c}=(\mathbf{y}, \mathbf{s})$ é $\mathbf{y}$ aumentado com s. Denota-se por $\ell_{c}\left(\boldsymbol{\theta} \mid \mathbf{y}_{c}\right), \boldsymbol{\theta} \in \boldsymbol{\Theta}$, a função log-verossimilhança dos dados completos e por $Q(\boldsymbol{\theta} \mid \widehat{\boldsymbol{\theta}})=E\left[\ell_{c}\left(\boldsymbol{\theta} \mid \mathbf{y}_{c}\right) \mid \mathbf{y}, \widehat{\boldsymbol{\theta}}\right]$, o valor esperado desta função. Cada iteração do algoritmo EM envolve dois passos, um passo $\mathrm{E}$ e um passo $\mathrm{M}$, definidos como:

Ferreira, C. S. 
- Passo E: Calcule $Q\left(\boldsymbol{\theta} \mid \boldsymbol{\theta}^{(k)}\right)$ como uma função de $\boldsymbol{\theta}$;

- Passo M: Encontre $\boldsymbol{\theta}^{(k+1)}$ tal que $Q\left(\boldsymbol{\theta}^{(k+1)} \mid \boldsymbol{\theta}^{(r)}\right)=\max _{\boldsymbol{\theta} \in \boldsymbol{\Theta}} Q\left(\boldsymbol{\theta} \mid \boldsymbol{\theta}^{(k)}\right)$.

Utilizando a representação estocástica de Henze (1986) (Propriedade 9) e a Proposição 2.1, o modelo de regressão (2.13) acima pode ser escrito como

$$
\begin{aligned}
Y_{i} \mid T_{=} t_{i} & \stackrel{\text { ind }}{\sim} N\left(\mathbf{x}_{i}^{\top} \boldsymbol{\beta}+\frac{\sigma \lambda}{\sqrt{1+\lambda^{2}}} t_{i}, \frac{\sigma^{2}}{1+\lambda^{2}}\right), \\
T_{i} & \stackrel{\text { iid }}{\sim} N T(0,1), \quad i=1, \ldots, n .
\end{aligned}
$$

De $\ell(\boldsymbol{\theta})$ em (2.14), a distribuição conjunta de $y_{i}$ e $t_{i}$ é dada por

$$
f\left(y_{i}, t_{i}\right)=\phi\left(y_{i} \mid \mathbf{x}_{i}^{\top} \boldsymbol{\beta}, \sigma^{2}\right) \phi\left(t_{i} \mid \lambda\left(y_{i}-\mathbf{x}_{i}^{\top} \boldsymbol{\beta}\right), \sigma^{2}\right) \mathbf{I}\left(t_{i}>0\right) .
$$

Seja $\mathbf{y}=\left(y_{1}, \ldots, y_{n}\right)^{\top}$ e $\mathbf{t}=\left(t_{1}, \ldots, t_{n}\right)^{\top}$ e tratando $\mathbf{t}$ como dado faltante, segue que a função log-verossimilhança completa associada com $\mathbf{y}_{c}=\left(\mathbf{y}^{\top}, \mathbf{t}^{\top}\right)^{\top}$ é dada por

$$
\begin{aligned}
\ell_{c}\left(\boldsymbol{\theta} \mid \mathbf{y}_{c}\right) & =\sum_{i=1}^{n} \log f\left(y_{i}, t_{i}\right) \\
& \propto-n \log \sigma^{2}-\frac{1}{2 \sigma^{2}} \sum_{i=1}^{n}\left(y_{i}-\mathbf{x}_{i}^{\top} \boldsymbol{\beta}\right)^{2}-\frac{1}{2 \sigma^{2}} \sum_{i=1}^{n}\left[t_{i}-\lambda\left(y_{i}-\mathbf{x}_{i}^{\top} \boldsymbol{\beta}\right)\right]^{2} \\
& =-n \log \sigma^{2}-\frac{1}{2 \sigma^{2}} \mathbf{t}^{2 \top} \mathbf{1}_{n}+\frac{\lambda}{\sigma^{2}} \mathbf{t}^{\top}(\mathbf{y}-\mathbf{X} \boldsymbol{\beta})-\frac{1+\lambda^{2}}{2 \sigma^{2}}(\mathbf{y}-\mathbf{X} \boldsymbol{\beta})^{\top}(\mathbf{y}-\mathbf{X} \boldsymbol{\beta}),
\end{aligned}
$$

onde $\mathbf{t}^{2}=\left(t_{1}^{2}, \ldots, t_{n}^{2}\right)^{\top}, \mathbf{1}_{n}$ é um vetor de 1's de tamanho $n$ e $\mathbf{X}=\left(\mathbf{x}_{1} \ldots, \mathbf{x}_{n}\right)^{\top}$ é a matriz de planejamento, de dimensão $n \times p$.

De (2.14), tem-se que $T_{i} \mid y_{i} \sim N T\left(\lambda\left(y_{i}-\mathbf{x}_{i}^{\top} \boldsymbol{\beta}\right), \sigma^{2}\right)$. Seja $\widehat{t}_{i}=\mathrm{E}\left[T_{i} \mid \boldsymbol{\theta}=\widehat{\boldsymbol{\theta}}, y_{i}\right]$ e $\widehat{t}^{2}{ }_{i}=$ $\mathrm{E}\left[T_{i}^{2} \mid \boldsymbol{\theta}=\widehat{\boldsymbol{\theta}}, y_{i}\right]$. Então, usando os momentos da distribuição normal truncada (Lachos, 2004), tem-se que

$$
\widehat{t_{i}}=\widehat{\lambda} \widehat{\eta}_{i}+\widehat{\sigma} W_{\Phi_{1}}\left(\frac{\widehat{\lambda} \widehat{\eta}_{i}}{\widehat{\sigma}}\right) \text { e } \widehat{t}^{2}{ }_{i}=\widehat{\lambda}^{2} \widehat{\eta}_{i}^{2}+\widehat{\sigma}^{2}+\widehat{\lambda} \widehat{\sigma} \widehat{\eta}_{i} W_{\Phi_{1}}\left(\frac{\widehat{\lambda} \widehat{\eta}_{i}}{\widehat{\sigma}}\right)
$$

onde $W_{\Phi_{1}}(u)=\phi_{1}(u) / \Phi_{1}(u)$ e $\widehat{\eta}_{i}=\left(y_{i}-\mathbf{x}_{i}^{\top} \widehat{\boldsymbol{\beta}}\right), i=1, \ldots, n$.

Denote por $\boldsymbol{\theta}^{(k)}=\left(\boldsymbol{\beta}^{(k)^{\top}}, \sigma^{2(k)}, \lambda^{(k)}\right)^{\top}$ a estimativa de $\boldsymbol{\theta}$ para a $k$-ésima iteração. Segue que a esperança com respeito a t, condicionada em y, da função log-verossimilhança completa 
(Passo E), tem a forma

$$
\begin{aligned}
Q\left(\boldsymbol{\theta} \mid \widehat{\boldsymbol{\theta}}^{(k)}\right)= & \mathrm{E}\left[\ell_{c}\left(\boldsymbol{\theta} \mid \mathbf{y}_{c}\right) \mid \mathbf{y}, \widehat{\boldsymbol{\theta}}^{(k)}\right] \\
= & -n \log {\sigma^{2}}^{(k)}-\frac{1}{2{\sigma^{2}}^{(k)}} \sum_{i=1}^{n}{\widehat{t^{2}}}_{i}^{(k)}+\frac{\lambda^{(k)}}{{\sigma^{2}}^{(k)}} \sum_{i=1}^{n} \widehat{t}_{i}^{(k)}\left(y_{i}-\mathbf{x}_{i}^{\top} \boldsymbol{\beta}^{(k)}\right) \\
& -\frac{1+\lambda^{(k)^{2}}}{2 \sigma^{2(k)}} \sum_{i=1}^{n}\left(y_{i}-\mathbf{x}_{i}^{\top} \boldsymbol{\beta}^{(k)}\right)^{2} .
\end{aligned}
$$

Portanto, tem-se o seguinte algoritmo EM:

Passo E: Dado $\boldsymbol{\theta}=\widehat{\boldsymbol{\theta}}^{(k)}$, calcule $\widehat{t}_{i}^{(k)}$ e ${\widehat{t^{2}}}^{(k)}{ }^{(k)}$, para $i=1, \ldots, n$, usando (2.16).

Passo M: Atualize $\widehat{\boldsymbol{\theta}}^{(k+1)}$ maximizando $Q\left(\boldsymbol{\theta} \mid \widehat{\boldsymbol{\theta}}^{(k)}\right)$ em $\boldsymbol{\theta}$, que leva às seguintes soluções analíticas:

$$
\begin{aligned}
\widehat{\boldsymbol{\beta}}^{(k+1)} & =\left[\mathbf{X}^{\top} \mathbf{X}\right]^{-1} \mathbf{X}^{\top} \mathbf{y}-\frac{\lambda^{(k)}}{1+\lambda^{(k)^{2}}}\left[\mathbf{X}^{\top} \mathbf{X}\right]^{-1} \mathbf{X}^{\top} \widehat{\mathbf{t}}^{(k)}, \\
{\widehat{\sigma^{2}}}^{(k+1)} & =\frac{1}{2 n}\left[\widehat{\mathbf{t}}^{(k)^{\top}} \mathbf{1}_{n}-2 \lambda^{(k)} \widehat{\mathbf{t}}^{(k)^{\top}}\left(\mathbf{y}-\mathbf{X} \boldsymbol{\beta}^{(k)}\right)+\left(1+\lambda^{(k)^{2}}\right) Q\left(\boldsymbol{\beta}^{(k)}\right)\right], \\
\widehat{\lambda}^{(k+1)} & =\frac{\widehat{\mathbf{t}}^{(k)^{\top}}\left(\mathbf{y}-\mathbf{X} \boldsymbol{\beta}^{(k)}\right)}{Q\left(\boldsymbol{\beta}^{(k)}\right)},
\end{aligned}
$$

onde $Q\left(\boldsymbol{\beta}^{(k)}\right)=\left(\mathbf{y}-\mathbf{X} \boldsymbol{\beta}^{(k)}\right)^{\top}\left(\mathbf{y}-\mathbf{X} \boldsymbol{\beta}^{(k)}\right)$.

Claramente, se $\lambda=0, \widehat{\boldsymbol{\beta}}=\left[\mathbf{X}^{\top} \mathbf{X}\right]^{-1} \mathbf{X}^{\top} \mathbf{y}$ é o EMV de $\boldsymbol{\beta}$ do modelo normal simétrico. Por outro lado, $\lambda=0$ implica $\widehat{\mathbf{t}}^{2}{ }_{i}=\widehat{\sigma^{2}}$, resultando na equação $\widehat{\sigma^{2}}=\frac{1}{2 n}\left[n \widehat{\sigma^{2}}+Q(\widehat{\boldsymbol{\beta}})\right]$, implicando $\widehat{\sigma^{2}}=\frac{Q(\widehat{\boldsymbol{\beta}})}{n}$, coincidindo com o EMV de $\sigma^{2}$ do modelo normal simétrico.

São usados como valores iniciais para $\boldsymbol{\theta}$ no algoritmo os estimadores de momentos (Rodríguez, 2005). O EMV de $\boldsymbol{\theta}$ através do algoritmo EM é uma idéia inovadora desta tese.

\subsection{Matriz de Informação de Fisher Observada}

Considere $Y_{1}, \ldots, Y_{n}$, onde $Y_{i} \sim N A\left(\mu_{i}, \sigma^{2}, \lambda\right), \mu_{i}=\mathbf{x}_{i}^{\top} \boldsymbol{\beta}, i=1, \ldots, n$, onde $\boldsymbol{\beta}$ é um vetor $p$-dimensional de coeficientes de regressão desconhecidos. Então a função logverossimilhança $\ell(\boldsymbol{\theta})=\sum_{i=1}^{n} \ell_{i}(\boldsymbol{\theta})$ é da forma

$$
\ell_{i}(\boldsymbol{\theta})=\log 2+\ell_{1_{i}}(\boldsymbol{\theta})+\log \left[\Phi\left(\ell_{2_{i}}(\boldsymbol{\theta})\right)\right], \quad \boldsymbol{\theta}=\left(\boldsymbol{\beta}^{\top}, \sigma^{2}, \lambda\right)^{\top}
$$

Ferreira, C. S. 
onde $\ell_{1}(\boldsymbol{\theta})=\phi\left(y_{i} \mid \mathbf{x}_{i}^{\top} \boldsymbol{\beta}, \sigma^{2}\right)$ e $\ell_{2_{i}}(\boldsymbol{\theta})=\lambda \frac{y_{i}-\mathbf{x}_{i}^{\top} \boldsymbol{\beta}}{\sigma}$. Então, a primeira derivada de $\ell_{i}(\boldsymbol{\theta})$ é dada por

$$
\frac{\partial \ell_{i}(\boldsymbol{\theta})}{\partial \boldsymbol{\psi}}=\frac{\partial \ell_{1_{i}}(\boldsymbol{\theta})}{\partial \boldsymbol{\psi}}+W_{\Phi}\left(l_{2_{i}}(\boldsymbol{\theta})\right) \frac{\partial \ell_{2_{i}}(\boldsymbol{\theta})}{\partial \boldsymbol{\psi}}, \quad \boldsymbol{\psi}=\boldsymbol{\beta}, \sigma^{2}, \lambda
$$

A segunda derivada é dada por

$$
\frac{\partial^{2} \ell_{i}(\boldsymbol{\theta})}{\partial \boldsymbol{\gamma} \partial \boldsymbol{\psi}^{\top}}=\frac{\partial^{2} \ell_{1_{i}}(\boldsymbol{\theta})}{\partial \boldsymbol{\gamma} \partial \boldsymbol{\psi}^{\top}}+W_{\Phi}\left(\ell_{2_{i}}(\boldsymbol{\theta})\right) \frac{\partial^{2} \ell_{2_{i}}(\boldsymbol{\theta})}{\partial \boldsymbol{\gamma} \partial \boldsymbol{\psi}^{\top}}+W_{\Phi}^{(1)}\left(\ell_{2_{i}}(\boldsymbol{\theta})\right) \frac{\partial \ell_{2_{i}}(\boldsymbol{\theta})}{\partial \boldsymbol{\gamma}} \frac{\partial \ell_{2_{i}}(\boldsymbol{\theta})}{\partial \boldsymbol{\psi}^{\top}}
$$

onde $W_{\Phi}^{(1)}(x)=-W_{\Phi}(x)\left(x+W_{\Phi}(x)\right)$ é a derivada de $W_{\Phi}(x)$.

Portanto, a matriz de informação observada para $\boldsymbol{\theta}$ pode ser escrita como

$$
\begin{gathered}
\mathbf{I}(\boldsymbol{\theta})=I_{1}(\boldsymbol{\theta})+I_{2}(\boldsymbol{\theta}), \\
I_{k}(\boldsymbol{\theta})=\left(\begin{array}{ccc}
I_{\boldsymbol{\beta} \boldsymbol{\beta}}^{k} & I_{\sigma^{2} \boldsymbol{\beta}}^{k} & I_{\lambda \boldsymbol{\beta}}^{k} \\
& I_{\sigma^{2} \sigma^{2}}^{k} & I_{\lambda \sigma^{2}}^{k} \\
& & I_{\lambda \lambda}^{k}
\end{array}\right),
\end{gathered}
$$

para $k=1,2, \boldsymbol{\gamma}, \boldsymbol{\psi}=\boldsymbol{\beta}, \sigma^{2}, \lambda$, onde

$$
\begin{aligned}
I_{\boldsymbol{\beta} \boldsymbol{\beta}}^{1} & =\frac{1}{\sigma^{2}} \mathbf{X}^{\top} \mathbf{X} \\
I_{\sigma^{2} \boldsymbol{\beta}}^{1} & =\frac{1}{\sigma^{4}} \mathbf{X}^{\top}(\mathbf{y}-\mathbf{X} \boldsymbol{\beta}), \\
I_{\sigma^{2} \sigma^{2}}^{1} & =-\frac{n}{2 \sigma^{4}}+\frac{1}{\sigma^{6}}(\mathbf{y}-\mathbf{X} \boldsymbol{\beta})^{\top}(\mathbf{y}-\mathbf{X} \boldsymbol{\beta}), \\
I_{\lambda \boldsymbol{\tau}}^{1} & =\mathbf{0}, \boldsymbol{\tau}=\boldsymbol{\beta}, \sigma^{2}, \lambda,
\end{aligned}
$$

e 


$$
\begin{aligned}
I_{\boldsymbol{\beta} \boldsymbol{\beta}}^{2} & =-\frac{\lambda^{2}}{\sigma^{2}} \mathbf{X}^{\top} \mathbf{D}\left(W_{\Phi}^{(1)}\left[\lambda \frac{(\mathbf{y}-\mathbf{X} \boldsymbol{\beta})}{\sigma}\right]\right) \mathbf{X} \\
I_{\sigma^{2} \boldsymbol{\beta}}^{2} & =-\frac{\lambda}{2 \sigma^{3}} \mathbf{X}^{\top} W_{\Phi}\left[\lambda \frac{(\mathbf{y}-\mathbf{X} \boldsymbol{\beta})}{\sigma}\right]-\frac{\lambda^{2}}{2 \sigma^{4}} \mathbf{X}^{\top} \mathbf{D}\left(W_{\Phi}^{(1)}\left[\lambda \frac{(\mathbf{y}-\mathbf{X} \boldsymbol{\beta})}{\sigma}\right]\right)(\mathbf{y}-\mathbf{X} \boldsymbol{\beta}) \\
I_{\lambda \boldsymbol{\beta}}^{2} & =\frac{1}{\sigma} \mathbf{X}^{\top} W_{\Phi}\left[\lambda \frac{(\mathbf{y}-\mathbf{X} \boldsymbol{\beta})}{\sigma}\right]+\frac{\lambda}{\sigma^{2}} \mathbf{X}^{\top} \mathbf{D}\left(W_{\Phi}^{(1)}\left[\lambda \frac{(\mathbf{y}-\mathbf{X} \boldsymbol{\beta})}{\sigma}\right]\right)(\mathbf{y}-\mathbf{X} \boldsymbol{\beta}) \\
I_{\sigma^{2} \sigma^{2}}^{2} & =-\frac{3 \lambda}{4 \sigma^{5}}(\mathbf{y}-\mathbf{X} \boldsymbol{\beta})^{\top} W_{\Phi}\left[\lambda \frac{(\mathbf{y}-\mathbf{X} \boldsymbol{\beta})}{\sigma}\right]-\frac{\lambda^{2}}{4 \sigma^{6}} Q_{w}(\boldsymbol{\beta}) \\
I_{\lambda \sigma^{2}}^{2} & =\frac{1}{2 \sigma^{3}}(\mathbf{y}-\mathbf{X} \boldsymbol{\beta})^{\top} W_{\Phi}\left[\lambda \frac{(\mathbf{y}-\mathbf{X} \boldsymbol{\beta})}{\sigma}\right]+\frac{\lambda}{2 \sigma^{4}} Q_{w}(\boldsymbol{\beta}) \\
I_{\lambda \lambda}^{2} & =-\frac{1}{\sigma^{2}} Q_{w}(\boldsymbol{\beta})
\end{aligned}
$$

onde $W_{\Phi}^{(1)}(x)=-W_{\Phi}(x)\left(x+W_{\Phi}(x)\right)$ é a derivada de $W_{\Phi}(x), \mathbf{D}(\mathbf{a})$ é a matriz diagonal do vetor a e $Q_{w}(\boldsymbol{\beta})=(\mathbf{y}-\mathbf{X} \boldsymbol{\beta})^{\top} \mathbf{D}\left(W_{\Phi}^{(1)}\left[\lambda \frac{(\mathbf{y}-\mathbf{X} \boldsymbol{\beta})}{\sigma}\right]\right)(\mathbf{y}-\mathbf{X} \boldsymbol{\beta})$.

Intervalos de confiança assintóticos e testes de hipóteses (por exemplo, teste da razão de verossimilhança) para os parâmetros do modelo de regressão normal assimétrico podem ser obtidos usando a matriz $\mathbf{I}(\boldsymbol{\theta})$. Na prática, $\mathbf{I}(\boldsymbol{\theta})$ é substituída pelo EMV $\mathbf{I}(\widehat{\boldsymbol{\theta}})$. A matriz de informação de Fisher esperada é mostrada no Apêndice A.

\subsection{Diagnóstico no Modelo Normal Assimétrico}

Nesta seção serão calculadas a matriz hessiana $\left(\ddot{Q}_{\boldsymbol{\theta}}(\widehat{\boldsymbol{\theta}})\right)$ e as matrizes $\boldsymbol{\Delta}_{\boldsymbol{\omega}_{0}}$ (avaliadas em $\widehat{\boldsymbol{\theta}})$ utilizadas no cálculo da matriz $-\ddot{Q} \boldsymbol{\omega}_{0}=\Delta_{\boldsymbol{\omega}_{o}}^{\top}\left[-\ddot{Q}_{\boldsymbol{\theta}}(\widehat{\boldsymbol{\theta}})\right]^{-1} \boldsymbol{\Delta}_{\boldsymbol{\omega}_{0}}$ para análise de diagnóstico. As perturbações consideradas são: perturbação de ponderações de casos, perturbação no parâmetro de escala $\sigma^{2}$, perturbação no parâmetro de assimetria $\lambda$, perturbação em uma variável explicativa e perturbação na variável resposta. Através desses esquemas de perturbação é possível verificar a sensibilidade de algumas suposições de modelo. Também é calculada a matriz $\ddot{Q}_{\boldsymbol{\theta}, \mathbf{y}}(\widehat{\boldsymbol{\theta}})$ de alavancagem.

Ferreira, C. S. 


\subsubsection{A matriz hessiana}

Para obter as medidas de diagnóstico baseadas na metodologia de Zhu e Lee (2001), é necessário calcular $\ddot{Q}_{\boldsymbol{\theta}}(\widehat{\boldsymbol{\theta}})=\left.\frac{\partial^{2} Q(\boldsymbol{\theta} \mid \widehat{\boldsymbol{\theta}})}{\partial \boldsymbol{\theta} \partial \boldsymbol{\theta}^{\top}}\right|_{\boldsymbol{\theta}=\widehat{\boldsymbol{\theta}}}$, onde $\boldsymbol{\theta}=\left(\boldsymbol{\beta}^{\top}, \sigma^{2}, \lambda\right)^{\top}$. Segue de (2.15) que $\ddot{Q}_{\boldsymbol{\theta}}(\widehat{\boldsymbol{\theta}})$ tem elementos dados por

$$
\begin{aligned}
\frac{\partial^{2} Q(\boldsymbol{\theta} \mid \widehat{\boldsymbol{\theta}})}{\partial \boldsymbol{\beta} \partial \boldsymbol{\beta}^{\top}} & =-\frac{1+\lambda^{2}}{\sigma^{2}} \mathbf{X}^{\top} \mathbf{X}, \\
\frac{\partial^{2} Q(\boldsymbol{\theta} \mid \widehat{\boldsymbol{\theta}})}{\partial \boldsymbol{\beta} \partial \sigma^{2}} & =-\frac{1}{\sigma^{4}} \mathbf{X}^{\top}\left[\left(1+\lambda^{2}\right)(\mathbf{y}-\mathbf{X} \boldsymbol{\beta})-\lambda \widehat{\mathbf{t}}\right], \\
\frac{\partial^{2} Q(\boldsymbol{\theta} \mid \widehat{\boldsymbol{\theta}})}{\partial \boldsymbol{\beta} \partial \lambda} & =\frac{1}{\sigma^{2}} \mathbf{X}^{\top}(2 \lambda(\mathbf{y}-\mathbf{X} \boldsymbol{\beta})-\widehat{\mathbf{t}}), \\
\frac{\partial^{2} Q(\boldsymbol{\theta} \mid \widehat{\boldsymbol{\theta}})}{\partial \sigma^{2} \partial \sigma^{2}} & =\frac{n}{\sigma^{4}}-\frac{1}{\sigma^{6}}\left[\left(1+\lambda^{2}\right) Q(\boldsymbol{\beta})+\widehat{\mathbf{t}}^{\top} \mathbf{1}_{n}-2 \lambda(\mathbf{y}-\mathbf{X} \boldsymbol{\beta})^{\top} \widehat{\mathbf{t}}\right] \\
\frac{\partial^{2} Q(\boldsymbol{\theta} \mid \widehat{\boldsymbol{\theta}})}{\partial \lambda^{2}} & =-\frac{1}{\sigma^{2}} Q(\boldsymbol{\beta}) \mathrm{e} \\
\frac{\partial^{2} Q(\boldsymbol{\theta} \mid \widehat{\boldsymbol{\theta}})}{\partial \sigma^{2} \partial \lambda} & =\frac{1}{\sigma^{4}}\left[\lambda Q(\boldsymbol{\beta})-(\mathbf{y}-\mathbf{X} \boldsymbol{\beta})^{\top} \widehat{\mathbf{t}}\right] .
\end{aligned}
$$

\subsubsection{Perturbação de ponderação de casos}

Esse esquema de perturbação permite identificar aqueles indivíduos que exercem um grande impacto no processo de estimação. Seja $\boldsymbol{\omega}=\left(\omega_{1}, \ldots, \omega_{n}\right)^{\top}$ um vetor de dimensão $n \times 1$ com $\boldsymbol{\omega}_{0}=(1, \ldots, 1)^{\top}$. Então a esperança da função log-verossimilhança de dados completos perturbada (função Q perturbada), pode ser escrita como

$$
Q(\boldsymbol{\theta}, \boldsymbol{\omega} \mid \widehat{\boldsymbol{\theta}})=\mathrm{E}\left[\ell_{c}\left(\boldsymbol{\theta}, \boldsymbol{\omega} \mid \mathbf{y}_{c}\right)\right]=\sum_{i=1}^{n} \omega_{i} \mathrm{E}\left[\ell_{i}\left(\boldsymbol{\theta} \mid \mathbf{y}_{c}\right)\right]=\sum_{i=1}^{n} \omega_{i} Q_{i}(\boldsymbol{\theta} \mid \widehat{\boldsymbol{\theta}}) .
$$

Nesse caso, a matriz $\boldsymbol{\Delta}_{\boldsymbol{\omega}_{0}}=\left.\frac{\partial^{2} Q(\boldsymbol{\theta}, \boldsymbol{\omega} \mid \widehat{\boldsymbol{\theta}})}{\partial \boldsymbol{\theta} \partial \boldsymbol{\omega}^{\top}}\right|_{\boldsymbol{\omega}=\boldsymbol{\omega}_{0}}$ é dada por

$$
\boldsymbol{\Delta}_{\boldsymbol{\omega}_{0}}=\frac{1}{\sigma^{2}}\left[\begin{array}{l}
\left.\mathbf{X}^{\top}\left(\left(1+\lambda^{2}\right) \mathbf{D}(\mathbf{y}-\mathbf{X} \boldsymbol{\beta})-\lambda \mathbf{D} \widehat{\mathbf{t}}\right)\right) \\
\left.-\mathbf{1}_{n}^{\top}+\frac{1}{2 \sigma^{2}}\left[\left(1+\lambda^{2}\right)(\mathbf{y}-\mathbf{X} \boldsymbol{\beta})^{\top} \mathbf{D}(\mathbf{y}-\mathbf{X} \boldsymbol{\beta})+\widehat{\mathbf{t}}^{\top}-2 \lambda(\mathbf{y}-\mathbf{X} \boldsymbol{\beta})^{\top} \mathbf{D} \widehat{\mathbf{t}}\right)\right] \\
(\mathbf{y}-\mathbf{X} \boldsymbol{\beta})^{\top}(\mathbf{D}(\widehat{\mathbf{t}})-\lambda \mathbf{D}(\mathbf{y}-\mathbf{X} \boldsymbol{\beta}))
\end{array}\right] .
$$




\subsubsection{Perturbação do parâmetro de escala}

Esse esquema de perturbação pode revelar indivíduos que interferem principalmente na estrutura de variância do modelo, bem como indicar indivíduos influentes na estimação do parâmetro $\sigma^{2}$. O esquema de perturbação (multiplicativo) se dá na forma

$$
y_{i} \sim N A\left(\mathbf{x}_{i}^{\top} \boldsymbol{\beta}, \omega_{i}^{-1} \sigma^{2}, \lambda\right), \omega_{i}>0, i=1, \ldots, n
$$

A função Q perturbada é da forma

$$
\begin{aligned}
Q(\boldsymbol{\theta}, \boldsymbol{\omega} \mid \widehat{\boldsymbol{\theta}}) \propto & -\sum_{i=1}^{n} \log \frac{\sigma^{2}}{\omega_{i}}-\frac{1}{2 \sigma^{2}} \sum_{i=1}^{n} \omega_{i}{\widehat{t^{2}}}_{i}-\frac{1}{2 \sigma^{2}} \sum_{i=1}^{n} \omega_{i}\left(1+\lambda^{2}\right)\left(y_{i}-\mathbf{x}_{i}^{\top} \boldsymbol{\beta}\right)^{2} \\
& +\frac{\lambda}{\sigma^{2}} \sum_{i=1}^{n} \omega_{i} \widehat{t}_{i}\left(y_{i}-\mathbf{x}_{i}^{\top} \boldsymbol{\beta}\right) .
\end{aligned}
$$

Sob esse esquema de perturbação o modelo não perturbado segue considerando $\boldsymbol{\omega}_{0}=$ $(1, \ldots, 1)^{\top}$ e a matriz $\boldsymbol{\Delta}_{\boldsymbol{\omega}_{0}}$ é dada por

$$
\boldsymbol{\Delta}_{\boldsymbol{\omega}_{0}}=\frac{1}{\sigma^{2}}\left[\begin{array}{l}
\left.\left(1+\lambda^{2}\right) \mathbf{X}^{\top} \mathbf{D}(\mathbf{y}-\mathbf{X} \boldsymbol{\beta})-\lambda \mathbf{X}^{\top} \mathbf{D} \widehat{\mathbf{t}}\right) \\
\frac{1}{2 \sigma^{2}}\left[\left(1+\lambda^{2}\right)(\mathbf{y}-\mathbf{X} \boldsymbol{\beta})^{\top} \mathbf{D}(\mathbf{y}-\mathbf{X} \boldsymbol{\beta})-2 \lambda(\mathbf{y}-\mathbf{X} \boldsymbol{\beta})^{\top} \mathbf{D}(\widehat{\mathbf{t}})+\widehat{\mathbf{t}}^{\top}\right. \\
(\mathbf{y}-\mathbf{X} \boldsymbol{\beta})^{\top}(\mathbf{D}(\widehat{\mathbf{t}})-\lambda \mathbf{D}(\mathbf{y}-\mathbf{X} \boldsymbol{\beta}))
\end{array}\right] .
$$

\subsubsection{Perturbação do parâmetro de assimetria}

Esse esquema de perturbação pode revelar indivíduos que interferem principalmente na estrutura de variância do modelo, bem como indicar indivíduos influentes na estimação do parâmetro $\lambda$. O esquema de perturbação (multiplicativo) se dá na forma

$$
y_{i} \sim N A\left(\mathbf{x}_{i}^{\top} \boldsymbol{\beta}, \sigma^{2}, \lambda \omega_{i}\right), \omega_{i} \in \mathbb{R}, i=1, \ldots, n
$$

Sendo $\boldsymbol{\omega}=\left(\omega_{1}, \ldots, \omega_{n}\right)^{\top}$, a função $Q$ perturbada é da forma

$$
\begin{aligned}
Q(\boldsymbol{\theta}, \boldsymbol{\omega} \mid \widehat{\boldsymbol{\theta}}) \propto & -n \log \sigma^{2}-\frac{1}{2 \sigma^{2}} \sum_{i=1}^{n}{\widehat{t^{2}}}_{i}-\frac{1}{2 \sigma^{2}} \sum_{i=1}^{n}\left(1+\lambda^{2} \omega_{i}^{2}\right)\left(y_{i}-\mathbf{x}_{i}^{\top} \boldsymbol{\beta}\right)^{2} \\
& +\frac{\lambda}{\sigma^{2}} \sum_{i=1}^{n} \omega_{i} \widehat{t}_{i}\left(y_{i}-\mathbf{x}_{i}^{\top} \boldsymbol{\beta}\right) .
\end{aligned}
$$

Ferreira, C. S. 
Sob esse esquema de perturbação o modelo não perturbado segue considerando $\boldsymbol{\omega}_{0}=$ $(1, \ldots, 1)^{\top}$ e a matriz $\boldsymbol{\Delta}_{\boldsymbol{\omega}_{0}}$ é dada por

$$
\boldsymbol{\Delta}_{\boldsymbol{\omega}_{0}}=\frac{1}{\sigma^{2}}\left[\begin{array}{l}
\lambda \mathbf{X}^{\top}(2 \lambda \mathbf{D}(\mathbf{y}-\mathbf{X} \boldsymbol{\beta})-\mathbf{D}(\widehat{\mathbf{t}})) \\
\frac{\lambda}{\sigma^{2}}(\mathbf{y}-\mathbf{X} \boldsymbol{\beta})^{\top}[\lambda \mathbf{D}(\mathbf{y}-\mathbf{X} \boldsymbol{\beta})-\mathbf{D}(\widehat{\mathbf{t}})] \\
(\mathbf{y}-\mathbf{X} \boldsymbol{\beta})^{\top}(\mathbf{D}(\widehat{\mathbf{t}})-2 \lambda \mathbf{D}(\mathbf{y}-\mathbf{X} \boldsymbol{\beta}))
\end{array}\right] .
$$

\subsubsection{Perturbação em uma variável explicativa}

Nesse caso o interesse está em perturbar uma variável explicativa específica permitindo, por exemplo, detectar possíveis maus condicionamentos de alguma coluna da matriz de desenho (Belsley, 1991). Sob essa condição, tem-se a seguinte o esquema de perturbação aditiva em uma variável explicativa $\left(X_{v}\right)$

$$
x_{i v}(\boldsymbol{\omega})=x_{i v}+S_{v} \omega_{i}, \quad v \in\{1, \ldots, p\}, \omega_{i} \in \mathbb{R}, i=1, \ldots, n,
$$

onde $S_{v}$ é o desvio padrão da variável explicativa $X_{v}$. Nesse caso, $\boldsymbol{\omega}_{0}=\mathbf{0}$ e a função $\mathrm{Q}$ perturbada é dada por

$$
Q(\boldsymbol{\theta}, \boldsymbol{\omega} \mid \widehat{\boldsymbol{\theta}}) \propto-n \log \sigma^{2}-\frac{1+\lambda^{2}}{2 \sigma^{2}}\left(\mathbf{y}-\mathbf{X}_{\boldsymbol{\omega}} \boldsymbol{\beta}\right)^{\top}\left(\mathbf{y}-\mathbf{X}_{\boldsymbol{\omega}} \boldsymbol{\beta}\right)-\frac{1}{2 \sigma^{2}} \widehat{\mathbf{t}}^{\top} \mathbf{1}_{n}+\frac{\lambda}{\sigma^{2}}\left(\mathbf{y}-\mathbf{X}_{\boldsymbol{\omega}} \boldsymbol{\beta}\right)^{\top} \widehat{\mathbf{t}},
$$

onde $\mathbf{X}_{\boldsymbol{\omega}}=\mathbf{X}+S_{v} \mathbf{A}_{v}$, onde $\mathbf{A}_{v}$ é uma matriz de zeros com a $v$-ésima coluna de 1's.

Segue que

$$
\boldsymbol{\Delta} \boldsymbol{\omega}_{0}=\frac{S_{v}}{\sigma^{2}}\left[\begin{array}{l}
\left(1+\lambda^{2}\right)\left(\mathbf{0}_{v}^{1}(\mathbf{y}-\mathbf{X} \boldsymbol{\beta})^{\top}-\beta_{v} \mathbf{X}^{\top}\right)-\lambda \mathbf{0}_{v}^{1} \widehat{\mathbf{t}}^{\top} \\
\frac{\beta_{v}}{\sigma^{2}}\left(\left(1+\lambda^{2}\right)(\mathbf{y}-\mathbf{X} \boldsymbol{\beta})^{\top}+\lambda \widehat{\mathbf{t}}^{\top}\right) \\
\beta_{v}\left(-\widehat{\mathbf{t}}^{\top}+2 \lambda(\mathbf{y}-\mathbf{X} \boldsymbol{\beta})^{\top}\right)
\end{array}\right]
$$

onde $\mathbf{0}_{v}^{1}$ denota um vetor de zeros de tamanho $p \times 1$ com um 1 na $v$-ésima posição.

\subsubsection{Perturbação na variável resposta}

Este esquema de perturbação permite verificar mau condicionamento na variável resposta. Uma característica de interesse deste esquema de perturbação é a sua conexão com a matriz de alavancas generalizadas (Wei et al., 1998). A perturbação em $\mathbf{y}=\left(y_{1}, \ldots, y_{n}\right)^{\top}$ é 
introduzida substituindo $y_{i}$ por $y_{i}(\boldsymbol{\omega})=y_{i}+\omega_{i} S_{y}, i=1, \ldots, n$., onde $S_{y}$ é o desvio padrão de $\mathbf{y}$. Neste caso $\boldsymbol{\omega}_{0}=\mathbf{0}$ e

$$
Q(\boldsymbol{\theta}, \boldsymbol{\omega} \mid \widehat{\boldsymbol{\theta}}) \propto-n \log \sigma^{2}-\frac{1+\lambda^{2}}{2 \sigma^{2}}(\mathbf{y} \boldsymbol{\omega}-\mathbf{X} \boldsymbol{\beta})^{\top}(\mathbf{y} \boldsymbol{\omega}-\mathbf{X} \boldsymbol{\beta})-\frac{1}{2 \sigma^{2}} \widehat{\mathbf{t}}^{\top} \mathbf{1}_{n}+\frac{\lambda}{\sigma^{2}}(\mathbf{y} \boldsymbol{\omega}-\mathbf{X} \boldsymbol{\beta})^{\top} \widehat{\mathbf{t}},
$$

onde $\mathbf{y} \boldsymbol{\omega}=\mathbf{y}+S_{y} \boldsymbol{\omega}$.

Segue que

$$
\boldsymbol{\Delta} \boldsymbol{\omega}_{0}=\frac{S_{y}}{\sigma^{2}}\left[\begin{array}{l}
\left(1+\lambda^{2}\right) \mathbf{X}^{\top} \\
\frac{1}{\sigma^{2}}\left(\left(1+\lambda^{2}\right)(\mathbf{y}-\mathbf{X} \boldsymbol{\beta})^{\top}-\lambda \widehat{\mathbf{t}}^{\top}\right) \\
\widehat{\mathbf{t}}^{\top}-2 \lambda(\mathbf{y}-\mathbf{X} \boldsymbol{\beta})^{\top}
\end{array}\right]
$$

\subsubsection{Alavancagem generalizada}

Para o modelo normal assimétrico, tem-se que $\boldsymbol{\mu}=E[\mathbf{Y}]=\mathbf{X} \boldsymbol{\beta}+c \sigma \rho \mathbf{1}_{n}$, onde $c=\sqrt{2 / \pi}$ e $\rho=\frac{\lambda}{\sqrt{1+\lambda^{2}}}$. Deste modo, a matriz $D_{\boldsymbol{\theta}}: n \times(p+2)$ é da forma

$$
D_{\boldsymbol{\theta}}=\left[\mathbf{X}, \frac{c \rho}{2 \sigma} \mathbf{1}_{n}, \frac{c \sigma}{\left(1+\lambda^{2}\right)^{3 / 2}} \mathbf{1}_{n}\right]
$$

A matriz $\ddot{Q}_{\boldsymbol{\theta}, \mathbf{y}}(\widehat{\boldsymbol{\theta}})$ é como em (2.20) sem o termo $S_{y}$ e $\ddot{Q}_{\boldsymbol{\theta}}(\widehat{\boldsymbol{\theta}})$ é dada na Seção 2.5.1.

\subsection{Estudo de Dados Artificiais}

Para verificar a validade do algoritmo EM proposto e análise de diagnóstico, foram simulados dados de um modelo com uma variável regressora, da forma $y_{i}=\alpha+\beta x_{i}+\varepsilon_{i}$, onde $\varepsilon_{i} \sim N A\left(0, \sigma^{2}, \lambda\right), i=1, \ldots, 200$ e a variável $x$ foi gerada da distribuição $U(0,1)$. Os valores escolhidos dos parâmetros foram $\alpha=1, \beta=2, \sigma^{2}=0.5$ e $\lambda=3$. A Tabela 2.1 apresenta os valores escolhidos e estimados dos parâmetros do modelo. Os erros padrão são calculados utilizando a matriz de informação de Fisher observada, na forma $\operatorname{EP}(\widehat{\boldsymbol{\theta}})=\sqrt{\mathbf{D}\left(I^{-1}(\widehat{\boldsymbol{\theta}})\right)}$.

O gráfico de envelope simulado, utilizando a representação dada em (2.11), não acusa pontos além da banda de confiança (Fig 2.2).

A análise de diagnóstico revelou que os pontos extremos abaixo da reta estimada são influentes sob perturbações de ponderação de casos, perturbação na escala, perturbação na 


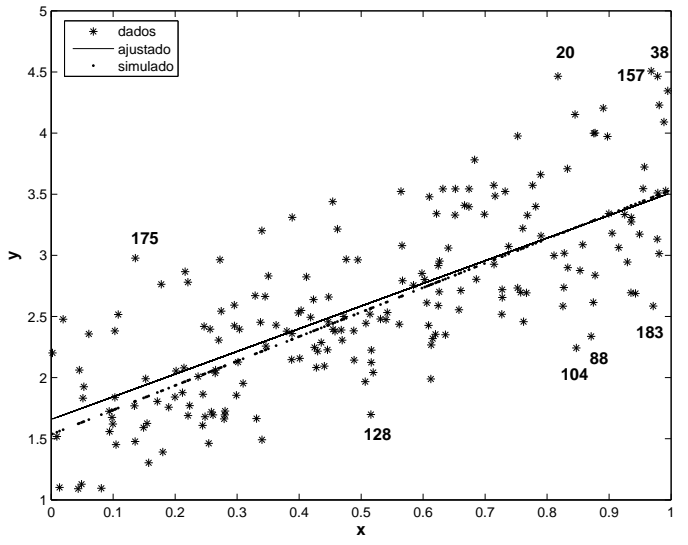

(a)

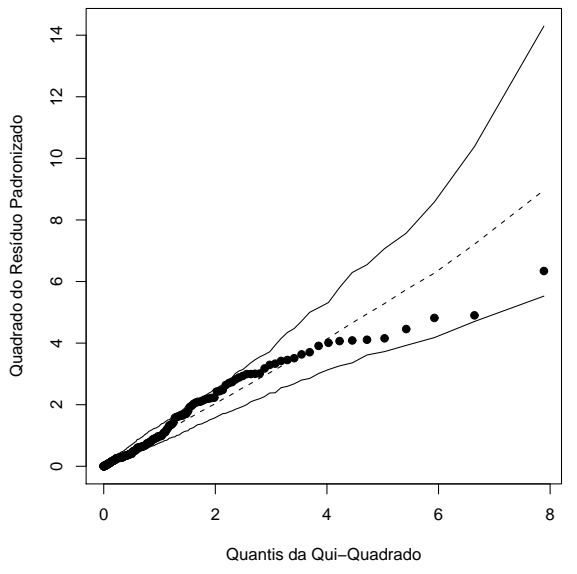

(b)

Figura 2.2: Modelo de regressão com dados simulados. (a) Gráfico de dispersão, juntamente com as retas do modelo normal assimétrico simulado e ajustado e pontos influentes e (b) gráfico de envelope simulado.

Tabela 2.1: Modelo de regressão NA com dados simulados: Estimativas dos parâmetros do modelo, com seus respectivos erros padrão assintóticos entre parênteses.

\begin{tabular}{l|ccccc}
\hline \hline & $\alpha$ & $\beta$ & $\sigma^{2}$ & $\lambda$ & $l(\boldsymbol{\theta})$ \\
\hline Valores verdadeiros & 1 & 2 & 0.5 & 3 & - \\
Estimativas & $1.10(0.07)$ & $1.85(0.11)$ & $0.53(0.08)$ & $2.94(0.73)$ & -129.41 \\
\hline \hline
\end{tabular}

variável resposta e na variável explicativa (esses dois últimos muito semelhantes). Por outro lado, são os pontos extremos acima da reta estimada os influentes sob a perturbação em $\lambda$.

A análise de influência global mostrou que a medida $L D_{i}^{c}$ é muito sensível a pontos extremos abaixo e acima da reta estimada. Por outro lado, o gráfico para $D_{i}^{c}$ identificou pontos influentes localizados abaixo da reta.

Ferreira, C. S. 


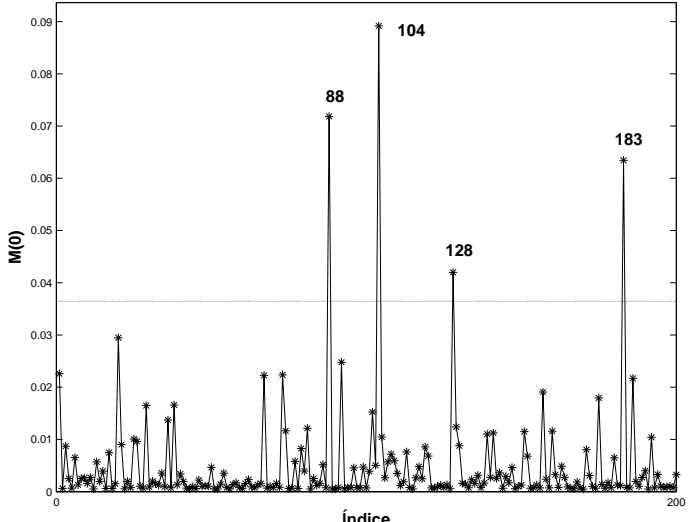

(a)

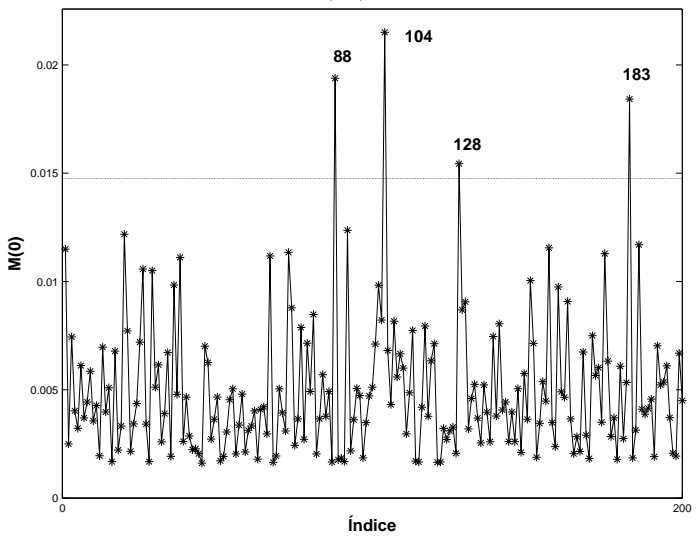

(c)

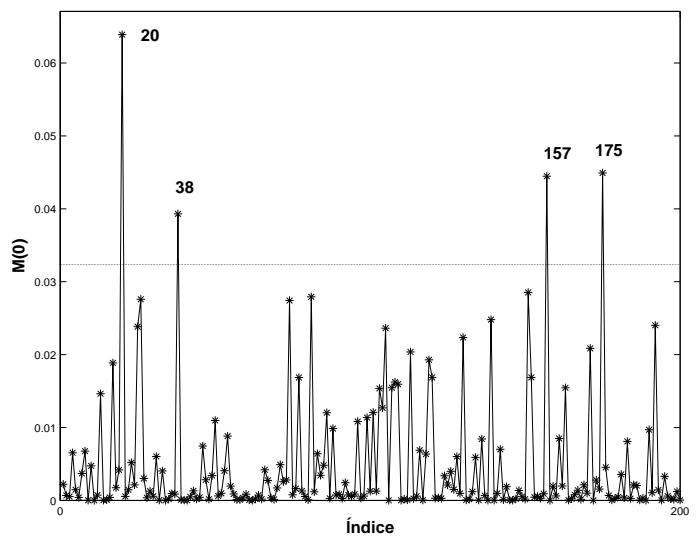

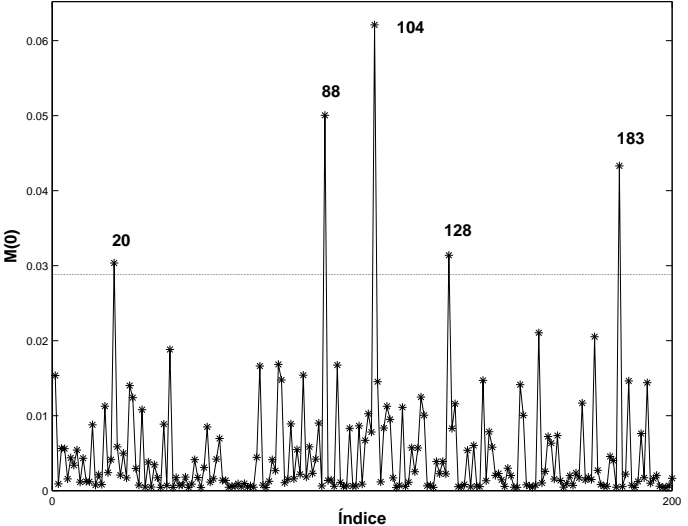

(b)

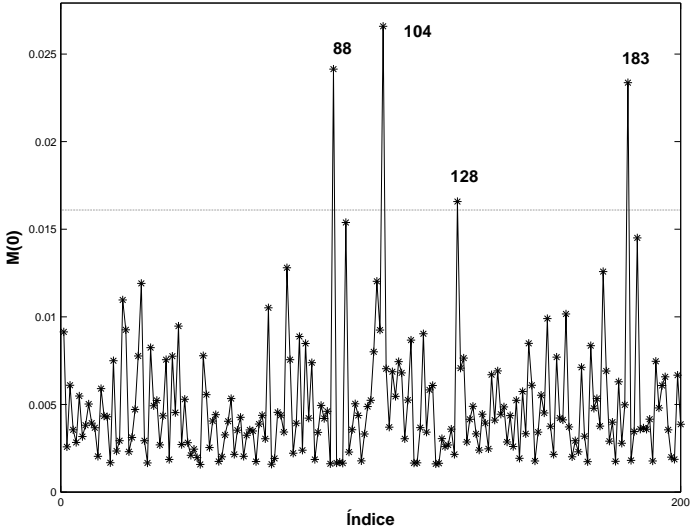

(d)

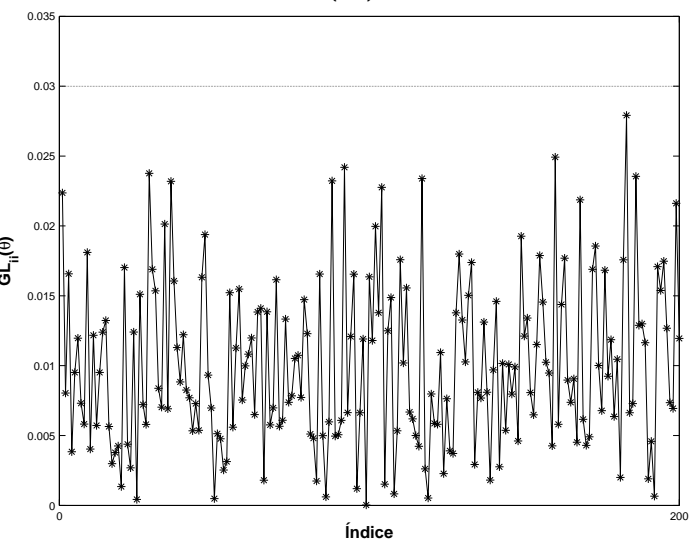

e)

Figura 2.3: Diagnóstico para o modelo de regressão com dados simulados. Linhas horizontais são os pontos de corte $\left(c^{*}=3\right)$. (a) Perturbação de ponderação de casos, (b) perturbação na escala, (c) perturbação na variável resposta, (d) perturbação na variável explicativa, (e) perturbação em $\lambda$ e (f) alavanca. 


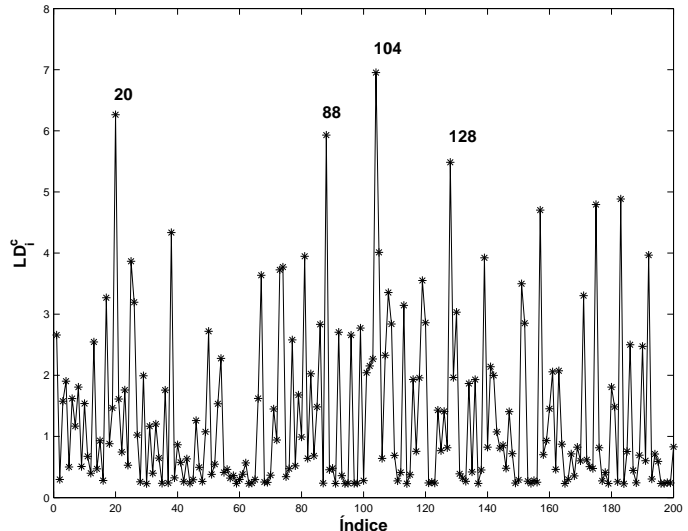

(a)

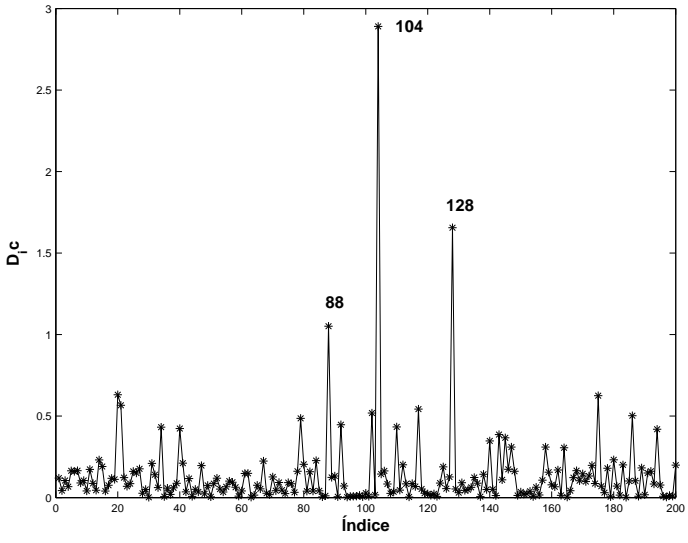

(b)

Figura 2.4: Influência global para o modelo de regressão com dados simulados. (a) $\left(L D_{i}^{c}\right)$ e (b) $\left(D_{i}^{c}\right)$

\subsection{Aplicação}

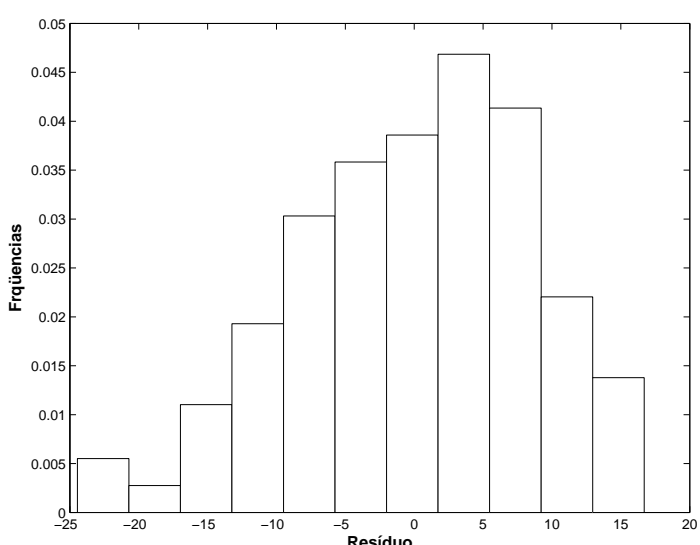

(a)

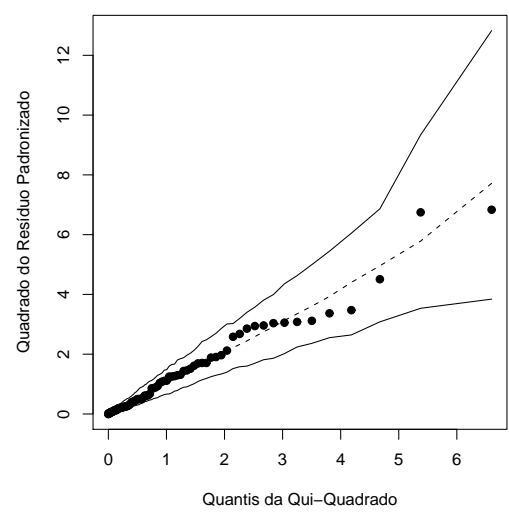

(b)

Figura 2.5: Ajuste do modelo normal assimétrico para o conjunto de dados sobre qualidade de vida. (a) histograma do resíduo do modelo e (b) gráfico de envelope simulado.

Para ilustrar a aplicação do modelo de regressão normal assimétrico e a análise de diagnóstico, foi ajustado um modelo a um conjunto de dados sobre qualidade de vida de pacientes (mulheres) com câncer de mama, em um estudo realizado pelo Centro de Atenção Integral à Saúde da Mulher (CAISM) em conjunto com a Faculdade de Ciências Médicas, da Universidade Estadual de Campinas. O índice de qualidade de vida é obtido através de um questionário de 36 ítens (SF-36), amplamente utilizado em pesquisas da área de saúde. 
O mesmo é dividido em duas componentes, uma física e uma mental. Conde et al. (2005) estudaram esse conjunto de dados, avaliando os fatores associados à qualidade de vida de mulheres com câncer de mama. As estatísticas descritivas para o índice de qualidade de vida (componente física, pcs, em inglês, physical component summary) e para o índice de massa corpórea $(\mathrm{imc})$ do indivíduo são apresentadas na Tabela 2.2. A variável indicadora tontura $(1=$ sente tontura, $0=$ não sente tontura $)$ apresenta uma proporção de $34 \%$ de indivíduos que sentem tontura.

Tabela 2.2: Conjunto de dados sobre qualidade de vida: Estatísticas descritivas para o índice de massa corpórea (imc) e componente física do índice de qualidade de vida (pcs) dos indivíduos (DP é o desvio-padrão).

\begin{tabular}{c|ccccccc}
\hline \hline & Mínimo & Média & Máximo & Percentil 25 & Mediana & Percentil 75 & DP \\
\hline imc & 19.64 & 28.00 & 50.90 & 24.28 & 27.79 & 30.35 & 5.51 \\
pcs & 20.90 & 46.54 & 64.70 & 37.65 & 50.00 & 53.83 & 9.86 \\
\hline \hline
\end{tabular}

O modelo de regressão foi construído utilizando como variável dependente o índice de qualidade de vida e como variáveis regressoras a variável indicadora tontura e o índice de massa corpórea do indivíduo, levando ao modelo

$$
\begin{aligned}
p c s_{i} & =\beta_{0}+\beta_{1} \times \text { tontura }_{i}+\beta_{2} \times i m c_{i}+\varepsilon_{i}, \\
\varepsilon_{i} & \sim N A\left(0, \sigma^{2}, \lambda\right), \quad i=1, \ldots, 97 .
\end{aligned}
$$

Ajustado o modelo, os parâmetros estimados, juntamente com seus respectivos erros padrão estimados são descritos na Tabela 2.3. Os erros padrão são calculados utilizando a matriz de informação de Fisher observada, na forma $E P(\widehat{\boldsymbol{\theta}})=\sqrt{\mathbf{D}\left(I^{-1}(\widehat{\boldsymbol{\theta}})\right)}$. O resíduo é calculado como $y_{i}-\mathbf{x}_{i}^{\top} \widehat{\boldsymbol{\beta}}-\sqrt{\frac{2}{\pi}} \frac{\widehat{\sigma} \widehat{\lambda}}{1+\widehat{\lambda}^{2}}$.

Tabela 2.3: EMVs do modelo normal e modelo normal assimétrico (NA) para o conjunto de dados sobre qualidade de vida (erro padrão estimado assintótico entre parênteses)

\begin{tabular}{lcccccc}
\hline \hline Modelo & $\widehat{\beta}_{0}$ & $\widehat{\beta}_{1}$ & $\widehat{\beta}_{2}$ & $\widehat{\sigma^{2}}$ & $\widehat{\lambda}$ & $\ell(\widehat{\boldsymbol{\theta}})$ \\
\hline NA & $68.57(4.64)$ & $-7.62(1.91)$ & $-0.35(0.17)$ & $169.41(45.07)$ & $-2.38(1.09)$ & -346.20 \\
Normal & $61.29(4.75)$ & $-7.92(1.91)$ & $-0.43(0.16)$ & $79.13(11.73)$ & - & -348.14 \\
\hline \hline
\end{tabular}

Ferreira, C. S. 


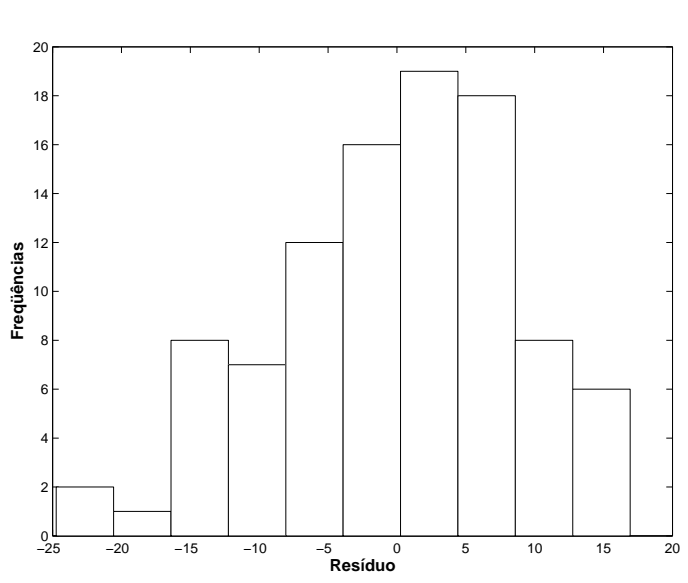

(a)

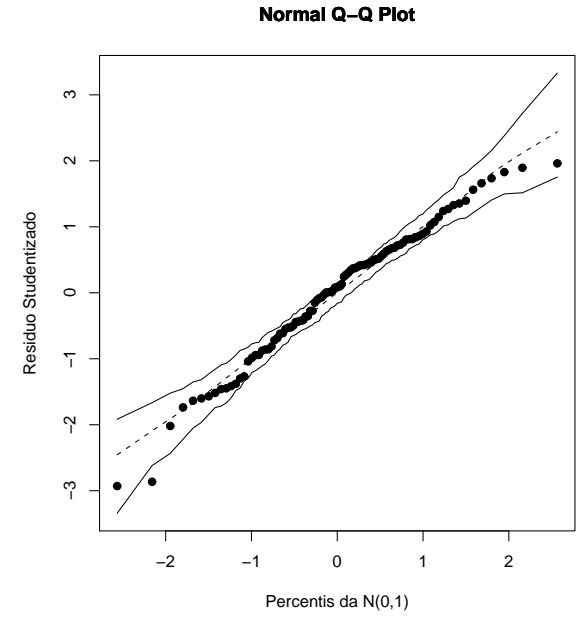

(b)

Figura 2.6: Ajuste do modelo de regressão múltipla (normal) para o conjunto de dados sobre qualidade de vida. (a) histograma do resíduo do modelo e (b) gráfico de envelope simulado.

Para verificar a validade do modelo, foi construído o gráfico de envelope simulado, utilizando a representação dada em (2.11), não acusando pontos além da banda de confiança (Fig 2.5). Por outro lado, o gráfico de envelope para o modelo normal (Figura 2.6) indica seis pontos além da banda de confiança.

Comparando com o modelo normal (Tabela 2.3), as estimativas de $\boldsymbol{\beta}$ nos dois modelos são parecidas. Por outro lado, as estimativas do parâmetros de escala $\sigma^{2}$ são diferentes. Realizando um teste de hipóteses assintótico (teste de razão de verossimilhança) para testar $H_{0}: \lambda=0 \times H_{1}: \lambda \neq 0$, tem-se que $R V=2\left(\ell(\widehat{\boldsymbol{\theta}})-\ell\left(\widehat{\boldsymbol{\theta}}_{0}\right)\right)=3.88$, com um p-valor de 0.05, levando à rejeição da hipótese nula. Ou seja, um modelo normal assimétrico é mais apropriado que o modelo de regressão múltipla usual, assumindo erros normais.

A análise de diagnóstico através de influência local detectou pontos potencialmente influentes. Os pontos 28 e 52 (mulheres com imc médio e alto índice de qualidade de vida, sentindo tontura) são influentes sob perturbação de ponderação de casos. Esses dois pontos, adicionais aos pontos 42 e 73 (mulheres com baixos $i m c$ e $p c s$, não sentindo tontura) são influentes sob perturbação na escala. Ainda, os pontos 28 e 52, adicionados ao ponto 65 (mulher com médio $i m c$ e alto índice $p c s$, não sentindo tontura) são pontos de alavanca.

Os pontos 15 e 23 (mulheres com alto imc e pcs abaixo da média, não sentindo tontura) são influentes sob perturbação na variável resposta pcs e na variável explicativa imc. Por 
último, os pontos 23, 42 e 73 (mulheres com baixos $i m c$ e $p c s$, não sentindo tontura) são influentes sob perturbação em $\lambda$.

A análise de influência global, tanto $L D_{i}^{c}$ como $D_{i}^{c}$, indicou o ponto 20 , uma mulher com baixos imc e pcs, sentindo tontura.

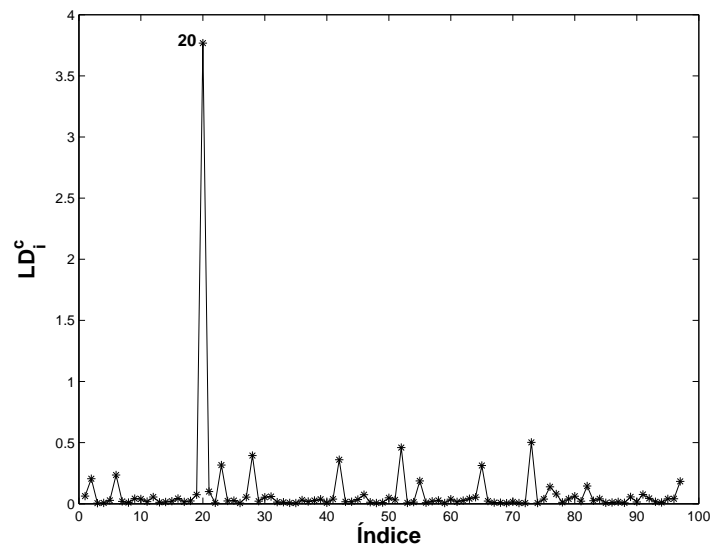

(a)

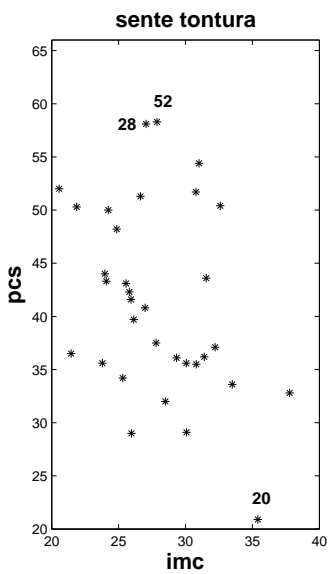

(c)

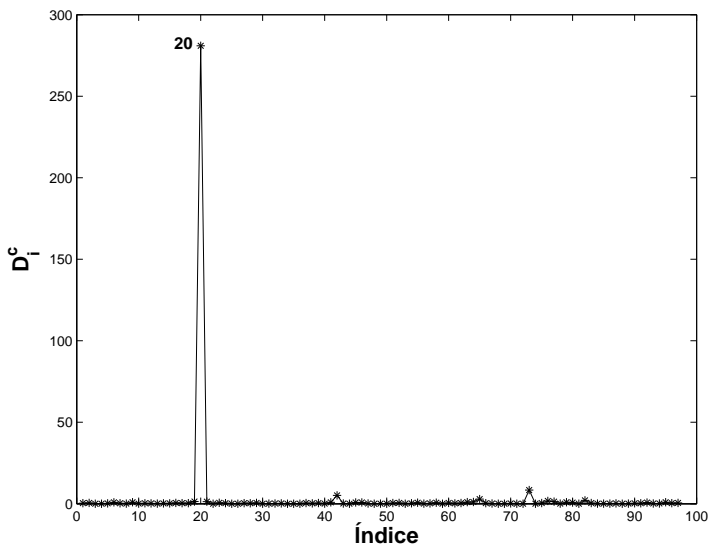

(b)

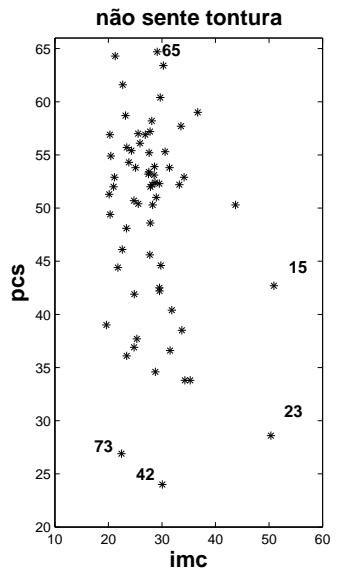

Figura 2.7: Diagnóstico para o conjunto de dados de qualidade de vida. (a) influência global $\left(L D_{i}^{c}\right)$, (b) influência global $\left(D_{i}^{c}\right)$ e $($ c) gráfico de dispersão imc $\times p c s$, por tontura, com pontos influentes.

Ferreira, C. S. 


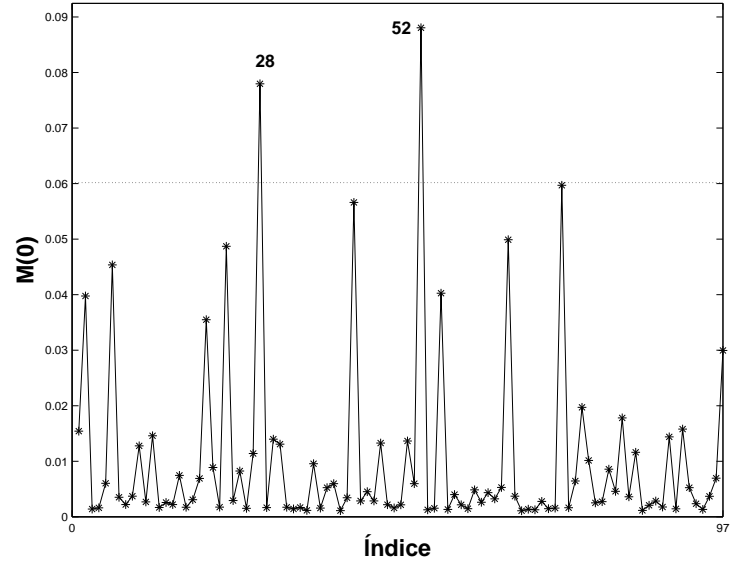

(a)

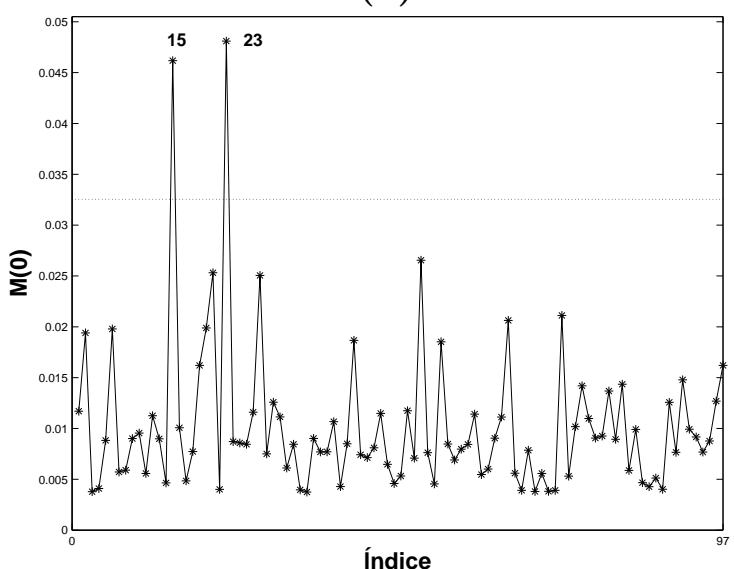

(c)

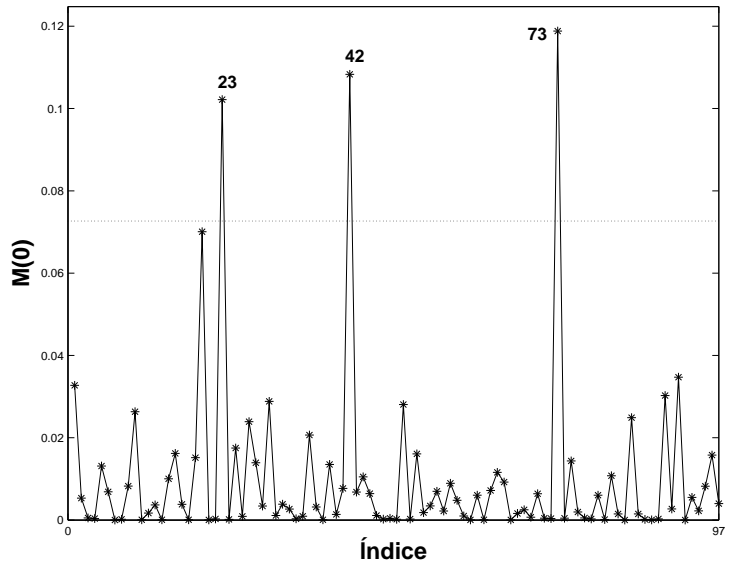

(e)

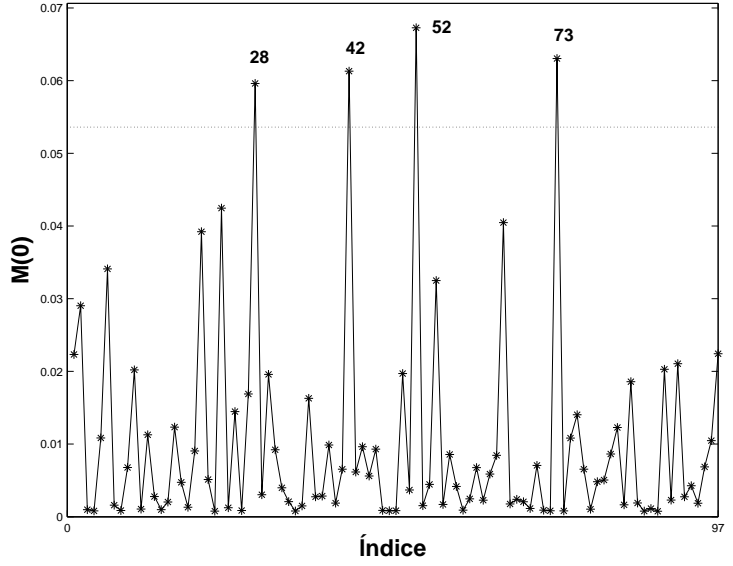

(b)

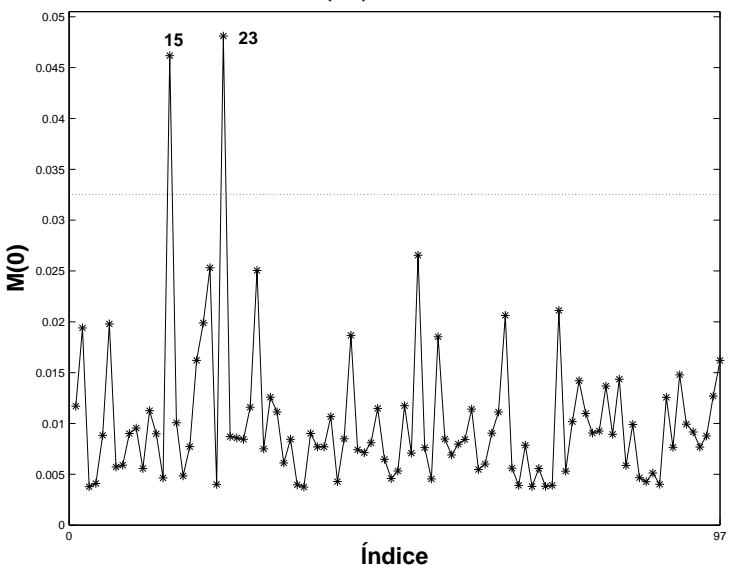

(d)

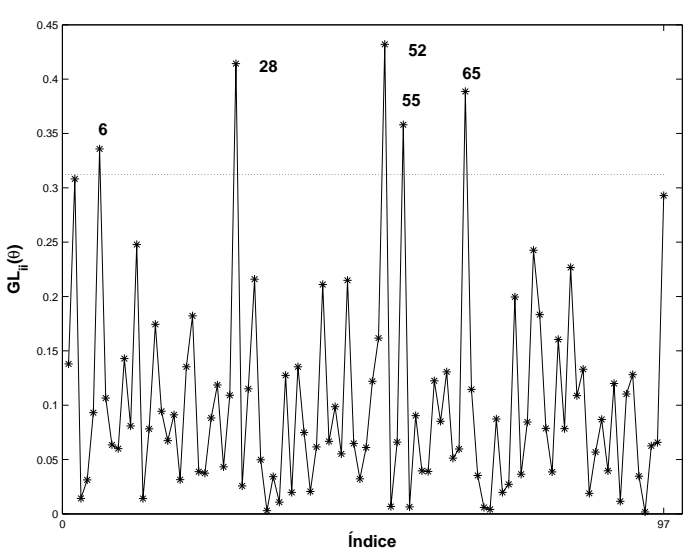

(f)

Figura 2.8: Diagnóstico para o conjunto de dados de qualidade de vida. Linhas horizontais são os pontos de corte $\left(c^{*}=3\right)$. (a) Perturbação de ponderação de casos, (b) perturbação na escala, (c) perturbação na variável resposta, (d) perturbação na variável explicativa, (e) perturbação em $\lambda$ e (f) alavanca. 


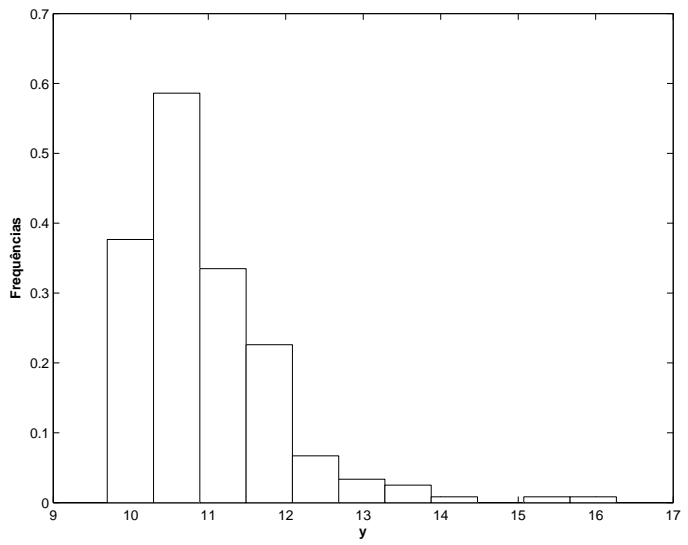

(a)

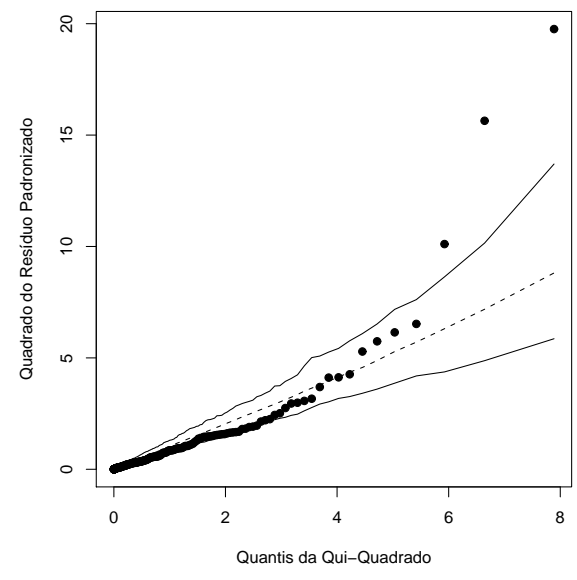

(b)

Figura 2.9: Dados simulados da $N A(10,1,5), n=200$, com pontos perturbados na cauda pesada da amostra. (a) Histograma e (b) envelope simulado.

\subsection{Falta de Ajuste no Modelo Normal Assimétrico}

Embora o modelo normal assimétrico, introduzido por Azzalini (1985, 1986), tenha sido uma grande contribuição na análise de dados com estruturas assimétricas, ele não se comporta bem na presença de observações extremas na cauda pesada. Para mostrar essa deficiência, simulamos uma amostra de tamanho 100 de $Y \sim N A(10,1,5)$ e perturbamos observações de tal forma a torná-las extremas (na cauda pesada da amostra), na seguinte forma $y_{(k)}=1.05 * y(n), k=98,99,100$, onde $y_{(k)}$ é a estatística de ordem $k$ do vetor $\mathbf{y}$. O histograma desses dados encontra-se na Fig. 2.9. O gráfico de envelope na mesma figura mostra a falta de ajuste do modelo normal assimétrico.

Faz-se então necessário buscar um modelo assimétrico mais robusto, que incorpore a assimetria dos dados e ainda capture a presença de observações extremas. O modelo tnormal assimétrico, introduzido por Gómez, Venegas e Bolfarine (2007), que será visto no próximo capítulo, dá conta desse problema de assimetria com caudas mais pesadas que o modelo normal assimétrico de Azzalini. 


\section{Modelo t-Normal Assimétrico}

\subsection{Introdução}

A distribuição t assimétrica tem sido um tópico de interesse crescente na literatura estatística, sendo estudada por Branco e Dey (2001), Azzalini e Capitanio (2003), Sahu (2003), Nadarajah e Kotz (2003), entre outros. Gupta (2003) propôs a distribuição t assimétrica e estudou muitas de suas propriedades. Recentemente, Gómez, Venegas e Bolfarine (2007) introduziram a distribuição t-normal assimétrica e mostraram propriedades interessantes dessa distribuição. Os intervalos de assimetria e curtose são mais amplos que os introduzidos por Nadarajah e Kotz (2003) e a matriz de informação de Fisher é sempre não-singular para valores finitos dos graus de liberdade. Sabe-se que, no caso da distribuição normal assimétrica, a matriz de informação de Fisher pode ser singular (por exemplo, em $\lambda=0$ ) (Azzalini, 1985).

Segundo Gómez, Venegas e Bolfarine (2007), diz-se que uma variável aleatória $Y$ segue uma distribuição t-normal assimétrica com parâmetro de locação $\mu$, parâmetro de escala $\sigma^{2}$ e parâmetro de assimetria $\lambda$ se sua função densidade de probabilidade (fdp) é dada por

$$
f(y)=2 t_{\nu}\left(y \mid \mu, \sigma^{2}\right) \Phi\left(\lambda \frac{y-\mu}{\sigma}\right), \quad y \in \mathbb{R}
$$

onde $\Phi($.$) denota a função de distribuição acumulada (fda) de uma distribuição N(0,1)$ e $t_{\nu}(. \mid a, b)$ denota a fdp de uma distribuição t de Student com parâmetro de locação $a$, parâmetro de escala $b$ e $\nu$ graus de liberdade $\left(t_{\nu}(a, b)\right)$. Será utilizada a notação $Y \sim$ $T N A_{\nu}\left(\mu, \sigma^{2}, \lambda\right)$ para denotar a distribuição seguida por $Y$ e a notação $Y \sim T N A_{\nu}(\lambda)$ quando $\mu=0$ e $\sigma^{2}=1$. Se $\lambda=0$, obtém-se a densidade ordinária t de Student com $\nu$ graus de liberdade. 
De acordo com Gómez et al. (2007), os momentos de ordem $k$ (par) da distribuição t-normal assimétrica padrão $Y \sim T N A_{\nu}(\lambda)$ para $\nu>k$ são dados por

$$
E\left[Y^{k}\right]=\frac{\nu^{k / 2} \Gamma\left(\frac{k+1}{2}\right) \Gamma\left(\frac{\nu-k}{2}\right)}{\sqrt{\pi} \Gamma\left(\frac{\nu}{2}\right)} .
$$

Assim, tem-se que

$$
E\left[Y^{2}\right]=\frac{\nu}{\nu-2} \quad \text { e } \quad E\left[Y^{4}\right]=\frac{3 \nu^{2}}{(\nu-4)(\nu-2)}, \quad \nu>4
$$

Para $k$ ímpar e $\nu$ par, os momentos de ordem $k$ são dados por

$$
\begin{aligned}
E\left[Y^{k}\right]= & \frac{\sqrt{2} \lambda \nu^{(k+1) / 2} \Gamma\left(\frac{\nu-1-k}{2}\right) \Gamma\left(\frac{k+2}{2}\right)}{\pi \Gamma\left(\frac{\nu}{2}\right)}{ }_{2} F_{2}\left(\frac{1}{2}, \frac{2+k}{2} ; \frac{3}{2}, \frac{3-\nu+k}{2} ; \frac{\nu \lambda^{2}}{2}\right) \\
& +\frac{2^{\frac{k-\nu+4}{2}} \nu^{\nu / 2}\left[|\lambda|^{\nu-k} \operatorname{sinal} \lambda\right] \Gamma\left(\frac{\nu+1}{2}\right)}{(\nu-k)(\nu-k-1) \cos \left[\frac{(k-\nu) \pi}{2}\right] \Gamma\left(\frac{\nu}{2}\right) \Gamma\left(\frac{(\nu-k-1)}{2}\right)} \\
\times & { }_{2} F_{2}\left(\frac{\nu+1}{2}, \frac{\nu-k}{2} ; \frac{1+\nu-k}{2}, \frac{2+\nu-k}{2} ; \frac{\nu \lambda^{2}}{2}\right),
\end{aligned}
$$

onde

$$
{ }_{2} F_{2}(\mathbf{n} ; \mathbf{d} ; z)=\sum_{r=0}^{\infty}\left(\prod_{i=1}^{2} \frac{\Gamma\left(n_{i}+r\right)}{\Gamma\left(n_{i}\right) z^{r}} / \prod_{i=1}^{2} \frac{\Gamma\left(d_{i}+r\right)}{\Gamma\left(d_{i}\right) r !}\right),
$$

$\mathbf{n}=\left[n_{1}, n_{2}\right]$ e $\mathbf{d}=\left[d_{1}, d_{2}\right]$ (função hipergeométrica generalizada). Por último, para $k$ e $\nu$ ímpares, os momentos têm de ser calculados numericamente.

Também é verdade que

$$
D=\frac{(Y-\mu)^{2}}{\sigma^{2}} \sim F(1, \nu),
$$

onde $F(m, n)$ denota a distribuição $F$ de Snedecor-Fisher com graus de liberdade $m$ e $n$. Esse resultado permite checar o modelo na prática. Gómez et al. (2007) mostram que a distribuição t-normal assimétrica pode apresentar um intervalo de valores do coeficiente de assimetria muito mais amplo que a distribuição normal assimétrica. Ainda, os autores apresentam um procedimento iterativo para estimação de máxima verossimilhança (EMV) baseado na função log-verossimilhança marginal de $Y$. Diferentemente, propõe-se neste trabalho um algoritmo tipo EM para obter as estimativas de máxima verossimilhança dos parâmetros de um modelo de regressão linear t-normal assimétrico independente, que é mais robusto com respeito aos valores iniciais.

Ferreira, C. S. 


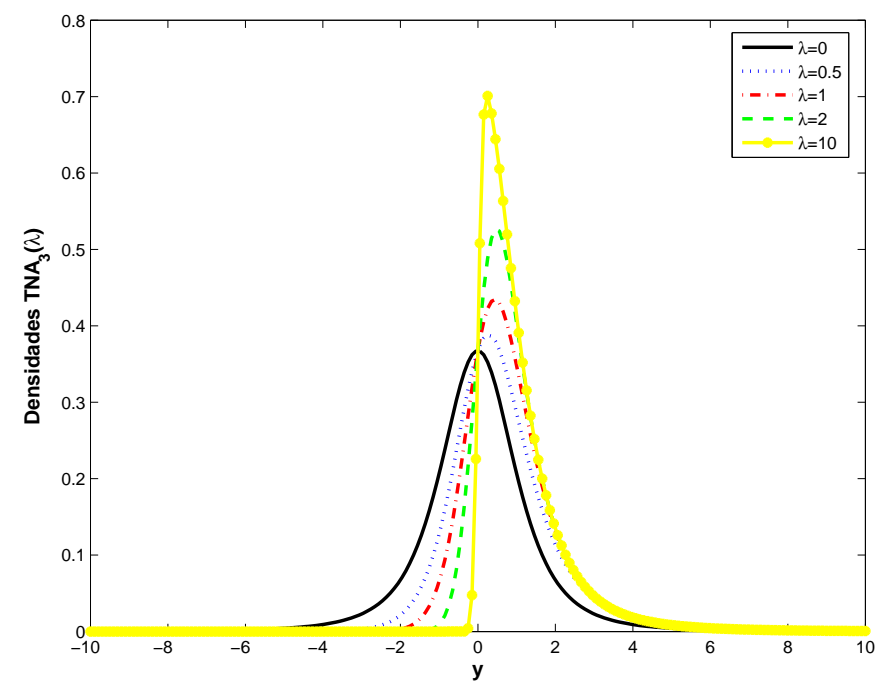

(a)

Figura 3.1: Densidades $T N A_{3}(\lambda)$.

\subsection{Modelo de Regressão t-Normal Assimétrico e Al- goritmo EM}

Suponha que temos observações de $n$ indivíduos independentes, $Y_{1}, \ldots, Y_{n}$, onde $Y_{i} \sim$ $T N A_{\nu}\left(\mu_{i}, \sigma^{2}, \lambda\right), i=1, \ldots, n$. Associado ao indivíduo $i$ assume-se um vetor de covariáveis $p \times 1$ conhecido $\mathbf{x}_{i}$, que será utilizado para especificar o preditor linear $\mu_{i}=\mathbf{x}_{i}^{\top} \boldsymbol{\beta}$, onde $\boldsymbol{\beta}$ é um vetor $p$-dimensional de coeficientes desconhecidos de regressão. Sob essas condições, a função log-verossimilhança de $\boldsymbol{\theta}=\left(\boldsymbol{\beta}^{\top}, \sigma^{2}, \lambda\right)^{\top}$ é dada por

$$
\ell(\boldsymbol{\theta})=n \log c(\nu)-\frac{n}{2} \log \sigma^{2}-\frac{\nu+1}{2} \sum_{i=1}^{n} \log \left[1+\frac{\left(y_{i}-\mathbf{x}_{i}^{\top} \boldsymbol{\beta}\right)^{2}}{\nu \sigma^{2}}\right]+\sum_{i=1}^{n} \log \Phi\left[\frac{\lambda\left(y_{i}-\mathbf{x}_{i}^{\top} \boldsymbol{\beta}\right)}{\sigma}\right]
$$

onde $c(\nu)=\frac{2 \Gamma\left(\frac{\nu+1}{2}\right)}{\sqrt{\pi \nu} \Gamma\left(\frac{\nu}{2}\right)}$.

Como em Azzalini e Capitanio (2003), é possível encontrar a estimativa de máxima verossimilhança do vetor de parâmetros $\boldsymbol{\theta}$ maximizando diretamente a função log-verossimilhança. No entanto, esse procedimento é complicado, dado que existe uma integral envolvida $(\Phi()$.$) e uma solução alternativa através do algoritmo EM é proposta neste trabalho.$

Para implementar o algoritmo EM, primeiro note que, das propriedades conhecidas de 
uma variável aleatória t de Student, tem-se que

$$
\begin{aligned}
f(\mathbf{Y}) & =\prod_{i=2}^{m} 2 t_{\nu}\left(y_{i} \mid \mathbf{x}_{i}^{\top} \boldsymbol{\beta}, \sigma^{2}\right) \Phi\left(\lambda \frac{y_{i}-\mathbf{x}_{i}^{\top} \boldsymbol{\beta}}{\sigma^{2}}\right) \\
& =\prod_{i=2}^{n} 2 \int_{0}^{\infty} \int_{0}^{\infty} \phi\left(y_{i} \mid \mathbf{x}_{i}^{\top} \boldsymbol{\beta}, \sigma^{2} / u_{i}\right) G_{\frac{\nu}{2}, \frac{\nu}{2}}\left(u_{i}\right) \phi\left(t_{i} \mid \lambda y_{i}-\lambda \mathbf{x}_{i}^{\top} \boldsymbol{\beta}, \sigma^{2}\right) d t_{i} d u_{i},
\end{aligned}
$$

onde $G_{\frac{\nu}{2}, \frac{\nu}{2}}($.$) denota a fdp da distribuição Gama, com parâmetros \frac{\nu}{2}$ e $\frac{\nu}{2}, \nu>0$.

O modelo t-normal assimétrico tem a seguinte representação hierárquica

$$
\begin{aligned}
& Y_{i} \mid T_{i}=t_{i}, U_{i}=u_{i}, \stackrel{\text { ind }}{\sim} N\left(\mathbf{x}_{i}^{\top} \boldsymbol{\beta}+\frac{\sigma \lambda}{u_{i} \sqrt{1+\lambda^{2} / u_{i}}} t_{i}, \frac{\sigma^{2}}{u_{i}+\lambda^{2}}\right), \\
& U_{i} \stackrel{\text { ind }}{\sim} G_{\frac{\nu}{2}, \frac{\nu}{2}}\left(u_{i}\right) \\
& T_{i} \stackrel{\text { iid }}{\sim} N T(0,1), \quad i=1, \ldots, n,
\end{aligned}
$$

todos independentes, onde $N T(0,1)$ é a distribuição normal truncada padrão.

Sejam $\mathbf{Y}=\left(y_{1}, \ldots, y_{n}\right)^{\top}, \mathbf{u}=\left(u_{1}, \ldots, u_{n}\right)^{\top}$ e $\mathbf{t}=\left(t_{1}, \ldots, t_{n}\right)^{\top}$ e usando $\mathbf{u}$ e $\mathbf{t}$ como dados faltantes, segue que a função log-verossimilhança completa associada a $\mathbf{Y}_{c}=\left(\mathbf{Y}^{\top}, \mathbf{u}^{\top}, \mathbf{t}^{\top}\right)^{\top}$ é dada por

$$
\ell_{c}\left(\boldsymbol{\theta} \mid \mathbf{Y}_{c}\right) \propto-n \log \sigma^{2}-\frac{1}{2 \sigma^{2}} \sum_{i=1}^{n} u_{i}\left(y_{i}-\mathbf{x}_{i}^{\top} \boldsymbol{\beta}\right)^{2}-\frac{\nu}{2} \sum_{i=1}^{n} u_{i}-\frac{1}{2 \sigma^{2}} \sum_{i=1}^{n}\left(t_{i}-\lambda y_{i}+\lambda \mathbf{x}_{i}^{\top} \boldsymbol{\beta}\right)^{2} .
$$

Seja $\widehat{t_{i}}=\mathrm{E}\left[T_{i} \mid \boldsymbol{\theta}=\widehat{\boldsymbol{\theta}}, \mathbf{y}_{i}\right],{\widehat{t^{2}}}_{i}=\mathrm{E}\left[T_{i}^{2} \mid \boldsymbol{\theta}=\widehat{\boldsymbol{\theta}}, \mathbf{y}_{i}\right]$ e $\widehat{u}_{i}=\mathrm{E}\left[U_{i} \mid \boldsymbol{\theta}=\widehat{\boldsymbol{\theta}}, \mathbf{y}_{i}\right]$ obtém-se, usando os momentos da distribuição normal truncada (veja em Arellano-Valle et al., 2005), que

$$
\widehat{t_{i}}=\widehat{\lambda} \widehat{\eta}_{i}+\widehat{\sigma} W_{\Phi}\left(\frac{\widehat{\lambda} \widehat{\eta}_{i}}{\widehat{\sigma}}\right), \quad \widehat{t}^{2}{ }_{i}=\widehat{\lambda}^{2} \widehat{\eta}_{i}^{2}+\widehat{\sigma}^{2}+\widehat{\lambda} \widehat{\sigma} \widehat{\eta}_{i} W_{\Phi}\left(\frac{\widehat{\lambda} \widehat{\eta}_{i}}{\widehat{\sigma}}\right) \text { e } \widehat{u}_{i}=\frac{\nu+1}{\nu+e_{i}^{2}}
$$

onde $e_{i}=\frac{y_{i}-\mathbf{x}_{i}^{\top} \boldsymbol{\beta}}{\sigma}, W_{\Phi_{1}}(u)=\phi_{1}(u) / \Phi_{1}(u)$ e $\eta_{i}=y_{i}-\mathbf{x}_{i}^{\top} \boldsymbol{\beta}, i=1, \ldots, n$.

Denote por $\boldsymbol{\theta}^{(k)}$ o vetor $\left(\boldsymbol{\beta}^{(k)^{\top}}, \sigma^{2(k)}, \lambda^{(k)}\right)^{\top}$ a estimativa de $\boldsymbol{\theta}$ na a $k$-ésima iteração. Segue que a esperança com respeito a t, w, condicionada em $\mathbf{y}$, da função log-verossimilhança 
completa (Passo E), tem a forma

$$
\begin{aligned}
Q\left(\boldsymbol{\theta} \mid \widehat{\boldsymbol{\theta}}^{(k)}\right)= & \mathrm{E}\left[\ell_{c}\left(\boldsymbol{\theta} \mid \mathbf{y}_{c}\right) \mid \mathbf{y}, \widehat{\boldsymbol{\theta}}^{(k)}\right] \\
\propto & -n \log {\sigma^{2}}^{(k)}-\frac{1}{2 \sigma^{2(k)}} \sum_{i=1}^{n} \widehat{u}_{i}^{(k)}\left(y_{i}-\mathbf{x}_{i}^{\top} \boldsymbol{\beta}^{(k)}\right)^{2} \\
& -\frac{1}{2 \sigma^{2(k)}} \sum_{i=1}^{n}\left[\widehat{t}^{(k)}{ }^{(k)}-2 \lambda^{(k)} \widehat{t}_{i}^{(k)}\left(y_{i}-\mathbf{x}_{i}^{\top} \boldsymbol{\beta}^{(k)}\right)+\lambda^{(k)^{2}}\left(y_{i}-\mathbf{x}_{i}^{\top} \boldsymbol{\beta}^{(k)}\right)^{2}\right] .
\end{aligned}
$$

Portanto, tem-se o seguinte algoritmo EM:

Passo E: Dado $\boldsymbol{\theta}=\widehat{\boldsymbol{\theta}}^{(k)}$, calcule $\widehat{t}_{i}^{(k)},{\widehat{t^{2}}}^{(k)}$ e $\widehat{u}_{i}^{(k)}$, para $i=1, \ldots, n$, usando (3.10).

Passo M: Atualize $\widehat{\boldsymbol{\theta}}$ maximizando $Q\left(\boldsymbol{\theta} \mid \widehat{\boldsymbol{\theta}}^{(k)}\right)$ em relação a $\boldsymbol{\theta}$, que leva às seguintes expressões:

$$
\begin{aligned}
\widehat{\boldsymbol{\beta}}^{(k+1)} & \left.=\left[\mathbf{X}^{\top}\left(\mathbf{D}\left(\widehat{\mathbf{u}}^{(k)}\right)+\lambda^{(k)^{2}} \mathbb{I}_{n}\right) \mathbf{X}\right]^{-1} \mathbf{X}^{\top}\left[\mathbf{D}\left(\widehat{\mathbf{u}}^{(k)}\right) \mathbf{y}-\lambda^{(k)} \widehat{\mathbf{t}}^{(k)}-\lambda^{(k)} \mathbf{y}\right)\right], \\
{\widehat{\sigma^{2}}}^{(k+1)} & =\frac{1}{2 n}\left[Q_{\widehat{\mathbf{u}}^{(k)}}\left(\boldsymbol{\beta}^{(k)}\right)+\widehat{\mathbf{t}}^{(k)^{\top}} \mathbf{1}_{n}-2 \lambda^{(k)} \widehat{\mathbf{t}}^{(k)^{\top}}\left(\mathbf{y}-\mathbf{X} \boldsymbol{\beta}^{(k)}\right)+\lambda^{(k)^{2}} Q\left(\boldsymbol{\beta}^{(k)}\right)\right], \\
\widehat{\lambda}^{(k+1)} & =\frac{\widehat{\mathbf{t}}^{(k)^{\top}}\left(\mathbf{y}-\mathbf{X} \boldsymbol{\beta}^{(k)}\right)}{Q\left(\boldsymbol{\beta}^{(k)}\right)},
\end{aligned}
$$

onde $\mathbf{1}_{n}: n \times 1$ é um vetor de 1's, $\mathbb{I}_{n}$ é a matriz identidade de ordem $n, \mathbf{X}^{\top}=\left(\mathbf{x}_{1} \ldots, \mathbf{x}_{n}\right)$ é a matriz de planejamento, de dimensão $n \times p, Q\left(\boldsymbol{\beta}^{(k)}\right)=\left(\mathbf{y}-\mathbf{X} \boldsymbol{\beta}^{(k)}\right)^{\top}\left(\mathbf{y}-\mathbf{X} \boldsymbol{\beta}^{(k)}\right), Q_{\widehat{\mathbf{u}}^{(k)}}\left(\boldsymbol{\beta}^{(k)}\right)=$ $\left(\mathbf{y}-\mathbf{X} \boldsymbol{\beta}^{(k)}\right)^{\top} \mathbf{D}\left(\widehat{\mathbf{u}}^{(k)}\right)\left(\mathbf{y}-\mathbf{X} \boldsymbol{\beta}^{(k)}\right)$ e $\mathbf{D}\left(\widehat{\mathbf{u}}^{(k)}\right)=\operatorname{Diag}\left(\widehat{u}_{1}^{(k)}, \ldots, \widehat{u}_{n}^{(k)}\right)$.

O parâmetro $\nu$ é considerado conhecido em cada etapa do algoritmo EM. Em uma situação prática, $\nu$ pode ser estimado através de máxima verossimilhança perfilada, para um intervalo de valores de $\boldsymbol{\nu}=[1,2, \ldots, r e p]$ (por exemplo, rep $=30$ ).

\section{Ajuste dos dados gerados na Seção 2.8}

Na Seção 2.8 foi simulado um modelo normal assimétrico, com perturbações de alguns pontos no extremos da cauda pesada. Foi verificado que o modelo normal assimétrico não ajustou bem os dados, conclusão sugerida pela presença de pontos fora da banda de confiança do gráfico de envelope simulado. Ajustando o mesmo conjunto de dados utilizando a distribuição t-normal assimétrica, tem-se o gráfico de envelope simulado na Figura 3.2, demonstrando a robustez da distribuição t-normal assimétrica no ajuste de um conjunto de dados assimétricos e na presença de observações extremas.

Ferreira, C. S. 


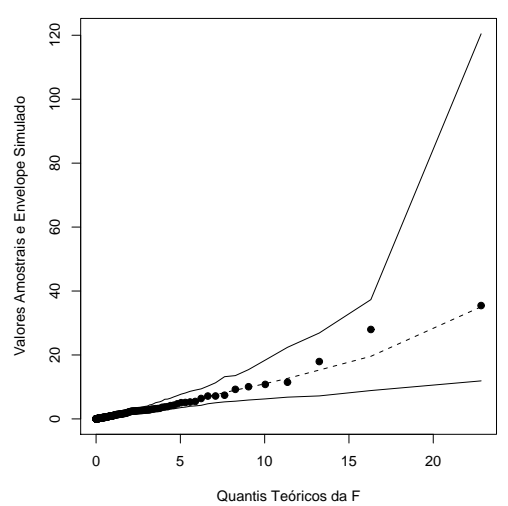

Figura 3.2: Envelope simulado para os dados simulados de uma população $N A(10,1,5)$, $n=200$, com pontos perturbados na cauda pesada da distribuição.

\subsection{Matriz de Informação Observada}

Considere $Y_{1}, \ldots, Y_{n}$, onde $Y_{i} \sim T N A\left(\mu_{i}, \sigma^{2}, \lambda\right), \mu_{i}=\mathbf{x}_{i}^{\top} \boldsymbol{\beta}, i=1, \ldots, n$, onde $\boldsymbol{\beta}$ é um vetor $p$-dimensional de coeficientes de regressão desconhecidos.

A função escore

$$
U(\boldsymbol{\theta})=\frac{\partial \ell(\boldsymbol{\theta})}{\partial \boldsymbol{\theta}}=\left(U(\boldsymbol{\beta})^{\top}, U\left(\sigma^{2}\right), U(\lambda)\right)^{\top}
$$

tem elementos $U(\boldsymbol{\theta})$ dados por

$$
\begin{aligned}
U(\boldsymbol{\beta}) & =\frac{\nu+1}{\nu \sigma^{2}} \boldsymbol{X}^{\top} \mathbf{D}(\mathbf{d})(\mathbf{y}-\boldsymbol{X} \boldsymbol{\beta})-\frac{\lambda}{\sigma} \boldsymbol{X}^{\top} \boldsymbol{a} \\
U\left(\sigma^{2}\right) & =-\frac{m}{2 \sigma^{2}}-\frac{\lambda}{2 \sigma^{3}}(\mathbf{y}-\boldsymbol{X} \boldsymbol{\beta})^{\top} \boldsymbol{a}+\frac{\nu+1}{2 \nu \sigma^{4}}(\mathbf{y}-\boldsymbol{X} \boldsymbol{\beta})^{\top} \mathbf{D}(\mathbf{d})(\mathbf{y}-\boldsymbol{X} \boldsymbol{\beta}) \mathrm{e} \\
U(\lambda) & =\frac{1}{\sigma}(\mathbf{y}-\boldsymbol{X} \boldsymbol{\beta})^{\top} \boldsymbol{a}
\end{aligned}
$$

onde $\mathbf{a}=\left(a_{1}, \cdots, a_{n}\right)^{\top}, a_{i}=W_{\phi}\left(B_{i}\right)$ e $D(\boldsymbol{d})=\operatorname{diag}\left(d_{1}, \ldots, d_{n}\right), \operatorname{com} d_{i}=\left(1+\frac{\left(y_{i}-\mathbf{x}_{i}^{\top} \boldsymbol{\beta}\right)^{2}}{\nu \sigma^{2}}\right)^{-1}$.

A matriz de informação de Fisher observada é dada por

$$
\mathbf{I}(\boldsymbol{\theta})=\left(\begin{array}{ccc}
I_{\boldsymbol{\beta} \boldsymbol{\beta}} & I_{\boldsymbol{\beta}_{\sigma^{2}}} & I_{\boldsymbol{\beta} \lambda} \\
& I_{\sigma^{2} \sigma^{2}} & I_{\sigma^{2} \lambda} \\
& & I_{\lambda \lambda}
\end{array}\right),
$$

Ferreira, C. S. 
com elementos

$$
\begin{aligned}
I_{\boldsymbol{\beta} \boldsymbol{\beta}}= & \mathbf{X}^{\top}\left[\frac{\nu+1}{\nu \sigma^{2}}\left(\frac{2}{\nu \sigma^{2}} D(\mathbf{d})^{2} D(\mathbf{y}-\mathbf{X} \boldsymbol{\beta})^{2}-D(\mathbf{d})\right)+\frac{\lambda^{2}}{\sigma^{2}} D(\mathbf{b})\right] \mathbf{X}, \\
I_{\boldsymbol{\beta} \sigma^{2}}= & \frac{(\nu+1)}{\nu \sigma^{4}}\left(-\mathbf{X}^{\top} D(\mathbf{d})(\mathbf{y}-\mathbf{X} \boldsymbol{\beta})+\frac{1}{\nu \sigma^{2}} \mathbf{X}^{\top} D(\mathbf{d})^{2} D(\mathbf{y}-\mathbf{X} \boldsymbol{\beta})^{2}(\mathbf{y}-\mathbf{X} \boldsymbol{\beta})\right) \\
& +\frac{\lambda}{2 \sigma^{3}}\left(\mathbf{X}^{\top} \mathbf{a}+\frac{\lambda}{\sigma} \mathbf{X}^{\top} D(\mathbf{b})(\mathbf{y}-\mathbf{X} \boldsymbol{\beta})\right), \\
I_{\boldsymbol{\beta} \lambda}= & -\frac{1}{\sigma}\left(\mathbf{X}^{\top} \mathbf{a}+\frac{\lambda}{\sigma} \mathbf{X}^{\top} D(\mathbf{b})(\mathbf{y}-\mathbf{X} \boldsymbol{\beta})\right), \\
I_{\sigma^{2} \sigma^{2}=}= & \frac{\nu+1}{2 \nu \sigma^{6}}\left(\frac{1}{\nu \sigma^{2}}(\mathbf{y}-\mathbf{X} \boldsymbol{\beta})^{\top} D(\mathbf{d})^{2} D(\mathbf{y}-\mathbf{X} \boldsymbol{\beta})^{2}(\mathbf{y}-\mathbf{X} \boldsymbol{\beta})-2(\mathbf{y}-\mathbf{X} \boldsymbol{\beta})^{\top} D(\mathbf{d})(\mathbf{y}-\mathbf{X} \boldsymbol{\beta})\right) \\
& +\frac{\lambda}{4 \sigma^{5}}\left(3(\mathbf{y}-\mathbf{X} \boldsymbol{\beta})^{\top} \mathbf{a}+\frac{\lambda}{\sigma}(\mathbf{y}-\mathbf{X} \boldsymbol{\beta})^{\top} D(\mathbf{b})(\mathbf{y}-\mathbf{X} \boldsymbol{\beta})\right)+\frac{n}{2 \sigma^{4}}, \\
I_{\sigma^{2} \lambda}= & -\frac{1}{2 \sigma^{3}}\left((\mathbf{y}-\mathbf{X} \boldsymbol{\beta})^{\top} \boldsymbol{a}+\frac{\lambda}{\sigma}(\mathbf{y}-\mathbf{X} \boldsymbol{\beta})^{\top} D(\mathbf{b})(\mathbf{y}-\mathbf{X} \boldsymbol{\beta})\right) \mathrm{e} \\
I_{\lambda \lambda}= & \frac{1}{\sigma^{2}}(\mathbf{y}-\mathbf{X} \boldsymbol{\beta})^{\top} D(\mathbf{b})(\mathbf{y}-\mathbf{X} \boldsymbol{\beta}),
\end{aligned}
$$

onde $\mathbf{b}^{\top}=\left(b_{1}, \cdots, b_{n}\right), b_{i}=W_{\phi}^{\prime}\left(B_{i}\right)=-W_{\phi}\left(B_{i}\right)\left(B_{i}+W_{\phi}\left(B_{i}\right)\right), \operatorname{com} B_{i}=\frac{\lambda\left(y_{i}-\mathbf{x}_{i}^{\top} \boldsymbol{\beta}\right)}{\sigma}, i=$ $1, \ldots, n$, e $W_{\phi}(u)=\phi_{1}(u) / \Phi_{1}(u), u \in \mathbb{R}$. A matriz de informação de Fisher esperada se encontra no Apêndice B.

\subsection{Diagnóstico no Modelo t-Normal Assimétrico}

Nesta seção serão calculadas a matriz hessiana $\left(\ddot{Q}_{\boldsymbol{\theta}}(\widehat{\boldsymbol{\theta}})\right)$ e as matrizes $\boldsymbol{\Delta}$ para análise de diagnósticos, sob o enfoque de Zhu e Lee (2001). As perturbações consideradas são ponderações de casos, perturbação no parâmetro de escala $\sigma^{2}$, perturbação no parâmetro de assimetria $\lambda$, perturbação em uma variável explicativa e perturbação na variável resposta. As matrizes $\boldsymbol{\Delta}$ são iguais as do modelo normal assimétrico, se $\widehat{u}=1$. Também é calculada a matriz $\ddot{Q}_{\boldsymbol{\theta}, \mathbf{y}}(\widehat{\boldsymbol{\theta}})$ de alavancagem.

\subsubsection{A matriz hessiana}

Para obter as medidas de diagnósticos baseadas na metodologia de Zhu e Lee (2001), é necessário calcular $\ddot{Q}_{\boldsymbol{\theta}}(\widehat{\boldsymbol{\theta}})=\left.\frac{\partial^{2} Q(\boldsymbol{\theta} \mid \widehat{\boldsymbol{\theta}})}{\partial \boldsymbol{\theta} \partial \boldsymbol{\theta}^{\top}}\right|_{\boldsymbol{\theta}=\widehat{\boldsymbol{\theta}}}$, onde $\boldsymbol{\theta}=\left(\boldsymbol{\beta}^{\top}, \sigma^{2}, \lambda\right)^{\top}$. Segue de (3.11) que 
$\ddot{Q}_{\boldsymbol{\theta}}(\widehat{\boldsymbol{\theta}})$ tem elementos dados por

$$
\begin{aligned}
\frac{\partial^{2} Q(\boldsymbol{\theta} \mid \widehat{\boldsymbol{\theta}})}{\partial \boldsymbol{\beta} \partial \boldsymbol{\beta}^{\top}} & =-\frac{1}{\sigma^{2}} \mathbf{X}^{\top}\left(\mathbf{D}(\widehat{\mathbf{u}})+\lambda^{2} \mathbb{I}_{n}\right) \mathbf{X}, \\
\frac{\partial^{2} Q(\boldsymbol{\theta} \mid \widehat{\boldsymbol{\theta}})}{\partial \boldsymbol{\beta} \partial \sigma^{2}} & =-\frac{1}{\sigma^{4}}\left[\mathbf{X}^{\top} \mathbf{D}(\widehat{\mathbf{u}})(\mathbf{y}-\mathbf{X} \boldsymbol{\beta})-\lambda \mathbf{X}^{\top}(\widehat{\mathbf{t}}-\lambda(\mathbf{y}-\mathbf{X} \boldsymbol{\beta}))\right], \\
\frac{\partial^{2} Q(\boldsymbol{\theta} \mid \widehat{\boldsymbol{\theta}})}{\partial \boldsymbol{\beta} \partial \lambda} & =\frac{1}{\sigma^{2}} \mathbf{X}^{\top}[2 \lambda(\mathbf{y}-\mathbf{X} \boldsymbol{\beta})-\widehat{\mathbf{t}}], \\
\frac{\partial^{2} Q(\boldsymbol{\theta} \mid \widehat{\boldsymbol{\theta}})}{\partial \sigma^{2} \partial \sigma^{2}} & =-\frac{1}{\sigma^{4}}\left[-n+\frac{1}{\sigma^{2}} Q_{\mathbf{u}}(\boldsymbol{\beta})+\frac{1}{\sigma^{2}}\left(\widehat{\mathbf{t}}^{\top} \mathbf{1}_{n}-2 \lambda(\mathbf{y}-\mathbf{X} \boldsymbol{\beta})^{\top} \widehat{\mathbf{t}}+\lambda^{2} Q(\boldsymbol{\beta})\right)\right], \\
\frac{\partial^{2} Q(\boldsymbol{\theta} \mid \widehat{\boldsymbol{\theta}})}{\partial^{2} \lambda} & =-\frac{1}{\sigma^{2}} Q(\boldsymbol{\beta}) \mathrm{e} \\
\frac{\partial^{2} Q(\boldsymbol{\theta} \mid \widehat{\boldsymbol{\theta}})}{\partial \sigma^{2} \partial \lambda} & =-\frac{1}{\sigma^{4}}\left[(\mathbf{y}-\mathbf{X} \boldsymbol{\beta})^{\top} \widehat{\mathbf{t}}-\lambda Q(\boldsymbol{\beta})\right],
\end{aligned}
$$

onde $\widehat{\mathbf{t}^{2}}=\left[\widehat{t}^{2}{ }_{1}, \ldots, \widehat{t}^{2}{ }_{n}\right]^{\top}$.

\subsubsection{Perturbação de ponderação de casos}

Seja $\boldsymbol{\omega}=\left(\omega_{1}, \ldots, \omega_{n}\right)^{\top}$ um vetor $n \times 1$ com $\boldsymbol{\omega}_{0}=(1, \ldots, 1)^{\top}$. Então, o valor esperado da função log-verossimilhança perturbada dos dados completos (função $Q$ perturbada) pode ser escrita como

$$
Q(\boldsymbol{\theta}, \boldsymbol{\omega} \mid \widehat{\boldsymbol{\theta}})=\sum_{i=1}^{n} \omega_{i} Q_{i}(\boldsymbol{\theta} \mid \widehat{\boldsymbol{\theta}})
$$

Nesse caso a matriz $\boldsymbol{\Delta}_{\boldsymbol{\omega}_{0}}=\left.\frac{\partial^{2} Q(\boldsymbol{\theta}, \boldsymbol{\omega} \mid \widehat{\boldsymbol{\theta}})}{\partial \boldsymbol{\theta} \partial \boldsymbol{\omega}^{\top}}\right|_{\boldsymbol{\omega}=\boldsymbol{\omega}_{0}}=\frac{\partial^{2} Q\left(\boldsymbol{\theta}, \boldsymbol{\omega}_{0} \mid \widehat{\boldsymbol{\theta}}\right)}{\partial \boldsymbol{\theta} \partial \boldsymbol{\omega}^{\top}}$ tem elementos dados por

$$
\begin{aligned}
\frac{\partial^{2} Q\left(\boldsymbol{\theta}, \boldsymbol{\omega}_{0} \mid \widehat{\boldsymbol{\theta}}\right)}{\partial \boldsymbol{\beta} \partial \boldsymbol{\omega}^{\top}}= & \frac{1}{\sigma^{2}} \mathbf{X}^{\top}\left[\mathbf{D}(\widehat{\mathbf{u}}) \mathbf{D}(\mathbf{y}-\mathbf{X} \boldsymbol{\beta})-\lambda \mathbf{D}(\widehat{\mathbf{t}})+\lambda^{2} \mathbf{D}(\mathbf{y}-\mathbf{X} \boldsymbol{\beta})\right] \\
\frac{\partial^{2} Q\left(\boldsymbol{\theta}, \boldsymbol{\omega}_{0} \mid \widehat{\boldsymbol{\theta}}\right)}{\partial \sigma^{2} \partial \boldsymbol{\omega}^{\top}}= & -\frac{1}{\sigma^{2}} \mathbf{1}_{n}^{\top}+\frac{1}{2 \sigma^{4}}\left[(\mathbf{y}-\mathbf{X} \boldsymbol{\beta})^{\top}\left(\mathbf{D}(\widehat{\mathbf{u}})+\lambda^{2} \mathbb{I}_{n}\right) \mathbf{D}(\mathbf{y}-\mathbf{X} \boldsymbol{\beta})\right. \\
& \left.+\widehat{\mathbf{t}}^{\top}-2 \lambda(\mathbf{y}-\mathbf{X} \boldsymbol{\beta})^{\top} \mathbf{D}(\widehat{\mathbf{t}})\right] \mathrm{e} \\
\frac{\partial^{2} Q\left(\boldsymbol{\theta}, \boldsymbol{\omega}_{0} \mid \widehat{\boldsymbol{\theta}}\right)}{\partial \lambda \partial \boldsymbol{\omega}^{\top}}= & \frac{1}{\sigma^{2}}(\mathbf{y}-\mathbf{X} \boldsymbol{\beta})^{\top}[\mathbf{D}(\widehat{\mathbf{t}})-\lambda \mathbf{D}(\mathbf{y}-\mathbf{X} \boldsymbol{\beta})]
\end{aligned}
$$

Ferreira, C. S. 


\subsubsection{Perturbação do parâmetro de escala}

Esse esquema de perturbação é introduzido considerando o modelo

$$
y_{i} \sim T N A_{\nu}\left(\mathbf{x}_{i}^{\top} \boldsymbol{\beta}, \omega_{i}^{-1} \sigma^{2}, \lambda\right), \omega_{i}>0, i=1, \ldots, n
$$

A função $Q$ perturbada é da forma

$$
\begin{aligned}
Q(\boldsymbol{\theta}, \mathbf{w} \mid \widehat{\boldsymbol{\theta}}) \propto & -\sum_{i=1}^{n} \log \frac{\sigma^{2}}{\omega_{i}}-\frac{1}{2 \sigma^{2}} \sum_{i=1}^{n} \omega_{i} \widehat{u}_{i}\left(y_{i}-\mathbf{x}_{i}^{\top} \boldsymbol{\beta}\right)^{2} \\
& -\frac{1}{2 \sigma^{2}} \sum_{i=1}^{n} \omega_{i}\left[\widehat{t}^{2}{ }_{i}-2 \lambda \widehat{t}_{i}\left(y_{i}-\mathbf{x}_{i}^{\top} \boldsymbol{\beta}\right)+\lambda^{2}\left(y_{i}-\mathbf{x}_{i}^{\top} \boldsymbol{\beta}\right)^{2}\right] .
\end{aligned}
$$

Sob esse esquema de perturbação o modelo não perturbado segue tomando $\boldsymbol{\omega}_{0}=(1, \ldots, 1)^{\top}$ e a matriz $\boldsymbol{\Delta}_{\boldsymbol{\omega}_{0}}$ é dada por

$$
\boldsymbol{\Delta} \boldsymbol{\omega}_{0}=\frac{1}{\sigma^{2}}\left[\begin{array}{l}
\mathbf{X}^{\top}\left(\mathbf{D}(\widehat{\mathbf{u}})+\lambda^{2} \mathbb{I}_{n}\right) \mathbf{D}(\mathbf{y}-\mathbf{X} \boldsymbol{\beta})-\lambda \mathbf{X}^{\top} \mathbf{D}(\widehat{\mathbf{t}}) \\
\frac{1}{2 \sigma^{2}}\left[(\mathbf{y}-\mathbf{X} \boldsymbol{\beta})^{\top}\left(\mathbf{D}(\widehat{\mathbf{u}})+\lambda^{2} \mathbb{I}_{n}\right) \mathbf{D}(\mathbf{y}-\mathbf{X} \boldsymbol{\beta})-2 \lambda(\mathbf{y}-\mathbf{X} \boldsymbol{\beta})^{\top} \mathbf{D}(\widehat{\mathbf{t}})+\widehat{\mathbf{t}}^{\top}\right. \\
(\mathbf{y}-\mathbf{X} \boldsymbol{\beta})^{\top}(\mathbf{D}(\widehat{\mathbf{t}})-\lambda \mathbf{D}(\mathbf{y}-\mathbf{X} \boldsymbol{\beta}))
\end{array}\right] .
$$

\subsubsection{Perturbação do parâmetro de assimetria}

Esse esquema de perturbação é introduzido considerando o modelo

$$
y_{i} \sim T N A_{\nu}\left(\mathbf{x}_{i}^{\top} \boldsymbol{\beta}, \sigma^{2}, \lambda \omega_{i}\right), \omega_{i} \in \mathbb{R}, i=1, \ldots, n .
$$

Sendo $\boldsymbol{\omega}=\left(w_{1}, \ldots, w_{n}\right)^{\top}$, a função $Q$ perturbada é da forma

$$
\begin{aligned}
Q(\boldsymbol{\theta}, \boldsymbol{\omega} \mid \widehat{\boldsymbol{\theta}}) \propto & -n \log \sigma^{2}-\frac{1}{2 \sigma^{2}} Q_{\mathbf{u}}(\boldsymbol{\beta}) \\
& -\frac{1}{2 \sigma^{2}} \sum_{i=1}^{n}\left[\widehat{t}^{2}{ }_{i}-2 \lambda \omega_{i} \widehat{t}_{i}\left(y_{i}-\mathbf{x}_{i}^{\top} \boldsymbol{\beta}\right)+\lambda^{2} \omega_{i}^{2}\left(y_{i}-\mathbf{x}_{i}^{\top} \boldsymbol{\beta}\right)^{2}\right] .
\end{aligned}
$$

Ferreira, C. S. 
Sob esse esquema de perturbação o modelo não perturbado segue considerando $\boldsymbol{\omega}_{0}=$ $(1, \ldots, 1)^{\top}$ e a matriz $\boldsymbol{\Delta}_{\boldsymbol{\omega}_{0}}$ é dada por

$$
\boldsymbol{\Delta}_{\boldsymbol{\omega}_{0}}=\frac{1}{\sigma^{2}}\left[\begin{array}{l}
\lambda \mathbf{X}^{\top}(2 \lambda \mathbf{D}(\mathbf{y}-\mathbf{X} \boldsymbol{\beta})-\mathbf{D}(\widehat{\mathbf{t}})) \\
\frac{\lambda}{\sigma^{2}}(\mathbf{y}-\mathbf{X} \boldsymbol{\beta})^{\top}[\lambda \mathbf{D}(\mathbf{y}-\mathbf{X} \boldsymbol{\beta})-\mathbf{D}(\widehat{\mathbf{t}})] \\
(\mathbf{y}-\mathbf{X} \boldsymbol{\beta})^{\top}(\mathbf{D}(\widehat{\mathbf{t}})-2 \lambda \mathbf{D}(\mathbf{y}-\mathbf{X} \boldsymbol{\beta}))
\end{array}\right] .
$$

\subsubsection{Perturbação na variável explicativa}

Nesse caso o interesse está em perturbar uma variável explicativa específica permitindo, por exemplo, detectar possíveis maus condicionamentos de alguma coluna da matriz de desenho (Belsley, 1991). Sob essa condição, tem-se a seguinte o esquema de perturbação aditiva em uma variável explicativa $\left(X_{v}\right)$

$$
x_{i v}(\boldsymbol{\omega})=x_{i v}+S_{v} \omega_{i}, \quad v \in\{1, \ldots, p\}, \omega_{i} \in \mathbb{R}, i=1, \ldots, n .
$$

onde $S_{v}$ é o desvio padrão da variável explicativa $X_{v}$. Nesse caso, $\boldsymbol{\omega}_{0}=\mathbf{0}$ e a função $Q$ perturbada é dada por

$$
\begin{aligned}
Q(\boldsymbol{\theta}, \boldsymbol{\omega} \mid \widehat{\boldsymbol{\theta}}) \propto & -n \log \sigma^{2}-\frac{1}{2 \sigma^{2}}\left(\mathbf{y}-\mathbf{X}_{\boldsymbol{\omega} \boldsymbol{\beta}}\right)^{\top} \mathbf{D}(\widehat{\mathbf{u}})\left(\mathbf{y}-\mathbf{X}_{\boldsymbol{\omega}} \boldsymbol{\beta}\right) \\
& -\frac{1}{2 \sigma^{2}} \widehat{\mathbf{t}}^{\top} \mathbf{1}_{n}+\frac{\lambda}{\sigma^{2}}\left(\mathbf{y}-\mathbf{X}_{\boldsymbol{\omega}} \boldsymbol{\beta}\right)^{\top} \widehat{\mathbf{t}}-\frac{\lambda^{2}}{2 \sigma^{2}}\left(\mathbf{y}-\mathbf{X}_{\boldsymbol{\omega}} \boldsymbol{\beta}\right)^{\top}\left(\mathbf{y}-\mathbf{X}_{\boldsymbol{\omega}} \boldsymbol{\beta}\right) .
\end{aligned}
$$

onde $\mathbf{X}_{\boldsymbol{\omega}}=\mathbf{X}+S_{v} \mathbf{A}_{v}$, onde $\mathbf{A}_{v}$ é uma matriz de zeros com a $v$-ésima coluna de 1's. Segue que a matriz $\boldsymbol{\Delta}_{\boldsymbol{\omega}_{0}}$ tem a forma

$$
\boldsymbol{\Delta}_{\boldsymbol{\omega}_{0}}=\frac{S_{v}}{\sigma^{2}}\left[\begin{array}{l}
\mathbf{0}_{v}^{1}(\mathbf{y}-\mathbf{X} \boldsymbol{\beta})^{\top}\left[\mathbf{D}(\widehat{\mathbf{u}})+\lambda^{2} \mathbb{I}_{n}\right]-\beta_{v} \mathbf{X}^{\top}\left[\mathbf{D}(\widehat{\mathbf{u}})+\lambda^{2} \mathbb{I}_{n}\right]-\lambda \mathbf{0}_{v}^{1} \widehat{\mathbf{t}}^{\top} \\
\frac{\boldsymbol{\beta}_{v}}{\sigma^{2}}\left(-(\mathbf{y}-\mathbf{X} \boldsymbol{\beta})^{\top} \mathbf{D}(\widehat{\mathbf{u}})+\lambda \widehat{\mathbf{t}}^{\top}-\lambda^{2}(\mathbf{y}-\mathbf{X} \boldsymbol{\beta})^{\top}\right) \\
\beta_{v}\left(-\widehat{\mathbf{t}}^{\top}+2 \lambda(\mathbf{y}-\mathbf{X} \boldsymbol{\beta})^{\top}\right)
\end{array}\right],
$$

onde $\mathbf{0}_{v}^{1}$ denota um vetor $p \times 1$ de zeros com 1 na $v$-ésima posição.

\subsubsection{Perturbação na variável resposta}

Uma perturbação aditiva na variável resposta $\mathbf{y}=\left(y_{1}, \ldots, y_{n}\right)^{\top}$ é introduzida substituindo $y_{i}$ por $y_{i \boldsymbol{\omega}}=y_{i}+\omega_{i} S_{y}, \quad i=1, \ldots, n$, onde $S_{y}$ é o desvio padrão de $\mathbf{y}$. Neste caso 
$\boldsymbol{\omega}_{0}=\mathbf{0} \mathrm{e}$

$$
\begin{aligned}
Q(\boldsymbol{\theta}, \boldsymbol{\omega} \mid \widehat{\boldsymbol{\theta}}) \propto & -n \log \sigma^{2}-\frac{1}{2 \sigma^{2}}(\mathbf{y} \boldsymbol{\omega}-\mathbf{X} \boldsymbol{\beta})^{\top} \mathbf{D}(\widehat{\mathbf{u}})(\mathbf{y} \boldsymbol{\omega}-\mathbf{X} \boldsymbol{\beta}) \\
& -\frac{1}{2 \sigma^{2}} \widehat{\mathbf{t}}^{\top} \mathbf{1}_{n}+\frac{\lambda}{\sigma^{2}}(\mathbf{y} \boldsymbol{\omega}-\mathbf{X} \boldsymbol{\beta})^{\top} \widehat{\mathbf{t}}-\frac{\lambda^{2}}{2 \sigma^{2}}(\mathbf{y} \boldsymbol{\omega}-\mathbf{X} \boldsymbol{\beta})^{\top}(\mathbf{y} \boldsymbol{\omega}-\mathbf{X} \boldsymbol{\beta}) .
\end{aligned}
$$

Segue que a matriz $\boldsymbol{\Delta} \boldsymbol{\omega}_{0}$ tem a forma

$$
\boldsymbol{\Delta}_{\boldsymbol{\omega}_{0}}=\frac{S_{y}}{\sigma^{2}}\left[\begin{array}{l}
\mathbf{X}^{\top}\left(\mathbf{D}(\widehat{\mathbf{u}})+\lambda^{2} \mathbb{I}_{n}\right) \\
\frac{1}{\sigma^{2}}\left((\mathbf{y}-\mathbf{X} \boldsymbol{\beta})^{\top} \mathbf{D}(\widehat{\mathbf{u}})+\lambda^{2}(\mathbf{y}-\mathbf{X} \boldsymbol{\beta})^{\top}-\lambda \widehat{\mathbf{t}}^{\top}\right) \\
\widehat{\mathbf{t}}^{\top}-2 \lambda(\mathbf{y}-\mathbf{X} \boldsymbol{\beta})^{\top}
\end{array}\right] .
$$

\subsection{Estudo de Dados Artificiais}

Para ilustrar a utilidade da metodologia proposta, nesta seção são apresentados resultados obtidos através de situações hipotéticas.

Um conjunto de dados foi simulado, com a presença de uma variável regressora, da forma $y=\alpha+\beta x+\sigma \varepsilon$, onde $\varepsilon \sim T N A_{\nu}(\lambda)$. A variável $x$ foi gerada da distribuição $U(0,1)$, com $n=$ 100. A Tabela 3.1 apresenta os valores simulados e estimados dos parâmetros, sob os modelos normal, normal assimétrico e t-normal assimétrico. Os erros padrão são estimados através da matriz de informação observada (Apêndice B). O critério de informação de Schwarz (ou equivalentemente a log-verossimilhança) foi usado para escolher entre alguns valores de $\nu$ como recomendado por Fernández e Steel (1999). Para valores de $\nu=0,1,2, \ldots, 100$,

calculou-se $\widehat{\boldsymbol{\theta}}_{\nu}$, ocorrendo o máximo de $l\left(\widehat{\boldsymbol{\theta}}_{\nu}\right)$, em $\nu=5$, seu valor simulado. Como $E[\varepsilon] \neq 0$, o resíduo para uma observação $y_{i}$ é dado por $e_{i}=y_{i}-\mathbf{x}_{i}^{\top} \widehat{\boldsymbol{\beta}}-\widehat{\sigma} \widehat{\varepsilon_{i}}$, onde $\widehat{\epsilon_{i}}$ é calculado na equação (3.4), para $k=1$, ajustado em $\hat{\lambda}$.

Analisando a Tabela 3.1, as estimativas de $\alpha$ e $\beta$ nos três modelos são parecidas. Por outro lado, as estimativas do parâmetros de escala $\sigma^{2}$ são diferentes, sendo menor no modelo normal e t-normal assimétrico. Realizando um teste de hipóteses assintótico (teste de razão de verossimilhança) para testar $H_{0}: \lambda=0 \times H_{1}: \lambda \neq 0$ (comparando modelo normal simétrico e assimétrico), tem-se que $R V=2\left(\ell(\widehat{\boldsymbol{\theta}})-\ell\left(\widehat{\boldsymbol{\theta}}_{0}\right)\right)=40.82$, com um p-valor $\ll$ 0.01, levando à rejeição da hipótese nula. Ou seja, um modelo normal assimétrico é mais apropriado que o modelo de regressão múltipla usual, assumindo erros normais. Pelos gráficos

Ferreira, C. S. 
Tabela 3.1: Modelos de regressão normal simétrico, normal assimétrico (NA) e t-normal assimétrico (TNA) com dados simulados: Estimativas dos parâmetros dos modelos, com seus respectivos erros padrão assintóticos entre parênteses.

\begin{tabular}{l|cccccc}
\hline \hline Modelos & $\alpha$ & $\beta$ & $\sigma^{2}$ & $\lambda$ & $\nu$ & $l(\boldsymbol{\theta})$ \\
\hline Valores verdadeiros & 10 & 2 & 1 & 5 & 5 & - \\
Normal & $10.69(0.16)$ & $2.32(0.27)$ & $0.60(0.08)$ & - & - & -116.03 \\
NA & $9.92(0.10)$ & $2.12(0.16)$ & $1.37(0.21)$ & $9.67(3.27)$ & - & -95.62 \\
TNA & $9.98(0.09)$ & $2.09(0.15)$ & $0.80(0.17)$ & $6.53(2.27)$ & 5 & -92.98 \\
\hline \hline
\end{tabular}

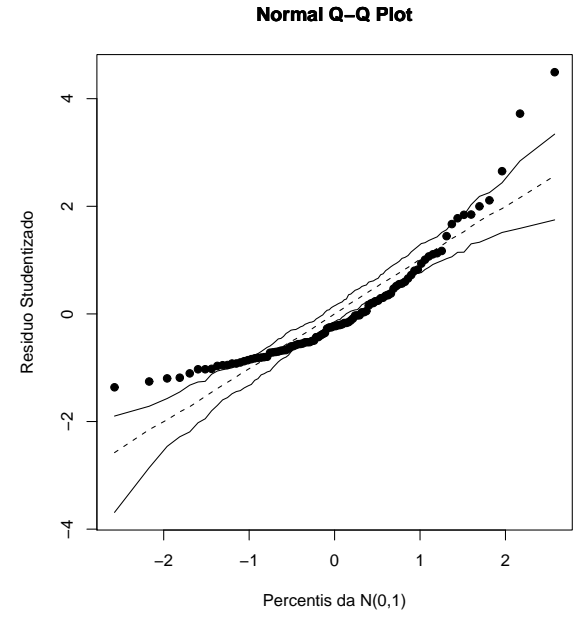

(a)

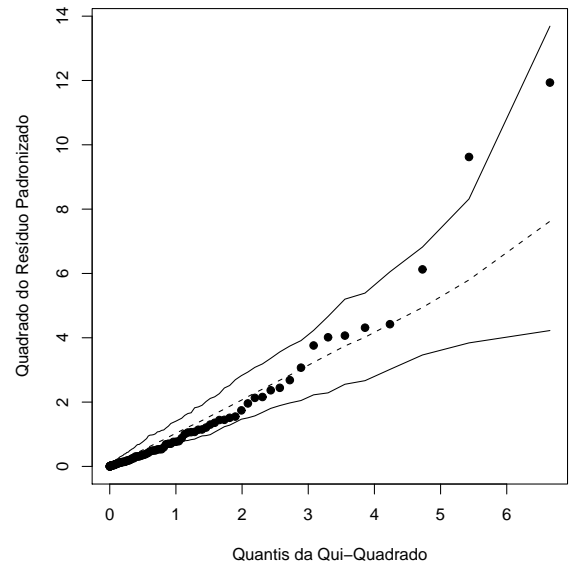

(b)

Figura 3.3: Gráficos de envelope simulado para o conjunto de dados simulados. (a) Modelo normal simétrico e (b) modelo normal assimétrico.

de envelopes (Figura 3.3), nota-se que esses dois modelos não conseguem incorporar todas as observações.

Por outro lado, comparando os modelos normal e t-normal assimétricos, o teste RV aponta uma diferença significativa $(R V=5.28$, p-valor $=0.02)$. O gráfico de envelope simulado (utilizando a representação dada na equação 3.5), não acusou pontos além da banda de confiança (Fig. 3.4b). Portanto, o modelo t-normal assimétrico é mais indicado ao conjunto de dados.

As análises de influência local e global detectaram três pontos influentes, um abaixo da reta estimada e dois acima. O ponto 57 (abaixo da reta) é o único influente em $D_{i}^{c}$, ponderação de casos e perturbação na escala. Os pontos 33 e 81 (acima da reta) são os 


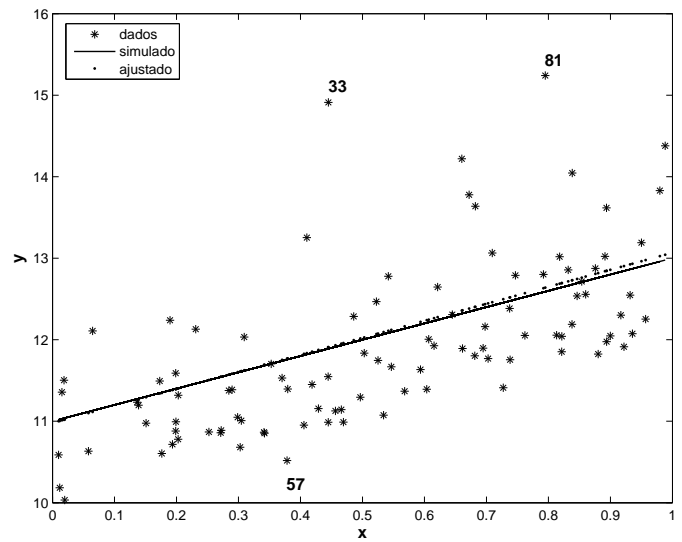

(a)

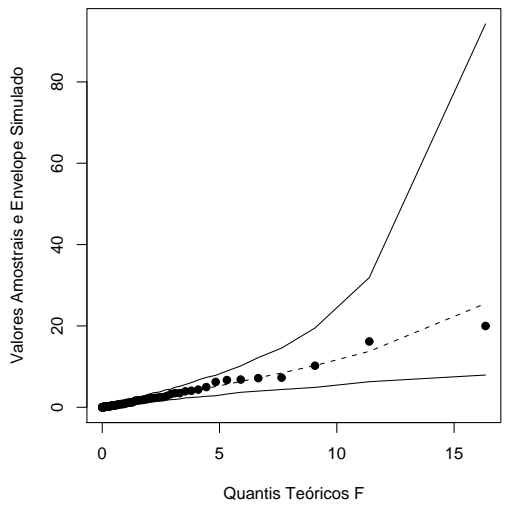

(b)

Figura 3.4: Modelo de regressão t-normal assimétrico com dados simulados. (a) Gráfico de dispersão, juntamente com as retas do modelo t-normal assimétrico simulado e ajustado e pontos influentes e (b) gráfico de envelope simulado.

pontos candidatos a influentes nas análises de perturbação na variável resposta, na variável explicativa e no parâmetro de assimetria $\lambda$. Os três pontos são influentes em $L D_{i}^{c}$. Ainda, os pontos 33 e 57 são candidatos a ponto de alavanca (além do 71).

Assim, pode-se pensar que a estrutura dos dados de uma distribuição $y \sim T N A_{\nu}(\alpha+$ $\left.\beta x, \sigma^{2}, \lambda\right)$ possui a característica de ter pontos influentes sob análise de influência global e local, porém não são pontos de alavanca (remotos no subespaço gerado pelas colunas da matriz X). Fazendo um exercício de excluir os pontos e calcular a mudança percentual $100\left[\widehat{\theta}_{i}(-k)-\widehat{\theta}_{i}\right] / \widehat{\theta}_{i}, i=1, \ldots, n$, nas estimativas dos parâmetros, percebe-se que as maiores mudanças são nos parâmetros $\lambda$ e $\sigma^{2}$ (Tabela 3.2).

Tabela 3.2: Dados simulados. Mudança nas estimativas dos parâmetros (em \%), excluindo os pontos (57), (33 e 81) e (33, 57 e 81).

\begin{tabular}{l|cccc}
\hline \hline Pontos excluídos & $\alpha$ & $\beta$ & $\sigma^{2}$ & $\lambda$ \\
\hline$(57)$ & 0.3 & -3.0 & 1.95 & 94.8 \\
$(33$ e 81$)$ & 0.19 & -0.42 & -14.18 & -11.99 \\
$(33,57$ e 81$)$ & 0.4 & -3.13 & -11.43 & 67.96 \\
\hline \hline
\end{tabular}

Os gráficos de envelope simulado para as três alterações acima apontaram pontos extremos somente quando retirou-se os pontos 33 e 81.

Ferreira, C. S. 


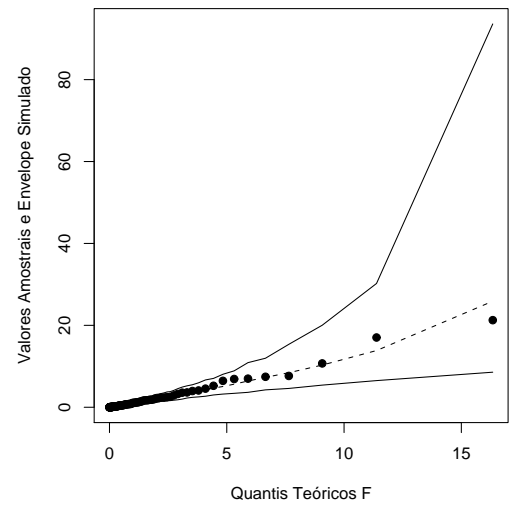

(a)

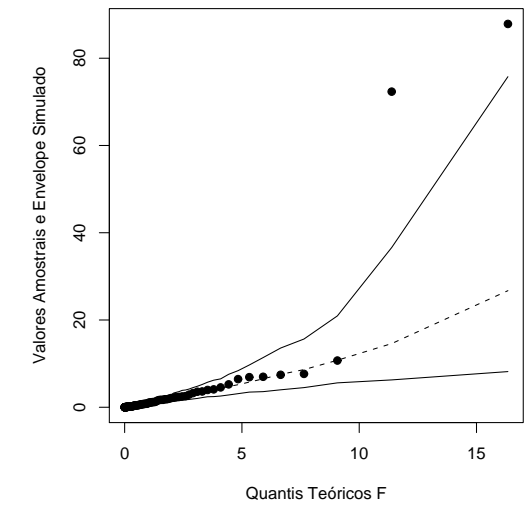

(b) (c)

Figura 3.5: Gráficos de envelopes: (a) Perturbação $y(57)=10$ e (b) perturbação $y(33)=$ $y(81)=19$.

Para analisar o comportamento das observações sob as medidas de influência abordadas neste trabalho, perturbou-se o ponto influente abaixo da reta, na forma $y(57)=10$. Depois perturbou-se os pontos influentes acima da reta estimada, na forma $y(33)=y(81)=19$.

Percebe-se que os coeficientes da regressão sofrem pouca mudança com as pertubações. Os parâmetros de escala $\left(\sigma^{2}\right)$ e assimetria $(\lambda)$ diminuem quando perturba-se o ponto abaixo da reta e aumentam quando perturba-se os dois pontos acima da reta (em relação ao estimado sem perturbação) (Tabela 3.3).

Tabela 3.3: Modelo de regressão TNA simulado: Estimativas do modelo, com e sem perturbações, com seus respectivos erros padrão assintóticos entre parênteses.

\begin{tabular}{l|ccccc}
\hline \hline & $\alpha$ & $\beta$ & $\sigma^{2}$ & $\lambda$ & $\nu$ \\
\hline Valores verdadeiros & 10 & 2 & 1 & 5 & 5 \\
Perturbação: $\mathrm{y}(57)=10$ & $10.00(0.13)$ & $2.13(0.19)$ & $0.74(0.17)$ & $3.04(0.89)$ & 5 \\
Perturbação: $\mathrm{y}(33)=\mathrm{y}(81)=19$ & $9.97(0.12)$ & $2.08(0.19)$ & $0.83(0.17)$ & $6.69(0.86)$ & 5 \\
\hline \hline
\end{tabular}

O gráfico de envelope para os dados perturbados indicou pontos além da banda de confiança somente na perturbação dos dois pontos acima da reta (Fig 3.5). A Tabela 3.4 apresenta os pontos influentes do exemplo com e sem perturbações.

Ferreira, C. S. 


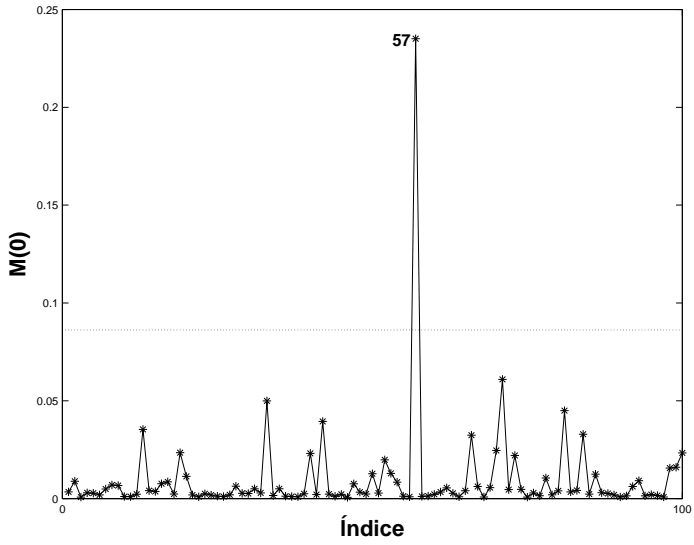

(a)

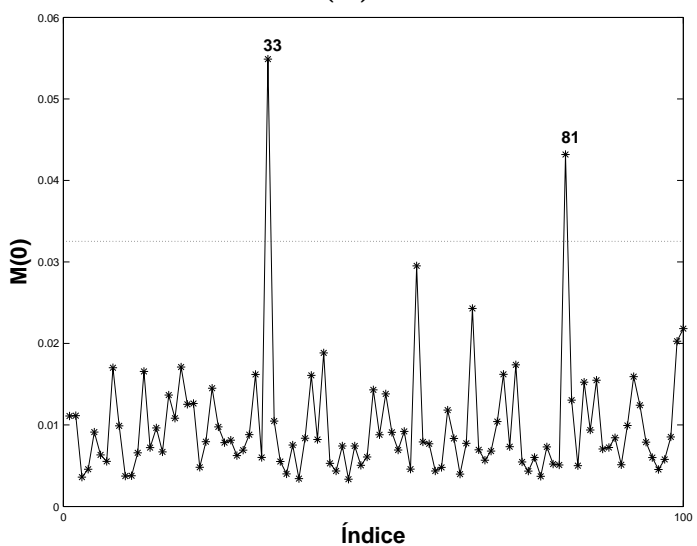

(c)

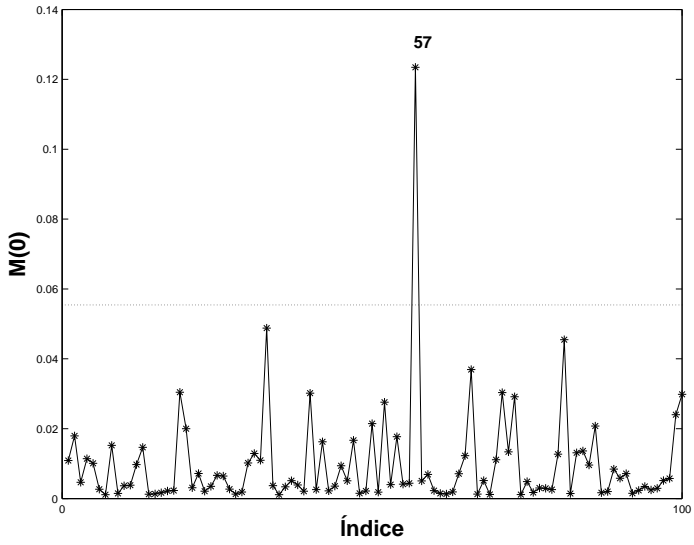

(b)

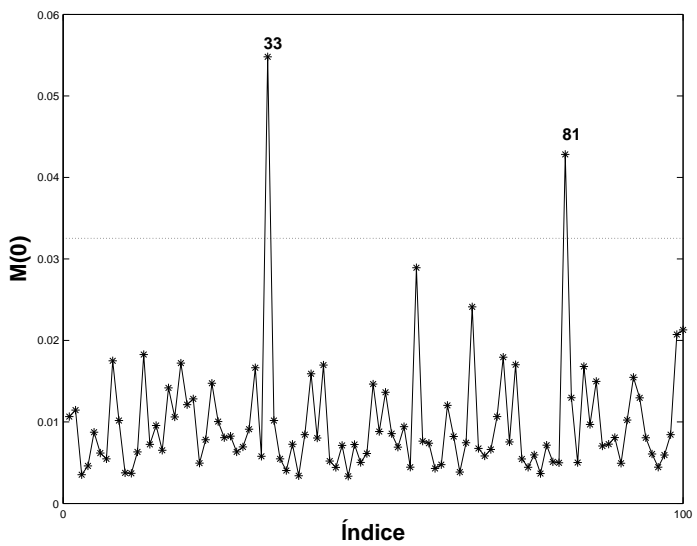

(d)

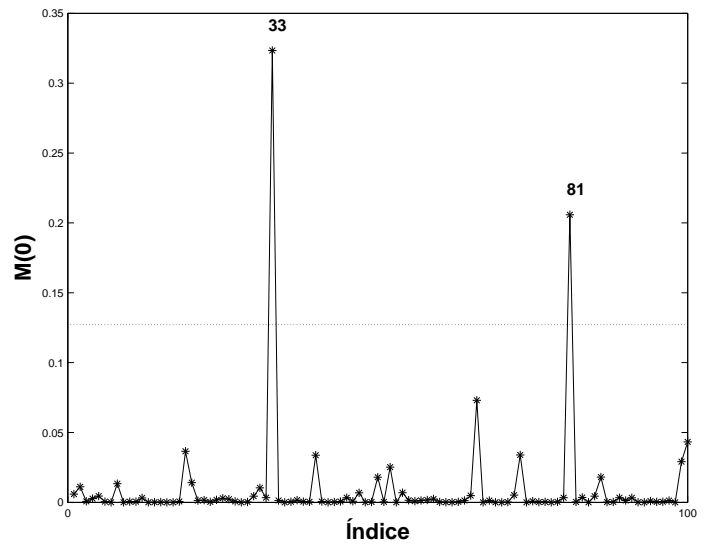

(e)

(f)

Figura 3.6: Gráficos de diagnóstico para o modelo de regressão TNA simulado, sem perturbações. Linhas horizontais são os pontos de corte $\left(c^{*}=3\right)$ : (a) Perturbação de ponderação de casos, (b) perturbação no parâmetro de escala, (c) perturbação na variável resposta, (d) perturbação na variável explicativa e (e) pertubação no parâmetro de assimetria. 


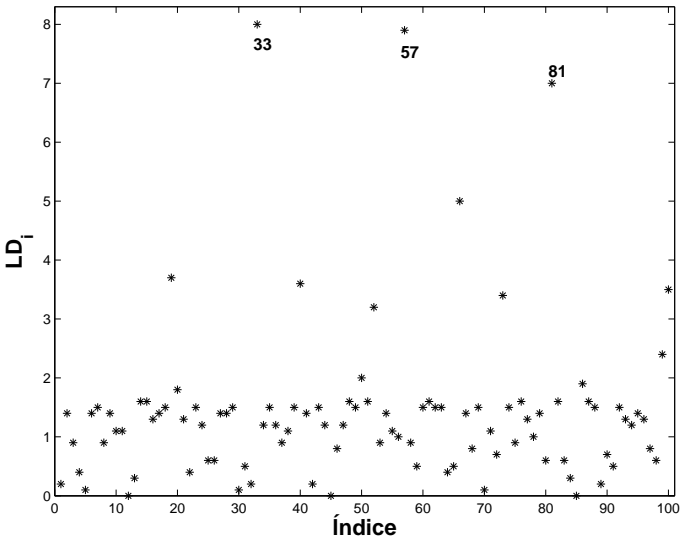

(a)

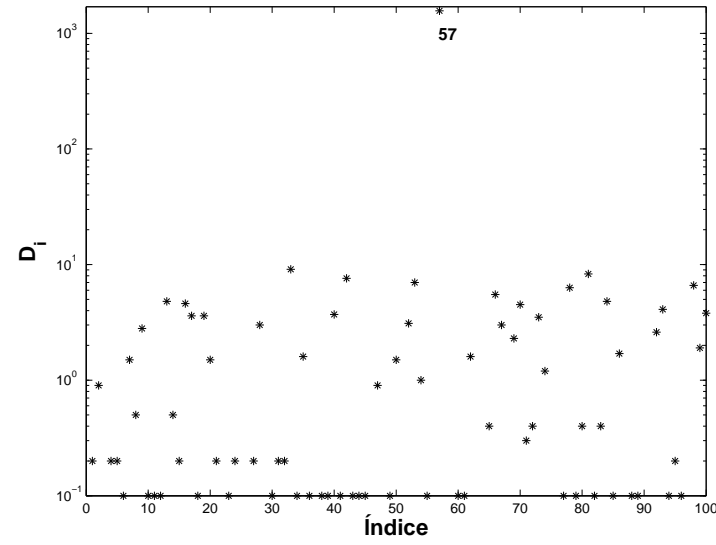

(b)

Figura 3.7: Influência global para o modelo de regressão TNA simulado, sem perturbações: (a) $L D_{i}^{c}$ e (b) $D_{i}^{c}$.

Tabela 3.4: Pontos influentes sob influência global e local, para o modelo de regressão TNA simulado, com e sem perturbações

\begin{tabular}{l|ccc}
\hline \hline & \multicolumn{3}{|c}{ Perturbações } \\
\hline Medidas de influência & Sem perturbar & $\mathrm{y}(57)=10$ & $\mathrm{y}(33)=\mathrm{y}(81)=19$ \\
\hline$D_{i}^{c}$ & 57 & 57 & 57 \\
$L D_{i}^{c}$ & $33,57,81$ & $33,57,81$ & $33,57,81$ \\
Alavanca & $33,57,71$ & $33,57,81$ & 33,81 \\
Ponderação de Casos & 57 & 57 & $33,57,81$ \\
Perturbação na escala & 57 & 57 & $33,57,81$ \\
Perturbação na assimetria & 33,81 & 33,81 & 33,81 \\
Perturbação na variável explicativa & 33,81 & 33,57 & 33,81 \\
Perturbação na variável resposta & 33,81 & $33,57,81$ & 33,81 \\
\hline \hline
\end{tabular}

Ferreira, C. S. 
Da Tabela 3.4, percebe-se alguns padrões. A medida de influência global $D_{i}^{c}$ detectou nas três análises apenas o ponto 57 , enquanto $L D_{i}^{c}$ detectou os três pontos. Ainda, a perturbação em $\lambda$ detectou apenas os pontos 33 e 81 nas três análises.

Por outro lado, ponderação de casos e perturbação na escala, que detectava apenas o ponto 57 no modelo simulado, continuou detectando apenas este ponto na perturbação do mesmo, enquanto ao perturbar os dois pontos acima da reta estimada, os três pontos foram detectados como influentes.

As perturbações nas variáveis explanatória e resposta detectam em geral como influentes pontos acima da reta estimada, porém pontos abaixo da reta podem ser detectados, caso a distância do ponto à massa seja expressiva. A medida de alavanca detectou os três pontos perturbados, abaixo e acima da reta.

As análises de influência levaram em conta o sinal positivo da assimetria. Na presença de assimetria negativa, a interpretação dos pontos acima e abaixo da reta estimada deve ser invertida. Os gráficos de influência dos modelos perturbados se encontram no Apêndice B.

\subsection{Aplicação}

Nesta seção o modelo de regressão t-normal assimétrico será aplicado a um subconjunto de variáveis do conjunto de dados do "Australian Institute of Sports"(AIS) (podendo ser baixado no endereço http://azzalini.stat.unipd.it/index-en.html). Um subconjunto desses dados foi previamente analisado em Azzalini e Capitanio (2003).

Tabela 3.5: Conjunto de dados AIS: Estatísticas descritivas para as variáveis "soma de manchas na pele"(ssf) e "porcentagem de gordura corporal" (bfat) dos indivíduos (EP é o erro padrão).

\begin{tabular}{l|ccccccc}
\hline \hline & Mínimo & Média & Máximo & Percentil 25 & Mediana & Percentil 75 & EP \\
\hline bfat & 5.63 & 13.51 & 35.32 & 8.54 & 11.65 & 18.08 & 6.19 \\
ssf & 28.00 & 69.02 & 200.80 & 43.80 & 58.60 & 90.40 & 32.57 \\
\hline \hline
\end{tabular}

Ferreira, C. S. 
Define-se o então modelo t-normal assimétrico

$$
\begin{aligned}
s s f_{i} & =\alpha+\beta \times b f a t_{i}+\sigma \varepsilon_{i} \\
\varepsilon_{i} & \stackrel{i i d}{\sim} T N A_{\nu}(\lambda), \quad i=1, \ldots, 202 .
\end{aligned}
$$

Arellano-Valle et al. (2005) ajustam uma medida de erro no modelo normal assimétrico para esse dado e notam uma forte relação entre variáveis e assimetria à direita. Os parâmetros estimados são dados na Tabela 3.6. O erro padrão foi calculado usando a matriz de informação esperada dada no Apêndice B. O critério de informação de Schwarz (ou equivalentemente a log-verossimilhança) foi usado para escolher entre alguns valores de $\nu$ como recomendado por Fernández e Steel (1999).

A estimação do parâmetro $\nu$ ocorreu da seguinte forma: Para cada valor de $\nu=$ $0,1,2, \ldots, 100$, estima-se $\widehat{\boldsymbol{\theta}}_{\nu}$ e $\widehat{\nu}$ é o valor de $\nu$ que maximiza $l\left(\widehat{\boldsymbol{\theta}}_{\nu}\right)$. Como $E[\varepsilon] \neq 0$, o resíduo para uma observação $y_{i}$ é dado por $e_{i}=y_{i}-\mathbf{x}_{i}^{\top} \widehat{\boldsymbol{\beta}}-\widehat{\sigma} \widehat{\varepsilon}_{i}$, onde $\widehat{\epsilon}_{i}$ é calculado na equação (3.4), para $k=1$, ajustado em $\widehat{\lambda}$. A Tabela 3.6 apresenta as estimativas do modelo t-normal assimétrico, juntamente com as estimativas do conjunto de dados sob os modelos normal simétrico e assimétrico.

Tabela 3.6: EMVs do modelo normal simétrico, modelo normal assimétrico (NA) e modelo t-normal assimétrico (TNA) para o conjunto de dados AIS (erro padrão estimado assintótico entre parênteses)

\begin{tabular}{lcccccc}
\hline \hline & $\widehat{\alpha}$ & $\widehat{\beta}$ & $\widehat{\sigma^{2}}$ & $\widehat{\lambda}$ & $\nu$ & $\ell(\widehat{\boldsymbol{\theta}})$ \\
\hline Normal & $0.59(1.48)$ & $5.07(0.10)$ & $76.61(58.14)$ & - & - & -1063.40 \\
NA & $-5.08(1.57)$ & $4.88(0.14)$ & $143.97(32.76)$ & $1.64(0.61)$ & - & -723.27 \\
TNA & $-2.06(1.87)$ & $4.78(0.14)$ & $91.18(27.09)$ & $1.08(0.58)$ & 8 & -721.97 \\
\hline \hline
\end{tabular}

Analisando a Tabela 3.6, as estimativas de $\beta$ nos três modelos são parecidas. Por outro lado, as estimativas do parâmetros de escala $\sigma^{2}$ são diferentes, sendo menor no modelo normal e t-normal assimétrico. Realizando um teste de hipóteses assintótico (teste de razão de verossimilhança) para testar $H_{0}: \lambda=0 \times H_{1}: \lambda \neq 0$ (comparando modelo normal simétrico e assimétrico), tem-se que $R V=2\left(\ell(\widehat{\boldsymbol{\theta}})-\ell\left(\widehat{\boldsymbol{\theta}}_{0}\right)\right)=680.26$, com um p-valor igual a 0 , levando à rejeição da hipótese nula. Ou seja, um modelo normal assimétrico é mais apropriado que o modelo de regressão múltipla usual, assumindo erros normais. Pelos gráficos 


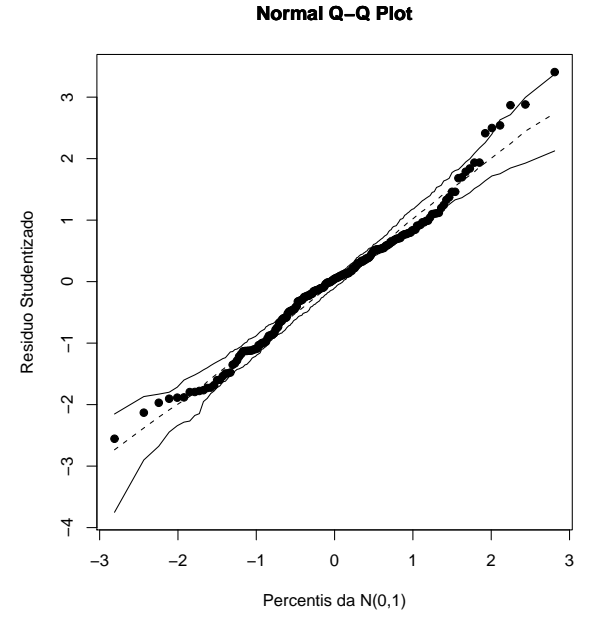

(a)

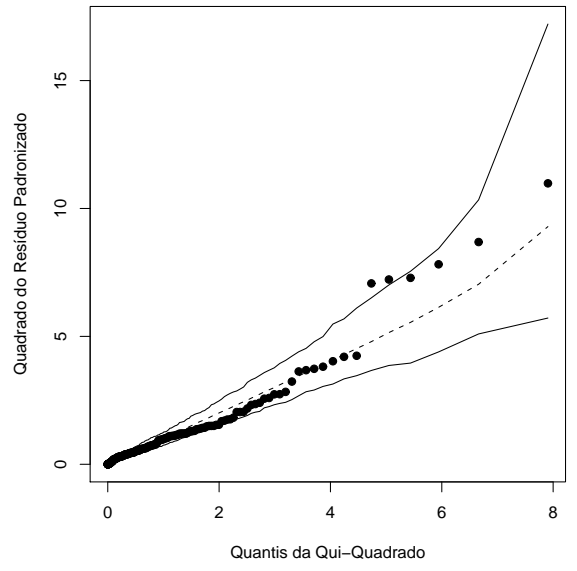

(b)

Figura 3.8: Gráficos de envelope simulado para o conjunto de dados AIS: (a) modelo normal simétrico e (b) modelo normal assimétrico.

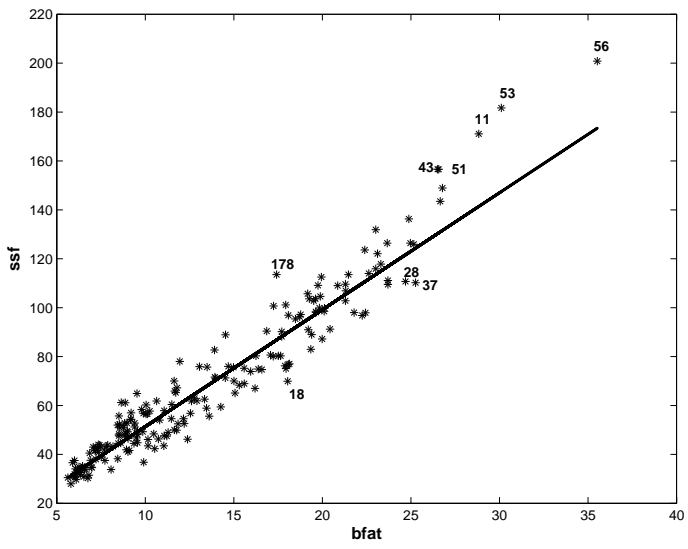

(a)

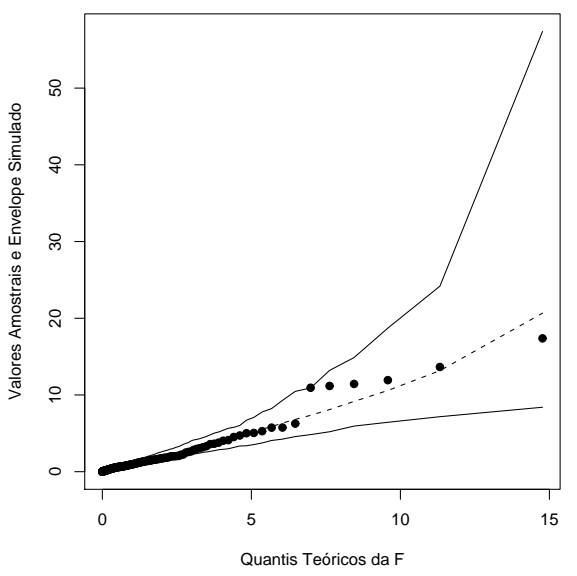

(b)

Figura 3.9: Ajuste de modelo de regressão t-normal assimétrico para os dados AIS. (a) Gráfico de dispersão, juntamente com a reta ajustada e pontos influentes e (b) gráfico de envelope simulado.

de envelopes (Figura 3.8), nota-se que esses dois modelos não conseguem incorporar todas as observações.

Por outro lado, comparando os modelos normal e t-normal assimétricos, o teste RV aponta uma diferença não significativa $(R V=2.6$, p-valor $=0.10)$. O gráfico de envelope simulado (utilizando a representação dada na equação 3.5), não acusou pontos além da banda 


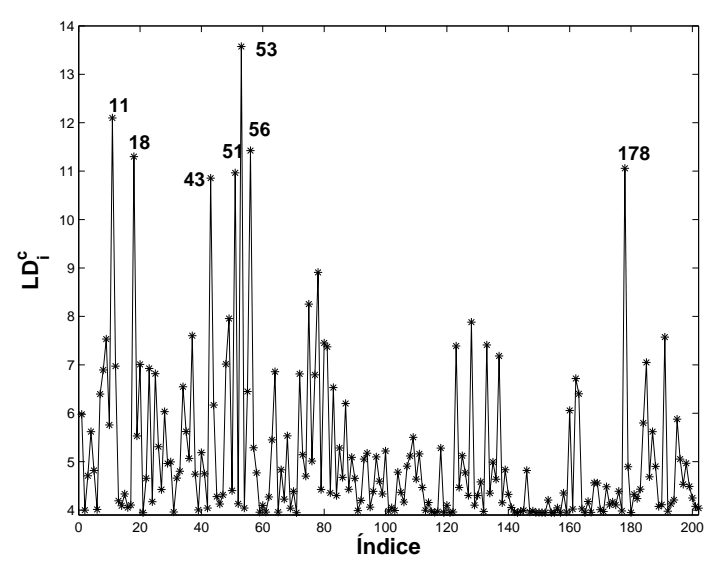

(a)

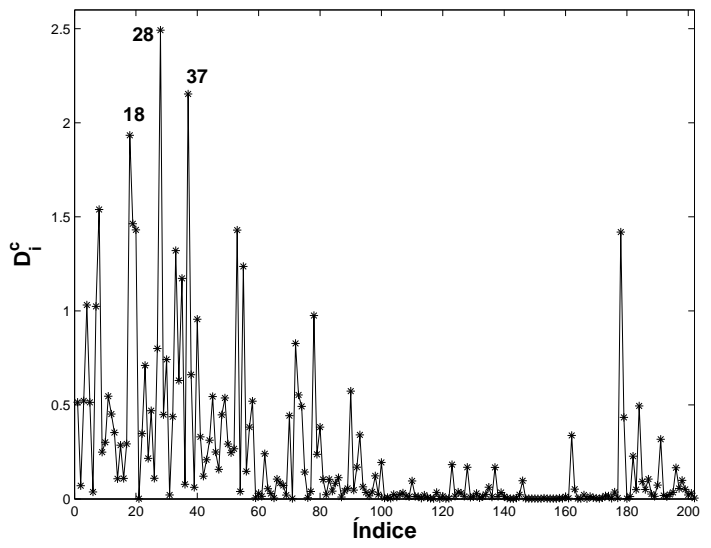

(b)

Figura 3.10: Conjunto de dados AIS. (a) Afastamento pela verossimilhança $L D_{i}^{c}$ e (b) distância de Cook $D_{i}^{c}$.

de confiança (Fig. 3.9b). Portanto, aliado ao gráfico de envelope simulado, percebe-se que o modelo t-normal assimétrico é mais indicado ao conjunto de dados.

Para esse conjunto de dados foi conduzido um estudo de influência local com interesse em $\boldsymbol{\theta}$, baseado em $M(0)$.Para todos os esquemas de perturbação, os indivíduos 11, 18, 37, 53 e 56 ultrapassaram o ponto de corte, como indicado na Figura 2.6. Nota-se também nos casos de diagnóstico de eliminação de casos, as medidas $D_{i}^{c}$ e $L D_{i}^{c}$ apresentadas nas Figuras 3.6 (a e b), respectivamente, indicam que os mesmos indivíduos parecem ser mais influentes.

Invariavelmente, são os pontos situados na cauda leve da distribuição (abaixo da reta estimada) os mais influentes na estimação dos parâmetros $\left(D_{i}^{c}\right)$. A medida de afastamento pela verossimilhança é a mais sensível de todas, tendo como pontos influentes aqueles abaixo e acima da reta.

As medidas de influência local detectaram como pontos potencialmente influentes aqueles situados abaixo da reta e também aqueles remotos do subespaço gerado pelas colunas de $\mathbf{X}$ (pontos de alavanca). Estes últimos contradizem em certo sentido as simulações realizadas, onde invariavelmente os pontos acima da reta (cauda pesada) é que são influentes sob essas perturbações.

O ponto (56) aparece possivelmente influente de acordo com todas as análises, com exceção de $D_{i}^{c}$, sendo este um ponto de alavanca. Uma possível explicação para isso pode ser o fato de que as variáveis ssf e bfat têm conjuntamente uma distribuição t-normal 


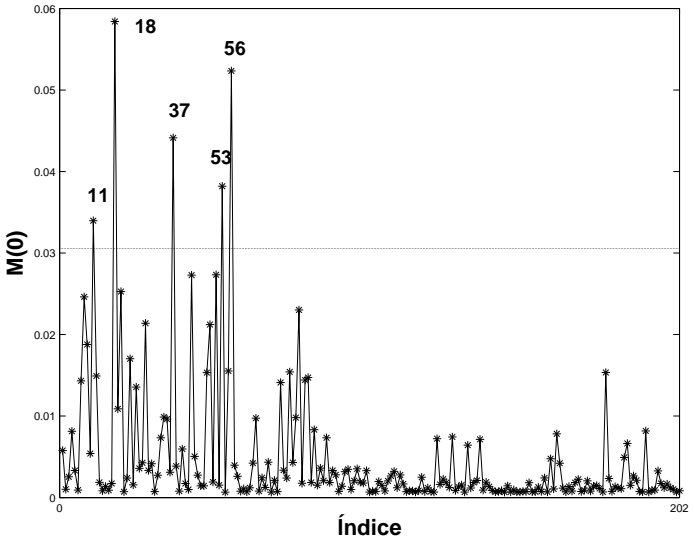

(a)

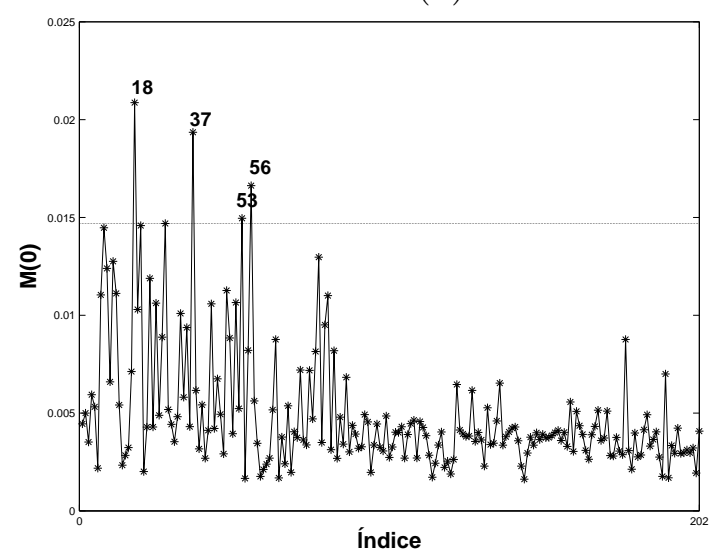

(c)

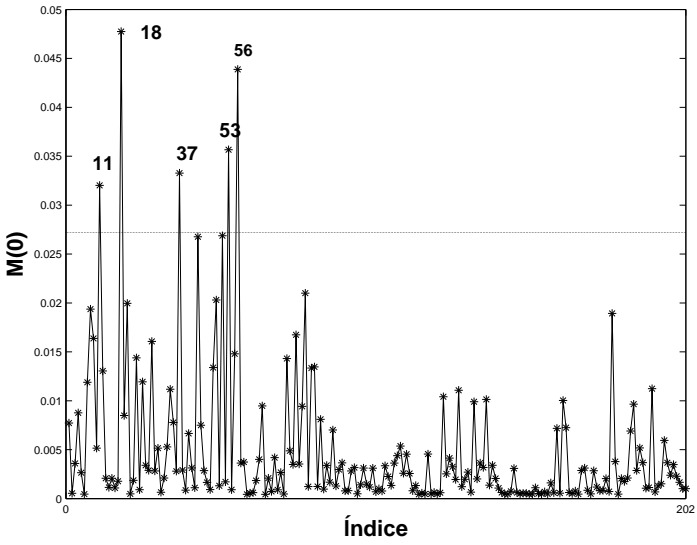

(b)

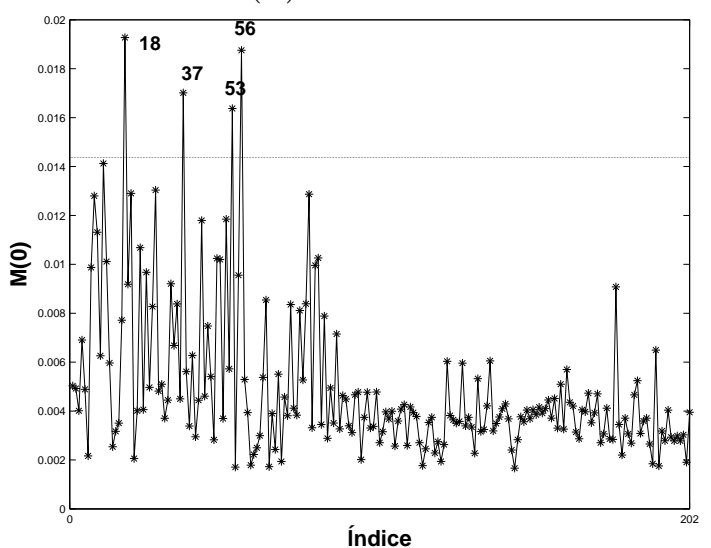

(d)

Figura 3.11: Gráficos de diagnóstico para o conjunto de dados AIS: (a) Perturbação de ponderação de casos, (b) perturbação no parâmetro de escala, (c) perturbação na variável explicativa e (d) perturbação na variável resposta. Linhas horizontais são os pontos de corte $\left(c^{*}=3\right)$.

assimétrica bivariada. Ainda, existe uma forte dependência linear entre as duas, sem ter pontos distantes acima ou abaixo da reta, mas sim na direção da mesma.

Neste capítulo não foi realizada a análise da matriz de alavancagem, devido à dificuldade de derivar a matriz $D_{\boldsymbol{\theta}}=\frac{\partial \boldsymbol{\mu}}{\partial \boldsymbol{\theta}^{\top}}$, desde que o valor esperado $\boldsymbol{\mu}=E[\mathbf{Y}]$ envolver uma expressão complicada. No entanto, no Capítulo 4, a matriz de alavancagem é desenvolvida e a análise de pontos de alavanca é realizada.

O modelo t-normal assimétrico, um modelo mais geral que o modelo normal, possui a característica de incorporar observações extremas na cauda pesada, o que o torna um modelo mais robusto. O próximo capítulo generaliza ainda mais a classe de distribuições assimétricas, 
utilizando o trabalho de Lange e Sinsheimer (1993), criando uma classe de distribuições de misturas de escalas normais assimétricas, englobando as distribuições normal, t-normal, slash, normal contaminada e exponencial potência assimétricas.

Ferreira, C. S. 


\section{Modelo de Misturas de Escala Normal Assimétrico}

\subsection{Introdução}

Misturas de escala da distribuição normal são freqüentemente usadas para procedimentos estatísticos de dados simétricos (Dempster et al., 1980; Little, 1988; Lange et al., 1989; Lange e Sinsheimer, 1993; entre outros). Nesse capítulo, define-se uma versão assimétrica dessas distribuições e são derivadas algumas de suas propriedades e inferência paramétrica. A principal virtude dos membros dessa família de distribuições é que as mesmas são fáceis de serem geradas por simulação e também fornecem algoritmos EM genuínos para estimação de máxima verossimilhança. Para respostas univariadas assimétricas, os algoritmos EM são desenvolvidos com ênfase nas distribuições t-normal, slash, normal contaminada e exponencial potência assimétricas. Resultados obtidos de conjuntos de dados reais e simulados são reportados ilustrando a utilidade da metodologia proposta. A conclusão principal em analisar um conjunto de dados previamente estudado é que os modelos até agora introduzidos claramente não são os mais adequados.

A mistura de escala da distribuição normal (Andrews e Mallows, 1974) fornece um grupo de distribuições com caudas pesadas que são freqüentemente usadas para inferência robusta de dados simétricos. A teoria e a aplicação (através de simulação ou experimentação) normalmente gera uma grande quantidade de conjuntos de dados que são assimétricos ou com caudas pesadas, por exemplo, dados de rendimento familiar (Azzalini et al., 2003) ou concentração de substâncias (Galea et al., 2003 e Lachos e Bolfarine, 2007). Assim, distribuições apropriadas para ajustar e simular esses dados assimétricos ou concentrados nas caudas se fazem necessárias. Uma nova família de distribuições que combine assimetria com caudas pesadas é desenvolvida. Mais ainda, essas distribuições se destacam porque 
têm uma representação estocástica que permite uma fácil implementação do algoritmo EM e também facilita o estudo de muitas das suas propriedades. A proposta desta distribuição generaliza estudos encontrados na maior parte em Lange e Sinsheimer (1993) (veja também Andrews e Mallows, 1974).

Pensando como Azzalini (1985) e Azzalini e Capitanio (2003), é natural construir distribuições univariadas e multivariadas que combinam assimetria com caudas pesadas. Por exemplo, podemos definir as distribuições $t$ de Student assimétricas (Sahu et al., 2003), as distribuições Cauchy assimétricas (Arnold Beaver, 2000), as distribuições Slash assimétricas (Wang e Genton, 2006), as distribuições t-slash assimétricas (Tan e Peng, 2006), as distribuições elípticas assimétricas (Azzalini e Capitanio, 1999; Branco e Dey, 2001; Sahu et al., 2003; Genton e Loperfido, 2005). Diferentemente das idéias acima, nesse capítulo, define-se uma nova família de distribuições assimétricas univariadas gerada pelo núcleo da normal (como função de assimetria) usando, por outro lado, distribuições simétricas da classe das distribuições com mistura de escala normais (Andrews e Mallows, 1974; Lange e Sinsheimer, 1993). Algumas de suas propriedades e inferência são estudadas e também discutidas aplicações a dados reais. Um aspecto interessante e simplificador da família é que a implementação do algoritmo EM é facilitada pelo fato de os passos E serem exatamente como na família de modelos normal/independente (NI), proposta em Lange e Sinsheimer (1993). Além disso, o passo M envolve expressões fechadas que facilitam a implementação do algoritmo e também conclui-se que a matriz de informação tem uma parte em comum com todos os elementos da família.

\subsection{Distribuições de Misturas de Escala Normal}

A classe simétrica das distribuições de mistura de escala normal (MEN) inclui distribuições tais como normal, t de Student, slash, exponencial potência, normal contaminada, entre outras. Todas essas distribuições têm caudas mais pesadas que a distribuição normal. Diz-se que uma variável aleatória $Y$ tem uma distribuição MEN com parâmetro de locação $\mu \in \mathbb{R}$ e parâmetro de escala $\sigma^{2}$ se sua função densidade de probabilidade (fdp) assume a forma

$$
f(y)=\int_{0}^{\infty} \phi\left(y \mid \mu, \kappa(u) \sigma^{2}\right) d H(u ; \boldsymbol{\tau})
$$

Ferreira, C. S. 
onde $H(. ; \boldsymbol{\tau})$ é a função distribuição acumulada (fda) de uma variável aleatória positiva $U$ indexada pelo vetor de parâmetros $\boldsymbol{\tau}$ (suposto conhecido) e $\kappa($.$) é uma função estritamente$ positiva. Para uma variável aleatória com fdp como em (4.1), será utilizada a notação $Y \sim \operatorname{MEN}\left(\mu, \sigma^{2} ; H\right)$. Mais ainda, quando $\mu=0$ e $\sigma^{2}=1$, denota-se $Y \sim \operatorname{MEN}(H)$. Sua representação estocástica é dada por

$$
Y=\mu+\kappa^{1 / 2}(U) Z
$$

onde $Z \sim \mathrm{N}\left(0, \sigma^{2}\right)$ e $U$ é uma variável aleatória positiva independente de $Z$. Alguns exemplos de distribuições MEN são descritos abaixo (ver Lange e Sinsheimer, 1993). Para essa família, as propriedades da distribuição da distância Mahalanobis ao quadrado

$$
D=\frac{(Y-\mu)^{2}}{\sigma^{2}}
$$

são descritas, pelo fato de serem extremamente úteis para testar bondade de ajuste e detectar a presença de pontos extremos.

\subsubsection{Exemplos de distribuições MEN}

- A distribuição $t$ de Student generalizada com $\nu>0$ graus de liberdade, $\gamma>0, Y \sim$ $G t\left(\mu, \sigma^{2} ; \nu, \gamma\right)$.

O uso da distribuição $t$ de Student como uma alternativa à distribuição normal tem sido freqüentemente sugerida na literatura. Por exemplo, foi usada por Little (1988) e Lange et al. (1989) para modelagem robusta. Neste caso, $Y$ tem função de densidade dada por

$$
f(y)=\frac{1}{\sigma \sqrt{\gamma \pi}} \frac{\Gamma((\nu+1) / 2)}{\Gamma\left(\frac{\nu}{2}\right)}\left(1+\frac{d}{\gamma}\right)^{-\left(\frac{\nu+1}{2}\right)},
$$

com $d=(y-\mu)^{2} / \sigma^{2}$. Neste caso, $\kappa(U)=1 / U$ e $U \sim \operatorname{Gama}(\nu / 2, \gamma / 2)$, com densidade

$$
h(u ; \nu, \gamma)=\frac{(\gamma / 2)^{\nu / 2}}{\Gamma(\nu / 2)} u^{\nu / 2-1} e^{-\gamma u / 2}
$$

com $E\left[U^{-m}\right]=\frac{(\gamma / 2)^{m} \Gamma(\nu / 2-m)}{\Gamma(\nu / 2)}$, for $m<\nu / 2$. Se $\gamma=1$ e $\nu \uparrow \infty$, tem-se que $Y \stackrel{\mathrm{D}}{\rightarrow} N A\left(\mu, \sigma^{2}, \lambda\right)$. Foi mostrado por Lange e Sinsheimer (1993) que, para $\nu=\gamma$, a distância Mahalanobis ao quadrado $D$ tem distribuição $F$. Neste caso, uma mani- 
pulação algébrica simples leva a

$$
\frac{\nu}{\gamma} D=\frac{\nu}{\gamma} \frac{(Y-\mu)^{2}}{\sigma^{2}} \sim F_{1, \nu}
$$

- A distribuição slash, $Y \sim S L\left(\mu, \sigma^{2} ; \nu\right)$, com parâmetro de forma $\nu>0$.

Essa distribuição apresenta caudas mais pesadas que aquelas da distribuição normal e inclui o caso da normal quando $\nu \uparrow \infty$. Sua fdp é dada por

$$
f(y)=\frac{\nu}{\sqrt{2 \pi} \sigma} \int_{0}^{1} u^{\nu-1 / 2} e^{-u d / 2} d u
$$

Aqui temos que $\kappa(U)=1 / U$ e $U$ com densidade

$$
h(u ; \nu)=\nu u^{\nu-1} \mathbf{I}_{(0,1)}(u),
$$

com $E\left[U^{-m}\right]=\frac{\nu}{\nu-m}$, for $m<\nu$, onde a notação $\mathbf{I}_{(A)}$ é a função indicadora do conjunto A. A distância de Mahalanobis ao quadrado tem função distribuição dada por

$$
\operatorname{Pr}(D \leq r)=\operatorname{Pr}\left(\chi^{2} \leq r\right)-\frac{2^{\nu} \Gamma(\nu+1 / 2)}{r^{\nu} \sqrt{\pi}} \operatorname{Pr}\left(\chi_{2 \nu+1}^{2} \leq r\right)
$$

- $A$ distribuição normal contaminada, $Y \sim N C\left(\mu, \sigma^{2} ; \nu, \gamma\right), 0 \leq \nu \leq 1,0<\gamma \leq 1$ (Little, 1988).

Essa distribuição pode também ser aplicada para modelar dados simétricos com pontos extremos. O parâmetro $\nu$ representa a porcentagem de outliers, enquanto $\gamma$ pode ser interpretado como um fator de escala. Sua fpd é dada por

$$
f(y)=\nu \phi\left(y \mid \mu, \sigma^{2} / \gamma\right)+(1-\nu) \phi\left(y \mid \mu, \sigma^{2}\right)
$$

Nesse caso, $\kappa(U)=1 / U$ e a fdp $h(u ; \nu, \gamma)$ dada por

$$
h(u ; \nu, \gamma)=\nu \mathbb{I}_{(u=\gamma)}+(1-\nu) \mathbb{I}_{(u=1)}, \quad \boldsymbol{\tau}=(\nu, \gamma)^{\top} .
$$

Essa distribuição inclui o caso normal quando $\gamma=1$ e $\nu=1$ ou $\nu=1 / 2$. Claramente, $E\left[U^{-m}\right]=\nu / \gamma^{m}+1-\nu, \mathrm{e}$

$$
\operatorname{Pr}(D \leq r)=\nu \operatorname{Pr}\left(\chi^{2} \leq \gamma r\right)+(1-\nu) \operatorname{Pr}\left(\chi^{2} \leq r\right)
$$

- A distribuição exponencial potência, $Y \sim E P\left(\mu, \sigma^{2} ; \nu\right)$, com parâmetro de forma $0<$ 
$\nu \leq 1$

Sua fdp é dada por

$$
f(y)=\frac{\nu}{2^{\frac{1}{2 \nu}} \sigma \Gamma\left(\frac{1}{2 \nu}\right)} e^{-d^{\nu} / 2} .
$$

Quando $\nu=1$, tem-se a densidade da distribuição normal. Aqui $U$ tem densidade positiva estável $S^{P}(u \mid \nu)$ (Branco e Dey, 2001).

De Lange e Sinsheimer (1993), a distância Mahalanobis ao quadrado tem fda dada por

$$
\operatorname{Pr}(D \leq r)=\frac{r^{1 / 2} G\left(\frac{1}{2 \nu}, r^{\nu} / 2\right)}{\Gamma\left(\frac{1}{2 \nu}\right) 2^{\frac{1}{2 \nu}}},
$$

onde $G(\beta, s)=\int_{0}^{s} u^{\beta-1} e^{-u} d u$ é a função Gama incompleta.

\subsection{Distribuições de Misturas de Escala Normal As- simétricas}

Nesta seção, são definidas as distribuições de mistura de escala normais assimétricas (MENA) univariadas generalizando a família MEN estudando algumas de suas propriedades. Também será mostrado que as distribuições de transformações lineares pertencem à mesma classe.

Definição 4.1. Uma variável aleatória $Y$ segue uma distribuição na classe MENA com parâmetro de locação $\mu \in \mathbb{R}$, parâmetro de escala $\sigma^{2}$ e parâmetro de assimetria $\lambda \in \mathbb{R}$, se sua fdp for dada por

$$
f(y)=2 \int_{0}^{+\infty} \phi\left(y \mid \mu, \sigma^{2} \kappa(u)\right) \Phi\left(\lambda \frac{y-\mu}{\sigma}\right) d H(u ; \boldsymbol{\tau}), y \in \mathbb{R}
$$

onde $U$ é uma variável aleatória positiva com $f d a H(u ; \tau)$ e $\kappa($.$) é uma função estritamente$ positiva. Neste caso, denota-se $Y \sim \operatorname{MENA}\left(\mu, \sigma^{2}, \lambda ; H\right)$. Se $\mu=0$ e $\sigma^{2}=1$, tem-se uma distribuição MENA padrão, denotada por $M E N A(\lambda ; H)$.

Claramente, de (4.7), quando $\lambda=0$ tem-se a distribuição MEN correspondente definida em (4.1).

Para uma variável aleatória da família MENA, a representação estocástica dada abaixo pode ser usada para simular rapidamente pseudo-realizações de $y$ e também estudar muitas 
de suas propriedades.

Proposição 4.1. Seja $Y \sim \operatorname{MENA}\left(\mu, \sigma^{2}, \lambda ; H\right)$. Então, uma representação estocástica é dada por

$$
\begin{aligned}
Y \mid U=u & \sim N A\left(\mu, \sigma^{2} \kappa(u), \lambda \sqrt{\kappa(u)}\right) \\
U & \sim H(u ; \boldsymbol{\tau}) .
\end{aligned}
$$

Demonstração. De (4.7), a função densidade (conjunta) de $(Y, U)$ é dada por

$$
g(y, u)=2 \phi\left(y \mid \mu, \sigma^{2} \kappa(u)\right) \Phi(\lambda(y-\mu) / \sigma) f_{U}(u)
$$

Fornecendo $g(y, u)=g(y \mid u) f_{U}(u)$, então

$$
\begin{aligned}
g(y \mid u) & =2 \phi\left(y \mid \mu, \sigma^{2} \kappa(u)\right) \Phi\left(\lambda \frac{(y-\mu)}{\sigma}\right) \\
& =2 \phi\left(y \mid \mu, \sigma^{2} \kappa(u)\right) \Phi\left(\lambda \sqrt{\kappa(u)} \frac{(y-\mu)}{\sigma \sqrt{\kappa(u)}}\right)
\end{aligned}
$$

e então $Y \mid U=u \sim N A\left(\mu, \sigma^{2} \kappa(u), \lambda \sqrt{\kappa(u)}\right)$.

Observação 4.1. Em outras palavras, pode-se gerar de uma distribuição independente normal assimétrica, procedendo em dois passos, gerando primeiro de uma distribuição de $U$ e depois de uma distribuição condicional $Y \mid U$ usando, por exemplo, a representação estocástica dada em (2.3) no Cap. 2.

Na próxima proposição, é fornecida uma expressão geral para a função geradora de momentos (fgm) de uma variável aleatória $M E N A$.

Proposição 4.2. Seja $Y \sim \operatorname{MENA}\left(\mu, \sigma^{2}, \lambda ; H\right)$. Então

$$
M_{Y}(r)=E\left[e^{r Y}\right]=\int_{0}^{\infty} 2 e^{r \mu+\frac{r^{2}}{2} \kappa(u) \sigma^{2}} \Phi\left(\frac{\sigma \lambda \kappa(u)}{\sqrt{1+\lambda^{2} \kappa(u)}} r\right) d H(u), \quad r \in \mathbb{R} .
$$

Demonstração. Da Proposição 4.1, tem-se que $Y \mid U=u \sim N A\left(\mu, \sigma^{2} \kappa(u), \lambda \sqrt{\kappa(u)}\right)$. Mais ainda, de propriedades bem conhecidas de esperança condicional, segue que $M_{Y}(r)=E_{U}\left[E\left[e^{r Y} \mid U\right]\right]$. De Gupta e Huang (2002), $M_{Z}(z)=2 \exp \left(\frac{z^{2}}{2}\right) \Phi\left(\frac{\lambda z}{\sqrt{1+\lambda^{2}}}\right)$, onde 
$Z \sim N A(\lambda)$. Então, dado que $M_{a+b Y}(r)=e^{a r} M_{Y}(b r)$, obtém-se

$$
M_{Y \mid U=u}(r)=2 e^{r \mu+\frac{r^{2}}{2} \kappa(u) \sigma^{2}} \Phi\left(\frac{\sigma \lambda \kappa(u)}{\sqrt{1+\lambda^{2} \kappa(u)}} r\right) .
$$

Na proposição seguinte são apresentadas a média e a variância de uma variável aleatória MENA.

Proposição 4.3. Suponha que $Y \sim M E N A\left(\mu, \sigma^{2}, \lambda ; H\right)$. Então,

a)

$$
E[Y]=\mu+c \sigma \lambda E_{U}\left[\frac{\kappa(U)}{\sqrt{1+\lambda^{2} \kappa(U)}}\right] e
$$

b)

$$
\operatorname{Var}[Y]=\sigma^{2}\left[E_{U}[\kappa(U)]-c^{2} \lambda^{2} E_{U}^{2}\left[\frac{\kappa(U)}{\sqrt{1+\lambda^{2} \kappa(U)}}\right]\right]
$$

onde $c=\sqrt{\frac{2}{\pi}}$.

Observação 4.2. Se $\kappa(U)=1$, então $E[Y]=\mu+c \sigma \delta$ e $\operatorname{Var}[Y]=\sigma^{2}\left(1-c^{2} \delta^{2}\right)$, são os mesmos valores de média e variância de uma variável aleatória normal assimétrica.

Outra propriedade importante da classe MENA, uma extensão de um resultado apresentado em Wang et al. (2005), é apresentada a seguir.

Proposição 4.4. Se $Y \sim M E N A\left(\mu, \sigma^{2}, \lambda ; H\right)$, então para qualquer função par $\xi$, a distribuição de $\xi(Y-\mu)$ não depende de $\lambda$ e tem a mesma distribuição que $\xi(X-\mu)$, onde $X \sim M E N\left(\mu, \sigma^{2} ; H\right)$. Assim, $(Y-\mu)^{2} e(X-\mu)^{2}$ são identicamente distribuídos.

Demonstração. Considerando $X \sim \operatorname{MEN}\left(0, \sigma^{2} ; H\right), f_{X}\left(y-\mu \mid 0, \sigma^{2} ; H\right)$ a densidade MEN 
como em (4.1), então

$$
\begin{aligned}
M_{\xi(Y-\mu)}(t)= & \int_{\mathbb{R}} 2 e^{\xi(y-\mu) t} f_{X}\left(y-\mu \mid 0, \sigma^{2} ; H\right) \Phi\left[\lambda \frac{(y-\mu)}{\sigma}\right] d y \\
= & \int_{\mathbb{R}^{+}} 2 e^{\xi(x) t} f_{X}\left(x \mid 0, \sigma^{2} ; H\right) \Phi\left(\lambda \frac{x}{\sigma}\right) d x \\
& +\int_{\mathbb{R}^{-}} 2 e^{\xi(x) t} f_{X}\left(x \mid 0, \sigma^{2} ; H\right)\left[1-\Phi\left(-\lambda \frac{x}{\sigma}\right)\right] d x \\
= & \int_{\mathbb{R}^{+}} 2 e^{\xi(x) t} f_{X}\left(x \mid 0, \sigma^{2} ; H\right)\left[\Phi\left(\lambda \frac{x}{\sigma}\right)+1-\Phi\left(\lambda \frac{x}{\sigma}\right)\right] d x \\
= & \int_{\mathbb{R}^{2}} e^{\xi(x) t} f_{Y} X\left(x \mid 0, \sigma^{2} ; H\right) d x \\
= & M_{\xi(X)}(t) .
\end{aligned}
$$

Com uma conseqüência da Preposição 4.4, tem-se os seguintes resultados interessantes.

Corolário 4.1. Seja $Y \sim M E N A\left(\mu, \sigma^{2}, \lambda ; H\right)$. Então, a forma quadrática

$$
D_{\lambda}=\frac{(Y-\mu)^{2}}{\sigma^{2}}
$$

tem a mesma distribuição que $D=\frac{(X-\mu)^{2}}{\sigma^{2}}$, onde $X \sim M E N\left(\mu, \sigma^{2} ; H\right)$.

O resultado do corolário 4.1 é interessante porque permite checar o modelo na prática. De Lange e Sinsheimer (1993) e do Corolário 4.1, tem-se que

$$
E\left[D_{\lambda}^{m}\right]=E\left[D^{m}\right]=\frac{2^{m} \Gamma(m+1 / 2)}{\sqrt{\pi}} E\left[\kappa(U)^{m}\right], \quad m>0 .
$$

Na próxima proposição é mostrado que uma variável aleatória MENA é invariante sob transformações lineares. Esse resultado é um resumo da seguinte proposição:

Proposição 4.5. Seja $Y \sim \operatorname{MENA}\left(\mu, \sigma^{2}, \lambda ; H\right)$. Então para todo valor fixo $b \in \mathbb{R}$,

$$
V=a+b Y \sim M E N A\left(a+b \mu, b^{2} \sigma^{2}, \operatorname{sinal}(b) \lambda ; H\right) .
$$

Demonstração. A prova segue diretamente da Proposição 4.2, visto que $M_{a+b y}(r)=e^{a r} M_{Y}(b r)$. 
Usando (4.10), quando $a=0$ e $b=-1$, tem-se a seguinte propriedade adicional de uma variável aleatória $M E N A$.

Corolário 4.2. Seja $Y \sim M E N A\left(\mu, \sigma^{2}, \lambda ; H\right)$. Então, $-Y \sim M E N A\left(-\mu, \sigma^{2},-\lambda ; H\right)$.

\subsubsection{Exemplos de distribuições MENA}

- Distribuição t de Student generalizada normal assimétrica, com $\nu>0$ graus de liberdade, $\gamma>0$, denotada $Y \sim \operatorname{Gt} A\left(\mu, \sigma^{2}, \lambda ; \nu, \gamma\right)$. Considerando $U \sim \operatorname{Gama}(\nu / 2, \gamma / 2)$, $\kappa(U)=1 / U, Y$ tem a fdp

$$
f(y)=2 \frac{1}{\sigma \sqrt{\gamma \pi}} \frac{\Gamma((\nu+1) / 2)}{\Gamma\left(\frac{\nu}{2}\right)}\left(1+\frac{d}{\gamma}\right)^{-\left(\frac{\nu+1}{2}\right)} \Phi\left[\lambda \frac{(y-\mu)}{\sigma}\right], y \in \mathbb{R} .
$$

Para $\gamma=\nu$, tem-se uma distribuição t-normal assimétrica. Outro caso particular da distribuição t-normal assimétrica é a distribuição Cauchy normal assimétrica, que segue quando $\nu=\gamma=1$. Também, quando $\nu \uparrow \infty$, tem-se a distribuição normal assimétrica como caso limite. A média e a variância de $Y \sim G t A\left(\mu, \sigma^{2}, \lambda ; \nu, \gamma\right)$ são dadas por $(\nu>2)$

$$
\begin{aligned}
E[Y] & =\mu+c \sigma \lambda(\gamma / 2)^{1 / 2} \frac{\Gamma((\nu-1) / 2)}{\Gamma(\nu / 2)} E_{V}\left[\left(V+\lambda^{2}\right)^{-1 / 2}\right] \mathrm{e} \\
\operatorname{Var}[Y] & =\sigma^{2}\left[\frac{\gamma}{\nu-2}-\frac{c^{2} \lambda^{2} \gamma}{2}\left(\frac{\Gamma((\nu-1) / 2)}{\Gamma(\nu / 2)}\right)^{2} E_{V}^{2}\left[\left(V+\lambda^{2}\right)^{-1 / 2}\right]\right],
\end{aligned}
$$

onde $V \sim \operatorname{Gama}\left(\frac{\nu-1}{2}, \frac{\gamma}{2}\right)$. Os valores esperados são calculados numericamente, através de integração de Monte Carlo. Se $\gamma=\nu$, os valores de (4.12) e (4.13) coincidem com os valores dados para a distribuição t-normal assimétrica em (3.3).

- Distribuição slash assimétrica, com parâmetro de forma $\nu>0$, denotada por $S L A\left(\mu, \sigma^{2}, \lambda ; \nu\right)$. Com $h(u ; \nu)$ como em $(4.5)$ e $\kappa(U)=1 / U$, tem-se que

$$
f(y)=2 \nu \Phi\left(\lambda \frac{y-\mu}{\sigma}\right) \int_{0}^{1} u^{\nu-1} \phi\left(y \mid \mu, \frac{\sigma^{2}}{u}\right) d u, \quad y \in \mathbb{R} .
$$

A distribuição slash assimétrica reduz-se à distribuição normal assimétrica quando 
$\nu \uparrow \infty$. A média e a variância são dadas por $(\nu>1)$

$$
\begin{aligned}
E[Y] & =\mu+\frac{c \sigma \lambda \nu}{\nu-1 / 2} E_{V}\left[\left(V+\lambda^{2}\right)^{-1 / 2}\right] \mathrm{e} \\
\operatorname{Var}[Y] & =\sigma^{2}\left(\frac{\nu}{\nu-1}-\frac{c^{2} \lambda^{2} \nu^{2}}{(\nu-1 / 2)^{2}} E_{V}^{2}\left[\left(V+\lambda^{2}\right)^{-1 / 2}\right]\right),
\end{aligned}
$$

onde $V \sim \operatorname{Beta}(1, \nu-1 / 2)$.

- Distribuição normal contaminada assimétrica, denotada por $N C A\left(\mu, \sigma^{2}, \lambda ; \nu, \gamma\right), 0 \leq$ $\nu \leq 1,0<\gamma \leq 1$. Sendo $h(u ; \nu)$ como em (4.6), segue que

$$
\begin{aligned}
f(y)= & 2\left\{\nu \phi\left(y \mid \mu, \frac{\sigma^{2}}{\gamma}\right) \Phi\left(\lambda \frac{y-\mu}{\sigma}\right)\right. \\
& \left.+(1-\nu) \phi\left(y \mid \mu, \sigma^{2}\right) \Phi\left(\lambda \frac{y-\mu}{\sigma}\right)\right\}, y \in \mathbb{R} .
\end{aligned}
$$

A distribuição normal contaminada assimétrica reduz-se à distribuição normal assimétrica quando $\gamma=1$ e $\nu=1$ ou $\nu=1 / 2$. A média e a variância são dadas por

$$
\begin{gathered}
E[Y]=\mu+c \sigma \lambda\left(\frac{\nu}{\left(\gamma\left(\gamma+\lambda^{2}\right)\right)^{1 / 2}}+\frac{1-\gamma}{\left(1+\lambda^{2}\right)^{1 / 2}}\right) \text { e } \\
\operatorname{Var}[Y]=\sigma^{2}\left[\frac{\nu}{\gamma}+1-\nu-c^{2} \lambda^{2}\left(\frac{\nu}{\left(\gamma\left(\gamma+\lambda^{2}\right)\right)^{1 / 2}}+\frac{1-\gamma}{\left(1+\lambda^{2}\right)^{1 / 2}}\right)^{2}\right] .
\end{gathered}
$$

- Distribuição exponencial potência assimétrica, denotada por $Y \sim \operatorname{EPA}\left(\mu, \sigma^{2}, \lambda ; \nu\right)$, com parâmetro $0<\nu \leq 1$.

Sua fdp é dada por

$$
f(y)=2 \frac{\nu}{2^{\frac{1}{2 \nu}} \sigma \Gamma\left(\frac{1}{2 \nu}\right)} e^{-d^{\nu} / 2} \Phi\left(\lambda \frac{y-\mu}{\sigma}\right) .
$$

A distribuição exponencial potência assimétrica reduz-se à distribuição normal assimétrica quando $\nu=1$. Nesse caso, $\kappa(u)$ não tem forma explícita.

A Figura 4.1 apresenta a função densidade da distribuição normal padrão assimétrica $N A(3)$, junto com as densidades padrão das distribuições $G t A(3 ; 2,2), S L A(3 ; 0.5)$, $N C A(3 ; 0.9,0.1)$ e $\operatorname{EPA}(3 ; 0.5)$. Os valores das densidades são transformados de forma que tenham os mesmos valores na origem, para efeito de comparação das curvas. Note que as cinco densidades são assimétricas positivas, e que as distribuições normal contaminada 
assimétrica, exponencial potência assimétrica, slash assimétrica e t-normal assimétrica têm caudas muito mais pesadas do que a normal assimétrica. Note que todas elas representam situações extremas, pelo menos no caso simétrico, por exemplo, a slash com $\nu=0.5$ não tem o primeiro momento finito.

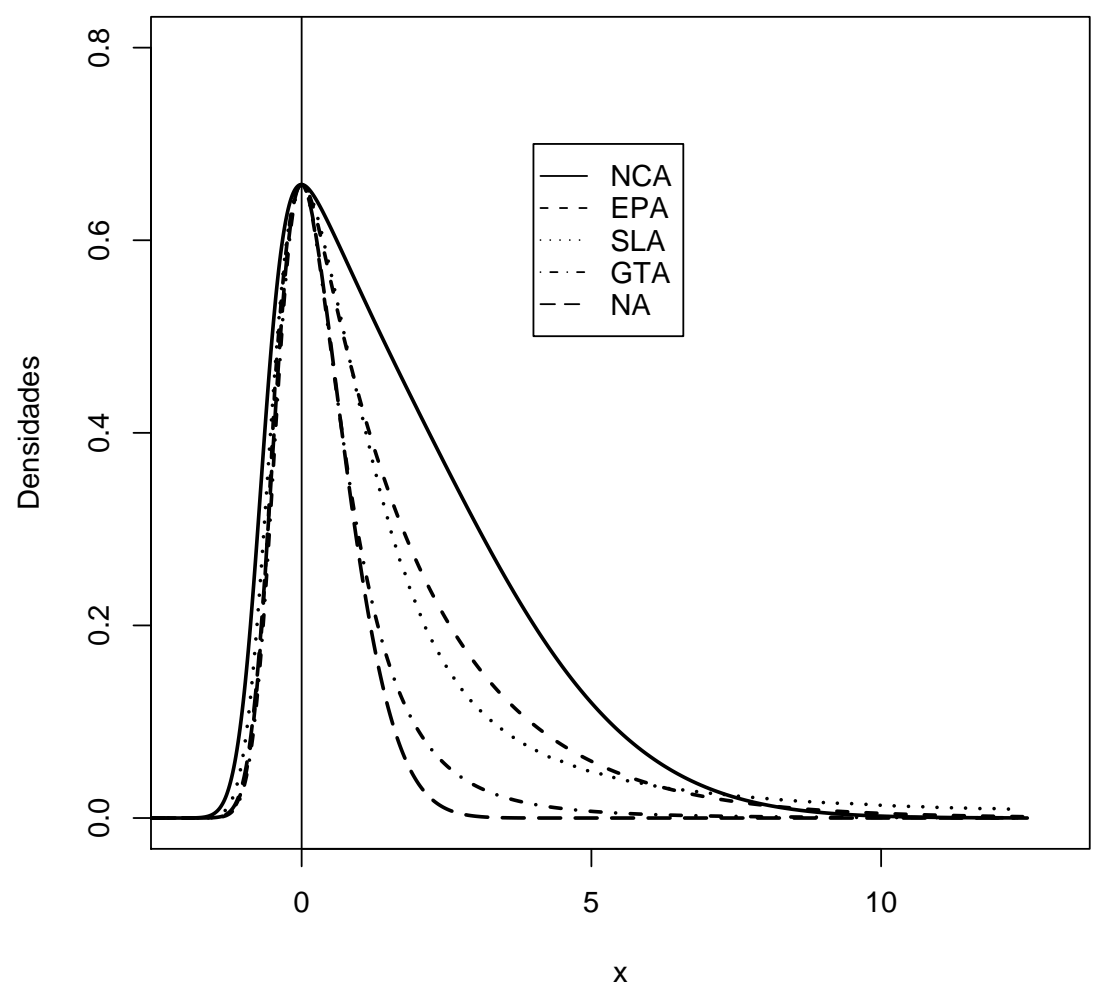

Figura 4.1: Funções de densidade das distribuições normal $N A(3)$, t-normal generalizada $G t A(3 ; 2,2)$, slash $S L A(3 ; 0.5)$, normal contaminada $N C A(3 ; 0.9,0.1)$ e exponencial potência EPA(3;0.5) assimétricas.

Em seguida é proposto um algoritmo EM para obter a estimativa MV do vetor de parâmetros $\boldsymbol{\theta}$. A metodologia proposta para a classe de modelos tratada aqui não existe na literatura. Um feito especial, no entanto, é que o passo E é como em Lange e Sinsheimer (1993). Mais ainda, estudos relacionados com influência local para dados incompletos (Zhu e Lee, 2001) podem ser facilmente estendidos.

Ferreira, C. S. 


\subsection{Modelos de Regressão MENA e o Algoritmo EM}

Suponha que se tenha observações independentes de $n$ indivíduos, denotadas por $Y_{1}, \ldots, Y_{n}$, onde $Y_{i} \sim \operatorname{MENA}\left(\mu_{i}, \sigma^{2}, \lambda ; H\right), i=1, \ldots, n$. Associado ao indivíduo $i$ assume-se um vetor $p \times 1$ de covariáveis conhecido $\mathbf{x}_{i}$, o qual será utilizado para especificar a predição linear $\mu_{i}=\mathbf{x}_{i}^{\top} \boldsymbol{\beta}$, onde $\boldsymbol{\beta}$ é um vetor $p$-dimensional de coeficientes de regressão desconhecidos. Então, relacionando os dois conjuntos de variáveis considera-se

$$
\begin{aligned}
y_{i} & =\beta_{0}+\sum_{k=1}^{p} x_{i k} \beta_{k}+\sigma \varepsilon_{i}, \quad i=1, \ldots, n, \\
& =\mathbf{x}_{i}^{\top} \boldsymbol{\beta}+\varepsilon_{i}, \quad \varepsilon_{i} \sim \operatorname{MENA}(\lambda ; H) .
\end{aligned}
$$

Note que $E\left[\varepsilon_{i}\right]=\sqrt{\frac{2}{\pi}} \lambda E_{U}\left[\frac{\kappa(U)}{\sqrt{1+\lambda^{2} \kappa(U)}}\right] \neq 0$, para $\lambda \neq 0$. Em termos de previsão para $Y$, geralmente considera-se $\widehat{Y}_{i}=\widehat{\beta}_{0}+\sum_{k=1}^{p} x_{i k} \widehat{\beta}_{k}$ como preditor de $Y_{i} \mid \mathbf{x}_{i}$. Uma forma de corrigir esta distorção é considerar $\widehat{Y}_{i}=\widehat{\beta}_{0}+\sum_{k=1}^{p} x_{i k} \widehat{\beta}_{k}+\sqrt{\frac{2}{\pi}} \widehat{\sigma} \widehat{\lambda} E_{U}\left[\frac{\kappa(U)}{\sqrt{1+\widehat{\lambda}^{2} \kappa(U)}}\right]$ como preditor de $Y_{i} \mid \mathbf{x}_{i}$. Outra possibilidade é considerar o modelo centrado (ver Freitas, 2005).

Assim, de acordo com a expressão (C.1) no Apêndice C, a função de log-verossimilhança de dados observados de $\boldsymbol{\theta}=\left(\boldsymbol{\beta}^{\top}, \sigma^{2}, \lambda\right)^{\top}$ é dada por

$$
\ell(\boldsymbol{\theta})=\sum_{i=1}^{n} \log \left[2 \int_{0}^{+\infty} \int_{0}^{+\infty} \phi\left(y_{i} \mid \mathbf{x}_{i}^{\top} \boldsymbol{\beta}, \sigma^{2} \kappa\left(u_{i}\right)\right) \phi\left(t_{i} \mid \lambda\left(y_{i}-\mathbf{x}_{i}^{\top} \boldsymbol{\beta}\right), \sigma^{2}\right) h\left(u_{i} ; \boldsymbol{\tau}\right) d t_{i} d u_{i}\right] .
$$

Não seria fácil encontrar a estimativa MV do vetor de parâmetros $\boldsymbol{\theta}$ maximizando diretamente a função log-verossimilhança. Assim, é preferível implementar o algoritmo EM.

Note que, usando (4.8), o modelo definido acima pode ser escrito como

$$
\begin{aligned}
Y_{i} \mid T_{i}=t_{i}, U_{i}= & u_{i}, \stackrel{\stackrel{\text { ind }}{\sim}}{\sim} N\left(\mathbf{x}_{i}^{\top} \boldsymbol{\beta}+\sigma \lambda \frac{\kappa\left(u_{i}\right)}{\sqrt{1+\lambda^{2} \kappa\left(u_{i}\right)}} t_{i}, \frac{\sigma^{2} \kappa\left(u_{i}\right)}{1+\lambda^{2} \kappa\left(u_{i}\right)}\right), \\
U_{i} & \stackrel{\text { iid }}{\sim} H\left(u_{i} ; \boldsymbol{\tau}\right) \mathrm{e} \\
T_{i} & \stackrel{\mathrm{iid}}{\sim} N T(0,1), \quad i=1, \ldots, n,
\end{aligned}
$$

todos independentes, onde $N T(0,1)$ denota a distribuição normal truncada padrão. Assumese que o vetor de parâmetros $\boldsymbol{\tau}$ é conhecido. Em aplicações o valor ótimo de $\boldsymbol{\tau}$ pode ser escolhido usando a verossimilhança perfilada e o critério de informação de Schwarz (ver 
Lange et al., 1989).

Sejam $\mathbf{y}=\left(y_{1}, \ldots, y_{n}\right)^{\top}, \mathbf{u}=\left(u_{1}, \ldots, u_{n}\right)^{\top}$ e $\mathbf{t}=\left(t_{1}, \ldots, t_{n}\right)^{\top}$ e tratando $\mathbf{u}$ e $\mathbf{t}$ como faltantes, segue que a função log-verossimilhança completa associada a $\mathbf{y}_{c}=\left(\mathbf{y}^{\top}, \mathbf{u}^{\top}, \mathbf{t}^{\top}\right)^{\top}$ é dada por

$$
\ell_{c}\left(\boldsymbol{\theta} \mid \mathbf{y}_{c}\right) \propto-n \log \sigma^{2}-\frac{1}{2 \sigma^{2}} \sum_{i=1}^{n} \frac{\left(y_{i}-\mathbf{x}_{i}^{\top} \boldsymbol{\beta}\right)^{2}}{\kappa\left(u_{i}\right)}-\frac{1}{2 \sigma^{2}} \sum_{i=1}^{n}\left[t_{i}-\lambda\left(y_{i}-\mathbf{x}_{i}^{\top} \boldsymbol{\beta}\right)\right]^{2}
$$

Dado que $T_{i} \mid y_{i} \sim N T\left(\lambda\left(y_{i}-\mathbf{x}_{i}^{\top} \boldsymbol{\beta}\right), \sigma^{2}\right) \mathbb{I}_{(0, \infty)}\left(t_{i}\right)$ e sendo $\widehat{t}_{i}=\mathrm{E}\left[T_{i} \mid \boldsymbol{\theta}=\widehat{\boldsymbol{\theta}}, y_{i}\right], \widehat{t}^{2}{ }_{i}=$ $\mathrm{E}\left[T_{i}^{2} \mid \boldsymbol{\theta}=\widehat{\boldsymbol{\theta}}, y_{i}\right]$ e $\widehat{\kappa}_{i}=\mathrm{E}\left[\kappa^{-1}\left(U_{i}\right) \mid \boldsymbol{\theta}=\widehat{\boldsymbol{\theta}}, y_{i}\right]$ obtém-se, usando os momentos da distribuição normal truncada,

$$
\widehat{t_{i}}=\widehat{\lambda} \widehat{\eta}_{i}+\widehat{\sigma} W_{\Phi}\left(\frac{\widehat{\lambda} \widehat{\eta}_{i}}{\widehat{\sigma}}\right) \quad \text { e } \widehat{t}^{2}{ }_{i}=\widehat{\lambda}^{2} \widehat{\eta}_{i}^{2}+\widehat{\sigma}^{2}+\widehat{\lambda} \widehat{\sigma} \widehat{\eta}_{i} W_{\Phi}\left(\frac{\widehat{\lambda} \widehat{\eta}_{i}}{\widehat{\sigma}}\right)
$$

onde $W_{\Phi}(u)=\phi_{1}(u) / \Phi(u)$ e $\widehat{\eta}_{i}=y_{i}-\mathbf{x}_{i}^{\top} \widehat{\boldsymbol{\beta}}, i=1, \ldots, n$. Para as distribuições discutidas neste capítulo, $\widehat{\kappa}_{i}$ são dados pelas equações (4.26)-(4.29).

Denote por $\boldsymbol{\theta}^{(k)}=\left(\boldsymbol{\beta}^{(k)^{\top}}, \sigma^{2(k)}, \lambda^{(k)}\right)^{\top}$ a estimativa de $\boldsymbol{\theta}$ na $k$-ésima iteração. Segue que a esperança com respeito a $\mathbf{t}$ e $\mathbf{u}$, condicionada em $\mathbf{y}$, da função log-verossimilhança completa (Passo E), tem a forma

$$
\begin{aligned}
Q\left(\boldsymbol{\theta} \mid \widehat{\boldsymbol{\theta}}^{(k)}\right)= & \mathrm{E}\left[\ell_{c}\left(\boldsymbol{\theta} \mid \mathbf{y}_{c}\right) \mid \mathbf{y}, \widehat{\boldsymbol{\theta}}^{(k)}\right] \\
= & -n \log {\sigma^{2}}^{(k)}-\frac{1}{2 \sigma^{2(k)}} \sum_{i=1}^{n}{\widehat{t^{2}}}_{i}^{(k)}+\frac{\lambda^{(k)}}{\sigma^{2^{(k)}}} \sum_{i=1}^{n} \widehat{t}_{i}^{(k)}\left(y_{i}-\mathbf{x}_{i}^{\top} \boldsymbol{\beta}^{(k)}\right) \\
& -\frac{1}{2 \sigma^{2(k)}} \sum_{i=1}^{n}\left(\widehat{\kappa}_{i}^{(k)}+\lambda^{(k)^{2}}\right)\left(y_{i}-\mathbf{x}_{i}^{\top} \boldsymbol{\beta}^{(k)}\right)^{2} .
\end{aligned}
$$

Assim, tem-se o seguinte algoritmo EM:

Passo E: Dado $\boldsymbol{\theta}=\widehat{\boldsymbol{\theta}}^{(k)}$, calcule para $i=1, \ldots, n, \widehat{t}_{i}^{(k)},{\widehat{t^{2}}}^{(k)}$ usando (4.23) e $\widehat{\kappa}_{i}^{(k)}$ usando (4.26)-(4.29).

Passo M: Atualize $\widehat{\boldsymbol{\theta}}^{(k+1)}$ maximizando $Q\left(\boldsymbol{\theta} \mid \widehat{\boldsymbol{\theta}}^{(k)}\right)$ em relação a $\boldsymbol{\theta}$, o qual leva às seguintes 
expressões:

$$
\begin{aligned}
& \widehat{\boldsymbol{\beta}}^{(k+1)}=\left[\mathbf{X}^{\top}\left(\mathbf{D}\left(\widehat{\boldsymbol{\kappa}}^{(k)}\right)+\lambda^{(k)^{2}} \mathbb{I}_{n}\right) \mathbf{X}\right]^{-1} \mathbf{X}^{\top}\left[\mathbf{D}\left(\widehat{\boldsymbol{\kappa}}^{(k)}\right) \mathbf{y}-\lambda^{(k)}\left(\widehat{\mathbf{t}}^{(k)}-\lambda^{(k)} \mathbf{y}\right)\right], \\
& {\widehat{\sigma^{2}}}^{(k+1)}=\frac{1}{2 n}\left[Q_{\widehat{\boldsymbol{\kappa}}^{(k)}}\left(\boldsymbol{\beta}^{(k)}\right)+\widehat{\mathbf{t}}^{(k)^{\top}} \mathbf{1}_{n}-2 \lambda^{(k)} \widehat{\mathbf{t}}^{(k)^{\top}}\left(\mathbf{y}-\mathbf{X} \boldsymbol{\beta}^{(k)}\right)+\lambda^{(k)^{2}} Q\left(\boldsymbol{\beta}^{(k)}\right)\right] \mathrm{e} \\
& \widehat{\lambda}^{(k+1)}=\frac{\widehat{\mathbf{t}}^{(k)^{\top}}\left(\mathbf{y}-\mathbf{X} \boldsymbol{\beta}^{(k)}\right)}{Q\left(\boldsymbol{\beta}^{(k)}\right)},
\end{aligned}
$$

onde $Q\left(\boldsymbol{\beta}^{(k)}\right)=\left(\mathbf{y}-\mathbf{X} \boldsymbol{\beta}^{(k)}\right)^{\top}\left(\mathbf{y}-\mathbf{X} \boldsymbol{\beta}^{(k)}\right), Q_{\widehat{\boldsymbol{\kappa}}^{(k)}}\left(\boldsymbol{\beta}^{(k)}\right)=\left(\mathbf{y}-\mathbf{X} \boldsymbol{\beta}^{(k)}\right)^{\top} \mathbf{D}\left(\widehat{\boldsymbol{\kappa}}^{(k)}\right)\left(\mathbf{y}-\mathbf{X} \boldsymbol{\beta}^{(k)}\right)$, $\mathbf{1}_{n}(n \times 1)$ é um vetor de 1 's, $\mathbb{I}_{n}$ é a matriz identidade de ordem $n, \mathbf{X}^{\top}=\left(\mathbf{x}_{1} \ldots, \mathbf{x}_{n}\right)$ é uma matriz de ordem $\left.p \times n, \widehat{\boldsymbol{\kappa}}^{(k)}=\left[\widehat{\kappa}_{1}^{(k)}, \ldots, \widehat{\kappa}_{n}^{(k)}\right]^{\top}, \mathbf{D}\left(\boldsymbol{\kappa}^{(k)}\right)=\operatorname{Diag}\left(\widehat{\kappa}_{1}^{(k)}, \ldots, \widehat{\kappa}_{n}^{(k)}\right)\right)$.

\subsubsection{Distribuições condicionais para o algoritmo EM}

Nesta seção são calculadas as esperanças condicionais $E[U \mid y]$ para as distribuições presentes na seção 3, as quais são usadas no algoritmo EM. Antes, segue um importante resultado.

Proposição 4.6. Se $Y \sim M E N A(\boldsymbol{\theta} ; H), \boldsymbol{\theta}=\left(\mu, \sigma^{2}, \lambda\right)^{\top}$, então a distribuição condicional de $U \mid Y=y$ não depende de $\lambda$.

Demonstração.

$$
\begin{aligned}
f(u \mid y, \boldsymbol{\theta}) & =\frac{f(u, y \mid \boldsymbol{\theta})}{f(y \mid \boldsymbol{\theta})} \\
& =\frac{2 \phi\left(y \mid \mu, \sigma^{2} \kappa(u)\right) \Phi\left(\frac{\lambda(y-\mu)}{\sigma}\right) h(u ; \boldsymbol{\tau})}{2 \int_{0}^{\infty} \phi\left(y \mid \mu, \sigma^{2} \kappa(u)\right) \Phi\left(\frac{\lambda(y-\mu)}{\sigma}\right) h(u ; \boldsymbol{\tau}) d u} \\
& =\frac{\phi\left(y \mid \mu, \sigma^{2} \kappa(u)\right) h(u ; \boldsymbol{\tau})}{\int_{0}^{\infty} \phi\left(y \mid \mu, \sigma^{2} \kappa(u)\right) h(u ; \boldsymbol{\tau}) d u} .
\end{aligned}
$$

Da Proposição 4.6 segue que, sob a distribuição $M E N A$ mais geral considerada aqui, a distribuição condicional $U_{i} \mid Y_{i}$ reduz à distribuição $M E N$. Essa peculiaridade simplifica bastante a implementação do algoritmo EM. Assim, para as distribuições discutidas, dado $\boldsymbol{\theta}=\left(\mu, \sigma^{2}, \lambda\right)$, tem-se os seguintes resultados: 
- Distribuição t de Student generalizada normal assimétrica. Se $Y \sim G t A\left(\mu, \sigma^{2}, \lambda ; \nu, \gamma\right)$, então, de (4.4) juntamente com a Proposição 4.6, tem-se que

$$
\begin{aligned}
& f(u \mid y, \boldsymbol{\theta})=\frac{(\gamma / 2)^{\nu / 2}}{\Gamma(\nu / 2)} u^{\nu / 2-1} e^{-\gamma u / 2} \frac{u^{1 / 2}}{\sqrt{2 \pi} \sigma} e^{-u d / 2} \\
& U \mid Y=y \sim \operatorname{Gama}\left(\frac{\nu+1}{2}, \frac{\gamma+d}{2}\right)
\end{aligned}
$$

$\operatorname{com} d=(y-\mu)^{2} / \sigma^{2}$. Assim, $E_{U}\left[U^{\alpha} \mid y, \boldsymbol{\theta}\right]=\frac{\Gamma\left(\frac{\nu+1}{2}+\alpha\right)}{\Gamma\left(\frac{\nu+1}{2}\right)\left(\frac{\gamma+d}{2}\right)^{\alpha}}, \alpha>0$ e então

$$
\widehat{\kappa}=\frac{\nu+1}{\gamma+d} \text {. }
$$

- Distribuição slash assimétrica. Se $Y \sim S L A\left(\mu, \sigma^{2}, \lambda ; \nu\right)$, obtém-se

$$
\begin{aligned}
& f(u \mid y, \boldsymbol{\theta})=\nu u^{\nu-1} \mathbf{I}_{(0,1)}(u) \frac{u^{1 / 2}}{\sqrt{2 \pi} \sigma} e^{-u d / 2} \\
& U \mid Y=y \sim \operatorname{Gama}(\nu+1 / 2, d / 2) \mathbf{I}_{(0,1)}(u) .
\end{aligned}
$$

Assim, $E_{U}\left(U^{\alpha} \mid y, \boldsymbol{\theta}\right)=\frac{\Gamma(\nu+1 / 2+\alpha)}{\Gamma(\nu+1 / 2)(d / 2)^{\alpha}} \frac{P_{1}(\nu+\alpha+1 / 2, d / 2)}{P_{1}(\nu+1 / 2, d / 2)}, \alpha>0 \mathrm{e}$

$$
\widehat{\kappa}=\frac{(2 \nu+1)}{d} \frac{P_{1}(\nu+3 / 2, d / 2)}{P_{1}(\nu+1 / 2, d / 2)}
$$

onde $P_{x}(a, b)$ denota a fda de uma distribuição $\operatorname{Gama}(a, b)$ avaliada em $x$.

- Distribuição normal contaminada assimétrica. Se $Y \sim N C A\left(\mu, \sigma^{2}, \lambda ; \nu, \gamma\right)$, tem-se que

$$
\begin{gathered}
f(u \mid y, \boldsymbol{\theta})=\nu p \mathbf{I}_{(u=\gamma)}+(1-\nu) p \mathbf{I}_{(u=1)}, \\
\operatorname{com} p=\frac{u^{1 / 2} \exp \left(-\frac{d u}{2}\right)}{\nu \gamma^{1 / 2} \exp \left(-\frac{d \gamma}{2}\right)+(1-\nu) \exp \left(-\frac{d}{2}\right)} . \\
\text { Mais ainda, } E_{U}\left(U^{\alpha} \mid y, \boldsymbol{\theta}\right)=\frac{1-\nu+\nu \gamma^{\alpha+1 / 2} \exp \{(1-\gamma) d / 2\}}{1-\nu+\nu \gamma^{1 / 2} \exp \{(1-\gamma) d / 2\}}, \alpha>0 \mathrm{e} \\
\widehat{\kappa}=\frac{1-\nu+\nu \gamma^{3 / 2} \exp \{(1-\gamma) d / 2\}}{1-\nu+\nu \gamma^{1 / 2} \exp \{(1-\gamma) d / 2\}} .
\end{gathered}
$$


- Distribuição exponencial potência assimétrica. Nesse caso temos a seguinte proposição:

Proposição 4.7. Se $Y \sim E P A\left(\mu, \sigma^{2}, \lambda ; \nu\right)$, então

$$
\widehat{\kappa}=E_{U}\left[\kappa^{-1}(U) \mid y, \boldsymbol{\theta}\right]=\nu d^{\nu-1}
$$

Demonstração. Sendo $Y \sim M E N\left(\mu, \sigma^{2} ; \nu\right)$, tem-se que

$$
f_{Y}(y)=\int_{0}^{+\infty} \phi\left(y \mid \mu, \sigma^{2} \kappa(u)\right) h(u ; \nu) d u
$$

e $f_{(U, Y)}(u, y)=\phi\left(y \mid \mu, \sigma^{2} \kappa(u)\right) h(u ; \nu)$. Como $V=\kappa^{-1}(U), \kappa(U)=1 / V$, então

$$
f_{(V, Y)}(v, y)=f_{(U, Y)}(u, y)|J| \propto \phi\left(y \mid \mu, \sigma^{2} / v\right) h\left(\kappa^{-1}(1 / v) ; \nu\right)
$$

onde $J$ é o Jacobiano da transformação $g(u, y)=\left(\kappa^{-1}(u), y\right)$. Então,

$$
\begin{aligned}
f_{(V \mid Y=y)}(v \mid y) & =\frac{\phi\left(y \mid \mu, \sigma^{2} / v\right) h\left(k^{-1}(1 / v) ; \nu\right)}{f_{Y}(y)} \\
& =h\left(\kappa^{-1}(1 / v) ; \nu\right) \frac{2^{\frac{1}{2 \nu}} \sigma \Gamma\left(\frac{1}{2 \nu}\right)}{\nu} e^{d^{\nu} / 2} \frac{v^{1 / 2}}{\sqrt{2 \pi} \sigma} e^{-\frac{(y-u)^{2}}{2 \sigma^{2}} v} \\
& \propto \exp \left\{\frac{1}{2}\left[-d v+d^{\nu}\right]+c(v, \nu)\right\} .
\end{aligned}
$$

Assim, $\kappa^{-1}(U) \mid Y$ pertence à família exponencial, com parâmetro $\theta=-d, b(\theta)=-d^{\nu}=$ $-(-\theta)^{\nu}$ e parâmetro de dispersão $\phi^{-1}=2$. Assim, $\widehat{\kappa}=E_{U}\left[\kappa^{-1}(U) \mid y, \boldsymbol{\theta}\right]=b^{\prime}(\theta)=\nu d^{\nu-1}$.

\subsection{Matriz de Informação de Fisher Observada}

Considere $Y_{1}, \ldots, Y_{n}$, onde $Y_{i} \sim \operatorname{MENA}\left(\mu_{i}, \sigma^{2}, \lambda ; H\right), \mu_{i}=\mathbf{x}_{i}^{\top} \boldsymbol{\beta}, i=1, \ldots, n$, onde $\boldsymbol{\beta}$ é um vetor $p$-dimensional de coeficientes de regressão desconhecidos. Então a função log-verossimilhança $\ell(\boldsymbol{\theta})=\sum_{i=1}^{n} \ell_{i}(\boldsymbol{\theta})$ é da forma

$$
\ell_{i}(\boldsymbol{\theta})=\log 2+\ell_{1_{i}}(\boldsymbol{\theta})+\log \left[\Phi\left(\ell_{2_{i}}(\boldsymbol{\theta})\right)\right], \quad \boldsymbol{\theta}=\left(\boldsymbol{\beta}^{\top}, \sigma^{2}, \lambda\right)^{\top}
$$

Ferreira, C. S. 
onde $\ell_{1}(\boldsymbol{\theta})$ é a função de log-verossimilhança da distribuição simétrica $M E N$ e $\ell_{2_{i}}(\boldsymbol{\theta})=$ $\lambda \frac{y_{i}-\mathbf{x}_{i}^{\top} \boldsymbol{\beta}}{\sigma}$. Então, a primeira derivada de $\ell_{i}(\boldsymbol{\theta})$ é dada por

$$
\frac{\partial \ell_{i}(\boldsymbol{\theta})}{\partial \boldsymbol{\psi}}=\frac{\partial \ell_{1_{i}}(\boldsymbol{\theta})}{\partial \boldsymbol{\psi}}+W_{\Phi}\left(l_{2_{i}}(\boldsymbol{\theta})\right) \frac{\partial \ell_{2_{i}}(\boldsymbol{\theta})}{\partial \boldsymbol{\psi}}, \quad \boldsymbol{\psi}=\boldsymbol{\beta}, \sigma^{2}, \lambda
$$

onde $W_{\Phi}(x)=\frac{\phi(x)}{\Phi(x)}$. A segunda derivada é dada por

$$
\frac{\partial^{2} \ell_{i}(\boldsymbol{\theta})}{\partial \boldsymbol{\gamma} \partial \boldsymbol{\psi}^{\top}}=\frac{\partial^{2} \ell_{1_{i}}(\boldsymbol{\theta})}{\partial \boldsymbol{\gamma} \partial \boldsymbol{\psi}^{\top}}+W_{\Phi}\left(\ell_{2_{i}}(\boldsymbol{\theta})\right) \frac{\partial^{2} \ell_{2_{i}}(\boldsymbol{\theta})}{\partial \boldsymbol{\gamma} \partial \boldsymbol{\psi}^{\top}}+W_{\Phi}^{(1)}\left(\ell_{2_{i}}(\boldsymbol{\theta})\right) \frac{\partial \ell_{2_{i}}(\boldsymbol{\theta})}{\partial \boldsymbol{\gamma}} \frac{\partial \ell_{2_{i}}(\boldsymbol{\theta})}{\partial \boldsymbol{\psi}^{\top}}
$$

onde $W_{\Phi}^{(1)}(x)=-W_{\Phi}(x)\left(x+W_{\Phi}(x)\right)$ é a derivada de $W_{\Phi}(x)$.

Portanto, a matriz de informação observada para $\boldsymbol{\theta}$ pode ser escrita como

$$
\begin{gathered}
\mathbf{I}(\boldsymbol{\theta})=I_{1}(\boldsymbol{\theta})+I_{2}(\boldsymbol{\theta}) \\
\operatorname{com} I_{k}(\boldsymbol{\theta})=\left(\begin{array}{ccc}
I_{\boldsymbol{\beta} \boldsymbol{\beta}}^{k} & I_{\sigma^{2} \boldsymbol{\beta}}^{k} & I_{\lambda \boldsymbol{\beta}}^{k} \\
& I_{\sigma^{2} \sigma^{2}}^{k} & I_{\lambda \sigma^{2}}^{k} \\
& & I_{\lambda \lambda}^{k}
\end{array}\right),
\end{gathered}
$$

para $k=1,2, \boldsymbol{\gamma}, \boldsymbol{\psi}=\boldsymbol{\beta}, \sigma^{2}, \lambda, \mathrm{e}$

$$
\begin{aligned}
I_{\boldsymbol{\gamma} \boldsymbol{\psi}^{\top}}^{1} & =-\sum_{i=1}^{n} \frac{\partial^{2} \ell_{1_{i}}(\boldsymbol{\theta})}{\partial \boldsymbol{\gamma} \partial \boldsymbol{\psi}^{\top}}, \\
I_{\boldsymbol{\gamma} \boldsymbol{\psi}^{\top}}^{2} & =-\sum_{i=1}^{n}\left[W_{\Phi}\left(\ell_{2_{i}}(\boldsymbol{\theta})\right) \frac{\partial^{2} \ell_{2_{i}}(\boldsymbol{\theta})}{\partial \boldsymbol{\gamma} \partial \boldsymbol{\psi}^{\top}}+W_{\Phi}^{(1)}\left(\ell_{2_{i}}(\boldsymbol{\theta})\right) \frac{\partial \ell_{2_{i}}(\boldsymbol{\theta})}{\partial \boldsymbol{\gamma}} \frac{\partial \ell_{2_{i}}(\boldsymbol{\theta})}{\partial \boldsymbol{\psi}^{\top}}\right] .
\end{aligned}
$$

Ferreira, C. S. 
Para todas distribuições na classe MEN, os elementos de $I_{2}(\boldsymbol{\theta})$ são comuns e são dados por

$$
\begin{aligned}
I_{\boldsymbol{\beta} \boldsymbol{\beta}}^{2} & =-\frac{\lambda^{2}}{\sigma^{2}} \mathbf{X}^{\top} \mathbf{D}\left(W_{\Phi}^{(1)}\left[\lambda \frac{(\mathbf{y}-\mathbf{X} \boldsymbol{\beta})}{\sigma}\right]\right) \mathbf{X} \\
I_{\sigma^{2} \boldsymbol{\beta}}^{2} & =-\frac{\lambda}{2 \sigma^{3}} \mathbf{X}^{\top} W_{\Phi}\left[\lambda \frac{(\mathbf{y}-\mathbf{X} \boldsymbol{\beta})}{\sigma}\right]-\frac{\lambda^{2}}{2 \sigma^{4}} \mathbf{X}^{\top} \mathbf{D}\left(W_{\Phi}^{(1)}\left[\lambda \frac{(\mathbf{y}-\mathbf{X} \boldsymbol{\beta})}{\sigma}\right]\right)(\mathbf{y}-\mathbf{X} \boldsymbol{\beta}), \\
I_{\lambda \boldsymbol{\beta}}^{2} & =\frac{1}{\sigma} \mathbf{X}^{\top} W_{\Phi}\left[\lambda \frac{(\mathbf{y}-\mathbf{X} \boldsymbol{\beta})}{\sigma}\right]+\frac{\lambda}{\sigma^{2}} \mathbf{X}^{\top} \mathbf{D}\left(W_{\Phi}^{(1)}\left[\lambda \frac{(\mathbf{y}-\mathbf{X} \boldsymbol{\beta})}{\sigma}\right]\right)(\mathbf{y}-\mathbf{X} \boldsymbol{\beta}), \\
I_{\sigma^{2} \sigma^{2}}^{2} & =-\frac{3 \lambda}{4 \sigma^{5}}(\mathbf{y}-\mathbf{X} \boldsymbol{\beta})^{\top} W_{\Phi}\left[\lambda \frac{(\mathbf{y}-\mathbf{X} \boldsymbol{\beta})}{\sigma}\right]-\frac{\lambda^{2}}{4 \sigma^{6}} Q_{w}(\boldsymbol{\beta}), \\
I_{\lambda \sigma^{2}}^{2} & =\frac{1}{2 \sigma^{3}}(\mathbf{y}-\mathbf{X} \boldsymbol{\beta})^{\top} W_{\Phi}\left[\lambda \frac{(\mathbf{y}-\mathbf{X} \boldsymbol{\beta})}{\sigma}\right]+\frac{\lambda}{2 \sigma^{4}} Q_{w}(\boldsymbol{\beta}) \mathrm{e} \\
I_{\lambda \lambda}^{2} & =-\frac{1}{\sigma^{2}} Q_{w}(\boldsymbol{\beta}),
\end{aligned}
$$

onde $Q_{w}(\boldsymbol{\beta})=(\mathbf{y}-\mathbf{X} \boldsymbol{\beta})^{\top} \mathbf{D}\left(W_{\Phi}^{(1)}\left[\lambda \frac{(\mathbf{y}-\mathbf{X} \boldsymbol{\beta})}{\sigma}\right]\right)(\mathbf{y}-\mathbf{X} \boldsymbol{\beta}), \mathbf{D}(\mathbf{a})$ é a matriz diagonal do vetor a. Note que sendo $W_{\Phi}(x)=\phi(x) / \Phi(x)$, com $\phi($.$) e \Phi($.$) as funções densidade e acumulada$ da distribuição normal padrão, então $-1 \leq W_{\Phi}^{(1)}(x) \leq 0$.

A matriz $I_{1}(\boldsymbol{\theta})$ pode ser calculada para cada distribuição MEN considerada, como segue:

- Distribuição t de Student generalizada, $Y \sim G t\left(\mu, \sigma^{2} ; \nu, \gamma\right)$.

$$
\begin{aligned}
I_{\boldsymbol{\beta} \boldsymbol{\beta}}^{1} & =-\frac{\nu+1}{\gamma \sigma^{2}} \mathbf{X}^{\top}\left(\frac{2}{\gamma \sigma^{2}} \mathbf{D}^{2}(\mathbf{V}) \mathbf{D}^{2}(\mathbf{y}-\mathbf{X} \boldsymbol{\beta})-\mathbf{D}(\mathbf{V})\right) \mathbf{X} \\
I_{\sigma^{2} \boldsymbol{\beta}}^{1} & =-\frac{\nu+1}{\gamma \sigma^{4}} \mathbf{X}^{\top} \mathbf{D}(\mathbf{y}-\mathbf{X} \boldsymbol{\beta})\left(\frac{1}{\gamma \sigma^{2}} \mathbf{D}^{2}(\mathbf{V}) \mathbf{D}(\mathbf{y}-\mathbf{X} \boldsymbol{\beta})(\mathbf{y}-\mathbf{X} \boldsymbol{\beta})-\mathbf{V}\right), \\
I_{\sigma^{2} \sigma^{2}}^{1} & =-\frac{n}{2 \sigma^{4}}+\frac{\nu+1}{\gamma \sigma^{6}} Q_{\mathbf{V}}(\boldsymbol{\beta})-\frac{\nu+1}{2 \gamma^{2} \sigma^{8}}(\mathbf{y}-\mathbf{X} \boldsymbol{\beta})^{\top} \mathbf{D}^{3}(\mathbf{y}-\mathbf{X} \boldsymbol{\beta}) \mathbf{D}(\mathbf{V}) \mathbf{V} \mathrm{e} \\
I_{\lambda \boldsymbol{\tau}}^{1} & =\mathbf{0}, \boldsymbol{\tau}=\boldsymbol{\beta}, \sigma^{2}, \lambda
\end{aligned}
$$

onde $Q_{\mathbf{V}}(\boldsymbol{\beta})=(\mathbf{y}-\mathbf{X} \boldsymbol{\beta})^{\top} \mathbf{D}(\mathbf{V})(\mathbf{y}-\mathbf{X} \boldsymbol{\beta})$ and $\mathbf{V}=\left(V_{1}, \ldots, V_{n}\right)^{\top}, V_{i}=\left(1+\frac{d_{i}}{\gamma}\right)^{-1}$, $d_{i}=\frac{\left(y_{i}-\mathbf{x}_{i}^{\top} \boldsymbol{\beta}\right)^{2}}{\sigma^{2}}, i=1, \ldots, n$. Como pode ser percebido em Gómez et al. (2007), a matriz de informação do modelo t-normal assimétrico é não-singular para um número graus de liberdade finito quando $\lambda=0$. Por outro lado, as distribuições normal assimétrica e t-assimétrica são singulares em $\lambda=0$ (Pewsey, 2000).

Ferreira, C. S. 
- Distribuição slash, $Y \sim S L\left(\mu, \sigma^{2} ; \nu\right)$.

$$
\begin{aligned}
I_{\boldsymbol{\beta} \boldsymbol{\beta}}^{1}= & -(2 \nu+1) \mathbf{X}^{\top} \mathbf{D}\left[(\mathbf{y}-\mathbf{X} \boldsymbol{\beta})^{-2}\right] \mathbf{D}\left[P_{1}(\nu+1 / 2, \mathbf{d} / 2)^{-1}\right] \\
& \times\left[(2 \nu+3) \mathbf{D}\left[P_{1}(\nu+5 / 2, \mathbf{d} / 2)\right]-\mathbf{D}\left[P_{1}(\nu+3 / 2, \mathbf{d} / 2)\right]\right. \\
& \left.-(2 \nu+1) \mathbf{D}\left[P_{1}(\nu+3 / 2, \mathbf{d} / 2)^{2}\right] \mathbf{D}\left[P_{1}(\nu+1 / 2, \mathbf{d} / 2)^{-1}\right]\right] \mathbf{X}, \\
I_{\sigma^{2} \boldsymbol{\beta}}^{1}= & -\frac{(2 \nu+1)}{2 \sigma^{2}} \mathbf{X}^{\top} \mathbf{D}\left[(\mathbf{y}-\mathbf{X} \boldsymbol{\beta})^{-1}\right] \mathbf{D}\left[P_{1}(\nu+1 / 2, \mathbf{d} / 2)^{-1}\right] \\
& \times\left[(2 \nu+3) P_{1}(\nu+5 / 2, \mathbf{d} / 2)-2 P_{1}(\nu+3 / 2, \mathbf{d} / 2)\right. \\
& \left.-(2 \nu+1) \mathbf{D}\left[P_{1}(\nu+3 / 2, \mathbf{d} / 2)^{2}\right] P_{1}(\nu+1 / 2, \mathbf{d} / 2)^{-1}\right], \\
I_{\sigma^{2} \sigma^{2}}^{1}= & -\frac{n}{2 \sigma^{4}}-\frac{(2 \nu+1)}{4 \sigma^{4}}\left(P_{1}(\nu+1 / 2, \mathbf{d} / 2)^{-1}\right)^{\top} \\
& \times\left[(2 \nu+3) P_{1}(\nu+5 / 2, \mathbf{d} / 2)-4 P_{1}(\nu+3 / 2, \mathbf{d} / 2)\right. \\
& \left.-(2 \nu+1) \mathbf{D}\left[\left(P_{1}(\nu+3 / 2, \mathbf{d} / 2)^{2}\right)\right] P_{1}(\nu+1 / 2, \mathbf{d} / 2)^{-1}\right] \mathrm{e} \\
I_{\lambda \boldsymbol{\tau}}^{1}= & \mathbf{0}, \boldsymbol{\tau}=\boldsymbol{\beta}, \sigma^{2}, \lambda,
\end{aligned}
$$

onde $\mathbf{d}=\left(d_{1}, \ldots, d_{n}\right)^{\top}, d_{i}=\left(y_{i}-\mathbf{x}_{i}^{\top}\right)^{2} / \sigma^{2}$

- Distribuição normal contaminada, $Y \sim N C\left(\mu, \sigma^{2} ; \nu, \gamma\right)$.

$$
\begin{aligned}
I_{\boldsymbol{\beta} \beta}^{1} & =\frac{\gamma+1}{\sigma^{2}} \mathbf{X}^{\top} \mathbf{X}, \\
I_{\sigma^{2} \boldsymbol{\beta}}^{1} & =\frac{\gamma+1}{\sigma^{4}} \mathbf{X}^{\top}(\mathbf{y}-\mathbf{X} \boldsymbol{\beta}), \\
I_{\sigma^{2} \sigma^{2}}^{1} & =-\frac{n}{\sigma^{4}}+\frac{\gamma+1}{\sigma^{6}}(\mathbf{y}-\mathbf{X} \boldsymbol{\beta})^{\top}(\mathbf{y}-\mathbf{X} \boldsymbol{\beta}) \mathrm{e} \\
I_{\lambda \boldsymbol{\tau}}^{1} & =\mathbf{0}, \boldsymbol{\tau}=\boldsymbol{\beta}, \sigma^{2}, \lambda .
\end{aligned}
$$

- Distribuição exponencial potência, $Y \sim E P\left(\mu, \sigma^{2} ; \nu\right)$.

$$
\begin{aligned}
I_{\boldsymbol{\beta} \boldsymbol{\beta}}^{1} & =\frac{\nu(2 \nu-1)}{\sigma^{2 \nu}} \mathbf{X}^{\top} \mathbf{D}\left[(\mathbf{y}-\mathbf{X} \boldsymbol{\beta})^{2 \nu-2}\right] \mathbf{X}, \\
I_{\sigma^{2} \boldsymbol{\beta}}^{1} & =\frac{\nu^{2}}{\sigma^{2(\nu+1)}} \mathbf{X}^{\top}\left[(\mathbf{y}-\mathbf{X} \boldsymbol{\beta})^{2 \nu-1}\right], \\
I_{\sigma^{2} \sigma^{2}}^{1} & =-\frac{n}{2 \sigma^{4}}+\frac{\nu(\nu+1)}{2 \sigma^{2(\nu+2)}}\left[(\mathbf{y}-\mathbf{X} \boldsymbol{\beta})^{\nu}\right]^{\top}\left[(\mathbf{y}-\mathbf{X} \boldsymbol{\beta})^{\nu}\right] \mathrm{e} \\
I_{\lambda \boldsymbol{\tau}}^{1} & =\mathbf{0}, \boldsymbol{\tau}=\boldsymbol{\beta}, \sigma^{2}, \lambda .
\end{aligned}
$$

Intervalos de confiança assintóticos e testes de hipóteses (por exemplo, teste da razão de verossimilhança) para os parâmetros do modelo de regressão MENA podem ser obtidos 
usando a matriz $\mathbf{I}(\boldsymbol{\theta})$. Na prática, $\mathbf{I}(\boldsymbol{\theta})$ é substituída pelo EMV $\mathbf{I}(\widehat{\boldsymbol{\theta}})$.

\subsection{Estudo de Convergência do Algoritmo EM}

Para demonstrar a robustez do algoritmo EM no sentido de estimação para os modelos de mistura de escala normais assimétricas, um estudo de simulação foi conduzido. Para tanto, para cada distribuição considerada (normal, t-normal, Slash, normal contaminada e exponencial potência), foi considerado um modelo de regressão simples

$$
y_{i}=\alpha+\beta x_{i}+\epsilon_{i}
$$

com $x_{i} \sim U(0,1), \epsilon_{i} \sim \operatorname{MENA}\left(0, \sigma^{2}, \lambda ; H\right)$, com tamanho de amostra $n=300$ e número de replicações $M=1000$. Os parâmetros considerados foram $\boldsymbol{\theta}^{\top}=\left(\alpha, \beta, \sigma^{2}, \lambda\right)=(1,2,0.5,3)$. Os parâmetros $\tau$ para cada distribuição foram $\nu=\gamma=5$ para t-normal, $\nu=0.1, \gamma=0.95$ para normal contaminada, $\nu=5$ para slash e $\nu=0.8$ para exponencial potência. As tabelas 4.1-4.5 apresentam a média dos estimadores de $\boldsymbol{\theta}$ nas $M$ replicações, juntamente com a média dos erros padrão empíricos (EP) obtidos através da matriz de informação observada (MI) (Seção 4.5) em cada uma das $M$ amostras replicadas e também o desvio padrão amostral das $M$ estimativas obtidas dos parâmetros.

Tabela 4.1: Modelo normal assimétrico: média das $M$ estimativas $\left(\overline{\boldsymbol{\theta}}=\sum_{k=1}^{M} \widehat{\boldsymbol{\theta}}_{k} / M\right)$, erro padrão assintótico obtido através da MI $\left(E P(\widehat{\boldsymbol{\theta}})=\left[\operatorname{Diag}\left(\mathbf{I}_{-1}(\widehat{\boldsymbol{\theta}})\right)\right]^{1 / 2}\right)$ e erro padrão empírico das $M$ estimativas $\left(E P_{e m p}=\left[\sum_{k=1}^{M}\left(\widehat{\boldsymbol{\theta}}_{k}-\overline{\widehat{\boldsymbol{\theta}}}\right)^{2} / M\right]^{1 / 2}\right)$.

\begin{tabular}{c|c|cc}
\hline \hline & $\overline{\widehat{\boldsymbol{\theta}}}$ & $E P(\widehat{\boldsymbol{\theta}})$ & $E P_{e m p}$ \\
\hline$\alpha$ & 1.003 & 0.058 & 0.059 \\
$\beta$ & 1.999 & 0.079 & 0.082 \\
$\sigma^{2}$ & 0.497 & 0.061 & 0.062 \\
$\lambda$ & 3.140 & 0.674 & 0.702 \\
\hline \hline
\end{tabular}

Ferreira, C. S. 
Tabela 4.2: Modelo $\mathrm{t}$ de Student generalizado normal assimétrico: média das $M$ estimativas $\left(\overline{\boldsymbol{\theta}}=\sum_{k=1}^{M} \widehat{\boldsymbol{\theta}}_{k} / M\right)$, erro padrão assintótico obtido através da MI $\left(E P(\widehat{\boldsymbol{\theta}})=\left[\operatorname{Diag}\left(\mathbf{I}_{-1}(\widehat{\boldsymbol{\theta}})\right)\right]^{1 / 2}\right)$ e erro padrão empírico das $M$ estimativas $\left(E P_{e m p}=\right.$ $\left.\left[\sum_{k=1}^{M}\left(\widehat{\boldsymbol{\theta}}_{k}-\overline{\widehat{\boldsymbol{\theta}}}\right)^{2} / M\right]^{1 / 2}\right)$.

\begin{tabular}{c|c|cc}
\hline \hline & $\overline{\widehat{\boldsymbol{\theta}}}$ & $E P(\widehat{\boldsymbol{\theta}})$ & $E P_{e m p}$ \\
\hline$\alpha$ & 1.002 & 0.061 & 0.064 \\
$\beta$ & 2.001 & 0.085 & 0.086 \\
$\sigma^{2}$ & 0.508 & 0.075 & 0.075 \\
$\lambda$ & 3.161 & 0.739 & 0.782 \\
\hline \hline
\end{tabular}

Tabela 4.3: Modelo slash assimétrico: média das $M$ estimativas $\left(\overline{\widehat{\boldsymbol{\theta}}}=\sum_{k=1}^{M} \widehat{\boldsymbol{\theta}}_{k} / M\right)$, erro padrão assintótico obtido através da MI $\left(E P(\widehat{\boldsymbol{\theta}})=\left[\operatorname{Diag}\left(\mathbf{I}_{-1}(\widehat{\boldsymbol{\theta}})\right)\right]^{1 / 2}\right)$ e erro padrão empírico das $M$ estimativas $\left(E P_{e m p}=\left[\sum_{k=1}^{M}\left(\widehat{\boldsymbol{\theta}}_{k}-\overline{\widehat{\boldsymbol{\theta}}}\right)^{2} / M\right]^{1 / 2}\right)$.

\begin{tabular}{c|c|cc}
\hline \hline & $\overline{\widehat{\boldsymbol{\theta}}}$ & $E P(\widehat{\boldsymbol{\theta}})$ & $E P_{e m p}$ \\
\hline$\alpha$ & 1.000 & 0.061 & 0.062 \\
$\beta$ & 2.005 & 0.081 & 0.082 \\
$\sigma^{2}$ & 0.496 & 0.061 & 0.060 \\
$\lambda$ & 3.114 & 0.676 & 0.689 \\
\hline \hline
\end{tabular}

Tabela 4.4: Modelo normal contaminado assimétrico: média das $M$ estimativas $(\overline{\boldsymbol{\theta}}=$ $\left.\sum_{k=1}^{M} \widehat{\boldsymbol{\theta}}_{k} / M\right)$, erro padrão assintótico obtido através da MI $\left(E P(\widehat{\boldsymbol{\theta}})=\left[\operatorname{Diag}\left(\mathbf{I}_{-1}(\widehat{\boldsymbol{\theta}})\right)\right]^{1 / 2}\right)$ e erro padrão empírico das $M$ estimativas $\left(E P_{e m p}=\left[\sum_{k=1}^{M}\left(\widehat{\boldsymbol{\theta}}_{k}-\widehat{\widehat{\boldsymbol{\theta}}}\right)^{2} / M\right]^{1 / 2}\right)$.

\begin{tabular}{c|c|cc}
\hline \hline & $\overline{\widehat{\boldsymbol{\theta}}}$ & $E P(\widehat{\boldsymbol{\theta}})$ & $E P_{e m p}$ \\
\hline$\alpha$ & 1.005 & 0.061 & 0.064 \\
$\beta$ & 1.998 & 0.073 & 0.085 \\
$\sigma^{2}$ & 0.497 & 0.060 & 0.062 \\
$\lambda$ & 3.119 & 0.726 & 0.693 \\
\hline \hline
\end{tabular}

De acordo com as Tabelas 4.1-4.5, percebe-se que o algoritmo EM se comporta bem em todos os casos, sendo que no modelo exponencial potência assimétrico, as estimativas dos 
Tabela 4.5: Modelo exponencial potência assimétrico: média das $M$ estimativas $(\overline{\boldsymbol{\theta}}=$ $\left.\sum_{k=1}^{M} \widehat{\boldsymbol{\theta}}_{k} / M\right)$, erro padrão assintótico obtido através da MI $\left(\operatorname{EP}(\widehat{\boldsymbol{\theta}})=\left[\operatorname{Diag}\left(\mathbf{I}_{-1}(\widehat{\boldsymbol{\theta}})\right)\right]^{1 / 2}\right)$ e erro padrão empírico das $M$ estimativas $\left(E P_{e m p}=\left[\sum_{k=1}^{M}\left(\widehat{\boldsymbol{\theta}}_{k}-\overline{\widehat{\boldsymbol{\theta}}}\right)^{2} / M\right]^{1 / 2}\right)$.

\begin{tabular}{c|c|cc}
\hline \hline & $\overline{\widehat{\boldsymbol{\theta}}}$ & $E P(\widehat{\boldsymbol{\theta}})$ & $E P_{e m p}$ \\
\hline$\alpha$ & 0.867 & 0.040 & 0.037 \\
$\beta$ & 2.001 & 0.063 & 0.061 \\
$\sigma^{2}$ & 0.381 & 0.040 & 0.042 \\
$\lambda$ & 4.813 & 0.722 & 0.694 \\
\hline \hline
\end{tabular}

parâmetros se diferem um pouco dos valores simulados, o que pode ser devido ao alto valor do parâmetro de escala $\sigma^{2}$. Em relação ao erros padrão, tanto o empírico como o teórico, em todos os modelos os mesmos são muito similares, o que demonstra a eficiência da matriz de informação observada.

Quanto ao tempo e número de iterações necessários para a convergência, a Tabela 4.6 traz as informações para cada modelo analisado. O critério de parada adotado neste trabalho é $\left\|\boldsymbol{\theta}^{k+1}-\boldsymbol{\theta}^{k}\right\|<10^{-6}$.

Tabela 4.6: Médias de tempo e do número de iterações para cada modelo assimétrico (normal, slash, exponencial potência, t de Student generalizado e normal contaminado) nas M replicações.

\begin{tabular}{l|ccccc}
\hline \hline & \multicolumn{5}{|c}{ Modelos } \\
\cline { 2 - 6 } & NA & SLA & EPA & GtA & NCA \\
\hline Tempo médio (em segundos) & 0.76 & 17 & 34 & 32.13 & 2.66 \\
Número médio de iterações & 1236 & 1170 & 2960 & 2657 & 1140 \\
\hline \hline
\end{tabular}

\subsection{Análise de Diagnóstico}

Nesta seção serão calculadas a matriz hessiana $\left(\ddot{Q}_{\boldsymbol{\theta}}(\widehat{\boldsymbol{\theta}})\right)$ e as matrizes $\boldsymbol{\Delta}$ para análise de diagnóstico, sob o enfoque de Zhu e Lee (2001). As perturbações consideradas são ponderações de casos, perturbação no parâmetro de escala $\sigma^{2}$, perturbação no parâmetro de assimetria $\lambda$, perturbação em uma variável explicativa e perturbação na variável resposta. 
Também é calculada a matriz $\ddot{Q}_{\boldsymbol{\theta}, \mathbf{y}}(\widehat{\boldsymbol{\theta}})$ de alavancagem.

\subsubsection{A matriz hessiana}

Para obter as medidas de diagnósticos baseadas na metodologia de Zhu e Lee (2001), é necessário calcular $\ddot{Q}_{\boldsymbol{\theta}}(\widehat{\boldsymbol{\theta}})=\left.\frac{\partial^{2} Q(\boldsymbol{\theta} \mid \widehat{\boldsymbol{\theta}})}{\partial \boldsymbol{\theta} \partial \boldsymbol{\theta}^{\top}}\right|_{\boldsymbol{\theta}=\widehat{\boldsymbol{\theta}}}$, onde $\boldsymbol{\theta}=\left(\boldsymbol{\beta}^{\top}, \sigma^{2}, \lambda\right)^{\top}$. Segue de (4.24) que $\ddot{Q}_{\boldsymbol{\theta}}(\widehat{\boldsymbol{\theta}})$ tem elementos dados por

$$
\begin{aligned}
\frac{\partial^{2} Q(\boldsymbol{\theta} \mid \widehat{\boldsymbol{\theta}})}{\partial \boldsymbol{\beta} \partial \boldsymbol{\beta}^{\top}} & =-\frac{1}{\sigma^{2}} \mathbf{X}^{\top}\left(\mathbf{D}(\widehat{\boldsymbol{\kappa}})+\lambda^{2} \mathbb{I}_{n}\right) \mathbf{X} \\
\frac{\partial^{2} Q(\boldsymbol{\theta} \mid \widehat{\boldsymbol{\theta}})}{\partial \boldsymbol{\beta} \partial \sigma^{2}} & =-\frac{1}{\sigma^{4}} \mathbf{X}^{\top}\left[\mathbf{D}(\widehat{\boldsymbol{\kappa}})(\mathbf{y}-\mathbf{X} \boldsymbol{\beta})+\lambda^{2}(\mathbf{y}-\mathbf{X} \boldsymbol{\beta})-\lambda \widehat{\mathbf{t}}\right] \\
\frac{\partial^{2} Q(\boldsymbol{\theta} \mid \widehat{\boldsymbol{\theta}})}{\partial \boldsymbol{\beta} \partial \lambda} & =\frac{1}{\sigma^{2}} \mathbf{X}^{\top}(2 \lambda(\mathbf{y}-\mathbf{X} \boldsymbol{\beta})-\widehat{\mathbf{t}}) \\
\frac{\partial^{2} Q(\boldsymbol{\theta} \mid \widehat{\boldsymbol{\theta}})}{\partial \sigma^{2} \partial \sigma^{2}} & =\frac{n}{\sigma^{4}}-\frac{1}{\sigma^{6}}\left[Q_{\widehat{\mathbf{k}}}(\boldsymbol{\beta})+\widehat{\mathbf{t}}^{\top} \mathbf{1}_{n}-2 \lambda(\mathbf{y}-\mathbf{X} \boldsymbol{\beta})^{\top} \widehat{\mathbf{t}}+\lambda^{2} Q(\boldsymbol{\beta})\right] \\
\frac{\partial^{2} Q(\boldsymbol{\theta} \mid \widehat{\boldsymbol{\theta}})}{\partial \lambda^{2}} & =-\frac{1}{\sigma^{2}} Q(\boldsymbol{\beta}) \mathrm{e} \\
\frac{\partial^{2} Q(\boldsymbol{\theta} \mid \widehat{\boldsymbol{\theta}})}{\partial \sigma^{2} \partial \lambda} & =\frac{1}{\sigma^{4}}\left[\lambda Q(\boldsymbol{\beta})-(\mathbf{y}-\mathbf{X} \boldsymbol{\beta})^{\top} \widehat{\mathbf{t}}\right]
\end{aligned}
$$

onde $\widehat{\mathbf{t}^{2}}=\left({\widehat{t^{2}}}^{2}, \ldots, \widehat{t}^{2}{ }_{n}\right)^{\top}, Q(\boldsymbol{\beta})=(\mathbf{y}-\mathbf{X} \boldsymbol{\beta})^{\top}(\mathbf{y}-\mathbf{X} \boldsymbol{\beta}), Q_{\widehat{\boldsymbol{\kappa}}}(\boldsymbol{\beta})=(\mathbf{y}-\mathbf{X} \boldsymbol{\beta})^{\top} \mathbf{D}(\widehat{\boldsymbol{\kappa}})(\mathbf{y}-\mathbf{X} \boldsymbol{\beta})$ e $\mathbf{D}(\widehat{\boldsymbol{\kappa}})=\operatorname{Diag}\left(\widehat{\kappa}_{1}, \ldots, \widehat{\kappa}_{n}\right), \widehat{\kappa}_{i}$ obtidos como na Seção 4.4.1.

\subsubsection{Perturbação de ponderação de casos}

Seja $\boldsymbol{\omega}=\left(\omega_{1}, \ldots, \omega_{n}\right)^{\top}$ um vetor de dimensão $n \times 1$ com $\boldsymbol{\omega}_{0}=(1, \ldots, 1)^{\top}$. Então, a esperança da função log-verossimilhança de dados completos perturbada (função Q perturbada) pode ser escrita como

$$
Q(\boldsymbol{\theta}, \boldsymbol{\omega} \mid \widehat{\boldsymbol{\theta}})=\sum_{i=1}^{n} \omega_{i} Q_{i}(\boldsymbol{\theta} \mid \widehat{\boldsymbol{\theta}}) .
$$

Ferreira, C. S. 
Nesse caso temos $\boldsymbol{\Delta}_{\boldsymbol{\omega}_{0}}=\left.\frac{\partial^{2} Q(\boldsymbol{\theta}, \boldsymbol{\omega} \mid \widehat{\boldsymbol{\theta}})}{\partial \boldsymbol{\theta} \partial \boldsymbol{\omega}^{\top}}\right|_{\boldsymbol{\omega}=\boldsymbol{\omega}_{0}}=\left(\boldsymbol{\Delta}_{\boldsymbol{\beta}}^{\top}, \boldsymbol{\Delta}_{\sigma^{2}}^{\top}, \boldsymbol{\Delta}_{\lambda}^{\top}\right)^{\top}$ com elementos dados por

$$
\begin{aligned}
\boldsymbol{\Delta}_{\boldsymbol{\beta}}= & \frac{1}{\sigma^{2}} \mathbf{X}^{\top}\left(\mathbf{D}(\widehat{\boldsymbol{\kappa}}) \mathbf{D}(\mathbf{y}-\mathbf{X} \boldsymbol{\beta})-\lambda \mathbf{D}(\widehat{\mathbf{t}})+\lambda^{2} \mathbf{D}(\mathbf{y}-\mathbf{X} \boldsymbol{\beta})\right), \\
\boldsymbol{\Delta}_{\sigma^{2}}= & -\frac{1}{\sigma^{2}} \mathbf{1}_{n}^{\top}+\frac{1}{2 \sigma^{4}}\left[(\mathbf{y}-\mathbf{X} \boldsymbol{\beta})^{\top}\left(\mathbf{D}(\widehat{\boldsymbol{\kappa}})+\lambda^{2} \mathbb{I}_{n}\right) \mathbf{D}(\mathbf{y}-\mathbf{X} \boldsymbol{\beta})\right. \\
& \left.+\widehat{\mathbf{t}}^{\top}-2 \lambda(\mathbf{y}-\mathbf{X} \boldsymbol{\beta})^{\top} \mathbf{D}(\widehat{\mathbf{t}})\right] \mathrm{e} \\
\boldsymbol{\Delta}_{\lambda}= & \frac{1}{\sigma^{2}}(\mathbf{y}-\mathbf{X} \boldsymbol{\beta})^{\top}[\mathbf{D}(\widehat{\mathbf{t}})-\lambda \mathbf{D}(\mathbf{y}-\mathbf{X} \boldsymbol{\beta})] .
\end{aligned}
$$

\subsubsection{Perturbação do parâmetro de escala}

Esse esquema de perturbação é introduzido considerando o modelo

$$
y_{i \omega_{i}} \sim \operatorname{MENA}\left(\mathbf{x}_{i}^{\top} \boldsymbol{\beta}, \omega_{i}^{-1} \sigma^{2}, \lambda\right), \omega_{i}>0, i=1, \ldots, n .
$$

A função $Q$ perturbada é da forma

$$
\begin{aligned}
Q(\boldsymbol{\theta}, \boldsymbol{\omega} \mid \widehat{\boldsymbol{\theta}}) \propto & -\sum_{i=1}^{n} \log \frac{\sigma^{2}}{\omega_{i}}-\frac{1}{2 \sigma^{2}} \sum_{i=1}^{n} \omega_{i}{\widehat{t^{2}}}_{i}-\frac{1}{2 \sigma^{2}} \sum_{i=1}^{n} \omega_{i}\left(\widehat{\kappa}_{i}+\lambda^{2}\right)\left(y_{i}-\mathbf{x}_{i}^{\top} \boldsymbol{\beta}\right)^{2} \\
& +\frac{\lambda}{\sigma^{2}} \sum_{i=1}^{n} \omega_{i} \widehat{t}_{i}\left(y_{i}-\mathbf{x}_{i}^{\top} \boldsymbol{\beta}\right) .
\end{aligned}
$$

Sob esse esquema de perturbação o modelo não-perturbado segue considerando $\boldsymbol{\omega}_{0}=$ $(1, \ldots, 1)^{\top}$ e a matriz $\boldsymbol{\Delta}_{\boldsymbol{\omega}_{0}}$ é dada por

$$
\boldsymbol{\Delta}_{\boldsymbol{\omega}_{0}}=\frac{1}{\sigma^{2}}\left[\begin{array}{l}
\mathbf{X}^{\top}\left(\mathbf{D}(\widehat{\boldsymbol{\kappa}})+\lambda^{2} \mathbb{I}_{n}\right) \mathbf{D}(\mathbf{y}-\mathbf{X} \boldsymbol{\beta})-\lambda \mathbf{X}^{\top} \mathbf{D}(\widehat{\mathbf{t}}) \\
\frac{1}{2 \sigma^{2}}\left[(\mathbf{y}-\mathbf{X} \boldsymbol{\beta})^{\top}\left(\mathbf{D}(\widehat{\boldsymbol{\kappa}})+\lambda^{2} \mathbb{I}_{n}\right) \mathbf{D}(\mathbf{y}-\mathbf{X} \boldsymbol{\beta})-2 \lambda(\mathbf{y}-\mathbf{X} \boldsymbol{\beta})^{\top} \mathbf{D}(\widehat{\mathbf{t}})+\widehat{\mathbf{t}}^{\top}\right. \\
(\mathbf{y}-\mathbf{X} \boldsymbol{\beta})^{\top}(\mathbf{D}(\widehat{\mathbf{t}})-\lambda \mathbf{D}(\mathbf{y}-\mathbf{X} \boldsymbol{\beta}))
\end{array}\right] .
$$

\subsubsection{Perturbação do parâmetro de assimetria}

Esse esquema de perturbação é introduzido considerando o modelo

$$
y_{i} \sim \operatorname{MENA}\left(\mathbf{x}_{i}^{\top} \boldsymbol{\beta}, \sigma^{2}, \lambda \omega_{i}\right), \omega_{i} \in \mathbb{R}, i=1, \ldots, n .
$$


Sendo $\boldsymbol{\omega}=\left(\omega_{1}, \ldots, \omega_{n}\right)^{\top}$, a função t Q perturbada é da forma

$$
\begin{aligned}
Q(\boldsymbol{\theta}, \boldsymbol{\omega} \mid \widehat{\boldsymbol{\theta}}) \propto & -n \log \sigma^{2}-\frac{1}{2 \sigma^{2}} Q_{\widehat{\boldsymbol{\kappa}}}(\boldsymbol{\beta}) \\
& -\frac{1}{2 \sigma^{2}} \sum_{i=1}^{n}\left(\widehat{t}^{2}{ }_{i}-2 \lambda \omega_{i} \widehat{t}_{i}\left(y_{i}-\mathbf{x}_{i}^{\top} \boldsymbol{\beta}\right)+\lambda^{2} \omega_{i}^{2}\left(y_{i}-\mathbf{x}_{i}^{\top} \boldsymbol{\beta}\right)^{2}\right) .
\end{aligned}
$$

Sob esse esquema de perturbação o modelo não-perturbado segue considerando $\boldsymbol{\omega}_{0}=$ $(1, \ldots, 1)^{\top}$ e a matriz $\boldsymbol{\Delta}_{\boldsymbol{\omega}_{0}}$ é dada por

$$
\boldsymbol{\Delta}_{\boldsymbol{\omega}_{0}}=\frac{1}{\sigma^{2}}\left[\begin{array}{l}
\lambda \mathbf{X}^{\top}(2 \lambda \mathbf{D}(\mathbf{y}-\mathbf{X} \boldsymbol{\beta})-\mathbf{D}(\widehat{\mathbf{t}})) \\
\frac{\lambda}{\sigma^{2}}(\mathbf{y}-\mathbf{X} \boldsymbol{\beta})^{\top}[\lambda \mathbf{D}(\mathbf{y}-\mathbf{X} \boldsymbol{\beta})-\mathbf{D}(\widehat{\mathbf{t}})] \\
(\mathbf{y}-\mathbf{X} \boldsymbol{\beta})^{\top}(\mathbf{D}(\widehat{\mathbf{t}})-2 \lambda \mathbf{D}(\mathbf{y}-\mathbf{X} \boldsymbol{\beta}))
\end{array}\right] .
$$

\subsubsection{Perturbação em uma variável explicativa}

Nesse caso o interesse está em perturbar uma variável explicativa específica permitindo, por exemplo, detectar possíveis maus condicionamentos de alguma coluna da matriz de desenho (Belsley, 1991). Sob essa condição, tem-se a seguinte o esquema de perturbação aditiva em uma variável explicativa $\left(X_{v}\right)$

$$
x_{i v}(\boldsymbol{\omega})=x_{i v}+S_{v} \omega_{i}, \quad v \in\{1, \ldots, p\}, \omega_{i} \in \mathbb{R}, i=1, \ldots, n .
$$

onde $S_{v}$ é o desvio padrão da variável explicativa $X_{v}$. Nesse caso, $\boldsymbol{\omega}_{0}=\mathbf{0}$ e a função $Q$ perturbada é dada por

$$
\begin{aligned}
Q(\boldsymbol{\theta}, \boldsymbol{\omega} \mid \widehat{\boldsymbol{\theta}}) \propto & -n \log \sigma^{2}-\frac{1}{2 \sigma^{2}}\left(\mathbf{y}-\mathbf{X}_{\boldsymbol{\omega}} \boldsymbol{\beta}\right)^{\top}\left(\mathbf{D}(\widehat{\boldsymbol{\kappa}})+\lambda^{2} \mathbb{I}_{n}\right)\left(\mathbf{y}-\mathbf{X}_{\boldsymbol{\omega}} \boldsymbol{\beta}\right) \\
& -\frac{1}{2 \sigma^{2}} \widehat{\mathbf{t}}^{\top} \mathbf{1}_{n}+\frac{\lambda}{\sigma^{2}}\left(\mathbf{y}-\mathbf{X}_{\boldsymbol{\omega}} \boldsymbol{\beta}\right)^{\top} \widehat{\mathbf{t}}
\end{aligned}
$$

Segue que a matriz $\boldsymbol{\Delta} \boldsymbol{\omega}_{0}$ tem a forma

$$
\boldsymbol{\Delta}_{\boldsymbol{\omega}_{0}}=\frac{S_{v}}{\sigma^{2}}\left[\begin{array}{c}
\left(\mathbf{0}_{v}^{1}(\mathbf{y}-\mathbf{X} \boldsymbol{\beta})^{\top}-\beta_{v} \mathbf{X}^{\top}\right)\left[\mathbf{D}(\widehat{\boldsymbol{\kappa}})+\lambda^{2} \mathbb{I}_{n}\right]-\lambda \mathbf{0}_{v}^{1} \widehat{\mathbf{t}}^{\top} \\
\frac{\beta_{v}}{\sigma^{2}}\left((\mathbf{y}-\mathbf{X} \boldsymbol{\beta})^{\top} \mathbf{D}(\widehat{\boldsymbol{\kappa}})+\lambda \widehat{\mathbf{t}}^{\top}+\lambda^{2}(\mathbf{y}-\mathbf{X} \boldsymbol{\beta})^{\top}\right) \\
\beta_{v}\left(-\widehat{\mathbf{t}}^{\top}+2 \lambda(\mathbf{y}-\mathbf{X} \boldsymbol{\beta})^{\top}\right)
\end{array}\right]
$$

onde $\mathbf{0}_{v}^{1}$ denota um vetor de zeros de tamanho $p \times 1$ com um 1 na $v$-ésima posição.

Ferreira, C. S. 


\subsubsection{Perturbação na variável resposta}

A perturbação em $\mathbf{y}=\left(y_{1}, \ldots, y_{n}\right)^{\top}$ é introduzida substituindo $y_{i}$ por $y_{\omega_{i}}=y_{i}+\omega_{i} S_{y}$, onde $S_{y}$ é o desvio padrão de $\mathbf{y}$. Neste caso $\boldsymbol{\omega}_{0}=\mathbf{0}$ e

$$
\begin{aligned}
Q(\boldsymbol{\theta}, \boldsymbol{\omega} \mid \widehat{\boldsymbol{\theta}}) \propto & -n \log \sigma^{2}-\frac{1}{2 \sigma^{2}}(\mathbf{y} \boldsymbol{\omega}-\mathbf{X} \boldsymbol{\beta})^{\top}\left(\mathbf{D}(\widehat{\boldsymbol{\kappa}})+\lambda^{2} \mathbb{I}_{n}\right)(\mathbf{y} \boldsymbol{\omega}-\mathbf{X} \boldsymbol{\beta}) \\
& -\frac{1}{2 \sigma^{2}} \widehat{\mathbf{t}}^{\top} \mathbf{1}_{n}+\frac{\lambda}{\sigma^{2}}(\mathbf{y} \boldsymbol{\omega}-\mathbf{X} \boldsymbol{\beta})^{\top} \widehat{\mathbf{t}} .
\end{aligned}
$$

Segue que a matriz $\boldsymbol{\Delta}_{\boldsymbol{\omega}_{0}}$ tem a forma

$$
\boldsymbol{\Delta}_{\boldsymbol{\omega}_{0}}=\frac{S_{y}}{\sigma^{2}}\left[\begin{array}{l}
\mathbf{X}^{\top}\left(\mathbf{D}(\widehat{\boldsymbol{\kappa}})+\lambda^{2} \mathbb{I}_{n}\right) \\
\frac{1}{\sigma^{2}}\left((\mathbf{y}-\mathbf{X} \boldsymbol{\beta})^{\top} \mathbf{D}(\widehat{\boldsymbol{\kappa}})+\lambda^{2}(\mathbf{y}-\mathbf{X} \boldsymbol{\beta})^{\top}-\lambda \widehat{\mathbf{t}}^{\top}\right) \\
\widehat{\mathbf{t}}^{\top}-2 \lambda(\mathbf{y}-\mathbf{X} \boldsymbol{\beta})^{\top}
\end{array}\right] .
$$

\subsubsection{Alavancagem generalizada}

A matriz $\ddot{Q}_{\boldsymbol{\theta}, \mathbf{y}}(\widehat{\boldsymbol{\theta}})$ é como em (4.30) sem o termo $S_{y}$ e $\ddot{Q}_{\boldsymbol{\theta}}(\widehat{\boldsymbol{\theta}})$ é dada na Seção 4.7.1.

Para o modelo normal assimétrico, tem-se que $\boldsymbol{\mu}=E[\mathbf{Y}]=\mathbf{X} \boldsymbol{\beta}+c \sigma \rho \mathbf{1}_{n}$, onde $c=\sqrt{2 / \pi}$ e $\rho=\frac{\lambda}{\sqrt{1+\lambda^{2}}}$. Deste modo, a matriz $D_{\boldsymbol{\theta}}: n \times(p+2)$ é da forma

$$
D_{\boldsymbol{\theta}}=\left[\mathbf{X}, \frac{c \rho}{2 \sigma} \mathbf{1}_{n}, \frac{c \sigma}{\left(1+\lambda^{2}\right)^{3 / 2}} \mathbf{1}_{n}\right]
$$

- Modelo t de Student generalizado normal assimétrico:

$$
D_{\boldsymbol{\theta}}^{\top}=\left[\begin{array}{c}
\mathbf{X}^{\top} \\
\frac{\lambda \gamma^{1 / 2} \Gamma\left(\frac{\nu-1}{2}\right)}{2 \sigma \pi^{1 / 2} \Gamma(\nu / 2)} E\left(\left(V+\lambda^{2}\right)^{-1 / 2}\right) \mathbf{1}_{n}^{\top} \\
\frac{\sigma \gamma^{1 / 2} \Gamma\left(\frac{\nu-1}{2}\right)}{\pi^{1 / 2} \Gamma(\nu / 2)}\left[E\left(\left(V+\lambda^{2}\right)^{-1 / 2}\right)-\lambda^{2} E\left(\left(V+\lambda^{2}\right)^{-3 / 2}\right)\right] \mathbf{1}_{n}^{\top}
\end{array}\right]
$$

onde $V \sim \operatorname{Gama}\left(\frac{\nu-1}{2}, \frac{\gamma}{2}\right)$. 
- Modelo slash assimétrico:

$$
D_{\boldsymbol{\theta}}^{\top}=\left[\begin{array}{c}
\mathbf{X}^{\top} \\
\frac{\nu \lambda}{\sigma(2 \pi)^{1 / 2}(\nu-1 / 2)} E\left(\left(V+\lambda^{2}\right)^{-1 / 2}\right) \mathbf{1}_{n}^{\top} \\
\frac{c \sigma \nu}{(\nu-1 / 2)}\left[E\left(\left(V+\lambda^{2}\right)^{-1 / 2}\right)-\lambda^{2} E\left(\left(V+\lambda^{2}\right)^{-3 / 2}\right)\right] \mathbf{1}_{n}^{\top}
\end{array}\right]
$$

onde $V \sim h(v ; \nu-1 / 2), h(u ; \nu)=\nu u^{\nu-1} \mathbf{I}_{(0,1)}(u)$.

- Modelo normal contaminado assimétrico:

$$
D_{\boldsymbol{\theta}}^{\top}=\left[\begin{array}{c}
\mathbf{X}^{\top} \\
\frac{\lambda}{\sigma(2 \pi)^{1 / 2}}\left(\frac{\nu}{\left(\gamma\left(\gamma+\lambda^{2}\right)\right)^{1 / 2}}+\frac{1-\gamma}{\left(1+\lambda^{2}\right)^{1 / 2}}\right) \mathbf{1}_{n}^{\top} \\
c \sigma\left(\frac{\nu \gamma^{1 / 2}}{\left(\gamma+\lambda^{2}\right)^{1 / 2}}+\frac{1-\gamma}{\left(1+\lambda^{2}\right)^{1 / 2}}\right) \mathbf{1}_{n}^{\top}
\end{array}\right]
$$

- Modelo exponencial potência assimétrico: $D_{\boldsymbol{\theta}}=\left[\begin{array}{lll}D_{\boldsymbol{\beta}}, & D_{\sigma^{2}}, & D_{\lambda}\end{array}\right]$, onde

$$
\begin{aligned}
& D_{\boldsymbol{\beta}}=-\frac{2 k_{\nu}}{\lambda}\left(\sigma E_{Z}\left[Z \exp \left\{-\frac{Z^{2 \nu}}{2 \lambda^{2 \nu}}\right\}\right] \mathbb{I}_{n}+\lambda E_{Z}\left[\exp \left\{-\frac{Z^{2 \nu}}{2 \lambda^{2 \nu}}\right\}\right] \mathbf{D}(\mathbf{X} \boldsymbol{\beta})\right) \mathbf{X}+\frac{2 \nu k_{\nu}}{\sigma^{2 \nu}} \mathbf{D}(\mathbf{J}) \mathbf{X} \\
& D_{\sigma^{2}}=-\frac{k_{\nu}}{\lambda^{2} \sigma}\left(\sigma E_{Z}\left[Z^{2} \exp \left\{-\frac{Z^{2 \nu}}{2 \lambda^{2 \nu}}\right\}\right] \mathbf{1}_{n}+\lambda E_{Z}\left[Z \exp \left\{-\frac{Z^{2 \nu}}{2 \lambda^{2 \nu}}\right\}\right] \mathbf{X} \boldsymbol{\beta}\right)+\frac{\nu k_{\nu}}{\sigma^{2(\nu+1)}} \mathbf{K} \\
& D_{\lambda}=\frac{2 \sigma k_{\nu}}{\lambda^{3}}\left(\sigma E_{Z}\left[Z^{2} \exp \left\{-\frac{Z^{2 \nu}}{2 \lambda^{2 \nu}}\right\}\right] \mathbf{1}_{n}+\lambda E_{Z}\left[Z \exp \left\{-\frac{Z^{2 \nu}}{2 \lambda^{2 \nu}}\right\}\right] \mathbf{X} \boldsymbol{\beta}\right)
\end{aligned}
$$

onde $\mathbf{J}=\left(J_{1}, \ldots, J_{n}\right), J_{i}=\int_{-\infty}^{+\infty} \operatorname{sinal}\left(u-\mathbf{x}_{i}^{\top} \boldsymbol{\beta}\right) u\left|u-\mathbf{x}_{i}^{\top} \boldsymbol{\beta}\right|^{2 \nu-1} e^{-\frac{d_{i}^{\nu}}{2}} \Phi\left(\frac{\lambda\left(u-\mathbf{x}_{i}^{\top} \boldsymbol{\beta}\right)}{\sigma}\right) d u$, $\mathbf{K}=\left(K_{1}, \ldots, K_{n}\right)^{\top}, K_{i}=\int_{-\infty}^{+\infty} \operatorname{sinal}\left(u-\mathbf{x}_{i}^{\top} \boldsymbol{\beta}\right) u\left(u-\mathbf{x}_{i}^{\top} \boldsymbol{\beta}\right)^{2 \nu} e^{-\frac{d_{i}^{\nu}}{2}} \Phi\left(\frac{\lambda\left(u-\mathbf{x}_{i}^{\top} \boldsymbol{\beta}\right)}{\sigma}\right) d u$,

$d_{i}=\frac{\left(u-\mathbf{x}_{i}^{\top} \boldsymbol{\beta}\right)^{2}}{\sigma^{2}}, k_{\nu}=\frac{\nu}{2^{1 / 2 \nu} \Gamma(1 / 2 \nu)}, \mathbf{1}_{n}$ é um vetor de 1 's de tamanho $n, \mathbb{I}_{n}$ é a matriz identidade de ordem $n$ e $Z \sim N(0,1)$.

\subsection{Estudo de Simulação}

Nessa seção são apresentadas duas aplicações ilustrando o uso das distribuições $N A$, GtA, SLA, NCA e EPA, em estudos simulados.

Como ilustração, será usado um estudo de simulação de pequena escala para estudar o comportamento de dois estimadores, a média e a mediana amostrais. Considera-se uma distribuição normal padrão assimétrica $N A(3)$, uma distribuição t-normal assimétrica $G t A(3 ; 2,2)$, uma distribuição slash assimétrica $S L A(3 ; 0.5)$, uma distribuição exponen- 

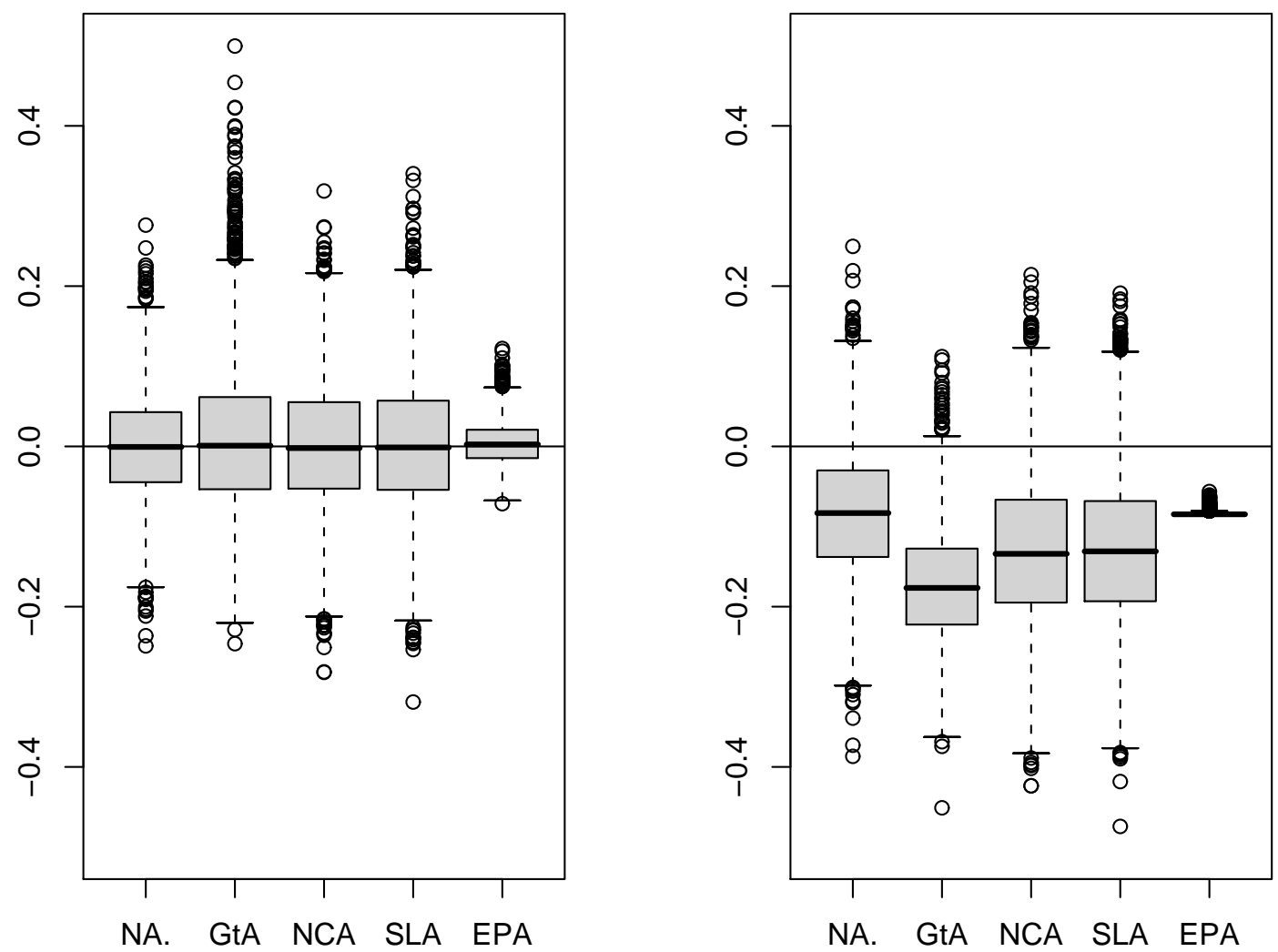

Figura 4.2: Boxplots das médias amostrais (painel à esquerda) e medianas amostrais (painel à direita) baseadas em 500 amostras de tamanho $\mathrm{n}=100$ das cinco distribuições padrão $(\mu=0$ e $\left.\sigma^{2}=1\right)$ : $N A(3) ; G t A(3 ; 3,3) ; N C A(3 ; 0.5,0.5) ; S L A(3 ; 1)$, e $E P A(3 ; 0.5)$. As respectivas médias foram ajustadas para zero.

cial potência assimétrica $E P A(3 ; 0.5)$ e uma distribuição normal contaminada assimétrica $N C A(3 ; 0.9,0.1)$. A média de todas as distribuições é ajustada para zero, logo as cinco distribuições são comparáveis. Assim, esse grupo representa cinco distribuições com a mesma média mas com diferentes comportamentos de cauda e assimetria. Foram simuladas 500 amostras de tamanho $n=100$ de cada uma das cinco distribuições e para cada amostra, foram calculadas a média e a mediana amostrais e os resultados são apresentados em gráficos de caixa (boxplot) para cada distribuição (Figura 4.2). No painel à esquerda, observa-se que todos os boxplots das médias estimadas são centrados em torno de 0 , porém têm maior variabilidade nas distribuições de caudas mais pesadas (t-normal, normal contaminada e slash 
assimétricas). No painel à direita, os boxplots das medianas amostrais têm uma variabilidade levemente maior que os boxplots das médias amostrais para as distribuições normal, normal contaminada e slash assimétricas, mas têm uma variabilidade menor para as distribuições t-normal e exponencial potência assimétricas.

Isso indica que a mediana é um estimador eficiente da locação de distribuições assimétricas de caudas leves. Por outro lado, o estimador da mediana se torna viesado quando na presença de assimetria e caudas mais pesadas.

\subsection{Aplicação}

Nesta seção analisamos o conjunto de dados de variáveis biomédicas do Instituto de Esportes da Austrália (AIS). Um subconjunto desses dados foi previamente analisado em Azzalini e Capitanio (2003).

Define-se o modelo

$$
\begin{aligned}
s s f_{i} & =\alpha+\beta \times b_{f a t_{i}}+\sigma \varepsilon_{i} \\
\varepsilon_{i} & \stackrel{i i d}{\sim} \operatorname{MENA}(\lambda ; H), i=1, \ldots, 202,
\end{aligned}
$$

onde $b f a t_{i}$ é a porcentagem de gordura corporal e $s s f_{i}$ é a soma de manchas na pele do i-ésimo indivíduo na amostra. Arellano-Valle et al. (2005) ajustaram um modelo com erro de medição normal assimétrico para esses dados e notaram um forte relacionamento entre as variáveis e também uma assimetria positiva nos resíduos.

As distribuições normal, t-normal, slash, normal contaminada e exponencial potência assimétricas são aplicadas para ajustar os dados. Estimativas resultantes são dadas na Tabela 4.7. Os erros padrão estimados foram calculados usando a matriz de informação observada apresentada na Seção 4.5. O critério de informação de Akaike $(A I C=-2 \ell(\widehat{\boldsymbol{\theta}}) / n+2 p / n)$ e o critério de informação bayesiana $(B I C=-2 \ell(\widehat{\boldsymbol{\theta}}) / n+p \log (n) / n)$ são utilizados para fins de comparação de ajuste entre os modelos de misturas assimétricos. O critério de Informação de Schwarz (ou equivalentemente a função log-verossimilhança) foi utilizado para escolher entre os melhores valores de $\nu$ e $\gamma$ para cada modelo, segundo recomendação de Fernández e Steel (1999). Para o modelo slash assimétrico foi tomado um conjunto de 100 valores $\nu=0.5: 20$; para o modelo t de Student generalizado normal assimétrico foi tomado um conjunto de 


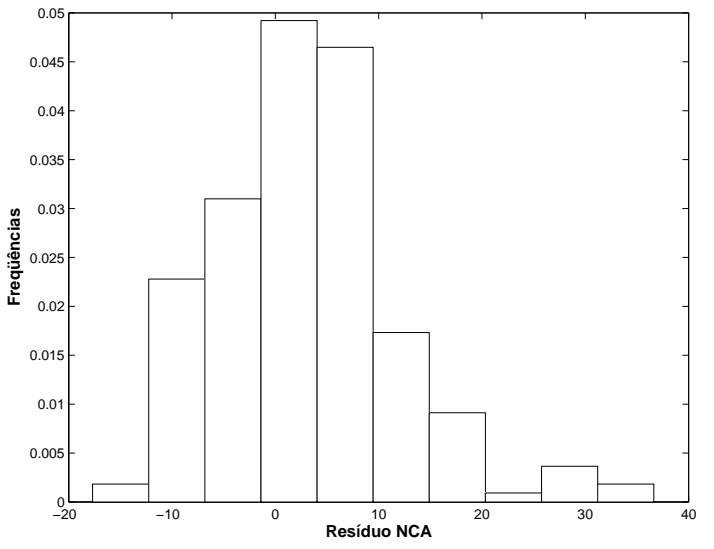

(a)

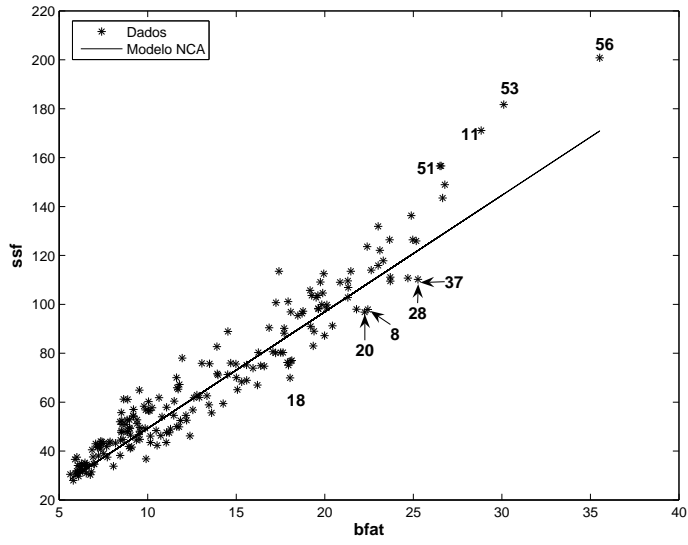

(b)

Figura 4.3: Conjunto de dados AIS. (a) Histograma do resíduo do modelo normal contaminado ajustado e (b) gráfico de dispersão $b f a t \times s s f$, juntamente com reta ajustada e pontos influentes.

valores $100 \times 100$ para $\nu, \gamma=0.5: 10$; para o modelo exponencial potência assimétrico foi tomado um conjunto de 100 valores para $\nu=0.1: 1$; para o modelo normal contaminado assimétrico foi tomado um conjunto de valores $100 \times 100$ para $\nu, \gamma=0.1: 1$.

Assim, de acordo com a Tabela 4.7, a distribuição normal contaminada fornece o melhor ajuste em relação às demais (somente esta apresentou todos os pontos no interior do envelope, Figura 4.4). Com relação aos parâmetros estimados, percebe-se que os interceptos para os modelos t de Student normal assimétrico e normal contaminado assimétrico se diferem dos interceptos das demais distribuições, sendo que para a distribuição normal contaminada, esse parâmetros pode ser removido do modelo (erro padrão superior à estimativa). Por último, percebe-se os modelos com ajustes mais satisfatórios apresentam menores valores das estimativas dos parâmetros.

Substituindo as estimativas MV $\boldsymbol{\theta}$ na distância de Mahalanobis $d_{i}=\frac{\left(y_{i}-\mathbf{x}_{i}^{\top} \boldsymbol{\beta}\right)^{2}}{\sigma^{2}}$, gráficos de envelope são apresentados na Figura 4.4 (linhas representam o percentil-5, a média e o percentil-95 de 100 pontos simulados para cada observação). Os gráficos fornecem evidência (aliado ao critério da função log-verossimilhança, Tabela 4.7) de que a distribuição normal contaminada proporciona melhor ajuste para os dados.

Medidas de diagnósticos são construídas para as perturbações de ponderação de casos, na escala, no parâmetro de assimetria $\lambda$, na variável resposta e perturbação na variável explicativa, e também alavanca e influência global ( $D i$ e $L D i$ ) para o modelo dado em (4.32). 
Tabela 4.7: EMV, AIC e BIC dos 5 modelos ajustados ao conjunto de dados AIS. Os valores entre parênteses são os erros padrão estimados assintóticos (obtidos através da matriz de informação de Fisher observada).

\begin{tabular}{cccccc}
\hline \hline & & & & & \\
& NA & SLA & EPA & GtA & NCA \\
\hline Parameter & Estimativa & Estimativa & Estimativa & Estimativa & Estimativa \\
\hline$\alpha$ & $-5.08(1.57)$ & $-5.02(1.58)$ & $-4.60(1.58)$ & $-2.02(1.88)$ & $-0.42(1.35)$ \\
$\beta$ & $4.88(0.14)$ & $4.88(0.14)$ & $4.86(0.14)$ & $4.78(0.14)$ & $4.77(0.10)$ \\
$\sigma^{2}$ & $143.97(32.76)$ & $134.25(30.72)$ & $129.88(29.82)$ & $90.59(27.01)$ & $64.64(11.42)$ \\
$\lambda$ & $1.64(0.61)$ & $1.58(0.59)$ & $1.55(0.31)$ & $1.07(0.58)$ & $0.70(0.30)$ \\
\hline$\nu$ & - & 15.67 & 0.95 & 7.88 & 0.21 \\
$\gamma$ & - & - & - & 7.88 & 0.17 \\
\hline$\ell(\widehat{\theta})$ & -723.27 & -723.51 & -723.18 & -721.97 & -720.83 \\
AIC & 7.20 & 7.20 & 7.20 & 7.19 & 7.18 \\
BIC & 7.21 & 7.21 & 7.21 & 7.19 & 7.18 \\
\hline \hline
\end{tabular}

Apresenta-se neste capítulo somente os gráficos para a distribuição normal contaminada, que propiciou o melhor ajuste. Os gráficos para as outras distribuições são expostos na Seção C.2 do Apêndice C.

Percebe-se que, pelos gráficos de influência local, o número de pontos possivelmente influentes é menor no modelo de mistura de escala normal contaminada, distribuição com o melhor ajuste para os dados. Ainda, os pontos influentes são bem caracterizados para esta distribuição. Pensando no modelo $Y_{i} \mid \mathbf{x}_{i} \sim \operatorname{MENA}\left(\mathbf{x}_{i}^{\top} \boldsymbol{\beta}, \sigma^{2}, \lambda, H\right)$, os pontos potencialmente influentes sob as perturbações de ponderação de casos e perturbação no parâmetro de escala, além das perturbações nas variáveis resposta e explicativa, são aqueles localizados na cauda leve da distribuição de $\varepsilon_{i}$. Por outro lado, são os pontos fora do subespaço gerado pelas colunas de $\mathbf{X}$ os mais influentes sob perturbação no parâmetro de assimetria.

A metodologia de alavancagem detectou o ponto 56 em todos os modelos assimétricos, um ponto de alavanca, sendo que o modelo normal detectou outros além deste.

A análise de influência global mostrou que a medida $L D i^{c}$ é muito sensível a pontos abaixo da reta estimada e também pontos de alavanca. Por outro lado, o gráfico para $D i^{c}$ identificou apenas um ponto (18), localizado abaixo da reta.

Ferreira, C. S. 


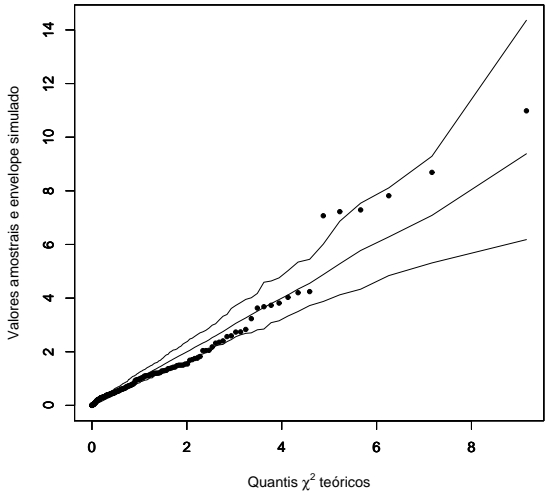

(a)

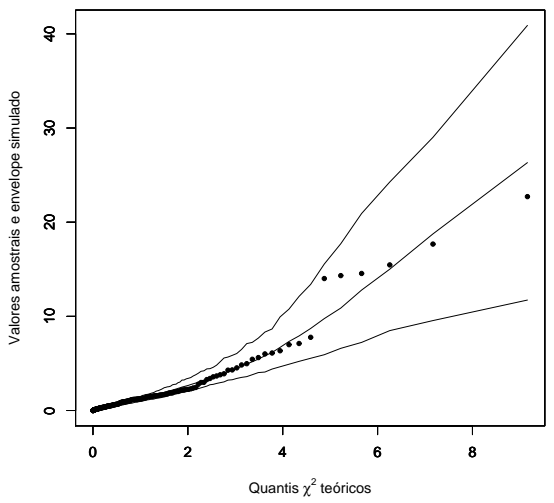

(c)

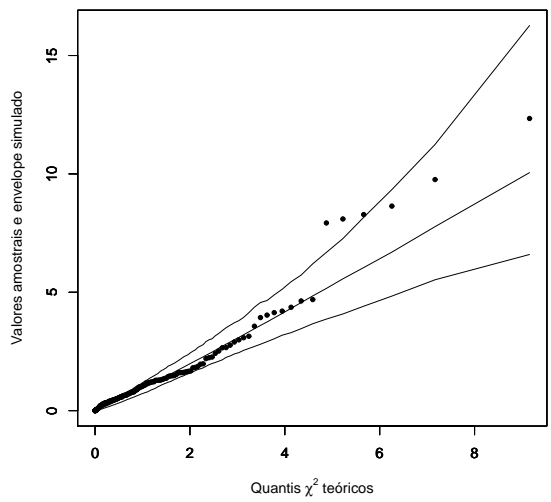

(e)

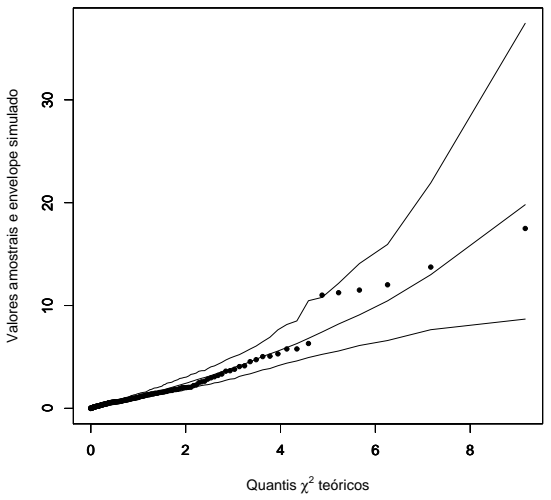

(b)

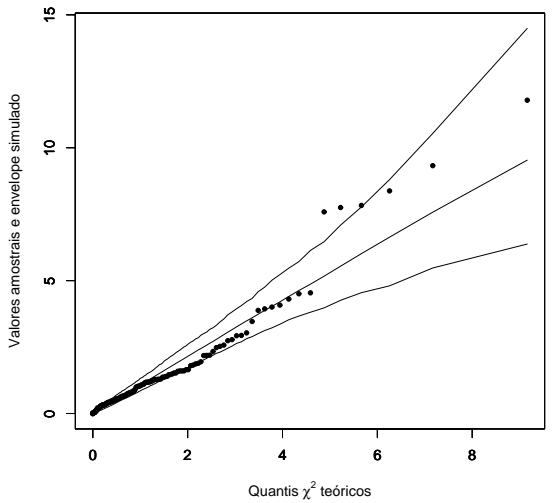

(d)

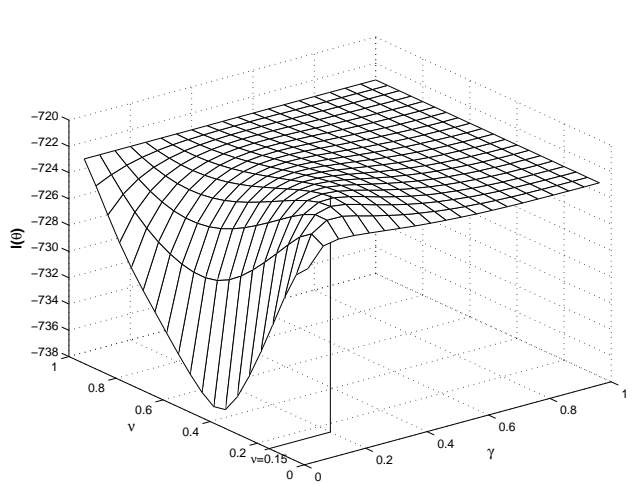

(f)

Figura 4.4: Conjunto de dados AIS. Gráficos Q-Q e envelope simulado: (a) modelo normal assimétrico, (b) modelo t-normal assimétrico, (c) modelo normal contaminado assimétrico, (d) modelo slash assimétrico, (e) modelo exponencial potência assimétrico e (f) função logverossimilhança perfilada para o modelo normal contaminado assimétrico.

Ferreira, C. S. 


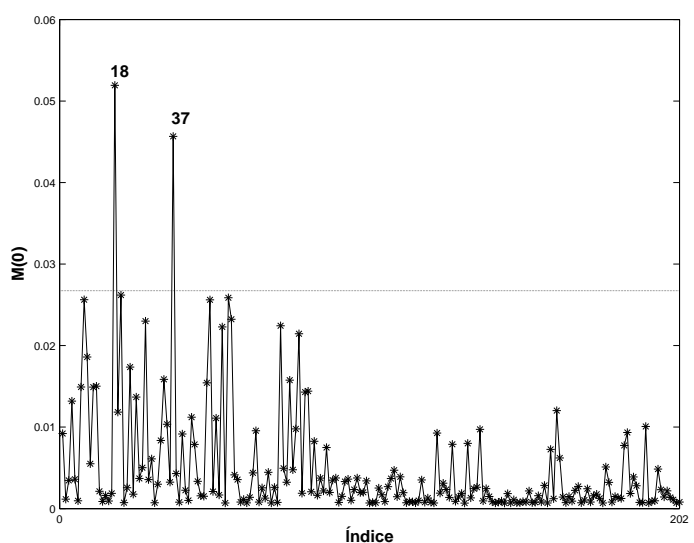

(a)

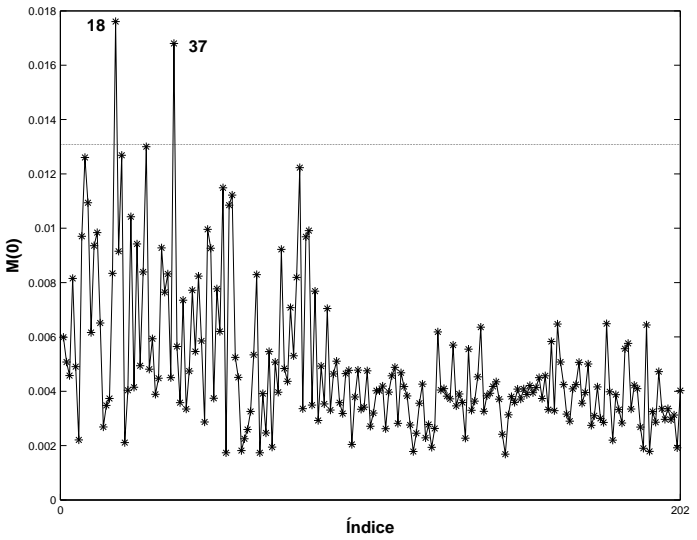

(c)

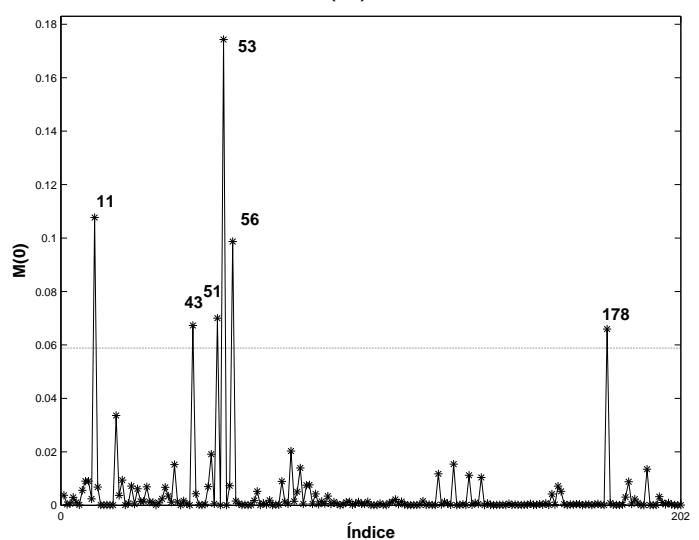

(e)

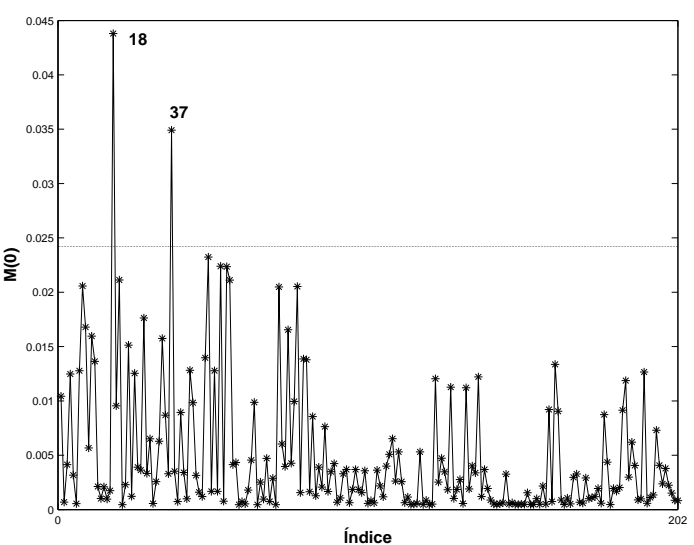

(b)

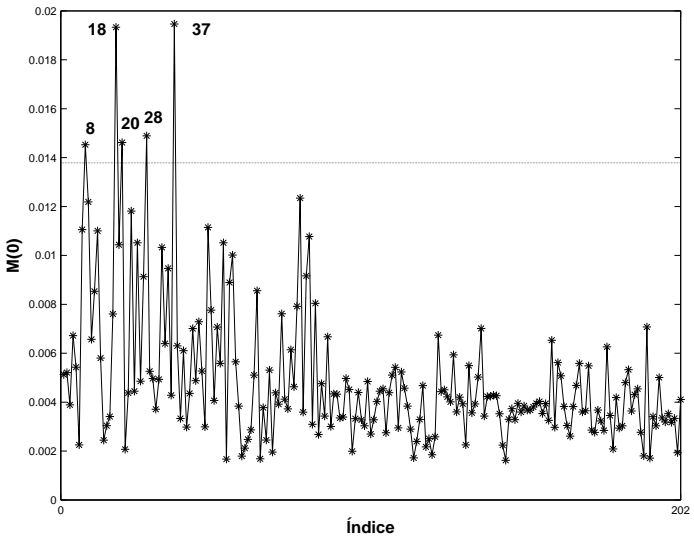

(d)

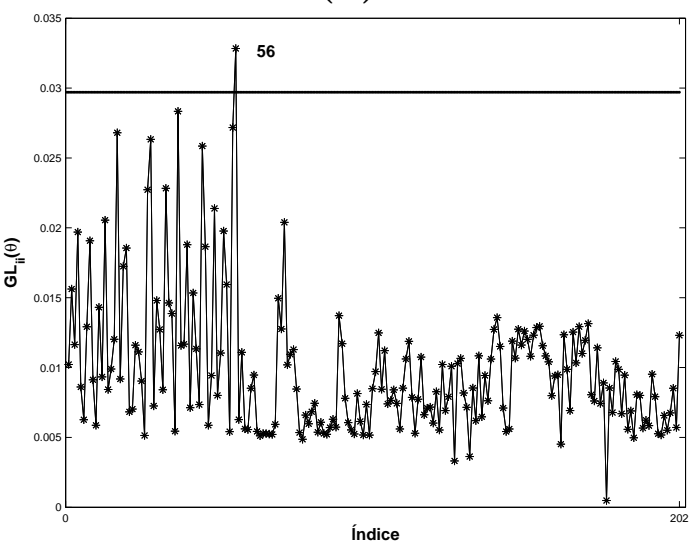

(f)

Figura 4.5: Diagnóstico de influência para os dados AIS, sob o modelo normal contaminado assimétrico. (a) ponderação de casos, (b) perturbação na escala, (c) perturbação na variável resposta, (d) perturbação na variável explicativa, (e) perturbação no parâmetro de assimetria e (f) alavanca. Linhas horizontais são os pontos de corte $\left(c^{*}=3\right)$. 


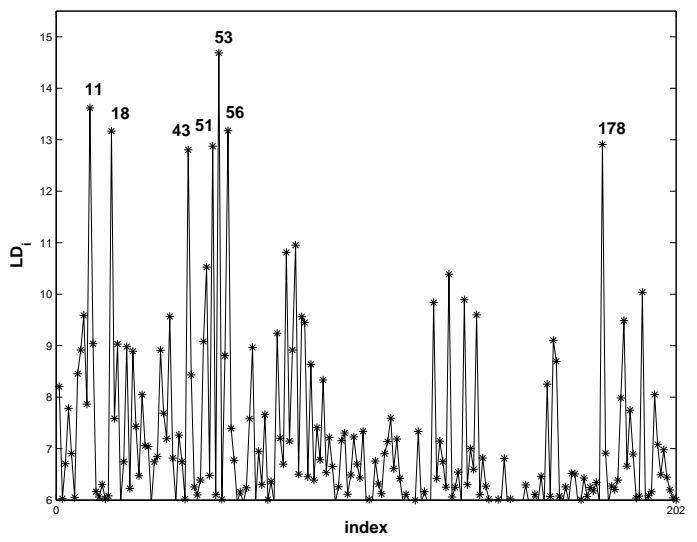

(a)

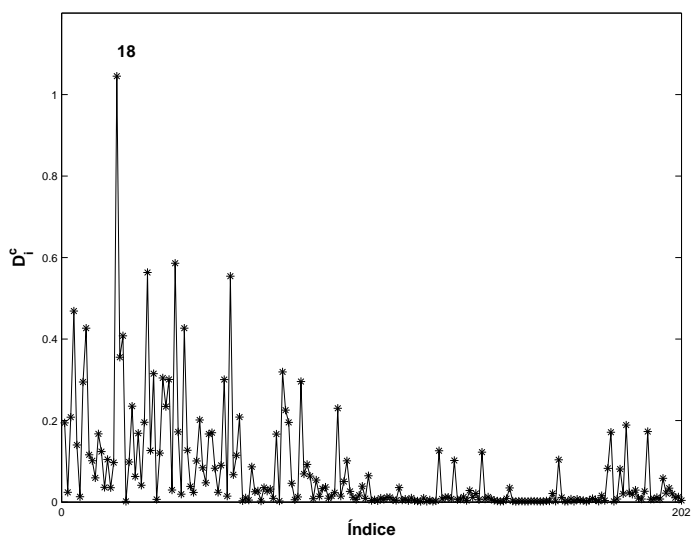

(b)

Figura 4.6: Influência global para o conjunto de dados AIS, sob o modelo normal contaminado. (a) $L D i_{c}$ e (b) $D i_{c}$. 


\section{$5 \quad$ Modelo Linear Misto Assimétrico}

\subsection{Introdução}

Modelos Lineares Mistos (MLM) são bastante utilizados para analisar dados de medidas repetidas, dados agrupados e dados longitudinais, entre outros, sendo de grande aplicabilidade em pesquisas de diversas áreas como agricultura, biologia, economia, geofísica e ciências sociais (veja, por exemplo, Diggle et al., 1994). A crescente popularidade destes modelos se deve à flexibilidade que eles oferecem para modelar a correlação entre e intra-unidades amostrais, freqüentemente presente em dados longitudinais (Laird e Ware, 1982) e dados agrupados (Henderson, 1984), pela capacidade de modelar dados balanceados e desbalanceados e pela existência de programas computacionais eficientes e confiáveis para ajustá-los (Wolfinger et al., 1991). Os MLM's têm como casos particulares o modelo linear clássico, o modelo de componentes de variância e também os modelos hierárquicos (multiníveis).

O modelo normal linear misto mais freqüentemente encontrado na literatura para respostas contínuas, proposto por Laird e Ware (1982), é expresso como

$$
\mathbf{Y}_{i}=\mathbf{x}_{i}^{\top} \boldsymbol{\beta}+\mathbf{Z}_{i} \mathbf{b}_{i}+\boldsymbol{\epsilon}_{i}, \quad i=1, \ldots, m
$$

onde $\mathbf{Y}_{i}$ é um vetor de respostas de dimensão $n_{i} \times 1$ dos $m$ indivíduos, $\mathbf{x}_{i}^{\top}$ de dimensão $n_{i} \times p$ é a matriz modelo correspondente aos efeitos fixos, $\boldsymbol{\beta}$ de dimensão $p \times 1$ é o vetor dos coeficientes da regressão da média populacional, chamado de efeitos fixos, $\mathbf{Z}_{i}$ de dimensão $n_{i} \times q$ é a matriz modelo correspondente ao vetor de efeitos aleatórios $\mathbf{b}_{i}$ de dimensão $q \times 1$ e $\boldsymbol{\epsilon}_{i}$ de dimensão $n_{i} \times 1$ é o vetor de erros aleatórios. Tipicamente assume-se que os efeitos aleatórios $\mathbf{b}_{i}$ e os componentes residuais $\boldsymbol{\epsilon}_{i}$ são independentes com $\mathbf{b}_{i} \stackrel{i i d}{\sim} N_{q}(\mathbf{0}, \mathbf{B})$, e $\boldsymbol{\epsilon}_{i} \stackrel{i n d}{\sim} N_{n_{i}}\left(\mathbf{0}, \boldsymbol{\psi}_{i}\right)$, para $i=1, \ldots, m$, onde $\mathbf{B}=\mathbf{B}(\boldsymbol{\alpha})$ e $\boldsymbol{\psi}_{i}=\psi_{i}(\gamma)$ são matrizes de dispersão, usualmente 
associadas com a variabilidade entre e intra-unidades amostrais, sendo dependentes de um número de parâmetros $\boldsymbol{\alpha}$ e $\boldsymbol{\gamma}$ desconhecidos, respectivamente.

Recentemente, Arellano-Valle et al. (2005)(veja também Lin e Lee, 2008) propuseram um modelo linear misto normal assimétrico baseado na distribuição normal assimétrica multivariada introduzida por Azzalini e Dalla-Valle (1996) e Azzalini e Capitanio (1999). O modelo é escrito como

$$
\begin{aligned}
& \mathbf{Y}_{i}= \mathbf{x}_{i}^{\top} \boldsymbol{\beta}+\mathbf{Z}_{i} \mathbf{b}_{i}+\boldsymbol{\epsilon}_{i}, \quad i=1, \ldots, m, \\
& \mathbf{b}_{i} \stackrel{i i d}{\sim} N A_{q}(\mathbf{0}, \mathbf{B}, \boldsymbol{\lambda}), \quad \boldsymbol{\epsilon}_{i} \stackrel{i n d}{\sim} N_{n_{i}}\left(\mathbf{0}, \boldsymbol{\psi}_{i}\right), \quad \mathbf{b}_{i} \perp \boldsymbol{\epsilon}_{i}, i=1, \ldots, m,
\end{aligned}
$$

onde $\boldsymbol{\lambda}=\left(\lambda_{1}, \ldots, \lambda_{q}\right)^{\top}$ é o vetor de assimetria de $Z$. Como apontado em Arellano-Valle et al. (2005), o modelo normal linear misto em (5.2) pode ser representado hierarquicamente como

$$
\begin{aligned}
\mathbf{Y}_{i} \mid \mathbf{b}_{i} & \stackrel{i n d}{\sim} N_{n_{i}}\left(\mathbf{x}_{i}^{\top} \boldsymbol{\beta}+\mathbf{Z}_{i} \mathbf{b}_{i}, \boldsymbol{\psi}_{i}\right) \\
\mathbf{b}_{i} & \stackrel{i i d}{\sim} N A_{q}(\mathbf{0}, \mathbf{B}, \boldsymbol{\lambda}), \quad i=1, \ldots, m .
\end{aligned}
$$

\subsection{Modelo Linear Misto de Misturas de Escala Nor- mal Assimétrico}

Como foi visto no Capítulo 4, a distribuição condicional de $U \mid Y$ para o modelo exponencial potência não é conhecida, bem como a forma explícita de $\kappa(u)$. Assim, neste capítulo não será tratada a estimação dos modelos de mistura envolvendo esta distribuição. Sabe-se que, para as demais distribuições da família (normal, t de Student generalizada, slash e normal contaminada), $\kappa(u)=1 / u$.

O modelo misto desenvolvido neste trabalho estende o modelo misto em (5.2) por considerar $b_{i}$ com distribuição na classe de misturas de escalas normais assimétricas, vista no Capítulo 4. Para tanto, faz-se necessário estender para o contexto multivariado as distribuições normal assimétrica, de mistura de escala normal e de mistura de escala normal assimétrica

Definição 5.1. Um vetor aleatório $m$-dimensional $\mathbf{Y}$ segue uma distribuição de mistura de escala normal multivariada com vetor de locação $\boldsymbol{\mu} \in \mathbb{R}_{n}$, matriz de dispersão $\boldsymbol{\Sigma}$ (matriz definida positiva $n \times n)$ e $H(u ; \boldsymbol{\tau})$ função distribuição de probabilidade da variável de mistura 
$U$, se sua função densidade de probabilidade (fdp) é dada por

$$
\begin{aligned}
f(\mathbf{y}) & =\int_{0}^{\infty} \phi_{n}\left(\boldsymbol{\mu}, u^{-1} \boldsymbol{\Sigma}\right) d H(u, \boldsymbol{\tau}) \\
& =|2 \pi \boldsymbol{\Sigma}|^{-1 / 2} \int_{0}^{\infty} u^{n / 2} \exp \left[-\frac{1}{2} u(\mathbf{y}-\boldsymbol{\mu})^{\top} \boldsymbol{\Sigma}^{-1}(\mathbf{y}-\boldsymbol{\mu})\right] d H(u, \boldsymbol{\tau}), \mathbf{y} \in \mathbb{R}^{n}
\end{aligned}
$$

com notação $\mathbf{Y} \sim M E N_{n}(\boldsymbol{\mu}, \boldsymbol{\Sigma} ; H)$.

Sendo $\mathbf{Y} \sim M E N_{n}(\boldsymbol{\mu}, \boldsymbol{\Sigma} ; H)$, admite-se a representação

$$
\mathbf{Y} \stackrel{d}{=} \boldsymbol{\mu}+U^{-1 / 2} \mathbf{Z}
$$

em que $\mathbf{Z} \sim N_{n}(\mathbf{0}, \boldsymbol{\Sigma}), U$ é uma variável aleatória positiva com distribuição $H$, independente de $\mathbf{Z}$. As distribuições de mistura de escala normal multivariadas são idênticas às distribuições vistas na Seção 4.3.1, $\operatorname{com} \boldsymbol{\mu}=\mu$ e $\boldsymbol{\Sigma}=\sigma^{2}$ (veja Salgado, 2006 ou Lange e Sinsheimer, 1993).

Definição 5.2. Um vetor aleatório n-dimensional $\mathbf{Y}$ segue uma distribuição normal assimétrica com vetor de locação $\boldsymbol{\mu} \in \mathbb{R}^{n}$, matriz de dispersão $\boldsymbol{\Sigma}$ (matriz semi definida positiva $n \times n)$ e vetor de assimetria $\boldsymbol{\lambda} \in \mathbb{R}^{n}$, se sua fdp é dada por

$$
f_{\mathbf{Y}}(\mathbf{y})=2 \phi_{n}(\mathbf{y} \mid \boldsymbol{\mu}, \boldsymbol{\Sigma}) \Phi_{1}\left(\boldsymbol{\lambda}^{\top} \boldsymbol{\Sigma}^{-1 / 2}(\mathbf{y}-\boldsymbol{\mu})\right), \quad \mathbf{y} \in \mathbb{R}^{n},
$$

com notação $\mathbf{Y} \sim N A_{n}(\boldsymbol{\mu}, \boldsymbol{\Sigma}, \boldsymbol{\lambda})$ e $\mathbf{Y} \sim N A_{n}(\boldsymbol{\lambda})$ quando $\boldsymbol{\mu}=0$ e $\boldsymbol{\Sigma}=\mathbb{I}_{n}$, a matriz identidade de ordem $n$.

Definição 5.3. Um vetor aleatório $n$-dimensional $\mathbf{Y}$ segue uma distribuição de mistura de escala normal assimétrica multivariada com vetor de locação $\boldsymbol{\mu} \in \mathbb{R}_{n}$, matriz de dispersão $\boldsymbol{\Sigma}$ (matriz definida positiva $n \times n)$ e $H(u ; \boldsymbol{\tau})$ função distribuição de probabilidade da variável de mistura $U$, se sua fdp é dada por

$$
f(\mathbf{y})=2 \int_{0}^{\infty} \phi_{n}\left(\mathbf{y}, \boldsymbol{\mu}, u^{-1} \boldsymbol{\Sigma}\right) \Phi_{1}\left(\boldsymbol{\lambda}^{\top} \boldsymbol{\Sigma}^{-1 / 2}(\mathbf{y}-\boldsymbol{\mu})\right) d H(u, \boldsymbol{\tau}), \mathbf{y} \in \mathbb{R}^{n}
$$

com notação $\mathbf{Y} \sim M E N A_{n}(\boldsymbol{\mu}, \boldsymbol{\Sigma}, \boldsymbol{\lambda} ; H)$.

Observação: Condicionado em $U$, tem-se que

$$
\begin{aligned}
f_{\mathbf{Y} \mid U=u}(\mathbf{y}) & =2 \phi_{n}\left(\mathbf{y}, \boldsymbol{\mu}, u^{-1} \boldsymbol{\Sigma}\right) \Phi_{1}\left(\boldsymbol{\lambda}^{\top} \boldsymbol{\Sigma}^{-1 / 2}(\mathbf{y}-\boldsymbol{\mu})\right) \\
& =2 \phi_{n}\left(\mathbf{y}, \boldsymbol{\mu}, u^{-1} \boldsymbol{\Sigma}\right) \Phi_{1}\left(u^{-1 / 2} \boldsymbol{\lambda}^{\top}\left[\left(u^{-1} \boldsymbol{\Sigma}\right)^{-1 / 2}\right](\mathbf{y}-\boldsymbol{\mu})\right) .
\end{aligned}
$$

Ferreira, C. S. 
Portanto, $\mathbf{Y} \mid U=u \sim N A_{n}\left(\boldsymbol{\mu}, u^{-1} \boldsymbol{\Sigma}, u^{-1 / 2} \boldsymbol{\lambda}\right)$.

O Modelo Linear Misto de Misturas de Escala Normal Assimétrico (MLMMENA) apresentado neste trabalho é dado por

$$
\begin{aligned}
& \mathbf{Y}_{i}=\mathbf{x}_{i}^{\top} \boldsymbol{\beta}+\mathbf{Z}_{i} b_{i}+\boldsymbol{\epsilon}_{i} \\
& \left(\begin{array}{c}
b_{i} \\
\boldsymbol{\epsilon}_{i}
\end{array}\right) \sim M E N A_{n_{i}+1}\left(\left(\begin{array}{l}
0 \\
\mathbf{0}
\end{array}\right),\left(\begin{array}{cc}
\sigma_{b}^{2} & \mathbf{0} \\
\mathbf{0} & \sigma_{e}^{2} \mathbb{I}_{n_{i}}
\end{array}\right),\left(\begin{array}{l}
\lambda \\
\mathbf{0}
\end{array}\right) ; H\right), \quad i=1, \ldots, m .
\end{aligned}
$$

Assim, o erro tem distribuição simétrica e o efeito aleatório tem distribuição assimétrica. Embora não seja possível encontrar uma distribuição marginal para $\mathbf{Y}_{i}$, uma representação estocástica é alcançada, o que possibilita um procedimento de estimação dos parâmetros do modelo através do algoritmo EM. Embora $b_{i}$ e $\boldsymbol{\epsilon}_{i}$ não sejam independentes, devido à dependência mútua em $U_{i}$, pode ser provado que os mesmos são não correlacionados. Para mostrar esta relação, será utilizado um resultado provado em Lachos (2004).

Proposição 5.1. Sejam $\mathbf{Y} \sim N A_{n}(\boldsymbol{\mu}, \boldsymbol{\Sigma}, \boldsymbol{\lambda}), \mathbf{C}(n \times k)$ uma matriz de posto completo $k$, $k \leq n$ e $h$ um inteiro positivo. Adicionalmente, suponha que $\boldsymbol{\lambda}$ e $\mathbf{C}$ sejam particionados em $\boldsymbol{\lambda}=\left(\boldsymbol{\lambda}_{1}^{\top}, \ldots, \boldsymbol{\lambda}_{h}^{\top}\right)^{\top}$ e $\mathbf{C}=\left(\mathbf{C}_{1}, \ldots, \mathbf{C}_{h}\right)$, onde $\mathbf{C}_{i}$ de dimensão $n \times r_{i}, i=1, \ldots, h$, com $\sum_{i=1}^{h} r_{i}=k$. Se, simultaneamente,

(i) $\mathbf{C}_{i}^{\top} \mathbf{\Sigma} \mathbf{C}_{j}=\mathbf{0}, i \neq j, e$

(ii) $\boldsymbol{\delta}^{\top} \boldsymbol{\Sigma}^{1 / 2} \mathbf{C}_{i} \neq \mathbf{0}$, para no máximo um $i$,

com $\boldsymbol{\delta}=\frac{\boldsymbol{\lambda}}{\sqrt{1+\boldsymbol{\lambda}^{\top} \boldsymbol{\lambda}}}$, então $\mathbf{C}_{i}^{\top} \mathbf{Y}$ é independente de $\mathbf{C}_{j}^{\top} \mathbf{Y}, i \neq j, i, j=1, \ldots, h$.

Condicionando em $U$, tem-se que a distribuição conjunta de $b_{i}$ e $\boldsymbol{\epsilon}_{i}$ é dada por

$$
\left(\begin{array}{c}
b_{i} \\
\boldsymbol{\epsilon}_{i}
\end{array}\right) \mid U_{i}=u_{i} \sim N A_{n_{i}+1}\left(\left(\begin{array}{c}
0 \\
\mathbf{0}
\end{array}\right), u^{-1}\left(\begin{array}{cc}
\sigma_{b}^{2} & \mathbf{0} \\
\mathbf{0} & \sigma_{e}^{2} \mathbb{I}_{n_{i}}
\end{array}\right), u^{-1 / 2}\left(\begin{array}{c}
\lambda \\
\mathbf{0}
\end{array}\right) ; H\right), \quad i=1, \ldots, m .
$$

Considerando a distribuição condicional em (5.7), $h=2, \mathbf{C}_{1}=(1 \mathbf{0})\left(1 \times\left(n_{i}+1\right)\right)$ e $\mathbf{C}_{2}=\left(\mathbf{0} \mathbb{I}_{n_{i}}\right)\left(n_{i} \times\left(n_{i}+1\right)\right)$, tem-se que $\mathbf{C}_{1}^{\top} \mathbf{Y}=b_{i}$ e $\mathbf{C}_{2}^{\top} \mathbf{Y}=\boldsymbol{\epsilon}_{i}$, as hipóteses da Proposição 5.1 são satisfeitas. Portanto, $b_{i} \mid U_{i}$ e $\boldsymbol{\epsilon}_{i}$ são condicionalmente independentes, dado $U_{i}$ e então $E\left[\boldsymbol{\epsilon}_{i}\right]=\mathbf{0}$.

A seguir, são calculadas as distribuições marginais de $b_{i}$ e $\boldsymbol{\epsilon}_{i}$. Para tanto, será utilizado 
um resultado encontrado em Lachos (2004), dado pela seguinte proposição.

Proposição 5.2. Sejam $\mathbf{Y} \sim N A_{n}(\boldsymbol{\mu}, \boldsymbol{\Sigma}, \boldsymbol{\lambda})$ e $\mathbf{C}$ uma matriz $n \times k$ de posto completo. Então

$$
\mathbf{C}^{\top} \mathbf{Y} \sim N A_{k}\left(\mathbf{C}^{\top} \boldsymbol{\mu}, \mathbf{C}^{\top} \boldsymbol{\Sigma} \mathbf{C}, \boldsymbol{\lambda}^{*}\right)
$$

onde $\boldsymbol{\lambda}^{*}=\boldsymbol{\delta}^{*} /\left(1-\boldsymbol{\delta}^{* \top} \boldsymbol{\delta}^{*}\right)^{1 / 2}, \operatorname{com} \boldsymbol{\delta}^{*}=\left(\mathbf{C}^{\top} \boldsymbol{\Sigma} \mathbf{C}\right)^{-1 / 2} \mathbf{C}^{\top} \boldsymbol{\Sigma}^{1 / 2} \boldsymbol{\delta}$.

Considerando a distribuição condicional em (5.7), $\boldsymbol{\delta}=\frac{u_{i}^{-1 / 2}}{\sqrt{1+u_{i}^{-1} \lambda^{2}}}\left(\begin{array}{l}\lambda \\ \mathbf{0}\end{array}\right)$. Fazendo $\mathbf{C}=$ (1 0 ) $\left(1 \times\left(n_{i}+1\right)\right)$, tem-se que $\mathbf{C}^{\top} \mathbf{Y}=b_{i}, \mathbf{C}^{\top} \boldsymbol{\Sigma} \mathbf{C}=\sigma_{b}^{2} / u_{i}, \mathbf{C}^{\top} \boldsymbol{\Sigma}^{1 / 2} \boldsymbol{\delta}=\frac{\lambda \sigma_{b}}{u_{i} \sqrt{1+u_{i}^{-1} \lambda^{2}}} \mathrm{e}$ $\delta^{*}=\frac{\lambda}{\sqrt{u_{i}+\lambda^{2}}}, \lambda^{*}=\frac{\lambda}{\sqrt{u_{i}}}$. Portanto,

$$
b_{i} \mid U_{i}=u_{i} \sim N A\left(0, \frac{\sigma_{b}^{2}}{u_{i}}, \frac{\lambda}{\sqrt{u_{i}}}\right) .
$$

Logo,

$$
b_{i} \sim \operatorname{MENA}\left(0, \sigma_{b}^{2}, \lambda ; H\right)
$$

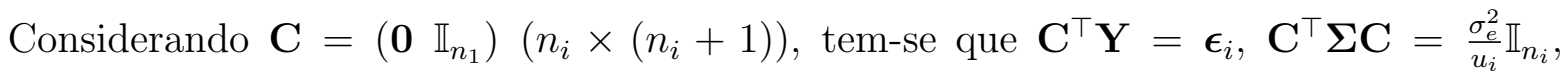
$\mathbf{C}^{\top} \boldsymbol{\Sigma}^{1 / 2} \boldsymbol{\delta}=\mathbf{0}, \delta^{*}=\mathbf{0}, \lambda^{*}=\mathbf{0}$. Portanto,

$$
\boldsymbol{\epsilon}_{i} \mid U_{i}=u_{i} \sim N_{n_{i}}\left(\mathbf{0}, \frac{\sigma_{e}^{2}}{u_{i}} \mathbb{I}_{n_{i}}\right)
$$

Logo,

$$
\boldsymbol{\epsilon} \sim M E N_{n_{i}}\left(\mathbf{0}, \sigma_{e}^{2} \mathbb{I}_{n_{i}} ; H\right)
$$

Portanto, $E\left[\boldsymbol{\epsilon}_{i}\right]=\mathbf{0}$ e

$$
\operatorname{Cov}\left(b_{i}, \boldsymbol{\epsilon}_{i}\right)=E\left[b_{i} \boldsymbol{\epsilon}_{i}\right]-E\left[b_{i}\right] E\left[\boldsymbol{\epsilon}_{i}\right]=E_{U_{i}}\left[E\left[b_{i} \mid U_{i}\right] E\left[\boldsymbol{\epsilon}_{i} \mid U_{i}\right]\right]=\mathbf{0}
$$

Assim, usando (5.9) e (5.11), o Modelo Linear Misto de Misturas de Escala Normal Assimétrico (MLMMENA) pode ser representado como

$$
\begin{aligned}
\mathbf{Y}_{i} & =\mathbf{x}_{i}^{\top} \boldsymbol{\beta}+\mathbf{Z}_{i} b_{i}+\boldsymbol{\epsilon}_{i}, \\
b_{i} & \stackrel{i i d}{\sim} \operatorname{MENA}\left(0, \sigma_{b}^{2}, \lambda ; H\right), \\
\boldsymbol{\epsilon}_{i} & \stackrel{i n d}{\sim} \operatorname{MEN}_{n_{i}}\left(\mathbf{0}, \sigma_{e}^{2} \mathbb{I}_{n_{i}} ; H\right), \quad i=1, \ldots, m .
\end{aligned}
$$

Ferreira, C. S. 
Utilizando a distribuição condicional de $b_{i} \mid U_{i}$ em (5.8), o modelo pode ser representado hierarquicamente da seguinte forma:

$$
\begin{aligned}
\mathbf{Y}_{i} \mid b_{i}, u_{i} & \stackrel{\text { ind }}{\sim} N_{n_{i}}\left(\mathbf{x}_{i}^{\top} \boldsymbol{\beta}+\mathbf{Z}_{i} b_{i}, u^{-1} \sigma_{e}^{2} \mathbb{I}_{n_{i}}\right), \\
b_{i} \mid u_{i} & \stackrel{\text { ind }}{\sim} N A\left(0, u_{i}^{-1} \sigma_{b}^{2}, u_{i}^{-1 / 2} \lambda\right) \mathrm{e} \\
u_{i} & \stackrel{\text { iid }}{\sim} H\left(u_{i} ; \boldsymbol{\tau}\right) .
\end{aligned}
$$

Lee e Nelder (2004) fazem uma discussão entre modelos condicionais e marginais em modelos com efeitos aleatórios, discutindo as vantagens do condicional em relação ao marginal e consideram o primeiro como fundamental, do qual predições marginais podem ser feitas.

Se $Z \sim M E N\left(0, \sigma^{2} ; H\right)$, então $E[Z]=0$. Do Capítulo 4 , sendo $b_{i} \sim \operatorname{MENA}\left(0, \sigma_{b}^{2}, \lambda ; H\right)$, então $E\left[b_{i}\right]=c \sigma_{b} \lambda E_{U_{i}}\left[\frac{U^{-1}}{\sqrt{1+U^{-1} \lambda^{2}}}\right]$, com $c=\sqrt{\frac{2}{\pi}}$. Portanto,

$$
E\left[\mathbf{Y}_{i}\right]=\mathbf{x}_{i} \boldsymbol{\beta}+c \sigma_{b} \lambda E_{U_{i}}\left[\frac{U^{-1}}{\sqrt{1+U^{-1} \lambda^{2}}}\right] \mathbf{Z}_{i} .
$$

A função correspondente (5.13) é dada por

$$
f\left(\mathbf{y}_{i}\right)=2 \int_{-\infty}^{+\infty} \int_{0}^{+\infty} \phi_{n_{i}}\left(\mathbf{y}_{i} \mid \mathbf{x}_{i}^{\top} \boldsymbol{\beta}+b_{i} \mathbf{Z}_{i}, u_{i}^{-1} \sigma_{e}^{2} \mathbb{I}_{n_{i}}\right) \phi\left(b_{i} \mid 0, u_{i}^{-1} \sigma_{b}^{2}\right) \Phi\left(\frac{\lambda b_{i}}{\sigma_{b}}\right) h\left(u_{i} ; \boldsymbol{\tau}\right) d u_{i} d b_{i} .
$$

Se $\lambda=0$ e $U=1$, o modelo (5.12) se torna o modelo misto clássico de efeitos aleatórios. Se $U=1$, o mesmo se torna o modelo de regressão misto normal assimétrico introduzido por Arellano-Valle, Bolfarine e Lachos (2005), porém com $b_{i}$ escalar. Se $b_{i}=0$, tem-se o modelo de misturas de escalas normal assimétrico, visto no Capítulo 4. Zhou e He (2007) apresentam um modelo misto assimétrico considerando ambos efeito aleatório e o erro seguindo distribuição t-assimétrica (Azzalini e Capitanio, 2003), sendo $b_{i}$ unidimensional. O modelo misto apresentado neste trabalho, além de generalizar a classe de modelos mistos normais assimétricos, possibilita um processo de estimação através do algoritmo EM, com representação analítica no passo M, além de obtermos uma aproximação para a matriz de informação de Fisher observada. 


\subsection{Algoritmo EM}

O modelo misto em (5.13) pode ser reescrito como

$$
\begin{aligned}
\mathbf{Y}_{i} \mid b_{i}, u_{i}, t_{i} & \stackrel{\text { ind }}{\sim} N_{n_{i}}\left(\mathbf{x}_{i}^{\top} \boldsymbol{\beta}+\mathbf{Z}_{i} b_{i}, u^{-1} \sigma_{e}^{2} \mathbb{I}_{n_{i}}\right), \\
b_{i} \mid u_{i}, t_{i} & \stackrel{\text { ind }}{\sim} N\left(\frac{\sigma_{b} \lambda}{u_{i} \sqrt{1+\lambda^{2} / u_{i}}} t_{i}, \frac{\sigma_{b}^{2}}{u_{i}+\lambda^{2}}\right) \\
U_{i} & \stackrel{\text { iid }}{\sim} H\left(u_{i} ; \boldsymbol{\tau}\right) \mathrm{e} \\
T_{i} & \stackrel{\text { iid }}{\sim} N T(0,1), i=1, \ldots, m .
\end{aligned}
$$

e assim a função densidade (5.15) pode ser escrita como

$$
\begin{aligned}
f\left(\mathbf{y}_{i}\right) & =2 \int_{-\infty}^{+\infty} \int_{0}^{+\infty} \int_{0}^{+\infty} \phi_{n_{i}}\left(\mathbf{y}_{i} \mid \mathbf{x}_{i}^{\top} \boldsymbol{\beta}+b_{i} \mathbf{Z}_{i}, \sigma_{e}^{2} / u_{i} \mathbb{I}_{n_{i}}\right) \phi\left(b_{i} \mid 0, \sigma_{b}^{2} / u_{i}\right) \\
& \times \phi\left(t_{i} \mid \lambda b_{i}, \sigma_{b}^{2}\right) \mathbf{I}\left(t_{i}>0\right) h\left(u_{i} ; \boldsymbol{\tau}\right) d t_{i} d u_{i} d b_{i} .
\end{aligned}
$$

Sejam $\mathbf{y}=\left(\mathbf{y}_{1}^{\top}, \ldots, \mathbf{y}_{m}^{\top}\right)^{\top}, \mathbf{b}=\left(b_{1}, \ldots, b_{m}\right)^{\top} \mathbf{u}=\left(u_{1}, \ldots, u_{m}\right)^{\top}$ e $\mathbf{t}=\left(t_{1}, \ldots, t_{m}\right)^{\top}$ e tratando $\mathbf{u}, \mathbf{b}$ e t como dados faltantes, segue que a função log-verossimilhança completa associada com $\mathbf{y}_{c}=\left(\mathbf{y}^{\top}, \mathbf{u}^{\top}, \mathbf{b}^{\top}, \mathbf{t}^{\top}\right)^{\top}$ é dada por

$$
\begin{aligned}
\ell_{c}\left(\boldsymbol{\theta} \mid \mathbf{y}_{c}\right) \propto & -\frac{N}{2} \log \sigma_{e}^{2}-\frac{1}{2 \sigma_{e}^{2}} \sum_{i=1}^{m} u_{i}\left(\mathbf{y}_{i}-\mathbf{x}_{i}^{\top} \boldsymbol{\beta}-b_{i} \mathbf{Z}_{i}\right)^{\top}\left(\mathbf{y}_{i}-\mathbf{x}_{i}^{\top} \boldsymbol{\beta}-b_{i} \mathbf{Z}_{i}\right) \\
& -m \log \sigma_{b}^{2}-\frac{1}{2 \sigma_{b}^{2}} \sum_{i=1}^{m} u_{i} b_{i}^{2}-\frac{1}{2 \sigma_{b}^{2}} \sum_{i=1}^{m}\left(t_{i}^{2}-2 \lambda t_{i} b_{i}+\lambda^{2} b_{i}^{2}\right),
\end{aligned}
$$

onde $N=\sum_{i=1}^{m} n_{i}$

Denote por $\boldsymbol{\theta}^{(k)}=\left(\boldsymbol{\beta}^{(k)^{\top}}, \sigma_{e}{ }^{2(k)}, \sigma_{b}{ }^{2(k)}, \lambda^{(k)}\right)^{\top}$ a estimativa de $\boldsymbol{\theta}$ na $k$-ésima iteração. Segue que a esperança com respeito a $\mathbf{t}, \mathbf{u}, \mathbf{b}$, condicionada em $\mathbf{y}$, da função log-verossimilhança completa (Passo E), tem a forma

$$
\begin{aligned}
& Q\left(\boldsymbol{\theta} \mid \widehat{\boldsymbol{\theta}}^{k}\right)=\mathrm{E}\left[\ell_{c}\left(\boldsymbol{\theta} \mid \mathbf{y}_{c}\right) \mid \mathbf{y}, \widehat{\boldsymbol{\theta}}^{(k)}\right] \\
& =-\frac{1}{2 \sigma_{e}{ }^{(k)}} \sum_{i=1}^{m}\left(\widehat{u}_{i}^{(k)}\left(\mathbf{y}_{i}-\mathbf{x}_{i}^{\top} \boldsymbol{\beta}^{(k)}\right)^{\top}\left(\mathbf{y}_{i}-\mathbf{x}_{i}^{\top} \boldsymbol{\beta}^{(k)}\right)-2 \widehat{u b}{ }_{i}^{(k)} \mathbf{Z}_{i}^{\top}\left(\mathbf{y}_{i}-\mathbf{x}_{i}^{\top} \boldsymbol{\beta}^{(k)}\right)+{\widehat{u b^{2}}}_{i}^{(k)} \mathbf{Z}_{i}^{\top} \mathbf{Z}_{i}\right) \\
& \quad-\frac{N}{2} \log \sigma_{e}^{2^{(k)}}-m \log \sigma_{b}^{2^{(k)}}-\frac{1}{2{\sigma_{b}^{2}}^{(k)}} \sum_{i=1}^{m}\left({\widehat{u b b^{2}}}_{i}^{(k)}+\widehat{t t}^{2}{ }_{i}^{(k)}-2 \lambda^{(k)} \widehat{t b}_{i}^{(k)}+\lambda^{(k)^{2}} \widehat{b}_{i}^{2(k)}\right),
\end{aligned}
$$


onde ${\widehat{t^{d} u^{e} b^{f}}}_{i}^{(k)}=E\left[T_{i}^{d} U_{i}^{e} b_{i}^{f} \mid \mathbf{y}_{i}, \boldsymbol{\theta}^{(k)}\right], d, e, f>0$.

O Passo M maximiza $Q\left(\boldsymbol{\theta} \mid \widehat{\boldsymbol{\theta}}^{(k)}\right)$ com respeito a $\boldsymbol{\theta}$, obtendo uma nova estimativa $\widehat{\boldsymbol{\theta}}^{(k+1)}$, como descrito abaixo.

Passo E: Dado $\boldsymbol{\theta}=\widehat{\boldsymbol{\theta}}^{(k)}$, calcule para $i=1, \ldots, m, \widehat{u}_{i}^{(k)}, \widehat{u b}_{i}^{(k)},{\widehat{u b^{2}}}^{(k)},{\widehat{b^{2}}}_{i}^{(k)}, \widehat{t b}_{i}^{(k)}$ e ${\widehat{t^{2}}}^{(k)}$ usando (5.24)-(5.29).

Passo M: Atualize $\widehat{\boldsymbol{\theta}}^{(k+1)}$ maximizando $Q\left(\boldsymbol{\theta} \mid \widehat{\boldsymbol{\theta}}^{(k)}\right)$ sobre $\boldsymbol{\theta}$, que leva às seguintes expressões:

$$
\begin{aligned}
\widehat{\boldsymbol{\beta}}^{(k+1)} & =\left(\mathbf{X}^{\top} \mathbf{D}\left(\mathbf{U}^{*^{(k)}}\right) \mathbf{X}\right)^{-1} \mathbf{X}^{\top}\left(\mathbf{D}\left(\mathbf{U}^{*(k)}\right) \mathbf{Y}-\mathbf{D}\left(\mathbf{U b}^{*(k)}\right) \mathbf{Z}\right) \\
\widehat{\lambda}^{(k+1)} & =\frac{\sum_{i=1}^{m} \widehat{t b}_{i}^{(k)}}{\sum_{i=1}^{m}{\widehat{b^{2}}}^{(k)}} \\
{\widehat{\sigma_{e}^{2}}}^{(k+1)} & =\frac{1}{N} \sum_{i=1}^{m}\left(\widehat{u}_{i}^{(k)}\left(\mathbf{y}_{i}-\mathbf{x}_{i}^{\top} \boldsymbol{\beta}^{(k)}\right)^{\top}\left(\mathbf{y}_{i}-\mathbf{x}_{i}^{\top} \boldsymbol{\beta}^{(k)}\right)-2 \widehat{u b}_{i}^{(k)} \mathbf{Z}_{i}^{\top}\left(\mathbf{y}_{i}-\mathbf{x}_{i}^{\top} \boldsymbol{\beta}^{(k)}\right)+{\widehat{u b^{2}}}_{i}^{(k)} \mathbf{Z}_{i}^{\top} \mathbf{Z}_{i}\right) \mathrm{e} \\
{\widehat{\sigma_{b}^{2}}}^{(k+1)} & =\frac{1}{2 m} \sum_{i=1}^{m}\left({\widehat{u b^{2}}}^{(k)}+\widehat{t}^{2}{ }_{i}^{(k)}-2 \lambda^{(k)} \widehat{t b}_{i}^{(k)}+\lambda^{(k)^{2}}{\widehat{b^{2}}}^{(k)}{ }^{(k)}\right)
\end{aligned}
$$

onde $\mathbf{X}=\left(\mathbf{x}_{1}^{\top}, \ldots, \mathbf{x}_{m}^{\top}\right)^{\top}$ de dimensão $N \times p, \mathbf{Z}=\left(\mathbf{Z}_{1}^{\top}, \ldots, \mathbf{Z}_{m}^{\top}\right)^{\top}$ de dimensão $N \times 1$, $\widehat{\mathbf{U}}^{*(k)}=\left[\widehat{u}_{1}^{(k)} \mathbf{1}_{n_{1}}^{\top}, \ldots, \widehat{u}_{m}^{(k)} \mathbf{1}_{n_{m}}^{\top}\right]^{\top}, \widehat{\mathbf{U b}}^{*(k)}=\left[\widehat{u b}_{1}^{(k)} \mathbf{1}_{n_{1}}^{\top}, \ldots, \widehat{u b}_{m}^{(k)} \mathbf{1}_{n_{m}}^{\top}\right]^{\top}$ e $\mathbf{1}_{k}$ é um vetor-coluna $k$-dimensional de 1 's, $k>0$.

\subsubsection{Distribuições condicionais para o algoritmo EM}

Para o cálculo das estimativas das variáveis latentes, presentes no algoritmo EM, serão feitos alguns passos a fim de se alcançar uma solução, se não-analítica, pelo menos em função de valores esperados em relação à distribuição de $U$. Para tanto, sabe-se que

$$
f\left(U_{i}, b_{i}, t_{i} \mid \mathbf{y}_{i}, \boldsymbol{\theta}\right)=f\left(b_{i} \mid \mathbf{y}_{i}, U_{i}, t_{i}, \boldsymbol{\theta}\right) f\left(t_{i} \mid \mathbf{y}_{i}, U_{i}, \boldsymbol{\theta}\right) f\left(U_{i} \mid \mathbf{y}_{i}, \boldsymbol{\theta}\right)
$$

Assim, por exemplo,

$$
\widehat{u b}_{i}=E_{U_{i}}\left[U_{i} E_{t_{i}}\left[E_{b_{i}}\left[b_{i} \mid \mathbf{y}_{i}, U_{i}, t_{i}\right] \mid \mathbf{y}_{i}, U_{i}\right] \mid \mathbf{y}_{i}, \boldsymbol{\theta}\right]
$$

Da equação (5.17) tem-se que

$$
f\left(\mathbf{y}_{i} \mid \boldsymbol{\theta}\right)=\int_{-\infty}^{+\infty} \int_{0}^{+\infty} \int_{0}^{+\infty} f\left(\mathbf{y}_{i} \mid b_{i}, U_{i}, \boldsymbol{\theta}\right) f\left(b_{i} \mid U_{i}, \boldsymbol{\theta}\right) f\left(t_{i} \mid b_{i}, \boldsymbol{\theta}\right) f\left(U_{i}, \boldsymbol{\theta}\right) d t_{i} d U_{i} d b_{i}
$$


Do Lema 2 de Arellano-Valle et al. (2005), sendo $k(u)=1 / u$, tem-se que

$$
\begin{aligned}
& f\left(\mathbf{y}_{i} \mid b_{i}, U_{i}, \boldsymbol{\theta}\right) f\left(b_{i} \mid U_{i}, \boldsymbol{\theta}\right)=\phi_{n_{i}}\left(\mathbf{y}_{i} \mid \mathbf{x}_{i}^{\top} \boldsymbol{\beta}+b_{i} \mathbf{Z}_{i}, \sigma_{e}^{2} / u_{i} \mathbb{I}_{n_{i}}\right) \phi\left(b_{i} \mid 0, \sigma_{b}^{2} / u_{i}\right) \\
& =\phi_{n_{i}}\left(\mathbf{y}_{i} \mid \mathbf{x}_{i}^{\top} \boldsymbol{\beta}, U_{i}^{-1}\left[\sigma_{e}^{2} \mathbb{I}_{n_{i}}+\sigma_{b}^{2} \mathbf{Z}_{i} \mathbf{Z}_{i}^{\top}\right]\right) \phi\left(b_{i} \mid \Lambda_{i} \sigma_{e}^{-2} U_{i} \mathbf{Z}_{i}^{\top}\left(\mathbf{y}_{i}-\mathbf{x}_{i}^{\top} \boldsymbol{\beta}\right), \Lambda_{i}\right)=f\left(\mathbf{y}_{i} \mid U_{i}, \boldsymbol{\theta}\right) f\left(b_{i} \mid \mathbf{y}_{i}, U_{i}, \boldsymbol{\theta}\right),
\end{aligned}
$$

onde $\Lambda_{i}=U_{i}^{-1}\left(\sigma_{b}^{-2}+\sigma_{e}^{-2} \mathbf{Z}_{i}^{\top} \mathbf{Z}_{i}\right)^{-1}$. Assim, a densidade conjunta é expressa como $f\left(\mathbf{y}_{i}, U_{i}, b_{i}, t_{i} \mid \boldsymbol{\theta}\right)=f\left(\mathbf{y}_{i} \mid U_{i}, \boldsymbol{\theta}\right) f\left(b_{i} \mid \mathbf{y}_{i}, U_{i}, \boldsymbol{\theta}\right) f\left(t_{i} \mid b_{i}, \boldsymbol{\theta}\right) f\left(U_{i}, \boldsymbol{\theta}\right)$. Usando novamente o mesmo Lema, tem-se que

$$
\begin{aligned}
f\left(b_{i} \mid \mathbf{y}_{i}, U_{i}, \boldsymbol{\theta}\right) f\left(t_{i} \mid b_{i}, \boldsymbol{\theta}\right) & =\phi\left(b_{i} \mid \Lambda_{i}^{*} \sigma_{e}^{-2} \mathbf{Z}_{i}^{\top}\left(\mathbf{y}_{i}-\mathbf{x}_{i}^{\top} \boldsymbol{\beta}\right), \Lambda_{i}\right) \phi\left(t_{i} \mid \lambda b_{i}, \sigma_{b}^{2}\right) \mathbf{I}\left(t_{i}>0\right) \\
& =\phi\left(b_{i} \mid \eta_{i}+\lambda \sigma_{b}^{-2} \delta_{i}\left(t_{i}-\lambda \eta_{i}\right), \delta_{i}\right) \phi\left(t_{i} \mid \lambda \eta_{i}, \sigma_{b}^{2}+\lambda^{2} \Lambda_{i}\right) \mathbf{I}\left(t_{i}>0\right) \\
& =f\left(b_{i} \mid \mathbf{y}_{i}, U_{i}, t_{i}, \boldsymbol{\theta}\right) f\left(t_{i} \mid \mathbf{y}_{i}, U_{i}, \boldsymbol{\theta}\right),
\end{aligned}
$$

onde $\eta_{i}=\Lambda_{i}^{*} \sigma_{e}^{-2} \mathbf{Z}_{i}^{\top}\left(\mathbf{y}_{i}-\mathbf{x}_{i}^{\top} \boldsymbol{\beta}\right), \Lambda_{i}^{*}=\left(\sigma_{b}^{-2}+\sigma_{e}^{-2} \mathbf{Z}_{i}^{\top} \mathbf{Z}_{i}\right)^{-1}$ e $\delta_{i}=\left(\Lambda_{i}^{-1}+\lambda^{2} \sigma_{b}^{-2}\right)^{-1}$. Portanto, chega-se à relação

$$
\begin{aligned}
f\left(U_{i}, b_{i}, t_{i} \mid \mathbf{y}_{i}, \boldsymbol{\theta}\right) & =\phi_{n_{i}}\left(\mathbf{y}_{i} \mid \mathbf{x}_{i}^{\top} \boldsymbol{\beta}, U_{i}^{-1}\left[\sigma_{e}^{2} \mathbb{I}_{n_{i}}+\sigma_{b}^{2} \mathbf{Z}_{i} \mathbf{Z}_{i}^{\top}\right]\right) \\
& \times \phi\left(b_{i} \mid \eta_{i}+\lambda \sigma_{b}^{-2} \delta_{i}\left(t_{i}-\lambda \eta_{i}\right), \delta_{i}\right) \phi\left(t_{i} \mid \lambda \eta_{i}, \sigma_{b}^{2}+\lambda^{2} \Lambda_{i}\right) \mathbf{I}\left(t_{i}>0\right) .
\end{aligned}
$$

Dado que $t_{i} \mid \mathbf{y}_{i}, U_{i}, \boldsymbol{\theta} \sim N T\left(\lambda \eta_{i}, \sigma_{b}^{2}+\lambda^{2} \Lambda_{i}\right)$, tem-se que

$$
\begin{gathered}
E\left[T_{i} \mid \mathbf{y}_{i}, U_{i}, \boldsymbol{\theta}\right]=\lambda \eta_{i}+\sqrt{\sigma_{b}^{2}+\lambda^{2} \Lambda_{i}} W_{\Phi}\left(\frac{\lambda \eta_{i}}{\sqrt{\sigma_{b}^{2}+\lambda^{2} \Lambda_{i}}}\right) \mathrm{e} \\
E\left[T_{i}^{2} \mid \mathbf{y}_{i}, U_{i}, \boldsymbol{\theta}\right]=\lambda^{2} \eta_{i}^{2}+\sigma_{b}^{2}+\lambda^{2} \Lambda_{i}+\lambda \eta_{i} \sqrt{\sigma_{b}^{2}+\lambda^{2} \Lambda_{i}} W_{\Phi}\left(\frac{\lambda \eta_{i}}{\sqrt{\sigma_{b}^{2}+\lambda^{2} \Lambda_{i}}}\right) .
\end{gathered}
$$

De acordo com (5.21), tem-se que $b_{i} \mid \mathbf{y}_{i}, U_{i}, t_{i}, \boldsymbol{\theta} \sim N\left(\eta_{i}+\frac{\lambda}{\sigma_{2}^{b}} \delta_{i}\left(t_{i}-\lambda \eta_{i}\right), \delta_{i}\right)$ e então as quantidades necessárias para o algoritmo EM são dadas nas equações a seguir:

$$
\widehat{u b}_{i}=\eta_{i} \widehat{U}_{i}+\frac{\lambda}{\sigma_{b}^{2}} E_{U_{i}}\left[U_{i} \delta_{i} \sqrt{\sigma_{b}^{2}+\lambda^{2} \Lambda_{i}} W_{\Phi}\left(\frac{\lambda \eta_{i}}{\sqrt{\sigma_{b}^{2}+\lambda^{2} \Lambda_{i}}}\right) \mid \mathbf{y}_{i}, \boldsymbol{\theta}\right]
$$




$$
\begin{aligned}
& {\widehat{u b^{2}}}_{i}=\eta_{i}^{2} \widehat{U}_{i}+\frac{\lambda^{2}}{\sigma_{b}^{2}} E_{U_{i}}\left(U_{i} \delta_{i}^{2} \mid \mathbf{y}_{i}, \boldsymbol{\theta}\right)+\frac{2 \lambda \eta_{i}}{\sigma_{b}^{2}} E_{U_{i}}\left[U_{i} \delta_{i} \sqrt{\sigma_{b}^{2}+\lambda^{2} \Lambda_{i}} W_{\Phi}\left(\frac{\lambda \eta_{i}}{\sqrt{\sigma_{b}^{2}+\lambda^{2} \Lambda_{i}}}\right) \mid \mathbf{y}_{i}, \boldsymbol{\theta}\right] \\
& +E_{U_{i}}\left[U_{i} \delta_{i} \mid \mathbf{y}_{i}, \boldsymbol{\theta}\right]+\frac{\lambda^{4} \Lambda_{i}^{*}}{\sigma_{b}^{4}} E_{U_{i}}\left(\delta_{i}^{2} \mid \mathbf{y}_{i}, \boldsymbol{\theta}\right) \\
& -\frac{\lambda^{3} \eta_{i}}{\sigma_{b}^{4}} E_{U_{i}}\left[U_{i} \delta_{i}^{2} \sqrt{\sigma_{b}^{2}+\lambda^{2} \Lambda_{i}} W_{\Phi}\left(\frac{\lambda \eta_{i}}{\sqrt{\sigma_{b}^{2}+\lambda^{2} \Lambda_{i}}}\right) \mid \mathbf{y}_{i}, \boldsymbol{\theta}\right] \\
& {\widehat{b^{2}}}_{i}=\eta_{i}^{2}+\frac{\lambda^{2}}{\sigma_{b}^{2}} E_{U_{i}}\left(\delta_{i}^{2} \mid \mathbf{y}_{i}, \boldsymbol{\theta}\right)+\frac{2 \lambda \eta_{i}}{\sigma_{b}^{2}} E_{U_{i}}\left[\delta_{i} \sqrt{\sigma_{b}^{2}+\lambda^{2} \Lambda_{i}} W_{\Phi}\left(\frac{\lambda \eta_{i}}{\sqrt{\sigma_{b}^{2}+\lambda^{2} \Lambda_{i}}}\right) \mid \mathbf{y}_{i}, \boldsymbol{\theta}\right] \\
& +E_{U_{i}}\left[\delta_{i} \mid \mathbf{y}_{i}, \boldsymbol{\theta}\right]+\frac{\lambda^{4}}{\sigma_{b}^{4}} E_{U_{i}}\left(\Lambda_{i} \delta_{i}^{2} \mid \mathbf{y}_{i}, \boldsymbol{\theta}\right)-\frac{\lambda^{3} \eta_{i}}{\sigma_{b}^{4}} E_{U_{i}}\left[\delta_{i}^{2} \sqrt{\sigma_{b}^{2}+\lambda^{2} \Lambda_{i}} W_{\Phi}\left(\frac{\lambda \eta_{i}}{\sqrt{\sigma_{b}^{2}+\lambda^{2} \Lambda_{i}}}\right) \mid \mathbf{y}_{i}, \boldsymbol{\theta}\right], \\
& \widehat{t b}_{i}=\lambda \eta_{i}^{2}+\lambda E_{U_{i}}\left(\delta_{i} \mid \mathbf{y}_{i}, \boldsymbol{\theta}\right)+\frac{\lambda^{3}}{\sigma_{b}^{2}} E_{U_{i}}\left(\Lambda_{i} \delta_{i} \mid \mathbf{y}_{i}, \boldsymbol{\theta}\right) \\
& +\eta_{i} E_{U_{i}}\left[\sqrt{\sigma_{b}^{2}+\lambda^{2} \Lambda_{i}} W_{\Phi}\left(\frac{\lambda \eta_{i}}{\sqrt{\sigma_{b}^{2}+\lambda^{2} \Lambda_{i}}}\right) \mid \mathbf{y}_{i}, \boldsymbol{\theta}\right] \\
& \widehat{t_{i}}=\lambda \eta_{i}+E_{U_{i}}\left[\sqrt{\sigma_{b}^{2}+\lambda^{2} \Lambda_{i}} W_{\Phi}\left(\frac{\lambda \eta_{i}}{\sqrt{\sigma_{b}^{2}+\lambda^{2} \Lambda_{i}}}\right) \mid \mathbf{y}_{i}, \boldsymbol{\theta}\right] \mathrm{e} \\
& {\widehat{t^{2}}}_{i}=\lambda^{2} \eta_{i}^{2}+\sigma_{b}^{2}+\lambda^{2} E_{U_{i}}\left(\Lambda_{i} \mid \mathbf{y}_{i}, \boldsymbol{\theta}\right)+\lambda \eta_{i} E_{U_{i}}\left[\sqrt{\sigma_{b}^{2}+\lambda^{2} \Lambda_{i}} W_{\Phi}\left(\frac{\lambda \eta_{i}}{\sqrt{\sigma_{b}^{2}+\lambda^{2} \Lambda_{i}}}\right) \mid \mathbf{y}_{i}, \boldsymbol{\theta}\right]
\end{aligned}
$$

Cálculo de $E_{U_{i}}\left[g\left(U_{i}\right) \mid \mathbf{y}_{i}, \boldsymbol{\theta}\right], g$ funções reais, presentes em (5.24-5.29).

Das equações (5.15) e (5.20) tem-se que

$$
\begin{aligned}
f\left(\mathbf{y}_{i} \mid \boldsymbol{\theta}\right) & =2 \int_{0}^{+\infty} \int_{-\infty}^{+\infty} \phi_{n_{i}}\left(\mathbf{y}_{i} \mid \mathbf{x}_{i}^{\top} \boldsymbol{\beta}, u_{i}^{-1}\left[\sigma_{e}^{2} \mathbb{I}_{n_{i}}+\sigma_{b}^{2} \mathbf{Z}_{i} \mathbf{Z}_{i}^{\top}\right]\right) \phi\left(b_{i} \mid \sigma_{e}^{-2} \Lambda_{i}^{*} \mathbf{Z}_{i}^{\top}\left(\mathbf{y}_{i}-\mathbf{x}_{i}^{\top} \boldsymbol{\beta}\right), \Lambda_{i}\right) \\
& \times \Phi\left(\frac{\lambda b_{i}}{\sigma_{b}}\right) h\left(u_{i} ; \boldsymbol{\tau}\right) d b_{i} d u_{i} \\
& =2 \int_{0}^{+\infty} \phi_{n_{i}}\left(\mathbf{y}_{i} \mid \mathbf{x}_{i}^{\top} \boldsymbol{\beta}, u_{i}^{-1}\left[\sigma_{e}^{2} \mathbb{I}_{n_{i}}+\sigma_{b}^{2} \mathbf{Z}_{i} \mathbf{Z}_{i}^{\top}\right]\right) E_{b_{i} \mid \mathbf{y}_{i}, U_{i}}\left[\Phi\left(\frac{\lambda b_{i}}{\sigma_{b}}\right)\right] h\left(u_{i} ; \boldsymbol{\tau}\right) d u_{i} .(5.30)
\end{aligned}
$$

Pelo Lema 1 de Arellano-Valle et al. (2005a), para $\mu=\sigma_{e}^{-2} \Lambda_{i}^{*} \mathbf{Z}_{i}^{\top}\left(\mathbf{y}_{i}-\mathbf{x}_{i}^{\top} \boldsymbol{\beta}\right)=\eta_{i}, \Sigma=\Lambda_{i}$, $a=0, B=\lambda / \sigma_{b}, \eta=0$ e $\Omega=1$, tem-se que 


$$
E_{b_{i}}\left[\Phi\left(\frac{\lambda b_{i}}{\sigma_{b}}\right) \mid \mathbf{y}_{i}, U_{i}, \boldsymbol{\theta}\right]=\Phi\left(\frac{\lambda \eta_{i}}{\sqrt{\sigma_{b}^{2}+\lambda^{2} \Lambda_{i}}}\right)
$$

Portanto,

$$
f\left(\mathbf{y}_{i} \mid \boldsymbol{\theta}\right)=2 \int_{0}^{+\infty} \phi_{n_{i}}\left(\mathbf{y}_{i} \mid \mathbf{x}_{i}^{\top} \boldsymbol{\beta}, u_{i}^{-1}\left[\sigma_{e}^{2} \mathbb{I}_{n_{i}}+\sigma_{b}^{2} \mathbf{Z}_{i} \mathbf{Z}_{i}^{\top}\right]\right) \Phi\left(\frac{\lambda \eta_{i}}{\sqrt{\sigma_{b}^{2}+\lambda^{2} \Lambda_{i}}}\right) h\left(u_{i} ; \boldsymbol{\tau}\right) d u_{i}
$$

A esperança condicional $E_{U_{i}}\left[g\left(U_{i}\right) \mid \mathbf{y}_{i}, \boldsymbol{\theta}\right]$ pode ser escrita como

$$
E_{U_{i} \mid \mathbf{y}_{i}}\left[g\left(U_{i}\right)\right]=\int g\left(u_{i}\right) f\left(u_{i} \mid \mathbf{y}_{i}, \boldsymbol{\theta}\right) d u_{i}=\frac{\int g\left(u_{i}\right) f\left(\mathbf{y}_{i} \mid u_{i}, \boldsymbol{\theta}\right) h\left(u_{i} ; \boldsymbol{\tau}\right) d u_{i}}{\int f\left(\mathbf{y}_{i} \mid u_{i}, \boldsymbol{\theta}\right) h\left(u_{i} ; \boldsymbol{\tau}\right) d u_{i}}
$$

As integrais acima podem ser obtidas via Monte Carlo, gerando $r$ (suficientemente grande, por exemplo, $\mathrm{r}=1000)$ replicações de $U_{i j} \sim H\left(u_{i} ; \boldsymbol{\tau}\right)$, dadas na Seção 4.2.1, e então

$$
\widehat{g\left(U_{i}\right)}=\frac{\sum_{j=1}^{r} g\left(u_{i j}\right) f\left(\mathbf{y}_{i} \mid u_{i j}, \boldsymbol{\theta}\right)}{\sum_{j=1}^{r} f\left(\mathbf{y}_{i} \mid u_{i j}, \boldsymbol{\theta}\right)}
$$

De acordo com (5.31), $f\left(\mathbf{y}_{i} \mid u_{i}, \boldsymbol{\theta}\right)$ é dada por

$$
f\left(\mathbf{y}_{i} \mid u_{i}, \boldsymbol{\theta}\right)=2 \phi_{n_{i}}\left(\mathbf{y}_{i} \mid \mathbf{x}_{i}^{\top} \boldsymbol{\beta}, u_{i}^{-1}\left[\sigma_{e}^{2} \mathbb{I}_{n_{i}}+\sigma_{b}^{2} \mathbf{Z}_{i} \mathbf{Z}_{i}^{\top}\right]\right) \Phi\left(\frac{\lambda \eta_{i}}{\sqrt{\sigma_{b}^{2}+\lambda^{2} \Lambda_{i}}}\right)
$$

ou seja, $\mathbf{y}_{i} \mid u_{i}, \boldsymbol{\theta} \sim N A_{n_{i}}\left(\boldsymbol{\mu}_{i}, \boldsymbol{\Sigma}_{i}, \boldsymbol{\lambda}_{i}\right), \operatorname{com} \boldsymbol{\mu}_{i}=\mathbf{x}_{i}^{\top} \boldsymbol{\beta}, \boldsymbol{\Sigma}_{i}=u_{i}^{-1}\left[\sigma_{e}^{2} \mathbb{I}_{n_{i}}+\sigma_{b}^{2} \mathbf{Z}_{i} \mathbf{Z}_{i}^{\top}\right]$ e $\boldsymbol{\lambda}_{i}=\frac{\lambda u_{i}^{-1 / 2} \Lambda_{i}^{*}}{\sigma_{e}^{2} \sqrt{\sigma_{b}^{2}+\lambda^{2} \Lambda_{i}}}\left[\sigma_{e}^{2} \mathbb{I}_{n_{i}}+\sigma_{b}^{2} \mathbf{Z}_{i} \mathbf{Z}_{i}^{\top}\right]^{1 / 2} \mathbf{Z}_{i}$. Esse procedimento foi adotado por Walker (1996) em um algoritmo EM para modelos de efeitos aleatórios não-lineares.

\subsubsection{O modelo misto normal assimétrico}

Como um caso particular do MLMMENA, apresentamos o modelo linear misto normal assimétrico (MLMNA), representado hierarquicamente da seguinte forma:

$$
\begin{aligned}
\mathbf{Y}_{i} \mid b_{i} & \sim N_{n_{i}}\left(\mathbf{x}_{i}^{\top} \boldsymbol{\beta}+\mathbf{Z}_{i} b_{i}, \sigma_{e}^{2} \mathbb{I}_{n_{i}}\right) \mathrm{e} \\
b_{i} & \sim N A\left(0, \sigma_{b}^{2}, \lambda\right), i=1, \ldots, m .
\end{aligned}
$$


A função densidade do modelo (5.33) é dada por

$$
f\left(\mathbf{y}_{i}\right)=2 \int_{-\infty}^{+\infty} \phi_{n_{i}}\left(\mathbf{y}_{i} \mid \mathbf{x}_{i}^{\top} \boldsymbol{\beta}+b_{i} \mathbf{Z}_{i}, \sigma_{e}^{2} \mathbb{I}_{n_{i}}\right) \phi\left(b_{i} \mid 0, \sigma_{b}^{2}\right) \Phi\left(\frac{\lambda b_{i}}{\sigma_{b}}\right) d b_{i}
$$

\section{Algoritmo EM:}

Passo E: Dado $\boldsymbol{\theta}=\widehat{\boldsymbol{\theta}}$, calcule para $i=1, \ldots, m, \widehat{b}_{i}, \widehat{b}^{2}{ }_{i}, \widehat{t}^{2}{ }_{i}$ e $\widehat{t b}_{i}$ usando (5.35)-(5.39).

Passo M: Atualize $\widehat{\boldsymbol{\theta}}$ maximizando $Q(\boldsymbol{\theta} \mid \widehat{\boldsymbol{\theta}})$ em relação a $\boldsymbol{\theta}$, levando às seguintes expressões:

$$
\begin{aligned}
\widehat{\boldsymbol{\beta}} & =\left(\mathbf{X}^{\top} \mathbf{X}\right)^{-1} \mathbf{X}^{\top}\left(\mathbf{Y}-\mathbf{D}\left(\mathbf{b}^{*}\right) \mathbf{Z}\right) \\
\widehat{\lambda} & =\frac{\sum_{i=1}^{m} \widehat{t b}_{i}}{\sum_{i=1}^{m}{\widehat{b^{2}}}_{i}} \\
\widehat{\sigma_{e}^{2}} & =\frac{1}{N} \sum_{i=1}^{m}\left(\left(\mathbf{y}_{i}-\mathbf{x}_{i}^{\top} \boldsymbol{\beta}\right)^{\top}\left(\mathbf{y}_{i}-\mathbf{x}_{i}^{\top} \boldsymbol{\beta}\right)-2 \widehat{b}_{i} \mathbf{Z}_{i}^{\top}\left(\mathbf{y}_{i}-\mathbf{x}_{i}^{\top} \boldsymbol{\beta}\right)+{\widehat{b^{2}}}_{i} \mathbf{Z}_{i}^{\top} \mathbf{Z}_{i}\right) \mathrm{e} \\
\widehat{\sigma_{b}^{2}} & =\frac{1}{2 m} \sum_{i=1}^{m}\left(\left(1+\lambda^{2}\right){\widehat{b^{2}}}_{i}+\widehat{t}^{2}{ }_{i}-2 \lambda \widehat{t b}_{i}\right),
\end{aligned}
$$

onde $\mathbf{X}=\left(\mathbf{x}_{1}^{\top}, \ldots, \mathbf{x}_{m}^{\top}\right)^{\top}$ de dimensão $N \times p, \mathbf{Z}=\left(\mathbf{Z}_{1}^{\top}, \ldots, \mathbf{Z}_{m}^{\top}\right)^{\top}$ de dimensão $N \times 1$, $N=\sum_{i=1}^{m} n_{i}, \widehat{\mathbf{b}}^{*}=\left[\widehat{b}_{1} \mathbf{1}_{n_{1}}^{\top}, \ldots, \widehat{b}_{m} \mathbf{1}_{n_{m}}^{\top}\right]^{\top}, \mathbf{D}\left(\widehat{\mathbf{b}}^{*}\right)$ é a matriz diagonal com os elementos do vetor $\widehat{\mathbf{b}}^{*}$ e $\mathbf{1}_{k}$ é um vetor coluna $k$-dimensional de 1 's, $k>0$. Os estimadores das variáveis latentes são dados por

$$
\begin{gathered}
\widehat{b}_{i}=\eta_{i}+\frac{\lambda \delta_{i}}{\sigma_{b}^{2}} \sqrt{\sigma_{b}^{2}+\lambda^{2} \Lambda_{i}^{*}} W_{\Phi}\left(\frac{\lambda \eta_{i}}{\sqrt{\sigma_{b}^{2}+\lambda^{2} \Lambda_{i}^{*}}}\right) \\
\widehat{b}^{2}{ }_{i}=\eta_{i}^{2}+\delta_{i}+\frac{2 \lambda \eta_{i} \delta_{i}}{\sigma_{b}^{2}} \sqrt{\sigma_{b}^{2}+\lambda^{2} \Lambda_{i}^{*}} W_{\Phi}\left(\frac{\lambda \eta_{i}}{\sqrt{\sigma_{b}^{2}+\lambda^{2} \Lambda_{i}^{*}}}\right) \\
+\frac{\lambda^{2} \delta_{i}^{2}}{\sigma_{b}^{4}}\left[\sigma_{b}^{2}+\lambda^{2} \Lambda_{i}^{*}-\lambda \eta_{i} \sqrt{\sigma_{b}^{2}+\lambda^{2} \Lambda_{i}^{*}} W_{\Phi}\left(\frac{\lambda \eta_{i}}{\sqrt{\sigma_{b}^{2}+\lambda^{2} \Lambda_{i}^{*}}}\right)\right] \\
\widehat{t}_{i}=\lambda \eta_{i}+\sqrt{\sigma_{b}^{2}+\lambda^{2} \Lambda_{i}^{*}} W_{\Phi}\left(\frac{\lambda \eta_{i}}{\sqrt{\sigma_{b}^{2}+\lambda^{2} \Lambda_{i}^{*}}}\right) \\
\widehat{\lambda}_{i}^{2}+\sigma_{b}^{2}+\lambda^{2} \Lambda_{i}^{*}+\lambda \eta_{i} \sqrt{\sigma_{b}^{2}+\lambda^{2} \Lambda_{i}^{*}} W_{\Phi}\left(\frac{\lambda \eta_{i}}{\sqrt{\sigma_{b}^{2}+\lambda^{2} \Lambda_{i}^{*}}}\right) \mathrm{e} \\
\widehat{T}_{i}+\frac{\lambda \delta_{i}}{\sigma_{b}^{2}}\left(\widehat{t}^{2}{ }_{i}-\lambda \eta_{i} \widehat{t}_{i}\right)
\end{gathered}
$$




\subsection{Matriz de Informação Observada}

A função densidade de $\mathbf{y}_{i}$ na equação (5.31) envolve uma integral, o que dificulta o cálculo da matriz de informação (MI). Por outro lado, de acordo com Louis (1982), quando se utiliza o algoritmo EM para o cálculo das estimativas de máxima verossimilhança em problemas com dados incompletos, pode-se obter uma aproximação da MI, envolvendo primeiras e segundas derivadas da função log-verossimilhança dos dados completos. A proposta de MI de Louis (1982) é da seguinte forma

$$
\mathbf{I}(\boldsymbol{\theta}) \cong-\ddot{Q}_{\boldsymbol{\theta}}(\widehat{\boldsymbol{\theta}})-E\left[S\left(\mathbf{y}_{c}, \boldsymbol{\theta}\right) S^{\top}\left(\mathbf{y}_{c}, \boldsymbol{\theta}\right)\right]+S^{*}(\mathbf{y}, \boldsymbol{\theta}) S^{* \top}(\mathbf{y}, \boldsymbol{\theta})
$$

onde $\ddot{Q}_{\boldsymbol{\theta}}(\widehat{\boldsymbol{\theta}})=\left.\frac{\partial^{2} Q(\boldsymbol{\theta} \mid \widehat{\boldsymbol{\theta}})}{\partial \boldsymbol{\theta} \partial \boldsymbol{\theta}^{\top}}\right|_{\boldsymbol{\theta}=\widehat{\boldsymbol{\theta}}}, S\left(\mathbf{y}_{c}, \boldsymbol{\theta}\right)=\frac{\partial \ell_{c}\left(\boldsymbol{\theta} \mid \mathbf{y}_{c}\right)}{\partial \boldsymbol{\theta}}$ e $S^{*}(\mathbf{y}, \widehat{\boldsymbol{\theta}})=\frac{\partial Q(\boldsymbol{\theta} \mid \boldsymbol{\theta})}{\partial \boldsymbol{\theta}}$. Avaliado em $\widehat{\boldsymbol{\theta}}$, o terceiro elemento de $\mathbf{I}(\boldsymbol{\theta})$ é igual a zero. A matriz hessiana $\ddot{Q}_{\boldsymbol{\theta}}(\widehat{\boldsymbol{\theta}})$ encontra-se na Seção 5.5 , sendo necessário apenas o cálculo de $E\left[S\left(\mathbf{y}_{c}, \boldsymbol{\theta}\right) S^{\top}\left(\mathbf{y}_{c}, \boldsymbol{\theta}\right)\right]$. Os elementos dessa matriz, bem como os estimadores das variáveis latentes são descritos a seguir.

Seja $I^{1}(\theta)=E\left[S\left(\mathbf{y}_{c}, \boldsymbol{\theta}\right) S^{\top}\left(\mathbf{y}_{c}, \boldsymbol{\theta}\right)\right]$, a matriz simétrica com elementos

$$
I^{1}(\boldsymbol{\theta})=\left(\begin{array}{cccc}
I_{\boldsymbol{\beta} \boldsymbol{\beta}}^{1} & I_{\boldsymbol{\beta}_{\sigma_{e}^{2}}}^{1} & I_{\boldsymbol{\beta}_{\sigma_{b}^{2}}}^{1} & I_{\boldsymbol{\beta}_{\lambda}}^{1} \\
& I_{\sigma_{e}^{2} \sigma_{e}^{2}}^{1} & I_{\sigma_{e}^{2} \sigma_{b}^{2}}^{1} & I_{\sigma_{e}^{2} \lambda}^{1} \\
& & I_{\sigma_{b}^{2} \sigma_{b}^{2}}^{1} & I_{\sigma_{b}^{2} \lambda}^{1} \\
& & & I_{\lambda \lambda}^{1}
\end{array}\right),
$$

sendo que

$$
\begin{aligned}
I_{\boldsymbol{\beta} \boldsymbol{\beta}}^{1}= & \frac{1}{\sigma_{e}^{4}} \sum_{i=1}^{m} \mathbf{x}_{i}\left[\widehat{U^{2}}{ }_{i}\left(\mathbf{y}_{i}-\mathbf{x}_{i}^{\top} \boldsymbol{\beta}\right)\left(\mathbf{y}_{i}-\mathbf{x}_{i}^{\top} \boldsymbol{\beta}\right)^{\top}-\widehat{U^{2} b_{i}}\left(\mathbf{y}_{i}-\mathbf{x}_{i}^{\top} \boldsymbol{\beta}\right) \mathbf{Z}_{i}^{\top}\right. \\
& \left.-\widehat{U^{2} b_{i}} \mathbf{Z}_{i}\left(\mathbf{y}_{i}-\mathbf{x}_{i}^{\top} \boldsymbol{\beta}\right)^{\top}+\widehat{U^{2} b^{2}}{ }_{i} \mathbf{Z}_{i} \mathbf{Z}_{i}^{\top}\right] \mathbf{x}_{i}^{\top}, \\
I_{\boldsymbol{\beta}_{\sigma_{e}^{2}}}^{1}= & \frac{1}{2 \sigma_{e}^{4}} \sum_{i=1}^{m} \mathbf{x}_{i}\left[\frac{1}{\sigma_{e}^{2}}\left(\mathbf{y}_{i}-\mathbf{x}_{i}^{\top} \boldsymbol{\beta}\right)\left(\widehat{U^{2}}{ }_{i} Q_{i}(\boldsymbol{\beta})-2 \widehat{U^{2} b_{i}} \mathbf{Z}_{i}^{\top}\left(\mathbf{y}_{i}-\mathbf{x}_{i}^{\top} \boldsymbol{\beta}\right)+\widehat{U^{2} b^{2}}{ }_{i} \mathbf{Z}_{i}^{\top} \mathbf{Z}_{i}\right)\right. \\
& -\frac{1}{\sigma_{e}^{2}} \mathbf{Z}_{i}\left(\widehat{U^{2} b_{i}} Q_{i}(\boldsymbol{\beta})-2 \widehat{U^{2} b^{2}}{ }_{i} \mathbf{Z}_{i}^{\top}\left(\mathbf{y}_{i}-\mathbf{x}_{i}^{\top} \boldsymbol{\beta}\right)+\widehat{U^{2} b^{3}}{ }_{i} \mathbf{Z}_{i}^{\top} \mathbf{Z}_{i}\right) \\
& \left.-n_{i} \widehat{U}_{i}\left(\mathbf{y}_{i}-\mathbf{x}_{i}^{\top} \boldsymbol{\beta}\right)+n_{i} \widehat{U b} \mathbf{Z}_{i}\right]
\end{aligned}
$$

Ferreira, C. S. 


$$
\begin{aligned}
& I_{\boldsymbol{\beta}_{\sigma_{b}^{2}}}^{1}=\frac{1}{\sigma_{e}^{2} \sigma_{b}^{2}} \sum_{i=1}^{m} \mathbf{x}_{i}\left[\widehat{U b}_{i} \mathbf{Z}_{i}+\frac{1}{2 \sigma_{b}^{2}}\left(\mathbf{y}_{i}-\mathbf{x}_{i}^{\top} \boldsymbol{\beta}\right)\left({\widehat{U b^{2}}}_{i}+{\widehat{U t^{2}}}_{i}-2 \lambda \widehat{U t b}_{i}+\lambda^{2}{\widehat{U b^{2}}}_{i}\right)\right. \\
& \left.-\widehat{U}_{i}\left(\mathbf{y}_{i}-\mathbf{x}_{i}^{\top} \boldsymbol{\beta}\right)-\frac{1}{2 \sigma_{b}^{2}} \mathbf{Z}_{i}\left({\widehat{U^{2} b^{3}}}_{i}+{\widehat{U t^{2}}}_{i}-2 \lambda \widehat{U t b^{2}}{ }_{i}+\lambda^{2}{\widehat{U b^{3}}}_{i}\right)\right] \\
& I_{\boldsymbol{\beta}_{\lambda}}^{1}=\frac{1}{\sigma_{e}^{2} \sigma_{b}^{2}} \sum_{i=1}^{m} \mathbf{x}_{i}\left[\left(\widehat{U t b}_{i}-\lambda{\widehat{U b^{2}}}_{i}\right)\left(\mathbf{y}_{i}-\mathbf{x}_{i}^{\top} \boldsymbol{\beta}\right)-\left({\widehat{U t b^{2}}}_{i}-\lambda{\widehat{U b^{3}}}_{i}\right) \mathbf{Z}_{i}\right] \\
& I_{\sigma_{e}^{2} \sigma_{e}^{2}}^{1}=\sum_{i=1}^{m}\left\{\frac{n_{i}^{2}}{4 \sigma_{e}^{4}}-\frac{n_{i}}{2 \sigma_{e}^{6}}\left(\widehat{U}_{i} Q_{i}(\boldsymbol{\beta})-2 \widehat{U b}_{i} \mathbf{Z}_{i}^{\top}\left(\mathbf{y}_{i}-\mathbf{x}_{i}^{\top} \boldsymbol{\beta}\right)+{\widehat{U b^{2}}}_{i} \mathbf{Z}_{i}^{\top} \mathbf{Z}_{i}\right)\right. \\
& +\frac{1}{4 \sigma_{e}^{8}}\left[\widehat{U^{2}}{ }_{i} Q_{i}^{2}(\boldsymbol{\beta})+4 \widehat{U^{2} b^{2}}{ }_{i}\left[\mathbf{Z}_{i}^{\top}\left(\mathbf{y}_{i}-\mathbf{x}_{i}^{\top} \boldsymbol{\beta}\right)\right]^{2}+\widehat{U^{2} b^{4}}{ }_{i}\left[\mathbf{Z}_{i}^{\top} \mathbf{Z}_{i}\right]^{2}\right. \\
& -4{\widehat{U^{2}}}_{i} Q_{i}(\boldsymbol{\beta}) \mathbf{Z}_{i}^{\top}\left(\mathbf{y}_{i}-\mathbf{x}_{i}^{\top} \boldsymbol{\beta}\right)+2 \widehat{U^{2} b^{2}}{ }_{i} Q_{i}(\boldsymbol{\beta}) \mathbf{Z}_{i}^{\top} \mathbf{Z}_{i} \\
& \left.\left.-4 \widehat{U^{2} b^{3}} \mathbf{Z}_{i}^{\top}\left(\mathbf{y}_{i}-\mathbf{x}_{i}^{\top} \boldsymbol{\beta}\right) \mathbf{Z}_{i}^{\top} \mathbf{Z}_{i}\right]\right\} \\
& I_{\sigma_{e}^{2} \sigma_{b}^{2}}^{1}=\sum_{i=1}^{m}\left\{\frac{n_{i}}{2 \sigma_{e}^{2} \sigma_{b}^{2}}-\frac{n_{i}}{4 \sigma_{e}^{2} \sigma_{b}^{4}}\left({\widehat{U b^{2}}}_{i}+\widehat{t}^{2}{ }_{i}-2 \lambda \widehat{t b}_{i}+\lambda^{2}{\widehat{b^{2}}}_{i}\right)\right. \\
& -\frac{1}{2 \sigma_{e}^{4} \sigma_{b}^{2}}\left(\widehat{U}_{i} Q_{i}(\boldsymbol{\beta})-2 \widehat{U b}_{i} \mathbf{Z}_{i}^{\top}\left(\mathbf{y}_{i}-\mathbf{x}_{i}^{\top} \boldsymbol{\beta}\right)+\widehat{U b^{2}}{ }_{i} \mathbf{Z}_{i}^{\top} \mathbf{Z}_{i}\right) \\
& +\frac{1}{4 \sigma_{e}^{4} \sigma_{b}^{4}}\left[\left({\widehat{U b^{2} b^{2}}}_{i}+{\widehat{U t^{2}}}_{i}-2 \lambda \widehat{U t b}_{i}+\lambda^{2}{\widehat{U b^{2}}}_{i}\right) Q_{i}(\boldsymbol{\beta})\right. \\
& -2\left({\widehat{U U^{2} b^{3}}}_{i}+{\widehat{U t^{2}}}_{i}-2 \lambda \widehat{U t b^{2}}{ }_{i}+\lambda^{2}{\widehat{U b^{3}}}_{i}\right) \mathbf{Z}_{i}^{\top}\left(\mathbf{y}_{i}-\mathbf{x}_{i}^{\top} \boldsymbol{\beta}\right) \\
& \left.+\left({\widehat{U b^{2}}}_{i}+{\widehat{U t^{2} b^{2}}}_{i}-2 \lambda{\widehat{U t b^{3}}}_{i}+\lambda^{2}{\widehat{U b^{4}}}_{i}\right) \mathbf{Z}_{i}^{\top} \mathbf{Z}_{i}\right\} \\
& I_{\sigma_{e}^{2} \lambda}^{1}=\frac{1}{2 \sigma_{e}^{2} \sigma_{b}^{2}} \sum_{i=1}^{m}\left\{\frac { 1 } { \sigma _ { e } ^ { 2 } } \left[\left(\widehat{U t b}_{i}-\lambda{\widehat{U b^{2}}}_{i}\right) Q_{i}(\boldsymbol{\beta})+\left({\widehat{U t b^{3}}}_{i}-\lambda{\widehat{U b^{4}}}_{i}\right) \mathbf{Z}_{i}^{\top} \mathbf{Z}_{i}\right.\right. \\
& \left.\left.-2\left({\widehat{U t b^{2}}}_{i}-\lambda{\widehat{U b^{3}}}_{i}\right) \mathbf{Z}_{i}^{\top}\left(\mathbf{y}_{i}-\mathbf{x}_{i}^{\top} \boldsymbol{\beta}\right)\right]-n_{i}\left(\widehat{t b}_{i}-\lambda{\widehat{b^{2}}}_{i}\right)\right\} \\
& I_{\sigma_{b}^{2} \sigma_{b}^{2}}^{1}=\sum_{i=1}^{m}\left[\frac{1}{\sigma_{b}^{4}}-\frac{1}{\sigma_{b}^{6}}\left({\widehat{U b^{2}}}_{i}+\widehat{t}^{2}{ }_{i}-2 \lambda \widehat{t b}_{i}+\lambda^{2}{\widehat{b^{2}}}_{i}\right)\right. \\
& +\frac{1}{4 \sigma_{b}^{8}}\left({\widehat{U^{2} b^{4}}}_{i}+\widehat{t}^{4}{ }_{i}+6 \lambda^{2}{\widehat{t^{2} b^{2}}}_{i}+\lambda^{4}{\widehat{b^{4}}}_{i}+2 \widehat{U t^{2} b^{2}}{ }_{i}\right. \\
& \left.\left.-4 \lambda{\widehat{U t b^{3}}}_{i}-4 \lambda \widehat{t t}^{3} b_{i}-4 \lambda^{3}{\widehat{t b^{3}}}_{i}+2 \lambda^{2}{\widehat{U b^{4}}}_{i}\right)\right]
\end{aligned}
$$




$$
\begin{aligned}
& I_{\sigma_{b}^{2} \lambda}^{1}=\frac{1}{\sigma_{b}^{4}} \sum_{i=1}^{m}\left[\lambda{\widehat{b^{2}}}_{i}-\widehat{t b}_{i}+\frac{1}{2 \sigma_{b}^{2}}\left({\widehat{U t b^{3}}}_{i}-\lambda{\widehat{U b^{4}}}_{i}+\widehat{t}^{3 b_{i}}{ }_{i}-\lambda{\widehat{t^{2} b^{2}}}_{i}\right.\right.
\end{aligned}
$$

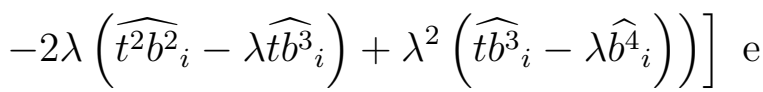

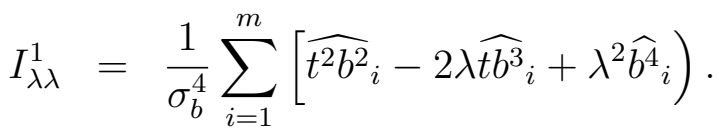

O cálculo dos estimadores das variáveis latentes das equações acima será da mesma forma como na seção 5.3.1. Dado que $T_{i} \mid \mathbf{y}_{i}, U_{i}, \boldsymbol{\theta} \sim N T\left(\lambda \eta_{i}, \sigma_{b}^{2}+\lambda^{2} \Lambda_{i}\right), \eta_{i}=\Lambda_{i}^{*} \sigma_{e}^{-2} \mathbf{Z}_{i}^{\top}\left(\mathbf{y}_{i}-\mathbf{x}_{i}^{\top} \boldsymbol{\beta}\right)$, $\Lambda_{i}^{*}=\left(\sigma_{b}^{-2}+\sigma_{e}^{-2} \mathbf{Z}_{i}^{\top} \mathbf{Z}_{i}\right)^{-1}, \Lambda_{i}=U_{i}^{-1}\left(\sigma_{b}^{-2}+\sigma_{e}^{-2} \mathbf{Z}_{i}^{\top} \mathbf{Z}_{i}\right)^{-1}$, tem-se que

$$
\begin{gathered}
\widehat{T}\left(U_{i}\right)=E_{T_{i} \mid \mathbf{y}_{i}, U_{i},}, \boldsymbol{\theta}\left[T_{i}\right]=\lambda \eta_{i}+\sqrt{\sigma_{b}^{2}+\lambda^{2} \Lambda_{i}} W_{\Phi}\left(\frac{\lambda \eta_{i}}{\sqrt{\sigma_{b}^{2}+\lambda^{2} \Lambda_{i}}}\right), \\
\widehat{T^{2}}\left(U_{i}\right)=E_{T_{i} \mid \mathbf{y}_{i}, U_{i}, \boldsymbol{\theta}}\left[T_{i}^{2}\right]=\lambda^{2} \eta_{i}^{2}+\sigma_{b}^{2}+\lambda^{2} \Lambda_{i}+\lambda \eta_{i} \sqrt{\sigma_{b}^{2}+\lambda^{2} \Lambda_{i}} W_{\Phi}\left(\frac{\lambda \eta_{i}}{\sqrt{\sigma_{b}^{2}+\lambda^{2} \Lambda_{i}}}\right),
\end{gathered}
$$

$$
\begin{aligned}
& \widehat{T^{3}}\left(U_{i}\right)=E_{T_{i} \mid \mathbf{y}_{i}, U_{i}, \boldsymbol{\theta}}\left[T_{i}^{3}\right]=\lambda^{3} \eta_{i}^{3}+3 \lambda \eta_{i}\left(\sigma_{b}^{2}+\lambda^{2} \Lambda_{i}\right) \\
& +\left[\lambda^{2} \eta_{i}^{2} \sqrt{\sigma_{b}^{2}+\lambda^{2} \Lambda_{i}}+2\left(\sigma_{b}^{2}+\lambda^{2} \Lambda_{i}\right)^{3 / 2}\right] W_{\Phi}\left(\frac{\lambda \eta_{i}}{\sqrt{\sigma_{b}^{2}+\lambda^{2} \Lambda_{i}}}\right) \mathrm{e}
\end{aligned}
$$

$$
\begin{aligned}
\widehat{T^{4}}\left(U_{i}\right)=E_{T_{i} \mid \mathbf{y}_{i}, U_{i},}, \boldsymbol{\theta}^{\left[T_{i}^{4}\right]=} & \lambda^{4} \eta_{i}^{4}+6 \lambda^{2} \eta_{i}^{2}\left(\sigma_{b}^{2}+\lambda^{2} \Lambda_{i}\right)+3\left(\sigma_{b}^{2}+\lambda^{2} \Lambda_{i}\right)^{2} \\
& +\left[\lambda^{3} \eta_{i}^{3} \sqrt{\sigma_{b}^{2}+\lambda^{2} \Lambda_{i}}+5 \lambda \eta_{i}\left(\sigma_{b}^{2}+\lambda^{2} \Lambda_{i}\right)^{3 / 2}\right] W_{\Phi}\left(\frac{\lambda \eta_{i}}{\sqrt{\sigma_{b}^{2}+\lambda^{2} \Lambda_{i}}}\right) .
\end{aligned}
$$

Para fins de praticidade em escrever os estimadores das variáveis latentes e devido ao número de vezes em que aparecem nas equações, define-se os seguintes elementos:

$$
\begin{gathered}
\mu_{1}\left(U_{i}\right)=E_{T_{i} \mid \mathbf{y}_{i}, U_{i},}, \boldsymbol{\theta} \\
\mu_{2}\left(U_{i}\right)=E_{T_{i} \mid \mathbf{y}_{i}, U_{i}}, \boldsymbol{\theta} \\
{\left[T_{i}-\lambda \eta_{i}\right]^{2}=\widehat{T^{2}}\left(U_{i}\right)-2 \lambda \eta_{i} \widehat{T}\left(U_{i}\right)+\lambda^{2} \eta_{i}^{2},} \\
\mu_{3}\left(U_{i}\right)=E_{T_{i} \mid \mathbf{y}_{i}, U_{i}, \boldsymbol{\theta}}\left[T_{i}-\lambda \eta_{i}\right]^{3}=\widehat{T^{3}}\left(U_{i}\right)-3 \lambda \eta_{i} \widehat{T^{2}}\left(U_{i}\right)+3 \lambda^{2} \eta_{i}^{2} \widehat{T}\left(U_{i}\right)-\lambda^{3} \eta_{i}^{3}, \\
\mu_{4}\left(U_{i}\right)=E_{T_{i} \mid \mathbf{y}_{i}, U_{i}, \boldsymbol{\theta}}\left[T_{i}-\lambda \eta_{i}\right]^{4}=\widehat{T^{4}}\left(U_{i}\right)-4 \lambda \eta_{i} \widehat{T^{3}}\left(U_{i}\right)+6 \lambda^{2} \eta_{i}^{2} \widehat{T^{2}}\left(U_{i}\right)-4 \lambda^{3} \eta_{i}^{3} \widehat{T}\left(U_{i}\right)+\lambda^{4} \eta_{i}^{4} .
\end{gathered}
$$

Ferreira, C. S. 
Dado que $b_{i} \mid \mathbf{y}_{i}, t_{i}, U_{i}, \boldsymbol{\theta} \sim N\left(\eta_{i}+\frac{\lambda \delta_{i}}{\sigma_{b}^{2}}\left(t_{i}-\lambda \eta_{i}\right), \delta_{i}\right), \delta_{i}=\left(\Lambda_{i}^{-1}+\lambda^{2} \sigma_{b}^{-2}\right)^{-1}$, seguem os estimadores das variáveis latentes, necessários para o cálculo da matriz de informação observada aproximada:

$$
\begin{aligned}
& {\widehat{U^{2} b_{i}}}_{i}=\eta_{i}{\widehat{U^{2}}}_{i}+\frac{\lambda}{\sigma_{b}^{2}} E_{U_{i} \mid \mathbf{y}_{i}, \boldsymbol{\theta}}\left[U_{i}^{2} \delta_{i} \mu_{1}\left(U_{i}\right)\right] \\
& \widehat{U^{2} b^{2}}{ }_{i}=\eta_{i}^{2}{\widehat{U^{2}}}_{i}+\frac{2 \lambda \eta_{i}}{\sigma_{b}^{2}} E_{U_{i} \mid \mathbf{y}_{i},} \boldsymbol{\theta}\left[U_{i}^{2} \delta_{i} \mu_{1}\left(U_{i}\right)\right]+\frac{\lambda^{2}}{\sigma_{b}^{4}} E_{U_{i} \mid \mathbf{y}_{i}, \boldsymbol{\theta}}\left[U_{i}^{2} \delta_{i}^{2} \mu_{2}\left(U_{i}\right)\right]+E_{U_{i} \mid \mathbf{y}_{i}, \boldsymbol{\theta}}\left[U_{i}^{2} \delta_{i}\right], \\
& \widehat{U^{2} b^{3}}{ }_{i}=\eta_{i}^{3}{\widehat{U^{2}}}_{i}+\frac{3 \lambda}{\sigma_{b}^{2}} E_{U_{i} \mid \mathbf{y}_{i},} \boldsymbol{\theta}\left[U_{i}^{2}\left(\eta_{i}^{2}+\delta_{i}\right) \delta_{i} \mu_{1}\left(U_{i}\right)\right]+3 \eta_{i} E_{U_{i} \mid \mathbf{y}_{i},}, \boldsymbol{\theta}\left[U_{i}^{2} \delta_{i}\right] \\
& +\frac{3 \lambda^{2} \eta_{i}}{\sigma_{b}^{4}} E_{U_{i} \mid \mathbf{y}_{i},} \boldsymbol{\theta}\left[U_{i}^{2} \delta_{i}^{2} \mu_{2}\left(U_{i}\right)\right]+\frac{\lambda^{3}}{\sigma_{b}^{6}} E_{U_{i} \mid \mathbf{y}_{i}, \boldsymbol{\theta}}\left[U_{i}^{2} \delta_{i}^{3} \mu_{3}\left(U_{i}\right)\right], \\
& \widehat{U b^{3}}{ }_{i}=\eta_{i}^{3} \widehat{U}_{i}+\frac{3 \lambda}{\sigma_{b}^{2}} E_{U_{i} \mid \mathbf{y}_{i}, \boldsymbol{\theta}}\left[U_{i}\left(\eta_{i}^{2}+\delta_{i}\right) \delta_{i} \mu_{1}\left(U_{i}\right)\right]+3 \eta_{i} E_{U_{i} \mid \mathbf{y}_{i}, \boldsymbol{\theta}}\left[U_{i} \delta_{i}\right] \\
& +\frac{3 \lambda^{2} \eta_{i}}{\sigma_{b}^{4}} E_{U_{i} \mid \mathbf{y}_{i},} \boldsymbol{\theta}\left[U_{i} \delta_{i}^{2} \mu_{2}\left(U_{i}\right)\right]+\frac{\lambda^{3}}{\sigma_{b}^{6}} E_{U_{i} \mid \mathbf{y}_{i}, \boldsymbol{\theta}}\left[U_{i} \delta_{i}^{3} \mu_{3}\left(U_{i}\right)\right], \\
& \widehat{U^{2} b^{4}}{ }_{i}=\eta_{i}^{4}{\widehat{U^{2}}}_{i}+\frac{4 \lambda \eta_{i}}{\sigma_{b}^{2}} E_{U_{i} \mid \mathbf{y}_{i}, \boldsymbol{\theta}}\left[U_{i}^{2}\left(\eta_{i}^{2}+3 \delta_{i}\right) \delta_{i} \mu_{1}\left(U_{i}\right)\right]+\frac{6 \lambda^{2}}{\sigma_{b}^{4}} E_{U_{i} \mid \mathbf{y}_{i},} \boldsymbol{\theta}\left[U_{i}^{2}\left(\eta_{i}^{2}+\delta_{i}\right) \delta_{i}^{2} \mu_{2}\left(U_{i}\right)\right] \\
& +\frac{4 \lambda^{3} \eta_{i}}{\sigma_{b}^{6}} E_{U_{i} \mid \mathbf{y}_{i}, \boldsymbol{\theta}}\left[U_{i}^{2} \delta_{i}^{3} \mu_{3}\left(U_{i}\right)\right]+\frac{\lambda^{4}}{\sigma_{b}^{8}} E_{U_{i} \mid \mathbf{y}_{i}, \boldsymbol{\theta}}\left[U_{i}^{2} \delta_{i}^{4} \mu_{4}\left(U_{i}\right)\right]+3 E_{U_{i} \mid \mathbf{y}_{i}, \boldsymbol{\theta}}\left[U_{i}^{2} \delta_{i}^{2}\right], \\
& {\widehat{U b^{4}}}_{i}=\eta_{i}^{4} \widehat{U}_{i}+\frac{4 \lambda \eta_{i}}{\sigma_{b}^{2}} E_{U_{i} \mid \mathbf{y}_{i}, \boldsymbol{\theta}}\left[U_{i}\left(\eta_{i}^{2}+3 \delta_{i}\right) \delta_{i} \mu_{1}\left(U_{i}\right)\right]+\frac{6 \lambda^{2}}{\sigma_{b}^{4}} E_{U_{i} \mid \mathbf{y}_{i}, \boldsymbol{\theta}}\left[U_{i}\left(\eta_{i}^{2}+\delta_{i}\right) \delta_{i}^{2} \mu_{2}\left(U_{i}\right)\right] \\
& +\frac{4 \lambda^{3} \eta_{i}}{\sigma_{b}^{6}} E_{U_{i} \mid \mathbf{y}_{i},} \boldsymbol{\theta}\left[U_{i} \delta_{i}^{3} \mu_{3}\left(U_{i}\right)\right]+\frac{\lambda^{4}}{\sigma_{b}^{8}} E_{U_{i} \mid \mathbf{y}_{i}, \boldsymbol{\theta}}\left[U_{i} \delta_{i}^{4} \mu_{4}\left(U_{i}\right)\right]+3 E_{U_{i} \mid \mathbf{y}_{i}, \boldsymbol{\theta}}\left[U_{i} \delta_{i}^{2}\right], \\
& {\widehat{b^{4}}}_{i}=\eta_{i}^{4}+\frac{4 \lambda \eta_{i}}{\sigma_{b}^{2}} E_{U_{i} \mid \mathbf{y}_{i}, \boldsymbol{\theta}}\left[\left(\eta_{i}^{2}+3 \delta_{i}\right) \delta_{i} \mu_{1}\left(U_{i}\right)\right]+\frac{6 \lambda^{2}}{\sigma_{b}^{4}} E_{U_{i} \mid \mathbf{y}_{i},} \boldsymbol{\theta}\left[\left(\eta_{i}^{2}+\delta_{i}\right) \delta_{i}^{2} \mu_{2}\left(U_{i}\right)\right] \\
& +\frac{4 \lambda^{3} \eta_{i}}{\sigma_{b}^{6}} E_{U_{i} \mid \mathbf{y}_{i}, \boldsymbol{\theta}}\left[\delta_{i}^{3} \mu_{3}\left(U_{i}\right)\right]+\frac{\lambda^{4}}{\sigma_{b}^{8}} E_{U_{i} \mid \mathbf{y}_{i}, \boldsymbol{\theta}}\left[\delta_{i}^{4} \mu_{4}\left(U_{i}\right)\right]+3 E_{U_{i} \mid \mathbf{y}_{i},}, \boldsymbol{\theta}\left[\delta_{i}^{2}\right], \\
& {\widehat{U t^{2}}}_{i}=\left(\lambda^{2} \eta_{i}^{2}+\sigma_{b}^{2}\right) \widehat{U}_{i}+\lambda^{2} E_{U_{i} \mid \mathbf{y}_{i}, \boldsymbol{\theta}}\left[U_{i} \Lambda_{i}\right]+\lambda \eta_{i} E_{U_{i} \mid \mathbf{y}_{i}, \boldsymbol{\theta}}\left[U_{i} \mu_{1}\left(U_{i}\right)\right] \\
& \widehat{U t b}_{i}=\eta_{i} E_{U_{i} \mid \mathbf{y}_{i}, \boldsymbol{\theta}}\left[U_{i} \widehat{T}\left(U_{i}\right)\right]+\frac{\lambda}{\sigma_{b}^{2}} E_{U_{i} \mid \mathbf{y}_{i}, \boldsymbol{\theta}}\left[U_{i} \delta_{i}\left(\widehat{T^{2}}\left(U_{i}\right)-\lambda \eta_{i} \widehat{T}\left(U_{i}\right)\right)\right],
\end{aligned}
$$

Ferreira, C. S. 


$$
\begin{aligned}
& \widehat{U t^{2} b_{i}}=\eta_{i} E_{U_{i} \mid \mathbf{y}_{i},} \boldsymbol{\theta}\left[U_{i} \widehat{T^{2}}\left(U_{i}\right)\right]+\frac{\lambda}{\sigma_{b}^{2}} E_{U_{i} \mid \mathbf{y}_{i},} \boldsymbol{\theta}\left[U_{i} \delta_{i}\left(\widehat{T^{3}}\left(U_{i}\right)-\lambda \eta_{i} \widehat{T^{2}}\left(U_{i}\right)\right)\right], \\
& {\widehat{U t b^{2}}}_{i}=\eta_{i}^{2} E_{U_{i} \mid \mathbf{y}_{i},} \boldsymbol{\theta}\left[U_{i} \widehat{T}\left(U_{i}\right)\right]+\frac{2 \lambda \eta_{i}}{\sigma_{b}^{2}} E_{U_{i} \mid \mathbf{y}_{i}, \boldsymbol{\theta}}\left[U_{i} \delta_{i}\left(\widehat{T^{2}}\left(U_{i}\right)-\lambda \eta_{i} \widehat{T}\left(U_{i}\right)\right)\right] \\
& +\frac{\lambda^{2}}{\sigma_{b}^{4}} E_{U_{i} \mid \mathbf{y}_{i}, \boldsymbol{\theta}}\left[U_{i} \delta_{i}^{2}\left(\widehat{T^{3}}\left(U_{i}\right)-2 \lambda \eta_{i} \widehat{T^{2}}\left(U_{i}\right)+\lambda^{2} \eta_{i}^{2} \widehat{T}\left(U_{i}\right)\right)\right]+E_{U_{i} \mid \mathbf{y}_{i}, \boldsymbol{\theta}}\left[U_{i} \delta_{i} \widehat{T}\left(U_{i}\right)\right], \\
& \widehat{U t^{2} b^{2}}{ }_{i}=\eta_{i}^{2} E_{U_{i} \mid \mathbf{y}_{i}, \boldsymbol{\theta}}\left[U_{i} \widehat{T^{2}}\left(U_{i}\right)\right]+\frac{2 \lambda \eta_{i}}{\sigma_{b}^{2}} E_{U_{i} \mid \mathbf{y}_{i}, \boldsymbol{\theta}}\left[U_{i} \delta_{i}\left(\widehat{T^{3}}\left(U_{i}\right)-\lambda \eta_{i} \widehat{T^{2}}\left(U_{i}\right)\right)\right] \\
& +\frac{\lambda^{2}}{\sigma_{b}^{4}} E_{U_{i} \mid \mathbf{y}_{i}}, \boldsymbol{\theta}\left[U_{i} \delta_{i}^{2}\left(\widehat{T^{4}}\left(U_{i}\right)-2 \lambda \eta_{i} \widehat{T^{3}}\left(U_{i}\right)+\lambda^{2} \eta_{i}^{2} \widehat{T^{2}}\left(U_{i}\right)\right)\right]+E_{U_{i} \mid \mathbf{y}_{i}}, \boldsymbol{\theta}\left[U_{i} \delta_{i} \widehat{T^{2}}\left(U_{i}\right)\right] \\
& \widehat{t^{2} b^{2}}{ }_{i}=\eta_{i}^{2} E_{U_{i} \mid \mathbf{y}_{i}, \boldsymbol{\theta}}\left[\widehat{T^{2}}\left(U_{i}\right)\right]+\frac{2 \lambda \eta_{i}}{\sigma_{b}^{2}} E_{U_{i} \mid \mathbf{y}_{i}, \boldsymbol{\theta}}\left[\delta_{i}\left(\widehat{T^{3}}\left(U_{i}\right)-\lambda \eta_{i} \widehat{T^{2}}\left(U_{i}\right)\right)\right] \\
& +\frac{\lambda^{2}}{\sigma_{b}^{4}} E_{U_{i} \mid \mathbf{y}_{i}, \boldsymbol{\theta}}\left[\delta_{i}^{2}\left(\widehat{T^{4}}\left(U_{i}\right)-2 \lambda \eta_{i} \widehat{T^{3}}\left(U_{i}\right)+\lambda^{2} \eta_{i}^{2} \widehat{T^{2}}\left(U_{i}\right)\right)\right]+E_{U_{i} \mid \mathbf{y}_{i}, \boldsymbol{\theta}}\left[\delta_{i} \widehat{T^{2}}\left(U_{i}\right)\right], \\
& \widehat{U t b^{3}}{ }_{i}=\eta_{i}^{3} E_{U_{i} \mid \mathbf{y}_{i},} \boldsymbol{\theta}\left[U_{i} \widehat{T}\left(U_{i}\right)\right]+\frac{3 \lambda \eta_{i}^{2}}{\sigma_{b}^{2}} E_{U_{i} \mid \mathbf{y}_{i},} \boldsymbol{\theta}\left[U_{i} \delta_{i}\left(\widehat{T^{2}}\left(U_{i}\right)-\lambda \eta_{i} \widehat{T}\left(U_{i}\right)\right)\right] \\
& \left.+\frac{3 \lambda^{2} \eta_{i}}{\sigma_{b}^{4}} E_{U_{i} \mid \mathbf{y}_{i}, \boldsymbol{\theta}}\left[U_{i} \delta_{i}^{2} \widehat{T^{3}}\left(U_{i}\right)-2 \lambda \eta_{i} \widehat{T^{2}}\left(U_{i}\right)+\lambda^{2} \eta_{i}^{2} \widehat{T}\left(U_{i}\right)\right)\right] \\
& \left.+\frac{\lambda^{3}}{\sigma_{b}^{6}} E_{U_{i} \mid \mathbf{y}_{i}}, \boldsymbol{\theta}\left[U_{i} \delta_{i}^{3} \widehat{T^{4}}\left(U_{i}\right)-3 \lambda \eta_{i} \widehat{T^{3}}\left(U_{i}\right)+3 \lambda^{2} \eta_{i}^{2} \widehat{T^{2}}\left(U_{i}\right)\right)-\lambda^{3} \eta_{i}^{3} \widehat{T}\left(U_{i}\right)\right] \\
& +\frac{3 \lambda}{\sigma_{b}^{2}} E_{U_{i} \mid \mathbf{y}_{i}, \boldsymbol{\theta}}\left[U_{i} \delta_{i}^{2}\left(\widehat{T^{2}}\left(U_{i}\right)-\lambda \eta_{i} \widehat{T}\left(U_{i}\right)\right)\right]+3 \eta_{i} E_{U_{i} \mid \mathbf{y}_{i}, \boldsymbol{\theta}}\left[U_{i} \delta_{i} \widehat{T}\left(U_{i}\right)\right], \\
& {\widehat{t b^{3}}}_{i}=\eta_{i}^{3} E_{U_{i} \mid \mathbf{y}_{i}, \boldsymbol{\theta}}\left[\widehat{T}\left(U_{i}\right)\right]+\frac{3 \lambda \eta_{i}^{2}}{\sigma_{b}^{2}} E_{U_{i} \mid \mathbf{y}_{i},} \boldsymbol{\theta}\left[\delta_{i}\left(\widehat{T^{2}}\left(U_{i}\right)-\lambda \eta_{i} \widehat{T}\left(U_{i}\right)\right)\right] \\
& +\frac{3 \lambda^{2} \eta_{i}}{\sigma_{b}^{4}} E_{U_{i} \mid \mathbf{y}_{i}, \boldsymbol{\theta}}\left[\delta_{i}^{2}\left(\widehat{T^{3}}\left(U_{i}\right)-2 \lambda \eta_{i} \widehat{T^{2}}\left(U_{i}\right)+\lambda^{2} \eta_{i}^{2} \widehat{T}\left(U_{i}\right)\right)\right] \\
& +\frac{\lambda^{3}}{\sigma_{b}^{6}} E_{U_{i} \mid \mathbf{y}_{i},} \boldsymbol{\theta}\left[\delta_{i}^{3}\left(\widehat{T^{4}}\left(U_{i}\right)-3 \lambda \eta_{i} \widehat{T^{3}}\left(U_{i}\right)+3 \lambda^{2} \eta_{i}^{2} \widehat{T^{2}}\left(U_{i}\right)\right)-\lambda^{3} \eta_{i}^{3} \widehat{T}\left(U_{i}\right)\right] \\
& +\frac{3 \lambda}{\sigma_{b}^{2}} E_{U_{i} \mid \mathbf{y}_{i}, \boldsymbol{\theta}}\left[\delta_{i}^{2}\left(\widehat{T^{2}}\left(U_{i}\right)-\lambda \eta_{i} \widehat{T}\left(U_{i}\right)\right)\right]+3 \eta_{i} E_{U_{i} \mid \mathbf{y}_{i}, \boldsymbol{\theta}}\left[\delta_{i} \widehat{T}\left(U_{i}\right)\right], \\
& {\widehat{t^{3} b_{i}}}_{i}=\eta_{i} E_{U_{i} \mid \mathbf{y}_{i}} \boldsymbol{\theta}\left[\widehat{T^{3}}\left(U_{i}\right)\right]+\frac{\lambda}{\sigma_{b}^{2}} E_{U_{i} \mid \mathbf{y}_{i}, \boldsymbol{\theta}}\left[\delta_{i}\left(\widehat{T^{4}}\left(U_{i}\right)-\lambda \eta_{i} \widehat{T^{3}}\left(U_{i}\right)\right)\right] \mathrm{e}
\end{aligned}
$$




$$
{\widehat{t^{4}}}_{i}=E_{U_{i} \mid \mathbf{y}_{i}, \boldsymbol{\theta}}\left[\widehat{T^{4}}\left(U_{i}\right)\right]
$$

\subsection{Diagnóstico no Modelo Linear Misto Robusto}

Nesta seção serão calculadas a matriz hessiana $\left(\ddot{Q}_{\boldsymbol{\theta}}(\widehat{\boldsymbol{\theta}})\right)$ e as matrizes $\boldsymbol{\Delta}$ para análise de diagnóstico, sob o enfoque de Zhu e Lee (2001). As perturbações consideradas são ponderações de casos, perturbação no parâmetro de escala $\sigma_{b}^{2}$ (efeito aleatório), perturbação no parâmetro de escala $\sigma_{e}^{2}$ (erro), perturbação no parâmetro de assimetria $\lambda$, perturbação em uma variável explicativa e perturbação na variável resposta.

\subsubsection{A matriz hessiana}

Para obter as medidas de diagnósticos baseadas na metodologia de Zhu e Lee (2001), é necessário calcular $\ddot{Q}_{\boldsymbol{\theta}}(\widehat{\boldsymbol{\theta}})=\left.\frac{\partial^{2} Q(\boldsymbol{\theta} \mid \widehat{\boldsymbol{\theta}})}{\partial \boldsymbol{\theta} \partial \boldsymbol{\theta}^{\top}}\right|_{\boldsymbol{\theta}=\widehat{\boldsymbol{\theta}}}$, onde $\boldsymbol{\theta}=\left(\boldsymbol{\beta}^{\top}, \sigma_{e}^{2}, \sigma_{b}^{2}, \lambda\right)^{\top}$. Segue de (5.18) que $\ddot{Q}_{\boldsymbol{\theta}}(\widehat{\boldsymbol{\theta}})$ tem elementos dados por

$$
\begin{aligned}
& \frac{\partial^{2} Q(\boldsymbol{\theta} \mid \widehat{\boldsymbol{\theta}})}{\partial \boldsymbol{\beta} \partial \boldsymbol{\beta}^{\top}}=-\frac{1}{\sigma_{e}^{2}} \mathbf{X}^{\top} \mathbf{D}\left(\widehat{\mathbf{U}}^{*}\right) \mathbf{X} \\
& \frac{\partial^{2} Q(\boldsymbol{\theta} \mid \widehat{\boldsymbol{\theta}})}{\partial \boldsymbol{\beta} \partial \sigma_{e}^{2}}=-\frac{1}{\sigma_{e}^{4}} \mathbf{X}^{\top}\left[\mathbf{D}\left(\widehat{\mathbf{U}}^{*}\right)(\mathbf{Y}-\mathbf{X} \boldsymbol{\beta})-\mathbf{D}\left(\widehat{\mathbf{U b}}^{*}\right) \mathbf{Z}\right] \\
& \frac{\partial^{2} Q(\boldsymbol{\theta} \mid \widehat{\boldsymbol{\theta}})}{\partial \sigma_{e}^{2} \partial \sigma_{e}^{2}}=\frac{N}{2 \sigma_{e}^{4}}-\frac{1}{\sigma_{e}^{6}}\left[Q_{U}(\boldsymbol{\beta})-2(\mathbf{Y}-\mathbf{X} \boldsymbol{\beta})^{\top} \mathbf{D}\left(\widehat{\mathbf{U b}}^{*}\right) \mathbf{Z}+\mathbf{Z}^{\top} \mathbf{D}\left({\widehat{\mathbf{U b}^{2}}}^{*}\right) \mathbf{Z}\right] \\
& \frac{\partial^{2} Q(\boldsymbol{\theta} \mid \widehat{\boldsymbol{\theta}})}{\partial \sigma_{b}^{2} \partial \sigma_{b}^{2}}=\frac{m}{\sigma_{b}^{4}}-\frac{1}{\sigma_{b}^{6}}\left[\widehat{\mathbf{U b}^{2}}+\widehat{\mathbf{t}^{2}}-2 \lambda \widehat{\mathbf{t b}}+\lambda^{2} \widehat{\mathbf{b}^{2}}\right]^{\top} \mathbf{1}_{m}, \\
& \frac{\partial^{2} Q(\boldsymbol{\theta} \mid \widehat{\boldsymbol{\theta}})}{\partial \sigma_{b}^{2} \partial \lambda}=\frac{1}{\sigma_{b}^{4}}\left[\lambda \widehat{\mathbf{b}^{\mathbf{2}}}-\widehat{\mathbf{t b}}\right]^{\top} \mathbf{1}_{m}, \quad \frac{\partial^{2} Q(\boldsymbol{\theta} \mid \widehat{\boldsymbol{\theta}})}{\partial \lambda \partial \lambda}=-\frac{1}{\sigma_{b}^{2}}{\widehat{\mathbf{b}^{\mathbf{2}}}}^{\top} \mathbf{1}_{m}, \\
& \frac{\partial^{2} Q(\boldsymbol{\theta} \mid \widehat{\boldsymbol{\theta}})}{\partial \boldsymbol{\beta} \partial \sigma_{b}^{2}}=\frac{\partial^{2} Q(\boldsymbol{\theta} \mid \widehat{\boldsymbol{\theta}})}{\partial \boldsymbol{\beta} \partial \lambda}=\mathbf{0}: p \times 1 \mathrm{e} \\
& \frac{\partial^{2} Q(\boldsymbol{\theta} \mid \widehat{\boldsymbol{\theta}})}{\partial \sigma_{e}^{2} \partial \sigma_{b}^{2}}=\frac{\partial^{2} Q(\boldsymbol{\theta} \mid \widehat{\boldsymbol{\theta}})}{\partial \sigma_{e}^{2} \partial \lambda}=0
\end{aligned}
$$

onde $Q_{U}(\boldsymbol{\beta})=(\mathbf{Y}-\mathbf{X} \boldsymbol{\beta})^{\top} \mathbf{D}\left(\widehat{\mathbf{U}}^{*}\right)(\mathbf{Y}-\mathbf{X} \boldsymbol{\beta}), \widehat{\mathbf{t}}^{\mathbf{2}}=\left[\widehat{t}^{2}{ }_{1}, \ldots, \widehat{t}^{2}{ }_{m}\right]^{\top}, \widehat{\mathbf{U}}^{*}=\left[\widehat{u}_{1} \mathbf{1}_{n_{1}}^{\top}, \ldots, \widehat{u}_{m} \mathbf{1}_{n_{m}}^{\top}\right]^{\top}$, $\widehat{\mathbf{U b}}^{*}=\left[\widehat{u b}_{1} \mathbf{1}_{n_{1}}^{\top}, \ldots, \widehat{u b}_{m} \mathbf{1}_{n_{m}}^{\top}\right]^{\top},{\widehat{\mathbf{U b}^{2}}}^{*}=\left[\widehat{u b}^{2}{ }_{1} \mathbf{1}_{n_{1}}^{\top}, \ldots, \widehat{u b}^{2}{ }_{m} \mathbf{1}_{n_{m}}^{\top}\right]^{\top}$, $\mathbf{D}$ é a matriz diagonal e $\mathbf{1}_{m}$ é um vetor coluna $m$-dimensional de 1 's. 


\subsubsection{Perturbação de ponderação de casos}

Seja $\boldsymbol{\omega}=\left(\omega_{1}, \ldots, \omega_{m}\right)^{\top}$ um vetor de dimensão $m \times 1$ com $\boldsymbol{\omega}_{0}=(1, \ldots, 1)^{\top}$. Então, a esperança da função log-verossimilhança de dados completos perturbada (função Q perturbada), pode ser escrita como

$$
Q(\boldsymbol{\theta}, \boldsymbol{\omega} \mid \widehat{\boldsymbol{\theta}})=\sum_{i=1}^{m} \omega_{i} Q_{i}(\boldsymbol{\theta} \mid \widehat{\boldsymbol{\theta}}),
$$

onde $Q_{i}(\boldsymbol{\theta} \mid \widehat{\boldsymbol{\theta}})$ é o i-ésimo elemento da parcela $Q(\boldsymbol{\theta} \mid \widehat{\boldsymbol{\theta}})$ em (5.18). Nesse caso a matriz $\boldsymbol{\Delta}_{\boldsymbol{\omega}_{0}=}$ $\left.\frac{\partial^{2} Q(\boldsymbol{\theta}, \boldsymbol{\omega} \mid \widehat{\boldsymbol{\theta}})}{\partial \boldsymbol{\theta} \partial \boldsymbol{\omega}^{\top}}\right|_{\boldsymbol{\omega}=\boldsymbol{\omega}_{0},}=\left(\boldsymbol{\Delta}_{\boldsymbol{\beta}}^{\top}, \boldsymbol{\Delta}_{\sigma_{e}^{2}}^{\top}, \boldsymbol{\Delta}_{\sigma_{b}^{2}}^{\top}, \boldsymbol{\Delta}_{\lambda}^{\top}\right)^{\top}$ tem elementos dados por

$$
\begin{aligned}
& \boldsymbol{\Delta}_{\boldsymbol{\beta}}=\frac{1}{\sigma_{e}^{2}} \mathbf{X}^{\top}\left[\mathbf{D}(\mathbf{Y}-\mathbf{X} \boldsymbol{\beta}) \mathbf{D}^{*}\left(\widehat{\mathbf{U}}^{*}\right)-\mathbf{D}(\mathbf{Z}) \mathbf{D}^{*}\left(\widehat{\mathbf{U b}}^{*}\right)\right], \\
& \boldsymbol{\Delta}_{\sigma_{e}^{2}}=\frac{1}{2 \sigma_{e}^{4}}\left[(\mathbf{Y}-\mathbf{X} \boldsymbol{\beta})^{\top} \mathbf{D}(\mathbf{Y}-\mathbf{X} \boldsymbol{\beta}) \mathbf{D}^{*}\left(\widehat{\mathbf{U}}^{*}\right)+\mathbf{Z}^{\top} \mathbf{D}(\mathbf{Z}) \mathbf{D}^{*}\left({\widehat{\mathbf{U b}^{2}}}^{*}\right)\right. \\
& \left.-2 \mathbf{Z}^{\top} \mathbf{D}(\mathbf{Y}-\mathbf{X} \boldsymbol{\beta}) \mathbf{D}^{*}\left(\widehat{\mathbf{U b}}^{*}\right)\right]-\frac{1}{2 \sigma_{e}^{2}}\left[n_{1} n_{2} \ldots n_{m}\right], \\
& \boldsymbol{\Delta}_{\sigma_{b}^{2}}=-\frac{1}{\sigma_{b}^{2}} \mathbf{1}_{m}^{\top}+\frac{1}{2 \sigma_{b}^{4}}\left[{\widehat{\mathbf{U b}^{2}}}^{\top}+\widehat{\mathbf{t}}^{\mathbf{2}}-2 \lambda \widehat{\mathbf{t b}}+\lambda^{2}{\widehat{\mathbf{b}^{2}}}^{\top} \mathrm{e}\right. \\
& \boldsymbol{\Delta}_{\lambda}=\frac{1}{\sigma_{b}^{2}}\left[\widehat{\mathbf{t b}}-\lambda \widehat{\mathbf{b}^{2}}\right]^{\top},
\end{aligned}
$$

onde

$$
\mathbf{D}^{*}\left(\widehat{\mathbf{a}}^{*}\right)=\left(\begin{array}{cccc}
\widehat{a}_{1} \mathbf{1}_{n_{1}} & \mathbf{0}_{n_{1}} & \cdots & \mathbf{0}_{n_{1}} \\
\mathbf{0}_{n_{2}} & \widehat{a}_{2} \mathbf{1}_{n_{2}} & \cdots & \mathbf{0}_{n_{2}} \\
\vdots & \vdots & \ddots & \vdots \\
\mathbf{0}_{n_{m}} & \mathbf{0}_{n_{m}} & \cdots & \widehat{a}_{m} \mathbf{1}_{n_{m}}
\end{array}\right)
$$

é uma matriz de ordem $N \times m, \mathbf{0}_{n_{i}}$ e $\mathbf{1}_{n_{i}}$ são vetores-coluna de 0's e 1's, respectivamente, de tamanho $n_{i}$.

\subsubsection{Perturbação do parâmetro de escala do erro}

Esse esquema de perturbação é introduzido considerando na forma $\sigma_{e_{\omega_{i}}}^{2}=\sigma_{e}^{2} / \omega_{i}, \omega_{i}>0$, $i=1, \ldots, m$.

Ferreira, C. S. 
A função Q perturbada é da forma

$$
\begin{aligned}
Q(\boldsymbol{\theta}, \boldsymbol{\omega} \mid \widehat{\boldsymbol{\theta}})= & -\frac{1}{2} \sum_{i=1}^{m} n_{i} \log \frac{\sigma_{e}^{2}}{\omega_{i}}-\frac{1}{2 \sigma_{e}^{2}} \sum_{i=1}^{m} \omega_{i}\left(\widehat{U}_{i} \mathbf{Q}_{i}(\boldsymbol{\beta})-2 \widehat{U b_{i}} \mathbf{Z}_{i}^{\top}\left(\mathbf{y}_{i}-\mathbf{x}_{i}^{\top} \boldsymbol{\beta}\right)+\widehat{U b^{2}}{ }_{i} \mathbf{Z}_{i}^{\top} \mathbf{Z}_{i}\right) \\
& -m \log \sigma_{b}^{2}-\frac{1}{2 \sigma_{b}^{2}} \sum_{i=1}^{m} \widehat{u b^{2}}{ }_{i}-\frac{1}{2 \sigma_{b}^{2}} \sum_{i=1}^{m}\left(\widehat{t}^{2}{ }_{i}-2 \lambda \widehat{t b}_{i}+\lambda^{2}{\widehat{b^{2}}}_{i}\right) .
\end{aligned}
$$

Sob esse esquema de perturbação, o modelo não-perturbado segue fazendo $\boldsymbol{\omega}_{0}=(1, \ldots, 1)^{\top}$ e a matriz $\boldsymbol{\Delta}_{\boldsymbol{\omega}_{0}}$ tem elementos

$$
\begin{aligned}
& \boldsymbol{\Delta}_{\boldsymbol{\beta}}=\frac{1}{\sigma_{e}^{2}} \mathbf{X}^{\top}\left[\mathbf{D}(\mathbf{Y}-\mathbf{X} \boldsymbol{\beta}) \mathbf{D}^{*}\left(\widehat{\mathbf{U}}^{*}\right)-\mathbf{D}(\mathbf{Z}) \mathbf{D}^{*}\left(\widehat{\mathbf{U b}}^{*}\right)\right], \\
& \boldsymbol{\Delta}_{\sigma_{e}^{2}}=\frac{1}{2 \sigma_{e}^{4}}\left[(\mathbf{Y}-\mathbf{X} \boldsymbol{\beta})^{\top} \mathbf{D}(\mathbf{Y}-\mathbf{X} \boldsymbol{\beta}) \mathbf{D}^{*}\left(\widehat{\mathbf{U}}^{*}\right)+\mathbf{Z}^{\top} \mathbf{D}(\mathbf{Z}) \mathbf{D}^{*}\left({\widehat{\mathbf{U b}^{2}}}^{*}\right)\right. \\
& \left.-2 \mathbf{Z}^{\top} \mathbf{D}(\mathbf{Y}-\mathbf{X} \boldsymbol{\beta}) \mathbf{D}^{*}\left(\widehat{\mathbf{U b}}^{*}\right)\right] \text {, } \\
& \boldsymbol{\Delta}_{\sigma_{b}^{2}}=\boldsymbol{\Delta}_{\lambda}=\mathbf{0}_{m},
\end{aligned}
$$

onde $\mathbf{0}_{m}$ é um vetor linha de 0's, de tamanho $m$.

\subsubsection{Perturbação do parâmetro de escala do efeito aleatório}

Esse esquema de perturbação é introduzido considerando na forma $\sigma_{b_{\omega_{i}}}^{2}=\sigma_{b}^{2} / \omega_{i}, \omega_{i}>0$, $i=1, \ldots, m$.

A função $Q$ perturbada é da forma

$$
\begin{aligned}
Q(\boldsymbol{\theta}, \boldsymbol{\omega} \mid \widehat{\boldsymbol{\theta}})= & -\frac{N}{2} \log \sigma_{e}^{2}-\frac{1}{2 \sigma_{e}^{2}} \sum_{i=1}^{m}\left(\widehat{U}_{i} \mathbf{Q}_{i}(\boldsymbol{\beta})-2 \widehat{U b_{i}} \mathbf{Z}_{i}^{\top}\left(\mathbf{y}_{i}-\mathbf{x}_{i}^{\top} \boldsymbol{\beta}\right)+{\widehat{U b^{2}}}_{i} \mathbf{Z}_{i}^{\top} \mathbf{Z}_{i}\right) \\
& -\sum_{i=1}^{m} \log \frac{\sigma_{b}^{2}}{\omega_{i}}-\frac{1}{2 \sigma_{b}^{2}} \sum_{i=1}^{m} \widehat{\omega}_{i}{\widehat{U b^{2}}}_{i}-\frac{1}{2 \sigma_{b}^{2}} \sum_{i=1}^{m} \omega_{i}\left(\widehat{t}^{2}{ }_{i}-2 \lambda \widehat{t b}_{i}+\lambda^{2}{\widehat{b^{2}}}_{i}\right) .
\end{aligned}
$$

Sob esse esquema de perturbação, o modelo não perturbado segue considerando $\boldsymbol{\omega}_{0}=$ $(1, \ldots, 1)^{\top}$ e a matriz $\boldsymbol{\Delta}_{\boldsymbol{\omega}_{0}}$ tem elementos

$$
\begin{aligned}
\boldsymbol{\Delta}_{\boldsymbol{\beta}} & =\mathbf{0}_{m p} \text { e } \boldsymbol{\Delta}_{\sigma_{e}^{2}}=\mathbf{0}_{m}, \\
\boldsymbol{\Delta}_{\sigma_{b}^{2}} & =\frac{1}{2 \sigma_{b}^{4}}\left[\widehat{\mathbf{U b}^{2}}+\widehat{\mathbf{t}^{\mathbf{2}}}-2 \lambda \widehat{\mathbf{t b}}+\lambda^{2} \widehat{\mathbf{b}^{\mathbf{2}}}\right]^{\top} \text { e } \boldsymbol{\Delta}_{\lambda}=\frac{1}{\sigma_{b}^{2}}\left[\widehat{\mathbf{t b}}-\lambda \widehat{\mathbf{b}^{2}}\right]^{\top},
\end{aligned}
$$


onde $\mathbf{0}_{m p}$ é uma matriz de 0 's de ordem $m \times p$.

\subsubsection{Perturbação do parâmetro de assimetria}

Esse esquema de perturbação é introduzido considerando $\lambda_{\omega_{i}}=\omega_{i} \lambda$. Sendo $\boldsymbol{\omega}=$ $\left(\omega_{1}, \ldots, \omega_{n}\right)^{\top}$, a função Q perturbada é da forma

$$
\begin{aligned}
Q(\boldsymbol{\theta}, \boldsymbol{\omega} \mid \widehat{\boldsymbol{\theta}})= & -\frac{N}{2} \log \sigma_{e}^{2}-\frac{1}{2 \sigma_{e}^{2}} \sum_{i=1}^{m}\left(\widehat{U}_{i} \mathbf{Q}_{i}(\boldsymbol{\beta})-2 \widehat{U b_{i}} \mathbf{Z}_{i}^{\top}\left(\mathbf{y}_{i}-\mathbf{x}_{i}^{\top} \boldsymbol{\beta}\right)+\widehat{U b^{2}}{ }_{i} \mathbf{Z}_{i}^{\top} \mathbf{Z}_{i}\right) \\
& -m \log \sigma_{b}^{2}-\frac{1}{2 \sigma_{b}^{2}} \sum_{i=1}^{m} \widehat{U b^{2}}{ }_{i}-\frac{1}{2 \sigma_{b}^{2}} \sum_{i=1}^{m}\left(\widehat{t}^{2}{ }_{i}-2 \lambda \omega_{i} \widehat{t b}_{i}+\lambda^{2} \omega_{i} \widehat{b}^{2}{ }_{i}\right) .
\end{aligned}
$$

Sob esse esquema de perturbação, o modelo não perturbado segue considerando $\boldsymbol{\omega}_{0}=$ $(1, \ldots, 1)^{\top}$ e a matriz $\boldsymbol{\Delta} \boldsymbol{\omega}_{0}$ tem elementos

$$
\begin{aligned}
\boldsymbol{\Delta}_{\boldsymbol{\beta}} & =\mathbf{0}_{m p} \text { e } \boldsymbol{\Delta}_{\sigma_{e}^{2}}=\mathbf{0}_{m} \\
\boldsymbol{\Delta}_{\sigma_{b}^{2}} & =\frac{\lambda}{\sigma_{b}^{4}}\left[\lambda \widehat{\mathbf{b}^{\mathbf{2}}}-\widehat{\mathbf{t b}}\right]^{\top} \text { e } \boldsymbol{\Delta}_{\lambda}=\frac{1}{\sigma_{b}^{2}}\left[\widehat{\mathbf{t b}}-2 \lambda \widehat{\mathbf{b}^{2}}\right]^{\top} .
\end{aligned}
$$

\subsubsection{Perturbação em uma variável explicativa}

Nesse caso o interesse está em perturbar uma variável explicativa específica permitindo, por exemplo, detectar possíveis maus condicionamentos de alguma coluna da matriz de desenho (Belsley, 1991). Sob essa condição, tem-se a seguinte o esquema de perturbação aditiva em uma variável explicativa $\left(X_{v}\right)$

$$
\mathbf{x}_{i v_{\omega_{i}}}^{\top}=\mathbf{x}_{i}^{\top}+S_{v} \omega_{i} \mathbf{A}_{v 1 i}, \quad v \in\{1, \ldots, p\}
$$

onde $S_{v}$ é o desvio padrão da variável explicativa $\mathbf{X}_{v}$ e $\mathbf{A}_{v 1 i}$ é uma matriz de zeros de ordem $n_{i} \times p$, com a $v$-ésima coluna de 1's. Nesse caso, $\boldsymbol{\omega}_{0}=\mathbf{0}_{m}$ e a função Q perturbada é dada 
por

$$
\begin{aligned}
Q(\boldsymbol{\theta}, \boldsymbol{\omega} \mid \widehat{\boldsymbol{\theta}})= & -\frac{1}{2 \sigma_{e}^{2}} \sum_{i=1}^{m}\left[\widehat{U}_{i}\left(\mathbf{Q}_{i}(\boldsymbol{\beta})-2 \omega_{i} S_{v} \beta_{v} \mathbf{1}_{n_{i}}^{\top}\left(\mathbf{y}_{i}-\mathbf{x}_{i}^{\top} \boldsymbol{\beta}\right)+n_{i} \omega_{i}^{2} S_{v}^{2} \beta_{v}^{2}\right)\right. \\
& \left.-2 \widehat{U b_{i}} \mathbf{Z}_{i}^{\top}\left(\mathbf{y}_{i}-\mathbf{x}_{i}^{\top} \boldsymbol{\beta}-S_{v} \omega_{i} \beta_{v} \mathbf{1}_{n_{i}}\right)+\widehat{U b^{2}}{ }_{i} \mathbf{Z}_{i}^{\top} \mathbf{Z}_{i}\right] \\
& -\frac{N}{2} \log \sigma_{e}^{2}-m \log \sigma_{b}^{2}-\frac{1}{2 \sigma_{b}^{2}} \sum_{i=1}^{m}{\widehat{u b^{2}}}_{i}-\frac{1}{2 \sigma_{b}^{2}} \sum_{i=1}^{m}\left(\widehat{t}^{2}{ }_{i}-2 \lambda \widehat{t b}_{i}+\lambda^{2} \widehat{b}^{2}{ }_{i}\right) .
\end{aligned}
$$

Segue que a matriz $\boldsymbol{\Delta}_{\boldsymbol{\omega}_{0}}$ tem a forma

$$
\begin{aligned}
\boldsymbol{\Delta}_{\boldsymbol{\beta}} & =\frac{S_{v}}{\sigma_{e}^{2}}\left[\mathbf{A}_{v 1}^{\top} \mathbf{D}(\mathbf{Y}-\mathbf{X} \boldsymbol{\beta}) \mathbf{D}^{*}\left(\widehat{\mathbf{U}}^{*}\right)-\beta_{v} \mathbf{X}^{\top} \mathbf{D}^{*}\left(\widehat{\mathbf{U}}^{*}\right)-\mathbf{A}_{v 1}^{\top} \mathbf{D}^{*}(\mathbf{Z}) \mathbf{D}^{*}\left(\widehat{\mathbf{U b}}^{*}\right)\right], \\
\boldsymbol{\Delta}_{\sigma_{e}^{2}} & =\frac{S_{v} \beta_{v}}{\sigma_{e}^{4}}\left[-(\mathbf{Y}-\mathbf{X} \boldsymbol{\beta})^{\top} \mathbf{D}^{*}\left(\widehat{\mathbf{U}}^{*}\right)+\mathbf{Z}^{\top} \mathbf{D}^{*}\left(\widehat{\mathbf{U b}}^{*}\right)\right] \mathrm{e} \\
\boldsymbol{\Delta}_{\sigma_{b}^{2}} & =\boldsymbol{\Delta}_{\lambda}=\mathbf{0}_{m} .
\end{aligned}
$$

\subsubsection{Perturbação na variável resposta}

A perturbação em $Y$ é introduzida através de $\mathbf{y}_{i_{\omega_{i}}}=\mathbf{y}_{i}+S_{y} \omega_{i} \mathbf{1}_{n_{i}}$, onde $S_{y}$ é o desvio padrão de $\mathbf{Y}$. Neste caso $\boldsymbol{\omega}_{0}=\mathbf{0}_{m} \mathrm{e}$

$$
\begin{aligned}
Q(\boldsymbol{\theta}, \boldsymbol{\omega} \mid \widehat{\boldsymbol{\theta}})= & -\frac{1}{2 \sigma_{e}^{2}} \sum_{i=1}^{m}\left[\widehat{U}_{i}\left(\mathbf{Q}_{i}(\boldsymbol{\beta})+2 \omega_{i} S_{y} \mathbf{1}_{n_{i}}^{\top}\left(\mathbf{y}_{i}-\mathbf{x}_{i}^{\top} \boldsymbol{\beta}\right)+n_{i} \omega_{i}^{2} S_{y}^{2}\right)\right. \\
& \left.-2 \widehat{U b}_{i} \mathbf{Z}_{i}^{\top}\left(\mathbf{y}_{i}-\mathbf{x}_{i}^{\top} \boldsymbol{\beta}+S_{y} \omega_{i} \mathbf{1}_{n_{i}}\right)+{\widehat{U b^{2}}}_{i} \mathbf{Z}_{i}^{\top} \mathbf{Z}_{i}\right] \\
& -\frac{N}{2} \log \sigma_{e}^{2}-m \log \sigma_{b}^{2}-\frac{1}{2 \sigma_{b}^{2}} \sum_{i=1}^{m}{\widehat{u b^{2}}}_{i}-\frac{1}{2 \sigma_{b}^{2}} \sum_{i=1}^{m}\left(\widehat{t}^{2}{ }_{i}-2 \lambda \widehat{t b}_{i}+\lambda^{2}{\widehat{b^{2}}}_{i}\right) .
\end{aligned}
$$

Segue que a matriz $\boldsymbol{\Delta}_{\boldsymbol{\omega}_{0}}$ tem elementos

$$
\begin{aligned}
\boldsymbol{\Delta}_{\boldsymbol{\beta}} & =\frac{S_{y}}{\sigma_{e}^{2}} \mathbf{X}^{\top} \mathbf{D}^{*}\left(\widehat{\mathbf{U}}^{*}\right), \\
\boldsymbol{\Delta}_{\sigma_{e}^{2}} & =\frac{S_{y}}{\sigma_{e}^{4}}\left[(\mathbf{Y}-\mathbf{X} \boldsymbol{\beta})^{\top} \mathbf{D}^{*}\left(\widehat{\mathbf{U}}^{*}\right)-\mathbf{Z}^{\top} \mathbf{D}^{*}\left(\widehat{\mathbf{U b}}^{*}\right)\right] \mathrm{e} \\
\boldsymbol{\Delta}_{\sigma_{b}^{2}} & =\boldsymbol{\Delta}_{\lambda}=\mathbf{0}_{m} .
\end{aligned}
$$

A metodologia de alavancagem utilizando a função log-verossimilhança incompleta é complicada no modelo misto desenvolvido neste trabalho, pois o valor esperado do modelo 
(equação 5.14) envolve integrais numéricas envolvendo o parâmetro de assimetria $\lambda$.

\subsection{Estudo de Dados Artificiais}

Nesta seção será aplicada a metodologia proposta neste capítulo, simulando um modelo misto robusto t-normal assimétrico, sob a forma

$$
\mathbf{Y}_{i j}=\beta_{0}+x_{i j} \beta_{1}+w_{i} \beta_{2}+b_{i}+\epsilon_{i j}
$$

$\operatorname{com} j=1,2,3,4,5, i=1, \ldots, 100, x_{i j}=j-3, \beta_{0}=0, \beta_{1}=2, \beta_{2}=1, \epsilon_{i j} \sim \operatorname{Gt}\left(0,0.5^{2} ; 5,5\right) \mathrm{e}$ $b_{i} \sim G t A\left(0,0.5^{2}, 3 ; 5,5\right)$. O modelo será gerado a partir da representação estocástica (5.13), $H \sim \operatorname{Gama}(5 / 2,5 / 2)$. Note que $x_{i j}$ representa a covariável com valores mudando dentre indivíduos, porém são os mesmos para todos os indivíduos, enquanto $w_{i}=0$ se $i>50$, ou seja, uma covariável do nível do indivíduo (indicador de tratamento). Agora, em um exercício de identificar pontos atípicos, considere para os indivíduos $i=5,20$ e 50, onde o correspondente $b_{i}$ será substituído por um valor (fixo) $b_{i}=5$, de tal forma a transformá-los em ponto de alavanca. Assim, tem-se um modelo robusto como em 5.12, com três pontos extremos ("outliers").

Os gráficos de diagnósticos $(M(0))$ são apresentados a seguir, com pontos de corte na forma $\overline{M(0)}+c^{*} D P(M(0)), c *=2$ e 3 , onde $D P$ é o desvio padrão de $M(0)$. Os valores 2 e 3 de $c^{*}$ são para verificar um valor da constante que capte a sensibilidade do modelo a valores perturbados (extremos).

Os pontos perturbados 5,20 e 50 são detectados nos gráficos de ponderação de casos, perturbação no parâmetro de escala $\sigma_{b}^{2}$ e perturbação no parâmetro de assimetria $\lambda$. Por outro lado, a perturbação no parâmetro de escala $\sigma_{e}^{2}$ detecta como influentes os pontos 13, 55 e 100, enquanto as perturbações nas variáveis resposta e explicativa $\left(x_{i j}\right)$ detectam os pontos 23,31 e 68. 


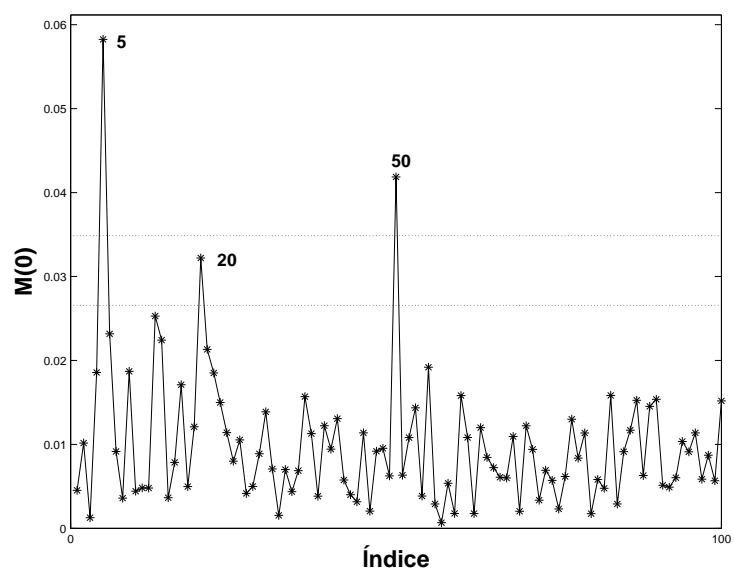

(a)

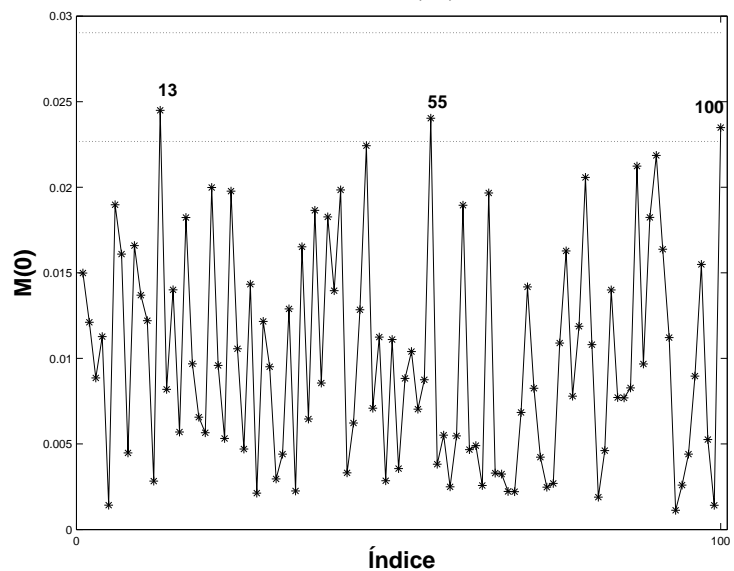

(c)

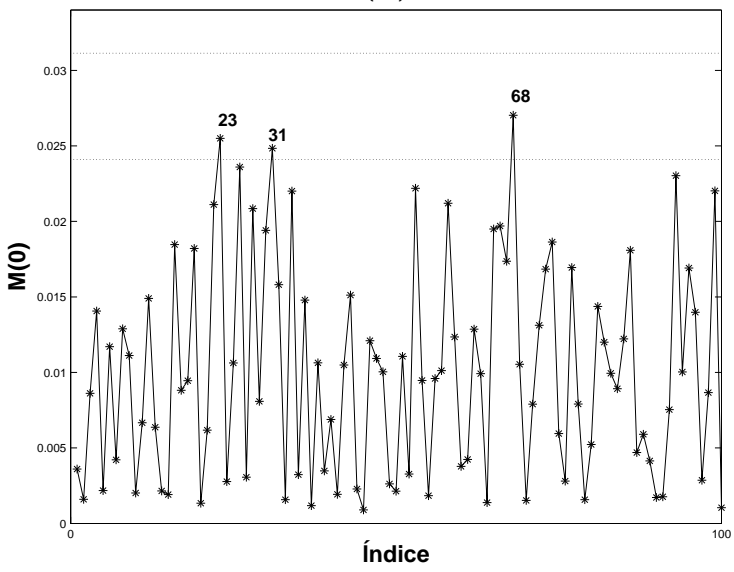

(e)

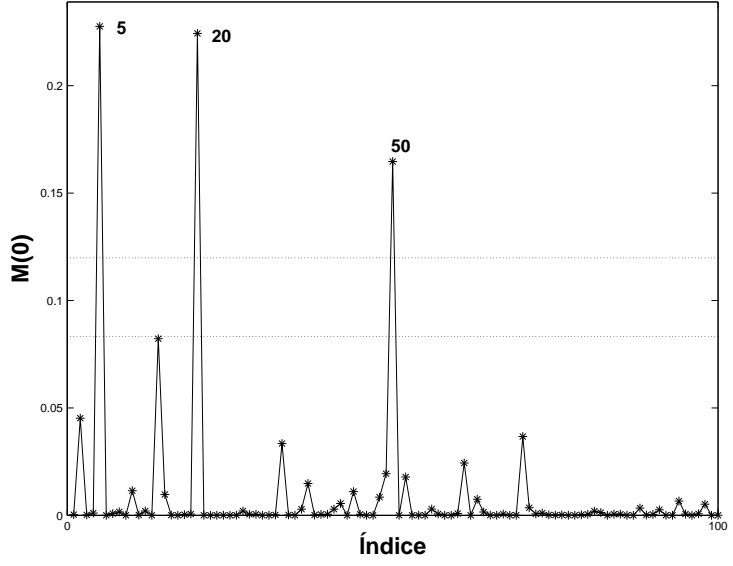

(b)

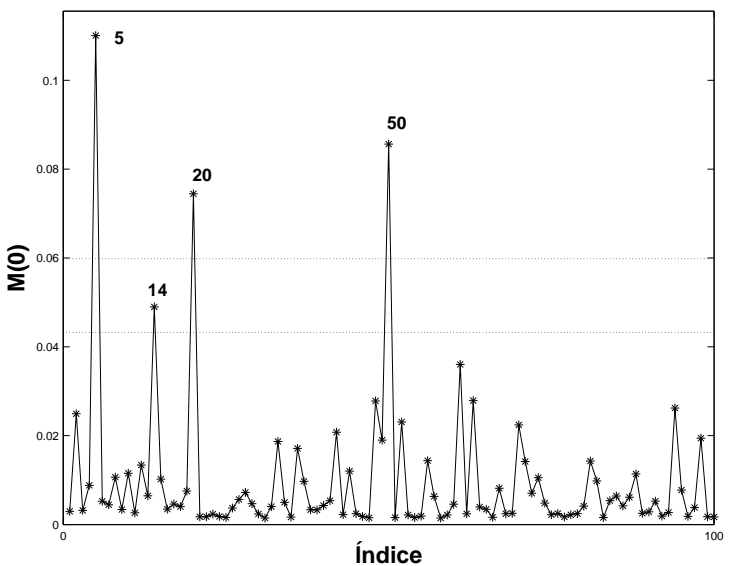

(d)

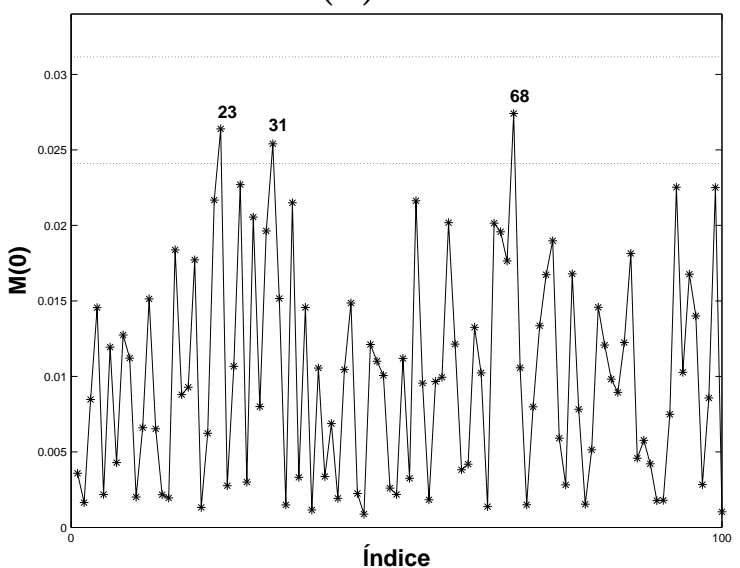

(f)

Figura 5.1: Diagnóstico no modelo de regressão misto t-normal assimétrico com dados simulados. (a) Ponderação de casos, (b) perturbação no parâmetro de assimetria, (c) perturbação no parâmetro de escala do erro, (d) perturbação no parâmetro de escala do efeito aleatório, (e) perturbação na variável resposta e (f) perturbação na variável explicativa. 


\subsection{Aplicação}

Os modelos mistos desenvolvidos neste capítulo são aplicados a um conjunto de dados longitudinais coletado como parte do conhecido estudo de colesterol de Framingham (Framingham cholesterol study), estudado previamente por Lachos et al. (2007). O conjunto de dados inclui os níveis de colesterol no tempo, idade e sexo de $m=200$ indivíduos selecionados aleatoriamente. Assim, tem-se o seguinte modelo de regressão linear misto robusto assimétrico

$$
\begin{aligned}
y_{i j} & =\beta_{0}+\beta_{1} \operatorname{sexo}_{i}+\beta_{2} \text { idade }_{i}+\beta_{3} x_{i j}+b_{i}+\varepsilon_{i j}, \\
b_{i} & \sim \operatorname{MENA}\left(0, \sigma_{b}^{2}, \lambda ; H\right), \\
\varepsilon_{i j} & \sim \operatorname{MEN}_{n_{i}}\left(\mathbf{0}_{n_{i}}, \sigma_{e}^{2} \mathbb{I}_{n_{i}} ; H\right), i=1, \ldots, 200,
\end{aligned}
$$

onde $y_{i j}$ é o nível de colesterol (mg/DL) dividido por 100, para o $j$-ésimo tempo do i-ésimo indivíduo e $x_{i j}$ é $($ tempo - 5)/10, com tempo medido em anos, a partir do início do estudo, sexo é um variável indicadora (homem $=1$, mulher $=0$ ) e idade é medida em anos. O número de repetições $j$ de cada indivíduo varia de 1 a 6 . O histograma da variável dependente nível de colesterol é apresentado na Figura 5.2, que indica uma forte assimetria (assimetria amostral de 0.69). A Tabela 5.1 apresenta as estatísticas descritivas para os níveis de colesterol e da idade dos indivíduos.

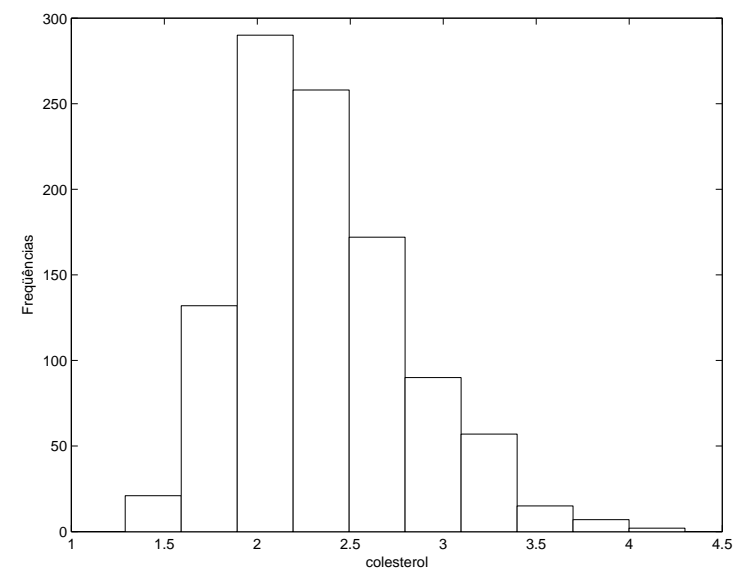

Figura 5.2: Histograma da variável colesterol (dados de Framingham).

A Tabela 5.2 apresenta as estimativas dos parâmetros do modelo misto para as distribuições normal simétrica $(\mathrm{N})$ e normal, slash, t de Student generalizada e normal con- 
Tabela 5.1: Dados de colesterol de Framingham: Estatísticas descritivas para os níveis de colesterol e de idade dos indivíduos.

\begin{tabular}{l|cccc}
\hline \hline & Mínimo & Média & Máximo & Desvio Padrão \\
\hline Nível de Colesterol (mg/DL)/100 & 1.29 & 2.34 & 4.30 & 0.46 \\
Idade (anos) & 31.0 & 42.47 & 62.0 & 7.89 \\
\hline \hline
\end{tabular}

taminada assimétricas, juntamente com os erros padrão assintóticos estimados. Nota-se que as estimativas dos coeficientes de regressão são similares em todos os modelos, porém os parâmetros de escala e de assimetria são levemente diferentes, sendo que o modelo normal assimétrico apresenta alto nível de assimetria em relação aos demais. Ainda, comparando com o modelo normal simétrico, todos os modelos assimétricos apresentam melhora no ajuste (pelo teste da razão de verossimilhanças).

O critério AIC, baseado na função log-verossimilhança, indica que os dados são melhor ajustados utilizando o modelo normal contaminado, muito embora o modelo t-normal apresente um valor muito próximo do mesmo critério. Assim, serão apresentadas as análises de diagnósticos apenas para aquele modelo.

Tabela 5.2: Estimativas dos parâmetros do modelos de regressão mistos de misturas de escala normal assimétricos para os dados de colesterol. Estimativas dos erros padrão assintóticos, através da aproximação da MI de Louis (1982), entre parênteses.

\begin{tabular}{cccccc}
\hline \hline & \multicolumn{5}{c}{ Modelos } \\
\cline { 2 - 6 } Parâmetros & $\mathrm{N}$ & $\mathrm{NA}$ & SLA & GtA & NCA \\
\hline \hline$\beta_{o}$ & $1.715(0.152)$ & $1.476(0.134)$ & $1.511(0.132)$ & $1.526(0.130)$ & $1.518(0.131)$ \\
$\beta_{1}$ & $-0.013(0.055)$ & $-0.026(0.049)$ & $-0.024(0.048)$ & $-0.031(0.047)$ & $-0.029(0.048)$ \\
$\beta_{2}$ & $0.015(0.004)$ & $0.011(0.003)$ & $0.011(0.003)$ & $0.011(0.003)$ & $0.012(0.003)$ \\
$\beta_{3}$ & $0.283(0.02)$ & $0.282(0.020)$ & $0.279(0.019)$ & $0.271(0.019)$ & $0.274(0.019)$ \\
$\sigma_{e}^{2}$ & $0.049(0.002)$ & $0.049(0.001)$ & $0.026(0.001)$ & $0.036(0.001)$ & $0.029(0.001)$ \\
$\sigma_{b}^{2}$ & $0.141(0.015)$ & $0.317(0.044)$ & $0.137(0.020)$ & $0.186(0.021)$ & $0.134(0.015)$ \\
$\lambda$ & - & $2.892(0.297)$ & $1.515(0.131)$ & $1.730(0.129)$ & $1.302(0.106)$ \\
\hline$\nu$ & - & - & 3 & 7 & 0.3 \\
$\gamma$ & - & - & - & 7 & 0.3 \\
\hline$\ell(\widehat{\boldsymbol{\theta}})$ & -186.081 & -167.632 & -154.129 & -141.606 & -140.344 \\
$\mathrm{AIC}$ & 0.369 & 0.335 & 0.309 & 0.284 & 0.282 \\
\hline \hline
\end{tabular}

Nota-se que em todos os modelos, apenas o coeficiente de regressão para a variável sexo

Ferreira, C. S. 
é não significativo (erro padrão grande em relação à estimativa do parâmetro).

Os gráficos de diagnósticos $(M(0))$ são apresentados a seguir (Figura 5.3), com pontos de corte na forma $\overline{M(0)}+c^{*} D P(M(0))$, $c *=2$ e 3 .

O indivíduo 39 exerce grande influência nas perturbações de ponderação de casos e perturbação no parâmetro de escala $\sigma_{b}^{2}$, ainda exercendo influência (considerando ponto de corte $c^{*}=2$ ) na perturbação no parâmetro de escala $\sigma_{e}^{2}$. Esse indivíduo, uma mulher, apresenta níveis de colesterol baixos (média de 1.5) e com uma idade avançada, de 59 anos, em relação aos demais.

A análise de perturbação no parâmetro de assimetria $\lambda$ indica vários pontos possivelmente exercendo influência $(2,7,26,131,160,172$ e 174), sendo a maioria acima do ponto de corte $c^{*}=3$. Esses indivíduos apresentam altos níveis de colesterol, sem caracterização específica de idade e sexo. Esses indivíduos afetam possivelmente, além da estimação do parâmetros $\lambda$, a média e a variância estimadas do modelo.

A análise de perturbação na variável resposta e na variável explicativa idade apresentam os indivíduos 43 (homem) e 156 (mulher) como influentes. Eles se caracterizam por apresentar idade avançadas, de 62 e 57 anos, respectivamente.

Ferreira, C. S. 


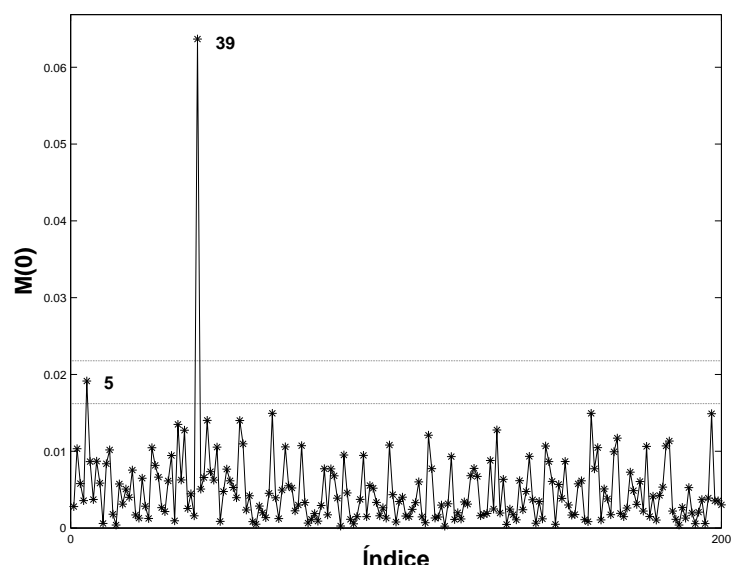

(a)

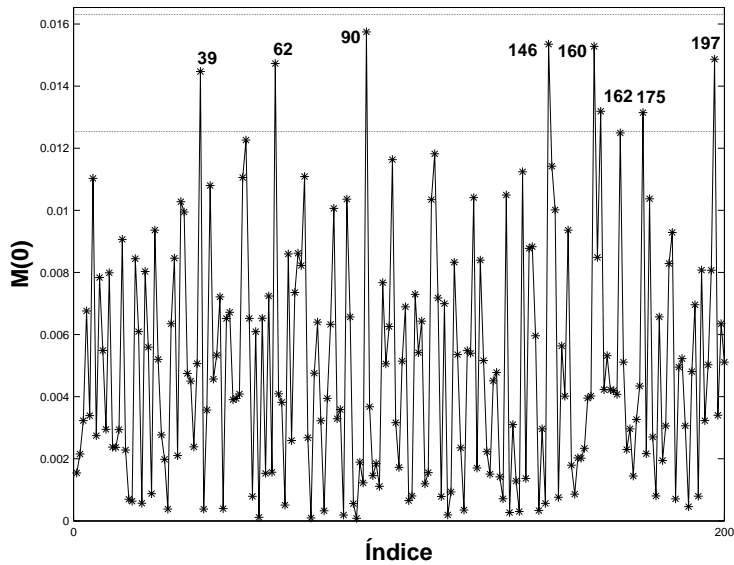

(c)

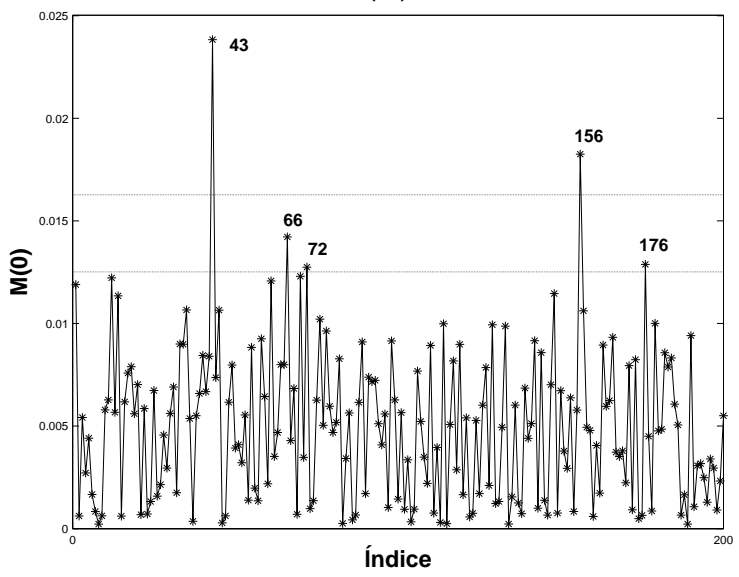

(e)

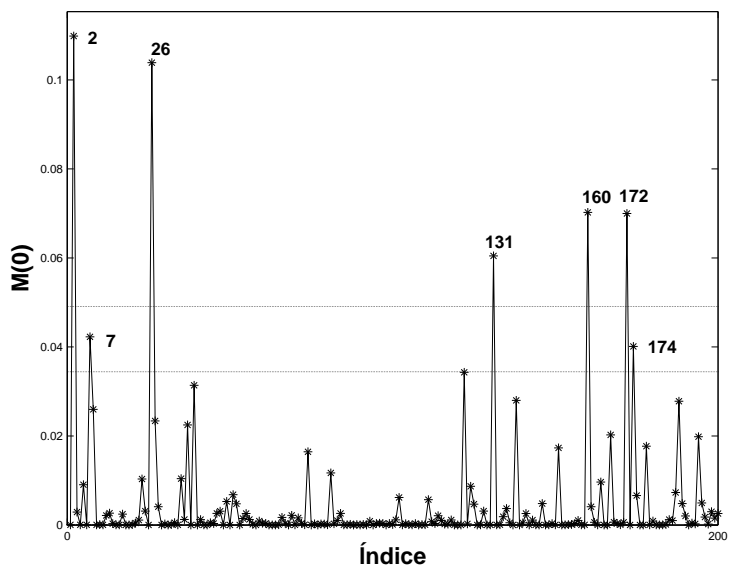

(b)

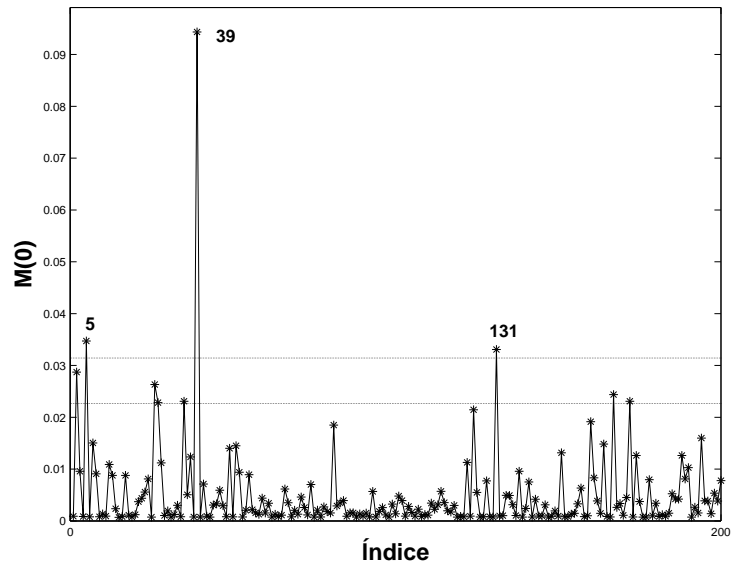

(d)

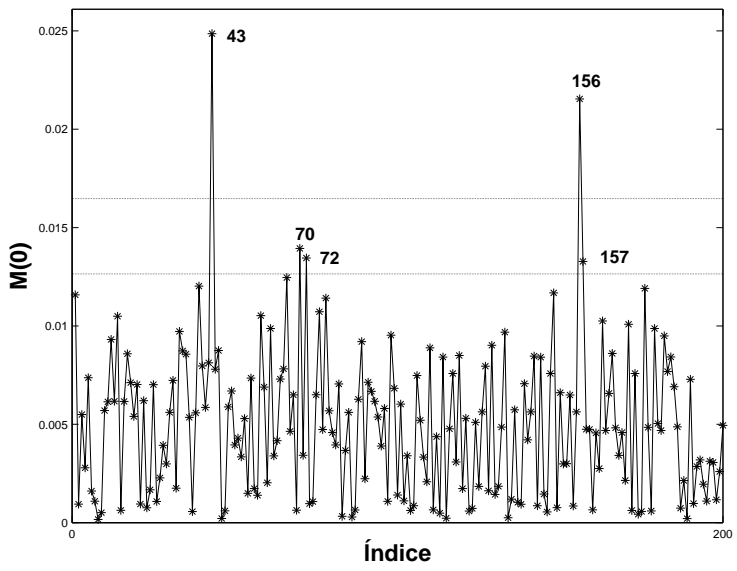

(f)

Figura 5.3: Análise de diagnóstico no modelo de regressão misto normal contaminado assimétrico para os dados de colesterol (pontos representam indivíduos). (a) Ponderação de casos, (b) perturbação no parâmetro de assimetria, (c) perturbação no parâmetro de escala do erro, (d) perturbação no parâmetro de escala do efeito aleatório, (e) perturbação na variável resposta e (f) perturbação na variável explicativa. 


\section{Considerações Finais}

\subsection{Conclusões}

Neste trabalho foi desenvolvido um estudo de inferência e diagnóstico em modelos assimétricos. A análise de diagnóstico foi baseada em técnicas gráficas (envelope simulado) e análises de influência global, local e alavancagem, através da metodologia de Zhu e Lee (2001) para dados incompletos. Para os modelos já existentes normais assimétricos (Azzalini, 1985, 1986) e t-normais assimétricos (Gómez et al., 2007), foram desenvolvidos modelos de regressão. Ainda, foram desenvolvidas duas novas classes de modelos assimétricos, a de mistura de escalas normais e a de modelos mistos de misturas de escalas normais assimétricos. Na parte de estimação dos parâmetros dos modelos, foram obtidas soluções numéricas dos estimadores de máxima verossimilhança via algoritmo EM para todos os modelos estudados. Foram utilizados os sistemas R Gui e Matlab para as programações dos processos de estimação e diagnósticos dos modelos estudados.

O Capítulo 1 descreveu a distribuição normal assimétrica introduzida por Azzalini (1985, 1986), destacando suas propriedades. Estimação, qualidade do ajuste e análise de diagnóstico foram realizadas em um conjunto de dados simulados e também em uma aplicação a dados reais, considerando um conjunto de dados da área de medicina, em um estudo sobre qualidade de vida de pacientes com câncer de mama.

O Capítulo 2 descreveu o modelo t-normal assimétrico, introduzido por Gómez et al. (2007), seus momentos, distribuição de uma forma quadrática e as matrizes de informação observada e esperada. Estimação, qualidade do ajuste e análise de diagnósticos foram realizadas em um estudo de simulação e para uma conjunto de dados reais, considerando medições de gordura corporal e quantidade de manchas na pele de atletas australianos. 
O Capítulo 3 apresentou uma nova família de distribuições assimétricas univariadas usando distribuições simétricas com mistura de escalas normais (Andrews e Mallows, 1974; Lange e Sinsheimer, 1993), englobando as distribuições normal, t de Student, slash, normal contaminada e exponencial potência assimétricas. Propriedades das distribuições foram estudadas e a implementação do algoritmo EM foi facilitada pelo fato de os passos E serem exatamente como na família de modelos normal/independente (NI), proposta em Lange e Sinsheimer (1993), obtendo uma solução analítica (no passo M) para o estimador dos parâmetros do modelo de regressão. As matrizes de informação de Fisher observadas foram calculadas para cada distribuição. Um estudo de convergência do algoritmo EM foi desenvolvido. Uma aplicação do modelo foi realizada, considerando o conjunto de dados dos atletas australianos, ajustada para cada distribuição de mistura assimétrica, construídos gráficos de envelopes e desenvolvida a análise de diagnósticos para esse conjunto de dados.

O Capítulo 4 apresentou uma nova classe de modelos lineares mistos, através da classe de distribuições de misturas de escalas normais assimétricas abordadas no Capítulo 3. O algoritmo EM foi desenvolvido, obtendo uma solução analítica para os estimadores. A Matriz de Informação Observada foi calculada via aproximação de Louis (1982), utilizando os dados incompletos. Um estudo de simulação utilizando a distribuição t-normal assimétrica foi conduzido, sob perturbação de alguns pontos e verificadas a capacidade do modelo de detectar pontos extremos. Ainda, uma aplicação utilizando os dados de colesterol de Framingham foi desenvolvida, ajustando cada distribuição de mistura, sendo desenvolvida a análise de diagnósticos para esse conjunto de dados.

A análise de diagnósticos foi realizada em todos os modelos, considerando as perturbações de ponderação de casos, perturbação no parâmetro de escala $\sigma^{2}$, perturbação no parâmetro de assimetria $\lambda$, perturbação nas variáveis Resposta e Explicativa, bem como as matrizes de alavanca calculadas. Para o modelo misto, as perturbações nos parâmetros de escala foram calculadas para os parâmetros $\sigma_{e}^{2}$ e $\sigma_{b}^{2}$.

\subsection{Perspectivas para Pesquisas Futuras}

Os modelos desenvolvidos neste trabalho permitem tanto extensões para o caso multivariado, bem como extensões da classe de misturas assimétricas a outros modelos estatísticos.

Ferreira, C. S.

IME/USP 
Os modelos de misturas assimétricos podem ser estendidos para o caso multivariado, tendo em vista que as distribuições de mistura simétricas multivariadas existem na literatura, bem como as distribuições das variáveis de mistura $U$ multivariadas e as esperanças condicionais $E\left(k^{-1}(U) \mid \mathbf{Y}\right)$ (Salgado, 2006). O modelo misto multivariado pode ser desenvolvido, considerando o efeito aleatório $\mathbf{b}_{i}$ multivariado, sendo os cálculos de estimação dos parâmetros via Algoritmo EM e da matriz de informação observada análogos aos desenvolvidos no Capítulo 4.

Em relação à extensões da classe de misturas assimétricas a outros modelos estatísticos, destaca-se alguns modelos em particular.

\section{Modelo de Calibração Comparativa Estrutural}

Suponha que se tenha à disposição $p \geq 2$ instrumentos utilizados para medir uma característica de interesse $x$ em um grupo de $n$ unidades experimentais. Seja $x_{i}$ o verdadeiro valor (desconhecido) na unidade $i$ e $y_{i j}$ o valor medido obtido com o instrumento $j$ na unidade $i$, $i=1, \ldots, n$ e $j=1, \ldots, p$. O modelo de calibração comparativa estrutural é definido pelo seguinte modelo linear

$$
\mathbf{Y}_{i}=\mathbf{a}+\mathbf{b} x_{i}+\epsilon_{i}
$$

onde $\mathbf{a}=\left(0 ; \boldsymbol{\alpha}^{\top}\right)^{\top}=\left(0 ; \alpha_{2}, \ldots, \alpha_{p}\right)^{\top}$ e $\mathbf{b}=\left(0 ; \boldsymbol{\beta}^{\top}\right)^{\top}=\left(0 ; \beta_{2}, \ldots, \beta_{p}\right)^{\top}$ são vetores $p \times 1$; $\mathbf{Y}_{i}=\left(y_{i 1}, \ldots, y_{i p}\right)^{\top}$ e $\boldsymbol{\epsilon}_{i}=\left(\epsilon_{i 1}, \epsilon_{i p}\right)^{\top}$ são vetores aleatórios $p \times 1$. É comum assumir que

$$
\boldsymbol{\epsilon}_{i} \stackrel{i i d}{\sim} N_{p}(0 ; D(\phi)) \text { e } x_{i} \stackrel{i i d}{\sim} N_{1}\left(\mu_{x} ; \sigma_{x}^{2}\right)
$$

$i=1, \ldots, n, \operatorname{com} D(\phi)=\operatorname{diag}\left(\phi_{1}, \ldots, \phi_{p}\right)$ e $\phi=\left(\phi_{1}, \ldots, \phi_{p}\right)$. Estudos inferenciais nesta classe de modelos podem ser encontrados em Galea (1995). Assim, as componentes aleatórias $\boldsymbol{\epsilon}_{i} \mathrm{e} / \mathrm{ou} x_{i}$ podem ser modeladas com distribuição de misturas de escalas normais assimétricas.

\section{Modelos de Regressão com Erros nas Variáveis com Intercepto Nulo}

Este modelo linear é definido por

$$
\begin{aligned}
& \mathbf{x}_{i}=\boldsymbol{\xi}_{i}+\boldsymbol{\delta}_{i} \\
& \mathbf{y}_{i}=\boldsymbol{\eta}_{i}+\boldsymbol{\epsilon}_{i}, \\
& \boldsymbol{\eta}_{i}=\mathbf{X}_{i} \boldsymbol{\beta}_{i}, \quad i=1, \ldots, p
\end{aligned}
$$


onde $\mathbf{x}_{i}=\left(x_{i 1}, \ldots, x_{i n_{i}}\right)^{\top}, \mathbf{y}_{i}=\left(\mathbf{y}_{i 1}^{\top}, \mathbf{y}_{i 2}^{\top}\right)^{\top}=\left(y_{1 i_{1}}, \ldots, \epsilon_{1 i_{n_{i}}}, \epsilon_{2 i_{1}}, \ldots, \epsilon_{2 i_{n_{i}}}\right), \boldsymbol{\beta}=\left(\beta_{1 i}, \beta_{2 i}\right)^{\top}$, $\boldsymbol{\xi}_{i}=\left(\xi_{i 1}, \ldots, \xi_{i n_{i}}\right)^{\top}, \boldsymbol{\delta}_{i}=\left(\delta_{i 1}, \ldots, \delta_{i n_{i}}\right)^{\top}, \boldsymbol{\epsilon}_{i}=\left(\boldsymbol{\epsilon}_{i 1}^{\top}, \boldsymbol{\epsilon}_{i 2}^{\top}\right)^{\top}=\left(\epsilon_{1 i_{1}}, \ldots, y_{1 i_{n_{i}}}, y_{2 i_{1}}, \ldots, y_{2 i_{n_{i}}}\right) \mathrm{e}$ $\mathbf{X}_{i}=\left[\begin{array}{ll}\boldsymbol{\xi} & \mathbf{0} \\ \mathbf{0} & \boldsymbol{\xi}\end{array}\right]$. Tipicamente, supõe-se que

$$
\delta_{i j} \stackrel{i i d}{\sim} N_{1}\left(0, \sigma^{2}\right), \quad \epsilon_{k i j} \stackrel{i n d}{\sim} N_{1}\left(0, \sigma_{\epsilon_{i}}^{2}\right),
$$

$\delta_{i j}$ e $\epsilon_{k i j}$ são não correlacionados e independentes de

$$
\xi_{i j} \stackrel{i i d}{\sim} N_{1}\left(\mu_{x}, \sigma_{x}^{2}\right), j=1, \ldots, n_{i}, i=1, \ldots, p, k=1,2
$$

Para mais detalhes, veja Aoki (2001). Neste caso, a extensão pode ser desenvolvida, supondo $\delta_{i j}$ ou $\epsilon_{k i j}$ com distribuição de misturas de escalas normais assimétricas. 


\section{APÊEDICE A - Modelo Normal Assimétrico}

\section{A.1 Matriz de Informação de Fisher Esperada}

Para calcular a matriz de informação de Fisher esperada $\left(I_{F}(\boldsymbol{\theta})\right)$, algumas passagens intermediárias são realizadas para facilitar as expressões da matriz. Considere $Y_{1}, \ldots, Y_{n}$ uma amostra aleatória de uma distribuição $Y \sim N A\left(\mu, \sigma^{2}, \lambda\right)$.

Sabe-se que $W_{\phi}^{\prime}\left(B_{j}\right)=-B_{j} W_{\phi}\left(B_{j}\right)-W_{\phi}^{2}\left(B_{j}\right), B_{j}=\lambda\left(Y_{j}-\mu\right) / \sigma, j=1, \ldots, n$. Dado que $Y_{j} \sim N A\left(\mu, \sigma^{2}, \lambda\right)$, tem-se que

$$
E\left[Y_{j}\right]=\mu+c \frac{\sigma \lambda}{\sqrt{1+\lambda^{2}}} \quad \text { e } \operatorname{Var}\left[Y_{j}\right]=\sigma^{2}\left(1-c^{2} \frac{\lambda^{2}}{1+\lambda^{2}}\right)
$$

sendo $c=\sqrt{\frac{2}{\pi}}$. Seja $Y_{o}=\frac{Y-\mu}{\sigma}$. Então $Y_{o} \sim N A(\lambda)$ (distribuição normal assimétrica padrão). Assim, como $B_{j}=B_{j}\left(Y_{j}, \boldsymbol{\theta}\right)=\lambda Y_{o_{j}}$, então

$$
E\left[B_{j}\left(Y_{j}, \boldsymbol{\theta}\right)\right]=c \frac{\lambda^{2}}{\sqrt{1+\lambda^{2}}} \quad \text { e } \quad E\left[\frac{Y_{j}-\mu}{\sigma}\right]^{2}=1 .
$$

Portanto, tem-se que $E\left[B_{j}\left(Y_{j}, \boldsymbol{\theta}\right)\right]^{2}=\lambda^{2}$; ainda, $E\left[Y_{j}-\mu\right]^{2}=\sigma^{2}$. Por último, tem-se que $E\left[B_{j}^{h}\left(Y_{j}, \boldsymbol{\theta}\right) W_{\phi}^{k}\left(B_{j}\left(Y_{j}, \boldsymbol{\theta}\right)\right)\right]=\lambda^{h} a_{h k}(\lambda), a_{h k}(x)=E\left(Y^{h} W_{\phi}^{k}(x Y)\right)$, com $Y \sim N A(x)$. Dado que $a_{1 k}(x)=0, k$ ímpar (integrais de produto de funções ímpares no intervalo de $(-\infty, \infty)$ ), 
tem-se que $a_{h 1}(x)=0, h$ ímpar, pois

$$
\begin{aligned}
a_{h 1}(x) & =E\left[\frac{Y^{h} \phi(x Y)}{\Phi(x Y)}\right] \\
& =\int_{-\infty}^{+\infty} \frac{y^{h} \phi(x y)}{\Phi(x y)} 2 \phi(y) \Phi(x y) d y \\
& =\int_{-\infty}^{+\infty} y^{h} \phi(x y) 2 \phi(y) d y \\
& =2 \int_{-\infty}^{+\infty} y^{h} \phi(x y) \phi(y) d y \\
& =2 \int_{-\infty}^{+\infty} g\left(y^{*}\right) \phi\left(y^{*}\right) d y^{*} \\
& =2 E\left[g\left(Y^{*}\right)\right]=0
\end{aligned}
$$

sendo $g(y)=y^{h} \phi(x y)$ uma função ímpar e $Y^{*} \sim N(0,1)$. Logo, $a_{11}(x)=0$ e $a_{31}(x)=0$.

Cálculo de $a_{01}(\cdot)$ e $a_{21}(\cdot)$

$$
\begin{aligned}
a_{01}(x) & =\frac{2}{\sqrt{2 \pi}} \int_{-\infty}^{\infty} \frac{1}{\sqrt{2 \pi}} \exp \left\{-\frac{y^{2}}{2 /\left(x^{2}+1\right)}\right\} d y \\
& =\frac{2}{\sqrt{2 \pi}} \int_{-\infty}^{\infty} \frac{1}{\sqrt{2 \pi}} \frac{\sqrt{1 /\left(x^{2}+1\right)}}{\sqrt{1 /\left(x^{2}+1\right)}} \exp \left\{-\frac{y^{2}}{2 /\left(x^{2}+1\right)}\right\} d y \\
& =\frac{2}{\sqrt{2 \pi}} \sqrt{1 /\left(x^{2}+1\right)} \cdot 1 \\
& =\sqrt{\frac{2}{\pi}}\left(x^{2}+1\right)^{-1 / 2}
\end{aligned}
$$

$\mathrm{e}$

$$
\begin{aligned}
a_{21}(x) & =\frac{2}{\sqrt{2 \pi}} \int_{-\infty}^{\infty} \frac{y^{2}}{\sqrt{2 \pi}} \exp \left\{-\frac{y^{2}}{2 /\left(x^{2}+1\right)}\right\} d y \\
& =\frac{2}{\sqrt{2 \pi}} \int_{-\infty}^{\infty} \frac{y^{2}}{\sqrt{2 \pi}} \frac{\sqrt{1 /\left(x^{2}+1\right)}}{\sqrt{1 /\left(x^{2}+1\right)}} \exp \left\{-\frac{y^{2}}{2 /\left(x^{2}+1\right)}\right\} d y \\
& =\frac{2}{\sqrt{2 \pi}}\left(x^{2}+1\right)^{-1 / 2} \cdot\left(x^{2}+1\right)^{-1} \\
& =\sqrt{\frac{2}{\pi}}\left(x^{2}+1\right)^{-3 / 2} .
\end{aligned}
$$

Cálculo de $a_{02}(\cdot), a_{12}(\cdot)$ e $a_{22}(\cdot)$

Utiliza-se a aproximação dada em Rodriguez, 2002.

Ferreira, C. S. 
Para $h$ ímpar, tem-se que

$$
\begin{aligned}
a_{h 2}(x) \approx & -\frac{2^{\frac{h+2}{2}}}{\pi^{3 / 2}}\left(\frac{h-1}{2}\right) ! x\left(1+\frac{2 x^{2}}{\pi^{2} / 4}\right)^{-\frac{h+2}{2}}\left[1+x^{2}\left(1+\frac{2 x^{2}}{\pi^{2} / 4}\right)^{-1}\right]^{-1 / 2} \\
& \times\left\{\sum_{j=0}^{\frac{h-1}{2}} c_{j} x^{j}\left(1+\frac{2 x^{2}}{\pi^{2} / 4}\right)^{-1 / 2}\right\},
\end{aligned}
$$

sendo $c_{0}=1$ e $c_{j}=\frac{2 j-1}{2 j} \cdot c_{j-1}$.

Portanto,

$$
a_{12}(\lambda) \approx-\frac{2^{3 / 2}}{\pi^{3 / 2}} 0 ! \lambda\left(1+\lambda^{2} \frac{8}{\pi^{2}}\right)^{-3 / 2}\left[1+\lambda^{2}\left(1+\lambda^{2} \frac{8}{\pi^{2}}\right)^{-1}\right]^{-1 / 2}
$$

Para $h$ par, a aproximação para $a_{h 2}(x)$ é dada por

$$
a_{h 2}(x) \approx-\frac{1}{2^{h / 2-1} \pi} \frac{h !}{(h / 2) !}\left(1+\frac{8 x^{2}}{\pi^{2}}\right)^{-\frac{h+1}{2}} .
$$

Logo, tem-se que

$$
a_{02}(\lambda) \approx \frac{2}{\pi}\left(1+\lambda^{2} \frac{8}{\pi^{2}}\right)^{-1 / 2} \quad \text { e } \quad a_{22}(\lambda) \approx \frac{2}{\pi}\left(1+\lambda^{2} \frac{8}{\pi^{2}}\right)^{-3 / 2} .
$$

\section{Matriz de Informação de Fisher Esperada}

$$
\begin{aligned}
I_{\boldsymbol{\beta} \boldsymbol{\beta}} & =\frac{1}{\sigma^{2}}\left[1+\lambda^{2} a_{02}(\lambda)\right] \boldsymbol{X}^{\top} \boldsymbol{X} \\
I_{\boldsymbol{\beta}_{\sigma^{2}}} & =\frac{n \delta}{\sigma^{3}}\left[\frac{c}{\sigma}-\frac{\left(2+\lambda^{2}\right)\left(1+\lambda^{2}\right)^{1 / 2}}{2 \sigma}\left(a_{01}(\lambda)-\lambda a_{12}(\lambda)-\lambda^{2} a_{21}(\lambda)\right)\right] \overline{\mathbf{x}}, \\
I_{\boldsymbol{\beta}_{\lambda}} & =\frac{n}{\sigma\left(1+\lambda^{2}\right)^{1 / 2}}\left[2 c \frac{\sigma \lambda^{2}}{1+\lambda^{2}}-\delta\left[\lambda a_{21}(\delta / \sigma)+a_{12}(\lambda)\right]+\frac{\sigma}{\left(1+\lambda^{2}\right)^{1 / 2}} a_{01}(\lambda)\right] \overline{\mathbf{x}}, \\
I_{\sigma^{2} \sigma^{2}} & =\frac{n}{\sigma^{4}}\left[\frac{1}{2}+\frac{\delta^{2}\left(2+\lambda^{2}\right)\left(1+\lambda^{2}\right)^{2}}{4 \sigma^{4}} a_{22}(\lambda)\right], \\
I_{\sigma^{2} \lambda} & =\frac{n \delta}{\sigma^{4}}\left[1-\frac{2+\lambda^{2}}{2} a_{22}(\lambda)\right] \mathrm{e} \\
I_{\lambda \lambda} & =\frac{n}{\sigma^{4}}\left[2 \delta^{2}+\frac{\sigma^{2}}{1+\lambda^{2}} a_{22}(\lambda)\right],
\end{aligned}
$$

onde $\delta=\sigma \lambda / \sqrt{1+\lambda^{2}}, \overline{\mathbf{x}}=\frac{1}{n}\left(n, \sum_{i=1}^{n} x_{i 1}, \cdots, \sum_{i=1}^{n} x_{i p}\right)$. 


\title{
APÊEDICE B - Modelo t-Normal Assimétrico
}

\author{
Matriz de Informação Esperada
}

De Gómez et al. (2007), segue que

$$
I_{F}(\boldsymbol{\theta})=E[\ddot{L}(\boldsymbol{\theta})]=\left(\begin{array}{ccc}
I_{\boldsymbol{\beta} \boldsymbol{\beta}} & I_{\boldsymbol{\beta}_{\sigma^{2}}} & I_{\boldsymbol{\beta} \lambda} \\
& I_{\sigma^{2} \sigma^{2}} & I_{\sigma^{2} \lambda} \\
& & I_{\lambda \lambda}
\end{array}\right)
$$

com elementos dados por

$$
\begin{aligned}
I_{\boldsymbol{\beta} \boldsymbol{\beta}} & =\left[\frac{\nu+1}{\sigma^{2}(\nu+3)}+\frac{\lambda^{2}}{\pi \sigma^{2}} c(\nu) I_{0}\right] \mathbf{X}^{\top} \mathbf{X} \\
I_{\boldsymbol{\beta}_{\sigma^{2}}} & =\frac{c(\nu)}{2 \sigma^{3}}\left[\frac{4(\nu+1)}{\nu} J_{0}-\frac{4(\nu+1)}{\nu^{2}} J_{1}-\frac{2 \lambda}{\sqrt{2 \pi}} K_{0}+\frac{2 \lambda^{3}}{\sqrt{2 \pi}} K_{1}+\frac{\lambda^{2}}{\pi} I_{1}\right] \mathbf{X}^{\top} \mathbf{1}_{n} \\
I_{\boldsymbol{\beta}_{\lambda}} & =\frac{2}{\sigma \sqrt{2 \pi}} c(\nu)\left[K_{0}-\lambda^{2} K_{1}-\frac{\lambda}{\sqrt{2 \pi}} I_{1}\right] \mathbf{x}^{\top} \mathbf{1}_{n} \\
I_{\sigma^{2} \sigma^{2}} & =\frac{n}{2 \sigma}\left[\frac{2 \nu}{\sigma^{2}(\nu+3)}+\frac{\lambda^{2}}{\sigma^{2} \pi} c(\nu) I_{2}\right] \\
I_{\lambda \lambda} & =\frac{n}{\pi} c(\nu) I_{2} \mathrm{e} \\
I_{\sigma^{2} \lambda} & =-n \frac{\lambda}{2 \pi \sigma^{2}} c(\nu) I_{2}
\end{aligned}
$$


onde $\mathbf{1}_{n}$ é um vetor de 1's de tamanho $n, c(\nu)=\frac{2 \Gamma\left(\frac{\nu+1}{2}\right)}{\sqrt{\pi \nu} \Gamma\left(\frac{\nu}{2}\right)} \mathrm{e}$

$$
\begin{aligned}
I_{i} & =\int_{-\infty}^{\infty} u^{i}\left(1+\frac{u^{2}}{\nu}\right)^{-\frac{\nu+1}{2}} \exp \left(-\lambda^{2} u^{2}\right) \frac{1}{\Phi_{1}(\lambda u)} d u, \quad i=0,1,2, \\
J_{i} & =\int_{-\infty}^{\infty} u^{2 i+1}\left(1+\frac{u^{2}}{\nu}\right)^{-\frac{\nu+2 i+3}{2}} \Phi_{1}(\lambda u) d u, \quad i=0,1, \\
K_{i} & =\int_{-\infty}^{\infty} u^{2 i}\left(1+\frac{u^{2}}{\nu}\right)^{-\frac{\nu+1}{2}} \exp \left(-\lambda^{2} u^{2} / 2\right) d u, \quad i=0,1,
\end{aligned}
$$

que devem ser calculadas numericamente, utilizando integração de Monte Carlo.

\section{B.1 Gráficos de Diagnóstico para os Dados Simulados}

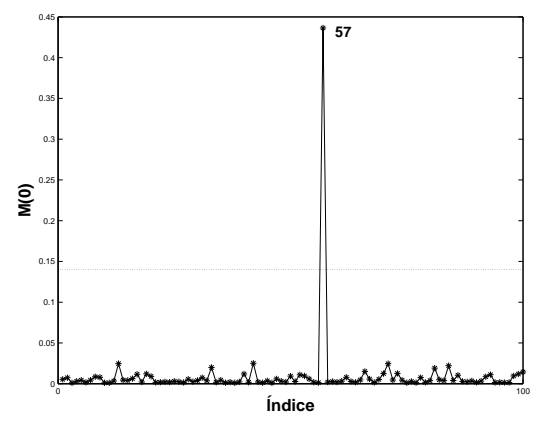

(a)

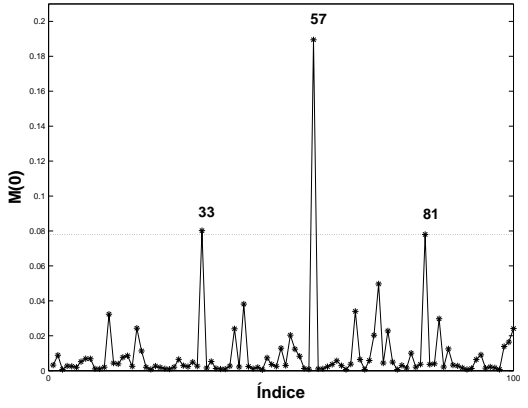

(b)

Figura B.1: Ponderação de Casos (a) perturbação $y(57)=10$ e (b) perturbação $y(33)=$ $y(81)=19$.

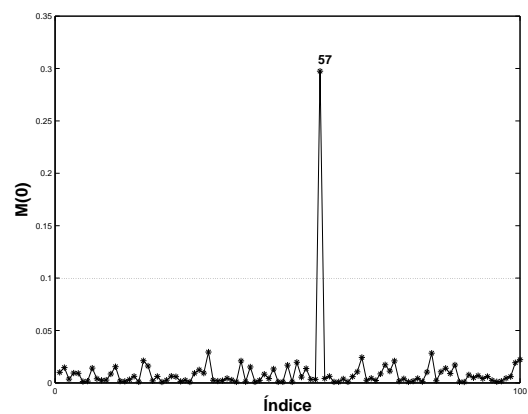

(a)

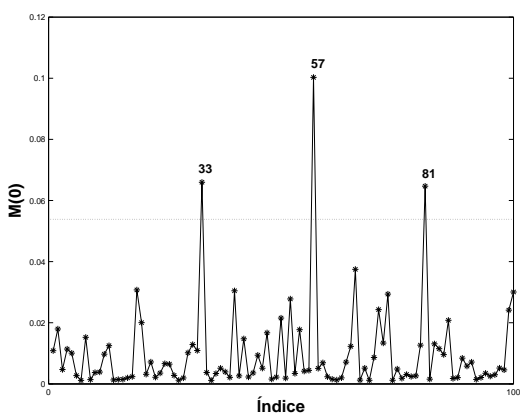

(b)

Figura B.2: Perturbação no parâmetro de escala (a) perturbação $y(57)=10$ e (b) perturbação $y(33)=y(81)=19$. 


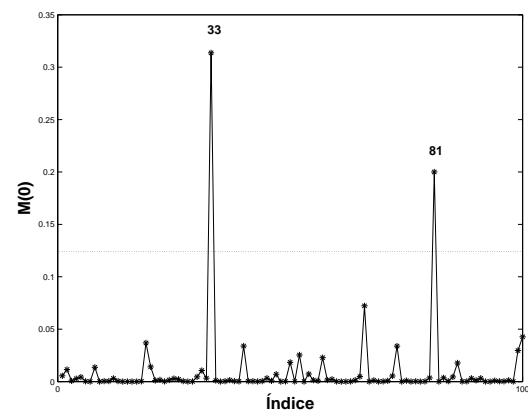

(a)

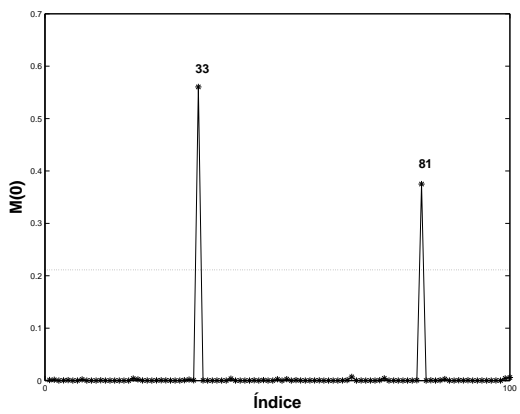

(b)

Figura B.3: Perturbação no parâmetro de assimetria (a) perturbação $y(57)=10$ e (b) perturbação $y(33)=y(81)=19$.

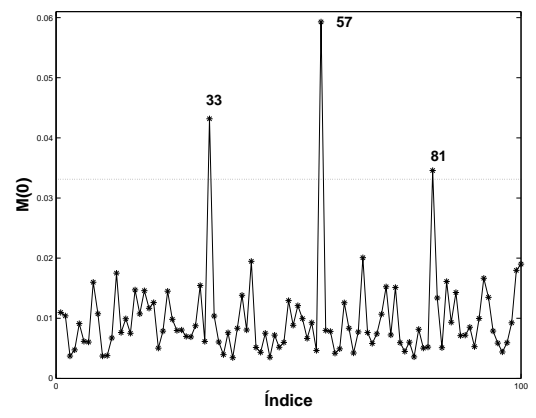

(a)

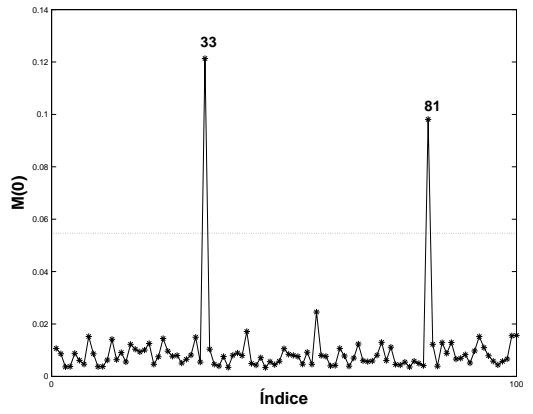

(b)

Figura B.4: Perturbação na variável resposta (a) perturbação $y(57)=10$ e (b) perturbação $y(33)=y(81)=19$.

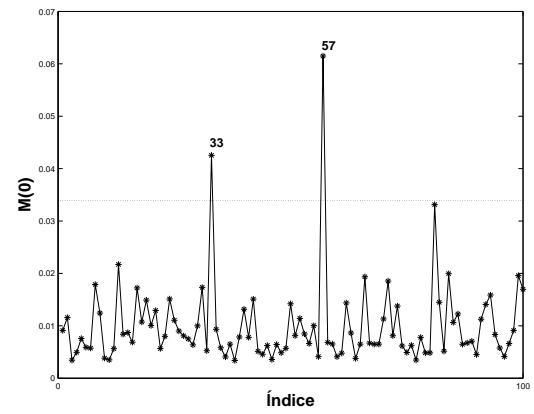

(a)

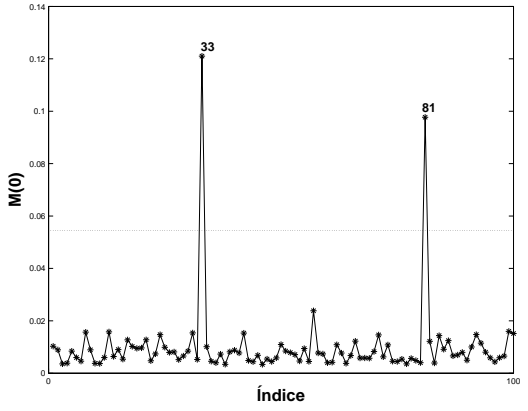

(b)

Figura B.5: Perturbação na variável explicativa (a) perturbação $y(57)=10$ e (b) perturbação $y(33)=y(81)=19$.

Ferreira, C. S. 


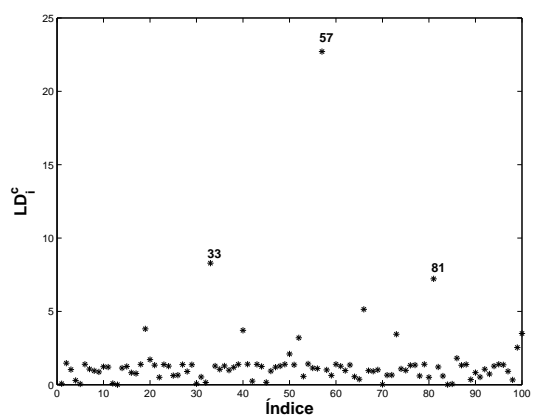

(a)

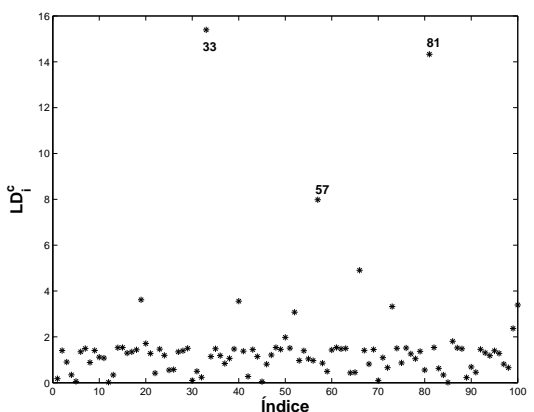

(b)

Figura B.6: Influência global $\left(L D_{i}^{c}\right)($ a) perturbação $y(57)=10$ e (b) perturbação $y(33)=$ $y(81)=19$.

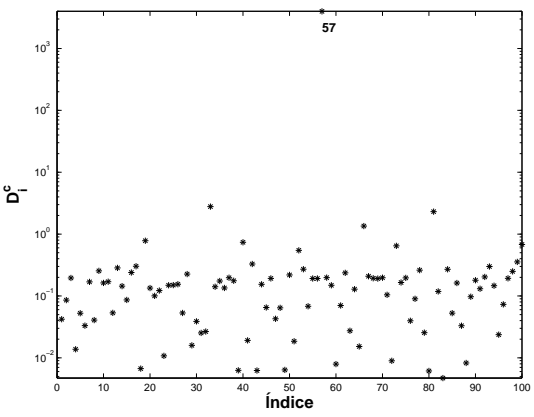

(a)

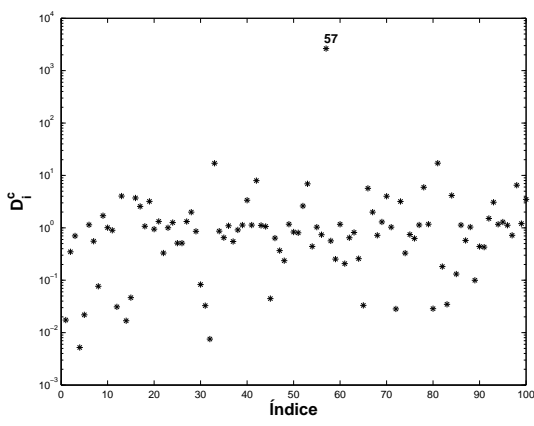

(b)

Figura B.7: Influência global $\left(D_{i}^{c}\right)$ (a) perturbação $y(57)=10$ e (b) perturbação $y(33)=$ $y(81)=19$. 


\section{APÊNDICE C - Modelo de Misturas de Escala Normal Assimétrico}

\section{C.1 Função Densidade de Probabilidade de Distribuições de Misturas de Escala Normal Assimétricas}

Seja $Y$ com distribuição na classe MENA com parâmetro de locação $\mu \in \mathbb{R}$, de escala $\sigma^{2}$ e se assimetria $\lambda \in \mathbb{R}$. Sua fdp é dada por

$$
f(y)=2 \int_{0}^{+\infty} \phi\left(y \mid \mu, \sigma^{2} \kappa(u)\right) \Phi_{1}\left(\lambda \frac{y-\mu}{\sigma}\right) d H(u),
$$

onde $U$ é uma variável aleatória positiva com fda $H(u ; \boldsymbol{\nu})$. Assim, tem-se que

$$
\begin{aligned}
f(y) & =2 \int_{0}^{+\infty} \phi\left(y \mid \mu, \sigma^{2} \kappa(u)\right) h(u ; \nu) d u \int_{-\infty}^{\lambda \frac{y-\mu}{\sigma}} \phi(t \mid 0,1) d t \\
& =2 \int_{0}^{+\infty} \int_{-\infty}^{0} \phi\left(y \mid \mu, \sigma^{2} \kappa(u)\right) \phi\left(t \mid-\lambda \frac{y-\mu}{\sigma}, 1\right) h(u ; \nu) d t d u \\
& =2 \int_{0}^{+\infty} \int_{0}^{+\infty} \phi\left(y \mid \mu, \sigma^{2} \kappa(u)\right) \phi\left(t \mid \lambda(y-\mu), \sigma^{2}\right) h(u ; \nu) d t d u
\end{aligned}
$$


pois

$$
\begin{aligned}
\int_{-\infty}^{0} \phi\left(t \mid-\lambda \frac{y-\mu}{\sigma}, 1\right) & =P(Z \leq 0), Z \sim N\left(-\lambda \frac{y-\mu}{\sigma}, 1\right) \\
& =P(-Z \geq 0) \\
& =P(T \geq 0), T \sim N\left(\lambda \frac{y-\mu}{\sigma}, 1\right) \\
& =\int_{0}^{+\infty} \phi\left(t \mid \lambda \frac{y-\mu}{\sigma}, 1\right) \\
& =\int_{0}^{+\infty} \phi\left(t \mid \lambda(y-\mu), \sigma^{2}\right) .
\end{aligned}
$$

\section{C.2 Gráficos de Diagnósticos}

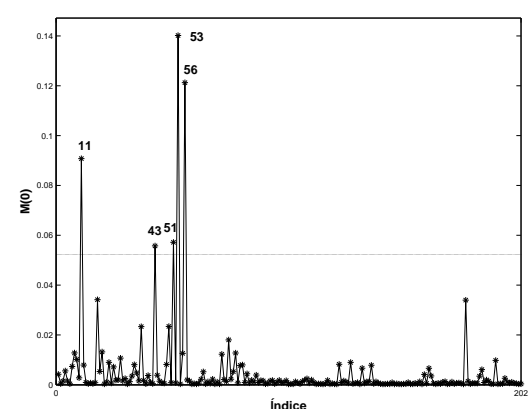

(a)

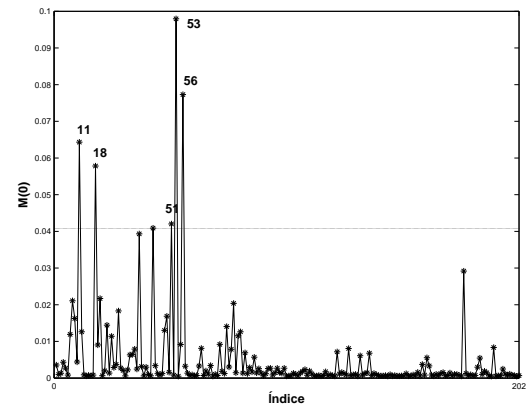

(c)

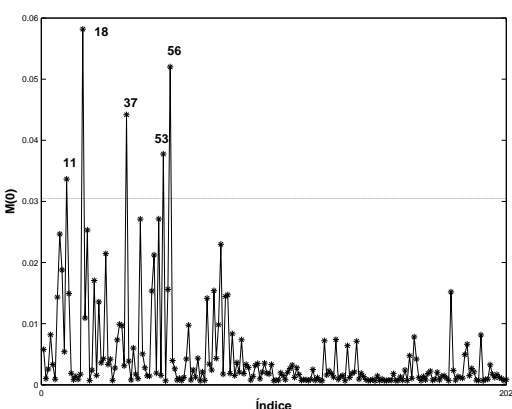

(b)

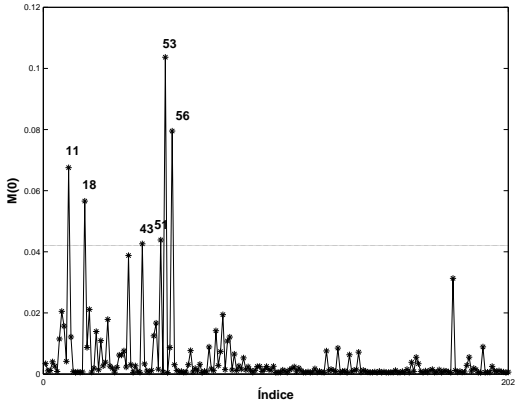

(d)

Figura C.1: Dados AIS. Perturbação de ponderação de casos nos modelos assimétricos: (a) normal (b) t de Student (c) exponencial potência e (d) slash. 


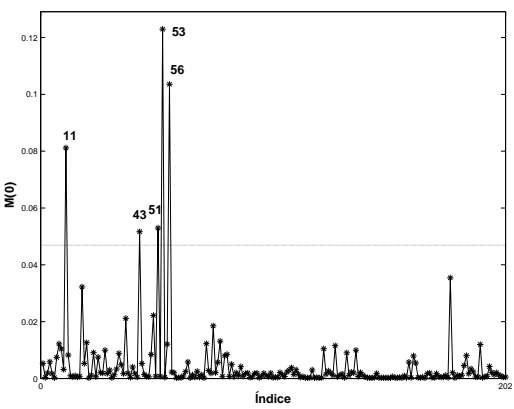

(a)

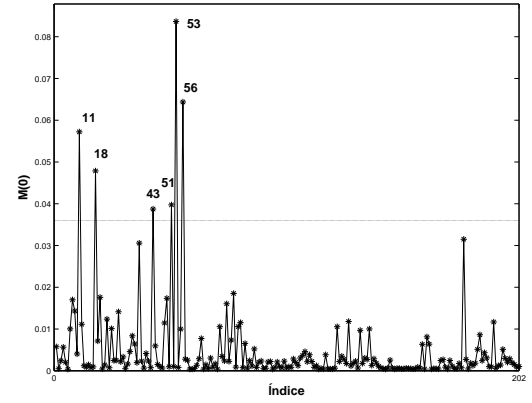

(c)

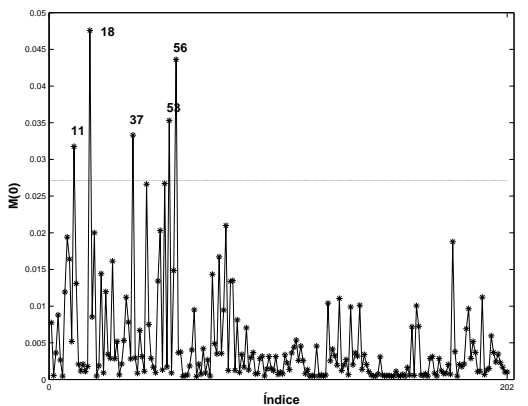

(b)

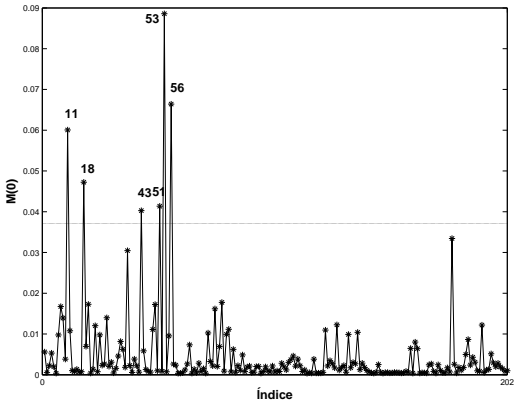

(d)

Figura C.2: Dados AIS. Perturbação no parâmetro de escala nos modelos assimétricos: (a) normal (b) t de Student (c) exponencial potência e (d) slash.

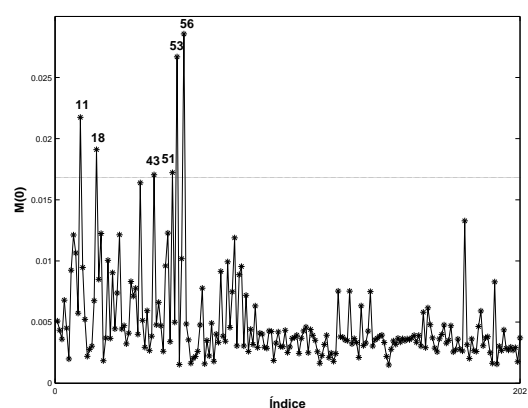

(a)

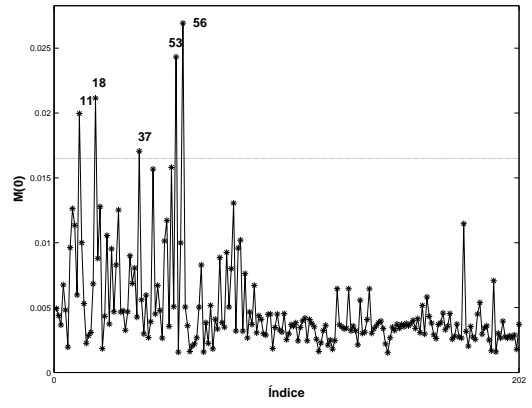

(c)

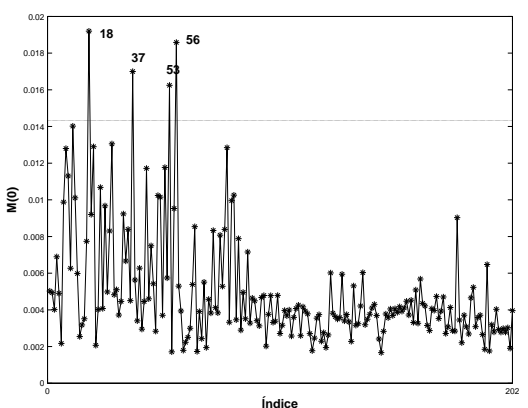

(b)

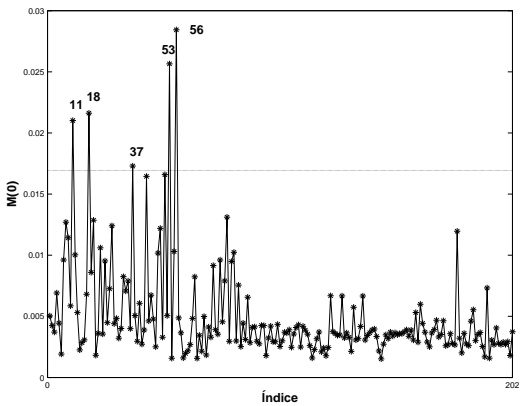

(d)

Figura C.3: Dados AIS. Perturbação na variável resposta nos modelos assimétricos: (a) normal (b) t de Student (c) exponencial potência e (d) slash.

Ferreira, C. S. 


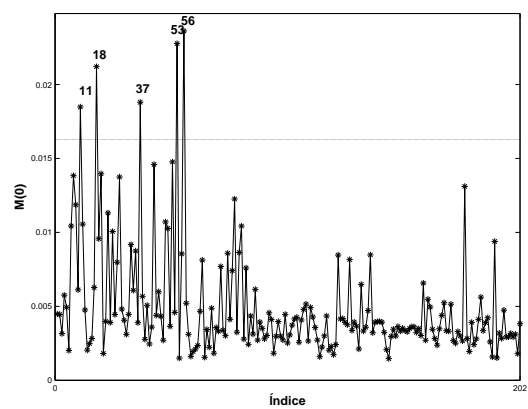

(a)

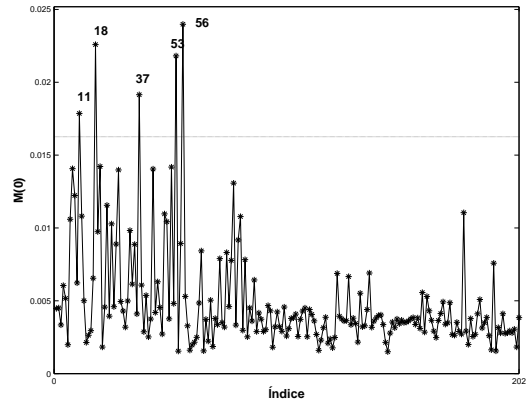

(c)

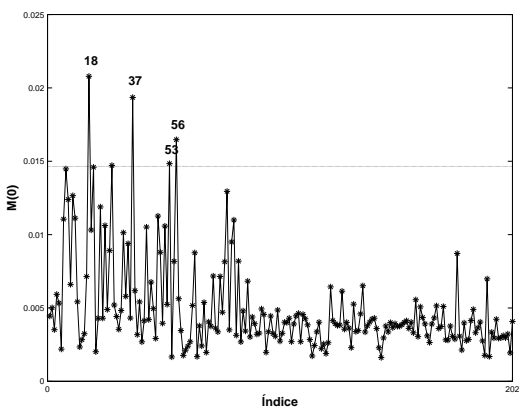

(b)

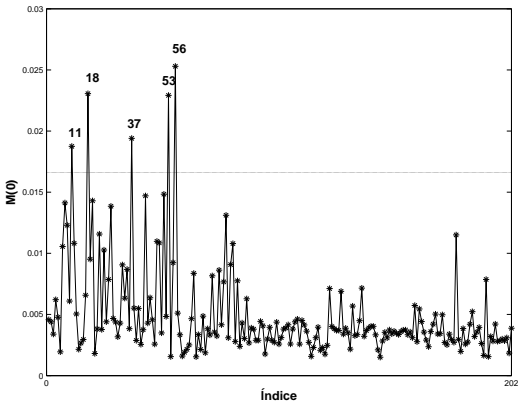

(d)

Figura C.4: Dados AIS. Perturbação em uma variável explicativa nos modelos assimétricos: (a) normal (b) t de Student (c) exponencial potência e (d) slash.

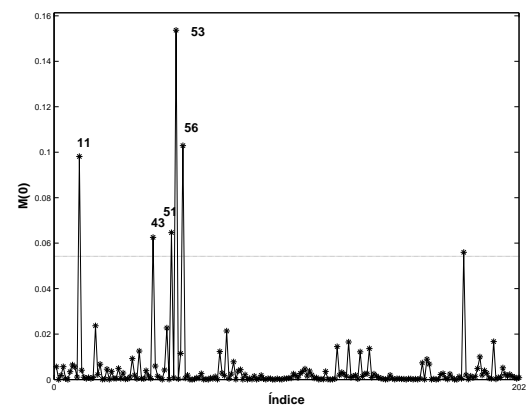

(a)

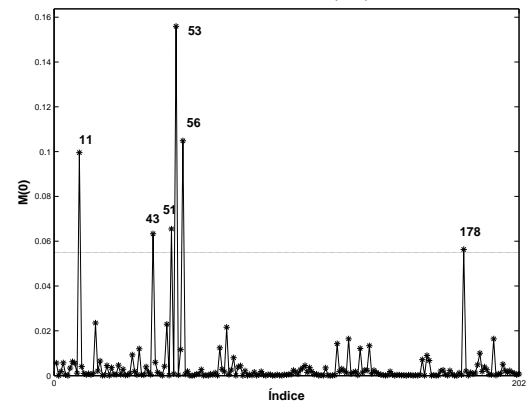

(c)

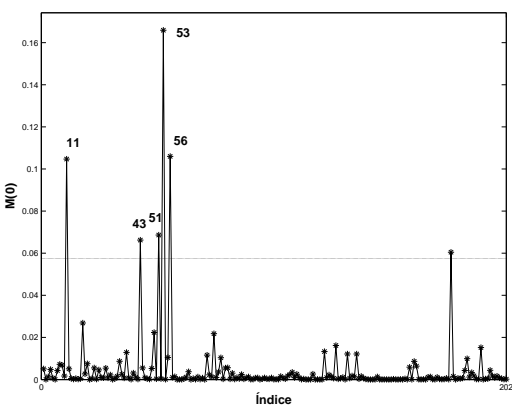

(b)

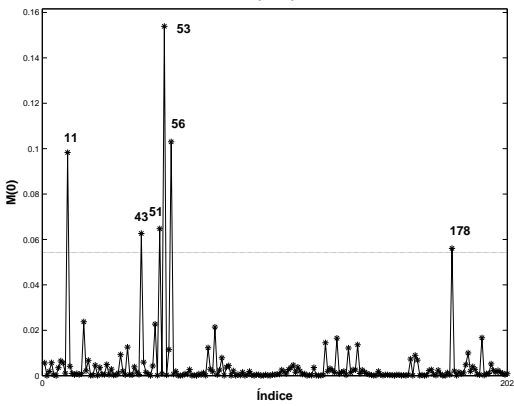

(d)

Figura C.5: Dados AIS. Perturbação no parâmetro de assimetria nos modelos assimétricos: (a) normal (b) t de Student (c) exponencial potência e (d) slash. 


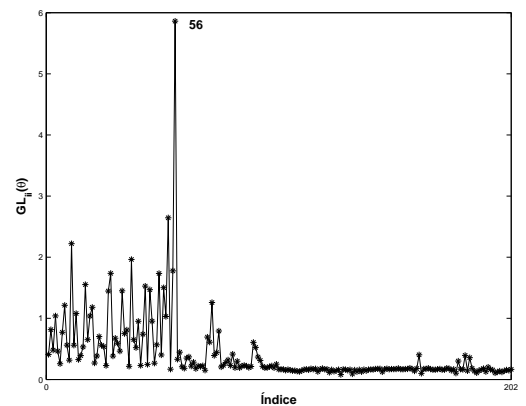

(a)

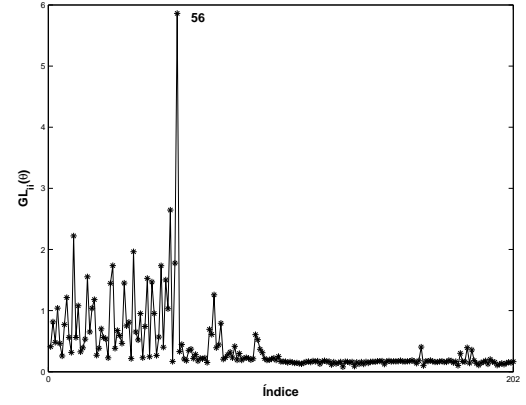

(c)

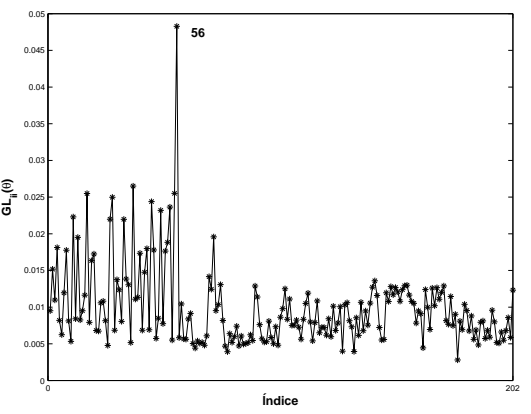

(b)

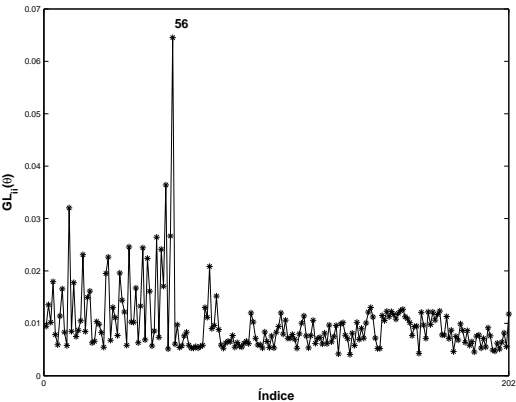

(d)

Figura C.6: Dados AIS. Alavanca nos modelos assimétricos: (a) normal (b) t de Student (c) exponencial potência e (d) slash.

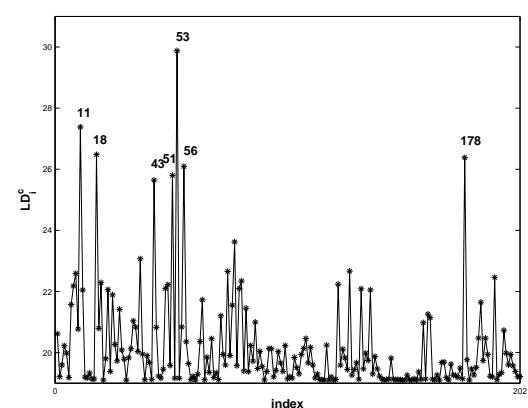

(a)

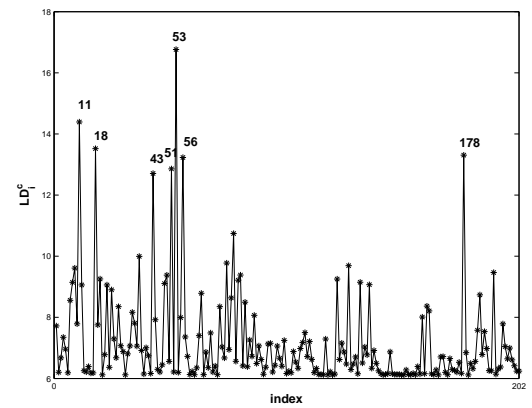

(c)

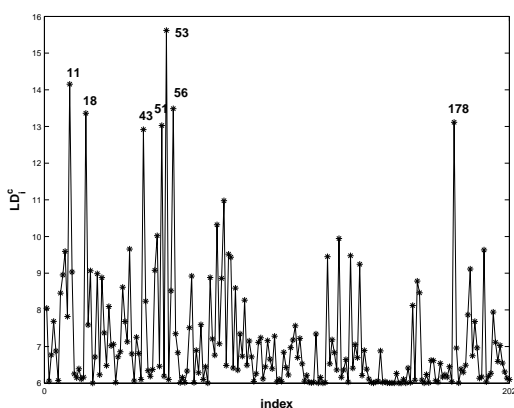

(b)

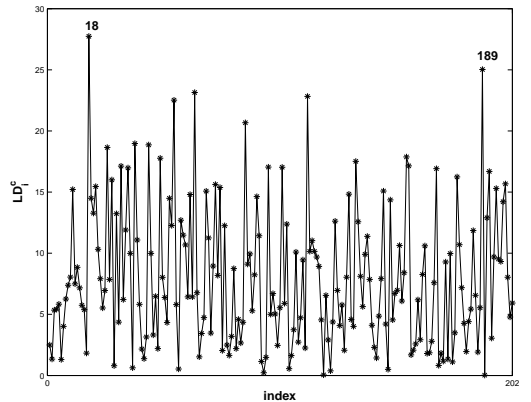

(d)

Figura C.7: Dados AIS. Influência global ( $L D i)$ nos modelos assimétricos: (a) normal (b) t de Student (c) exponencial potência e (d) slash.

Ferreira, C. S. 


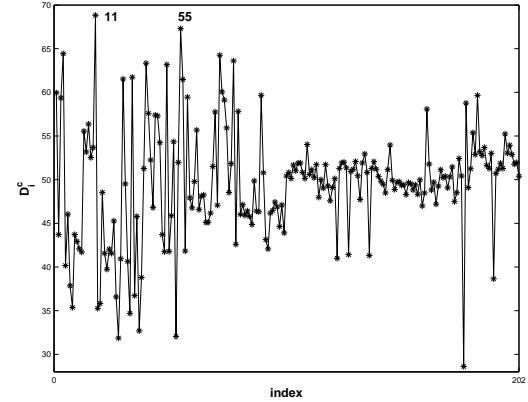

(a)

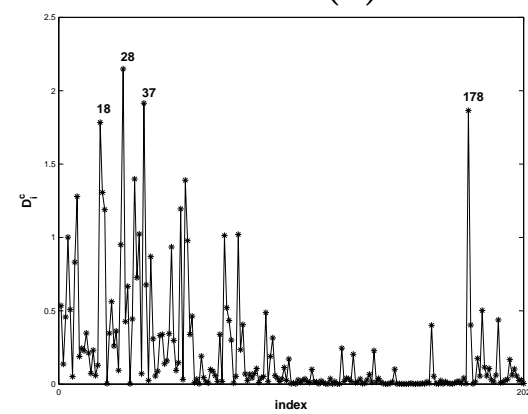

(c)

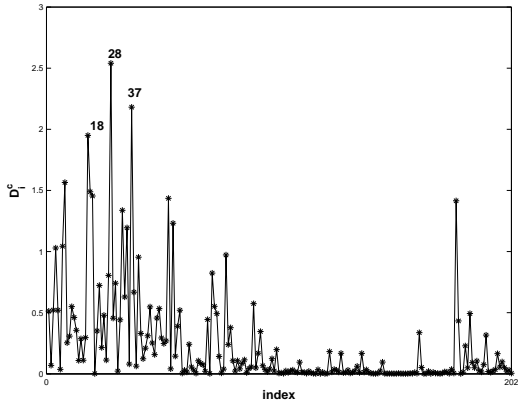

(b)

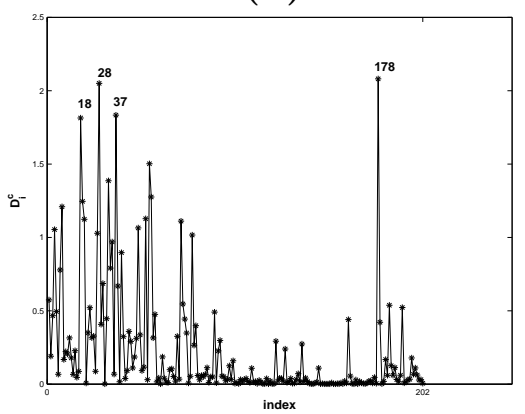

(d)

Figura C.8: Dados AIS. Influência global ( $\mathrm{Di}$ ) nos modelos assimétricos: (a) normal (b) t de Student (c) exponencial potência e (d) slash. 


\section{Referências}

1 Aigner, D., Lovell, C. e Schmidt, P. (1977). Formulation and estimation of stochastic frontier production function model. Journal of Econometrics, 12, 12-37.

2 Andrade, F.C. (2004). Pontos de alavanca em regressão. Dissertação de Mestrado, Departamento de Estatística, Universidade de São Paulo.

3 Aoki, R. (2001). Modelos de regressão com erros nas variáveis com intercepto nulo. Tese de doutorado. IME - Universidade de São Paulo.

4 Andrews, D.R. e Mallows, C.L. (1974). Scale mixtures of normal distributions. Journal of the Royal Statistical Society, B, 36, 99-102.

5 Arellano-Valle, R.B. e Azzalini, A. (2006). On the unification of families of skew-normal distributions. Scandinavian Journal of Statistics, 33 (3), 561-574.

6 Arellano-Valle, R.B., Bolfarine, H. e Lachos, V.H. (2005a). Skew-normal linear mixed models. Journal of Data Science, 3, 415-438.

7 Arellano-Valle, R.B., Bolfarine, H. e Lachos, V.H. (2007). Bayesian Inference for Skew-Normal Linear Mixed Models. Journal of Applied Statistics, 34, 6, 663-682(20).

8 Arellano-Valle, R.B. e Genton, M.G. (2005b). On fundamental skew distributions. Journal of Multivariate Analysis, 96, 93-116.

9 Arellano-Valle, R.B., Gómez, H.W. e Quintana, F.A. (2004). A new class of skew-normal distributions. Communications in Statistics, 33, 1465-1480.

10 Arellano-Valle, R.B., Gómez, H.W. e Quintana, F.A. (2005c). Statistical inference for a general class of asymmetric distributions. Journal of Statistical Planning and Inference, 128, $427-443$.

11 Arellano-Valle, R.B., Ozan, S., Bolfarine, H. e Lachos, V.H. (2005). Skew-normal measurement error models. Journal of Multivariate Analysis, 96, 265-281.

12 Arnold, B.C. e Beaver, R.J. (2000). The skew-Cauchy distribution. Statistics and Probability Letters, 49, 285-290.

13 Azzalini, A. (1985). A class of distributions which includes the normal ones. Scandinavian Journal Statistics, 12, 171-178. 
14 Azzalini, A. (1986). Further results on a class of distributions which includes the normal ones. Statistica, XLVI, 199-208.

15 Azzalini, A. (2005). The skew-normal distribution and related multivariate families(with discussion). Scandinavian Journal Statistics, 32, 159-188.

16 Azzalini, A., Capitanio, A. (1999). Statistical applications of the multivariate skew normal distribution. Journal of the Royal Statistical Society, ser. B, 61, 579-602.

17 Azzalini, A. e Capitanio, A. (2003). Distributions generated by perturbation of symmetry with emphasis on a multivariate skew t distribution. Journal of the Royal Statistical Society, ser. B, 65, 367-389.

18 Azzalini, A., Dal Capello, T. e Kotz, S. (2003). Log-skew-normal and log-skew-t distributions as model for family income data. Journal of Income Distribution, 11, 12-20.

19 Azzalini, A. e Dalla-Valle, A. (1996). The multivariate skew-normal distribution. Biometrika, 83, 715-726.

20 Banerjee, M. e Frees, E.W. (1997). Influence diagnostics for linear longitudinal models. Journal of the American Statistical Association, 92, 999-1005.

21 Basford, K.E., Greenway, D.R., McLachlan, G.J., e Peel, D. (1997). Standard errors of fitted means under normal mixture. Computational Statistics, 12, 1-17.

22 Bauwens, L. e Laurent, S. (2004). A new class of multivariate skew densities with application to GARCH models. Journal of Business and Economic Statistics, 23, 346-354.

23 Bazán, J.L.G. (2005). Uma família de modelos de resposta ao ítem normal assimétricas. Tese de doutorado. IME - Universidade de São Paulo.

24 Belsley, D.A. (1991). Conditioning diagnostics: collinearity and weak data in regression. Wiley, New York.

25 Billor, N. e Loynes, R.M. (1993). Local influence: a new approach. Communications in Statistics, Theory and Methods 22, 1595-1611.

26 Branco, M. e Dey, D. (2001). A general class of multivariate skew-elliptical distribution. Journal of Multivariate Analysis, 79, 93-113.

27 Capitanio, A., Azzalini, A., e Stanghellini, E. (2003). Graphical models for skew-normal variates. Scandinavian Journal of Statistics, 30, 129-144.

28 Chatterjee, S. e Hadi, A. S. (1988). Sensivity Analysis in Linear Regression. New York: John Wiley.

29 Conde, D.M., Pinto-Neto, A.M., Cabello, C., Santos-Sá, D., Costa-Paiva, C., e Martinez, E.Z. (2005). Quality of life in brazilian breast cancer survivors age 45-65 years: associated factors. The Breast Journal, 11, 425-432.

Ferreira, C. S. 
30 Cook, R.D. (1977). Detection of influential observation in linear regression. Technometrics, 19, 5-18.

31 Cook, R.D. (1986). Assessment of local influence (with discussion). Journal of the Royal Statistical Society, B, 48, 133-169.

32 Cook, R,D. e Weisberg, S. (1982). Residuals and Influence in Regression. London: Chapman and Hall.

33 Cox, D.R. e Hinkley, D.U. (1974). Theoretical Statistics. London: Chapman and Hall.

34 Christensen, R., Pearson, L.M. e Johnson, W. (1992). Case-deletion diagnostics for mixed models. Technometrics, 34, 38-45.

35 Dempster, A.P., Laird, N.M. e Rubin, D.B. (1977). Maximum likelihood from incomplete data via the EM algorithm. Journal of the Royal Statistical Society, B, 39, 1-22.

36 Dempster, A.P., Laird, N.M. e Rubin, D. B. (1980). Iteratively reweighted least squares for linear regression when errors are Normal/Independent distributed. Em P. R. Krishnaiah (Ed.), Multivariate Analysis V, 35-57. North-Holland.

37 Diggle, P.J., Liang, K.Y. e Zeger, S.L. (1994). Analysis of Longitudinal Data. Oxford: Clarendon Press.

38 Emerson, J.D., Hoaglin, D.C. e Kempthorne, P.J. (1984). Leverage in least squares additive-plus-multiplicative fits for two-way tables. Journal of the Royal Statistical Society, 79, 329-335.

39 Fang, K.T., Kotz, S., Ng, K.W. (1990). Symmetric Multivariate and Related Distributions. New York: Chapman and Hall.

40 Fang, K.T. e Zhang, Y.T. (1990). Generalized Multivariate Analysis. New York: Springer-Verlag.

41 Farrell, P. J. e Cadigan, N. G. (2000) Local influence in binary regression models and its correspondence with global influence. Communications in Statistics-Theory and Methods, 29, 349-368.

42 Fernández, C. e Steel, M.F.J. (1999). Multivariate Student-t regression models: Pitfalls and inference. Biometrika, 86, 153-167.

43 Fei, Y. e J. Pan, (2003). Influence assessments for longitudinal data in linear mixed models. Em G. Verbeke; G. Molenberghs; M. Aerts e S. Fieuws (Eds.), Statistical Modelling: Proceedings of the 18th Interna- tional Workshop on Statistical Modelling, 143-148. Leuven, Bélgica.

44 Firth, D. (1993). Bias reduction of maximum likelihood estimates, Biometrika, 82, $27-38$. 
45 Freitas, L.A. (2005). Modelo de regressão com erros normais assimétricos: uma abordagem bayesiana. Dissertação de Mestrado, Departamento de Estatística, Universidade Federal de São Carlos. São Carlos.

46 Galea, M. (1995). Calibração comparativa estrutural e funcional. Tese de Doutorado, Departamento de Estatística, IME-USP. São Paulo.

47 Galea-Rojas, M., de Castillho, M., Bolfarine, H. e de Castro, M. (2003). Detection of analytical bias. Analyst, 128, 1073-1081.

48 Galea-Rojas, M., Paula, G.A. e Bolfarine, H. (1997). Local influence in elliptical linear regression models. The Statistician, 46, 71-79.

49 Galea-Rojas, M., Paula, G.A. e Uribe-Opazo, M. (2003). Influence diagnostics in univariate linear elliptical regression model. Statistical Paper, 44, 23-45.

50 Genton, M.G., ed. (2004). Skew-elliptical distributions and their applications: a journey beyond normality. London: Chapman and Hall.

51 Genton, M.G., He, L., Liu, X. (2001). Moments of skew-normal random vectors and their quadratic forms. Statistics and Probability Letters, 51, 319-325.

52 Genton, M.G. e Loperfido, N. (2005). Generalized skew-elliptical distributions and their quadratic forms. Annals of the Institute of Statistical Mathematics, 57, 389-401.

53 Gómez, H.W. (2005). Extensiones Asimétricas de Distribuciones Simétricas: Propiedades e Inferência. Tese de Doutorado. Facultad de Matemáticas, Pontifícia Universidad Católica de Chile. Santiago, Chile.

54 Gómez, H.W., Venegas, O. e Bolfarine, H. (2007). Skew-symmetric distributions generated by the normal distribution function. Environmetrics, 18, 395-407.

55 Gupta A.K. (2003). Multivariate skew t distributions. Statistics, 37, 359-363.

56 Gupta, R.C. e Gupta, R.D. (2004). Generalized Skew Normal Model. Sociedad de Estadística e Investigación Operativa Teste, 13, 501-524.

57 Gupta, A.K. e Huang, W.J. (2002). Quadratic forms in skew normal variates. Journal of Mathematical Analysis and Applications, 273, 558-564.

58 Henderson, C.R. (1984). Applications of linear models in animal breeding. University of Guelph Press, Guelph.

59 Henze, N. (1986). A probabilistic representation of the "skew-normal" distribution. Scandinavian Journal of Statistics, 13, 271-275.

60 Hill, M.A. e Dixon, W.J. (1982). Robustness in real life: a study of clinical laboratory data. Biometrics, 38, 377-396. 
61 Hoaglin, D.C. e Welsh, R.E. (1978). The hat matrix in regression and ANOVA. The American Statistician, 32, 17-22.

62 Johnson, N.L., Kotz, S., e Balakrishnan, N. (1994). Continuous Univariate Distributions, vol. 1. New York: John Wiley.

63 Jones, M.C. e Faddy, M.J. (2003). A skew extension of the $t$ distribution, with applications. Journal of the Royal Statistical Society, B, 65, 159-174

64 Lachos, V.H. (2004). Modelos lineares mistos assimétricos. Tese de Doutorado, Departamento de Estatística, IME-USP. São Paulo.

65 Lachos, V.H. e Bolfarine, H. (2007). Skew probit error-in-variables models. Statistical Methodology, 3, 1-12.

66 Lachos, V.H., Bolfarine, H., Arellano-Valle, R.B. and Montenegro, L.C. (2007a). Likelihood based inference for multivariate skew-normal regression models. Communications in Statistics: Theory and Methods, 36, 1769-1786.

67 Lachos, V.H., Bolfarine H. e Montenegro, L.C. (2007b). Inference and assessment of local influence in skew-normal null intercept measurement error models. Journal of Statistical Computation and Simulation, 78, 395-419.

68 Lachos, V.H., Vilca, L.F. e Galea, M. (2006). Influence diagnostics for Grubbs's model. Statistical Papers, 48, 419-436.

69 Laird, N.M. e Ware, J.H. (1982). Random effects models for longitudinal data. Biometrics, 38, 963-974.

70 Lange, K. L., Little, J.A. e Taylor, M.G.J. (1989). Robust modeling using the $t$ distribution . Journal of the American Statistical Association, 84, 881-896.

71 Lange, K. e Sinsheimer, J.S. (1993). Normal/independent distributions and their applications in robust regression. Journal of Computational and Graphical Statistics, 2, 175-198.

72 Lee, Y. e Nelder, J.A. (2004). Conditional and marginal model: another view. Statistical Science, 19, 219-238.

73 Lee, S. e Xu, L. (2004). Influence analysis of nonlinear mixed-effects models. Computational Statistics and Data Analysis, 45, 321-341.

74 Lesaffre, E. e Verbeke, G. (1998). Local influence in linear mixed models. Biometrics, $\mathbf{5 4}, 570-582$.

75 Lin, T.I. e Lee, J.C. (2008). Estimation and prediction in linear mixed models with skew-normal random effects for longitudinal data. Statistics in Medicine, 27, 1490-507. 
76 Lin, T.I., Lee, J.C. e Yen, S.Y. (2007). Finite mixture modeling using the skew normal distribution. Statistica Sinica, 17, 909-927.

77 Liseo, B. e Loperfido, N. (2003). A bayesian interpretation of the multivariate skew-normal distribution. Statistics and Probability and Letters, 61, 395-401.

78 Liseo, B. e Loperfido, N. (2006). A note on reference priors for the scalar skew-normal distribution. Journal of Statistical Planning and Inference, 136, 373-389.

79 Little, R.J.A. (1988). Robust estimation of the mean and covariance matrix from data with missing values. Applied Statistics, 37, 23-38.

80 Liu, S.Z. (2000). On local influence for elliptical linear models. Statistical Papers, 41, 211-224.

81 Louis, T.A. (1982). Finding the observed information matrix when using the EM algorithm. Journal of the Royal Statistical Society, B, 44, 226-233.

82 Lu, B. e Song, X.Y. (2006). Local influence of multivariate probit latent variable models. Journal of Multivariate Analysis, 97, 1783-1798.

83 MATLAB for Windows User's Guide, The Math Works Inc., 1991.

84 Nadarajah, S. e Kotz, S. (2003). Skewed distributions generated by the normal kernel. Statistics Probability Letters, 65, 269-277.

85 Nobre, J.S. e Singer, J.M. (2006). Fixed and random effects leverage for influence analysis in linear mixed models. Submetido.

86 O'Hagan, A. e Leonard, T. (1976). Bayes estimation subject to uncertainty about parameter constraints. Biometrika 63, 201-202.

87 Pace, L. e Salvan, A. (1997). Principles of Statistical Inference. Singapore: World Scientific.

88 Pewsey, A. (2000). Problems of inference for Azzalini's skew-normal distribution. Journal of Applied Statistics, 27, 859-870.

89 Poon, W.Y. e Poon, Y.S. (1999). Conformal normal curvature e assessment of local influence. Journal of the Royal Statistical Society, B, 61, 51-61.

90 R Development Core Team, (2005). R: A language and environment for statistical computing. R Foundation for Statistical Computing, Vi-enna, Austria. URL http://www.Rproject.org.

91 Roberts, C. (1966). A correlation model useful in the study of twins. Journal of the American Statistical Society, 61, 1184-1190.

92 Rodríguez, C.L.B. (2005). Inferência bayesiana no modelo normal assimétrico. Dissertação de mestrado. Departamento de Estatística, IME-USP. São Paulo.

Ferreira, C. S. 
93 Ross, W.H. (1987). The geometry of case delection and the assessment of influence in nonlinear regression. The Canadian Journal of Statistics, 15, 91-103.

94 Sahu, S.K., Dey, D.K. e Branco, M. (2003). A new class of multivariate skew distributions with applications to bayesian regression models. The Canadian Journal of Statistics, 31, 129-150.

95 Salgado, F.A.O. (2006). Diagnóstico de influência em modelos elípticos com efeitos mistos. Tese de Doutorado, Departamento de Estatística, IME-USP. São Paulo.

96 Sartori, N. (2003). Bias prevection of maximun likelihood estimates: skew normal and skew $t$ distribuitions. Technical report. Dip. Scienze Statistiche, Università di Padova, Italy.

97 St. Laurent, R.T. e Cook, R.D. (1992). Leverage and superleverage in nonlinear regression. Journal of the American Statistical Association, 87, 985-990.

98 Tan, F. e Peng, H. (2006). The slash and the skew-slash Student t distributions. Submitted to Elsevier Science.

99 Vilca-Labra, F. e Leiva-Sánchez, V. (2006). A new fatigue life model based on the family of skew-elliptical distributions. Communications in Statistics - Theory and Methods, 35, 229-244.

100 Walker, S. (1996). An EM algorithm for nonlinear random effects models. Biometrics, 52, 934-944.

Wang, J., Boyer, J. e Genton, M.G. (2004). A skew-symmetric representation of multivariate distributions. Statistica Sinica, 14, 1259-1270.

101 Wang, J. e Genton, M. (2006). The multivariate skew-slash distribution. Journal of Statistical Planning and Inference, 136, 209-220.

102 Wei, B.C., Qu, Y.Q. e Fung, W.K. (1998). Generalized leverage and its applications. Scandinavian Journal of Statistics, 25, 25-37.

103 Wolfinger, R., Tobias, R. e Sall, J. (1991). "Mixed Models: A Future Direction". SUGI, 16, 1380-1388.

104 Wu, X. e Luo, Z. (1993). Second-order approach to local influence. Journal of the Royal Statistical Society, B, 55, 929-936.

105 Zacks, S. (1971). The Theory of Statistical Inference. New York: John Wiley.

106 Zhou, T. e He, X. (2007). Three-step estimation in linear mixed models with skew-t distributions. Journal of Statistical Planning and Inference. In Press.

107 Zhu, H. e Lee, S. (2001). Local influence for incomplete-data models. Journal of the Royal Statistical Society, B, 63, 111-126. 
108 Zhu, H. e Lee, S. (2003). Local influence for generalized linear mixed models. The Canadian Journal of Statistics, 31, 293-309.

109 Zhu, H., Lee, S., Wei, B.C. e Zhou, J. (2001). Case-deletion measures for models with incomplete data. Biometrika, 88, 727-737.

110 Zhu, H. e Zhang, H. (2004). A diagnostic procedure based on local influence. Biometrika, 91, 579-589.

Ferreira, C. S. 